\title{
Report to the American Physical Society by the study group on research planning for coal utilization and synthetic fuel production
}

\author{
APS Study Group Participants
}

Bernard R. Cooper, Chairman

West Virginia University, Morgantown, West Virginia 26506

Wayne R. Gruner, Executive Director

6305 Tulsa Lane, Bethesda, Maryland 20034

Larry Anderson,

University of Utah, Salt Lake City, Utah 84112

Robert H. Davis

Florida State University, Tallahassee, Florida 32306

Paul Engelking

University of Oregon, Eugene, Oregon 97403

Jose D. Garcia

University of Arizona, Tucson, Arizona 85721

Edward Gerjuoy

University of Pittsburgh, Pittsburgh, Pennsylvania 15260

Robert I. Jaffee

Electric Power Research Institute, Palo Alto, California 94304

Philip G. Kosky

General Electric Research and Development Center, Schenectady, New York 12301

Leonidas Petrakis

Gulf Research and Development Company, Pittsburgh, Pennsylvania 15230

Robert T. Poe

University of California, Riverside, California 92521

Richard Pollina

Montana State University, Bozeman, Montana 59717 


\section{Dwight Prater}

Mobil Research and Development Center, Paulsboro, New Jersey 08066

Robert L. Thomas

Wayne State University, Detroit, Michigan 48202

\section{Sandor Trajmar}

California Institute of Technology, Jet Propulsion Laboratory, Pasadena, California 91103

\section{Ex Officio Panel Members}

\section{G. J. Dienes}

Brookhaven National Laboratory, Upton, New York 11973

Nancy M. O'Fallon

Argonne National Laboratory, Argonne, Illinois 60439

\section{APS Council Review Committee}

Harvey Brooks, Chairman'.

Harvard University, Cambridge, Massachusetts 02138

\section{William A. Fowler}

California Institute of Technology, Pasadena, California 91125

\section{Everett Gorin}

72 Longwood Drive, San Rafael, California 94901

\section{Norman F. Ramsey}

Harvard University, Cambridge, Massachusetts 02138

\section{D. Schlesinger \\ 4766 Wallingford Street, Pittsburgh, Pennsylvania 15213}

The report presented here is the result of a study conducted over a period of approximately fourteen months, commencing in April 1980, on research planning for coal utilization and synthetic fuel production. This study is the most recent one of a series on topics related to energy technologies, initiated and organized by the American Physical Society through its Panel on Public Affairs (POPA). Financial support for the work of the study panel has been provided by the U.S. Department of Energy, through the Office of Energy Research and the Office of the Assistant Secretary for Fossil Energy. The report is directed toward a twofold audience. The primary intended audience is that of physicists, particularly those who may become active in the research areas discussed here. Our aim is to show these physicists where useful research can be done, to show the fundamental scientific interest in the particular work, and to give enough information to get people started. Our second intended audience is the DOE sponsors and others in the "user community". Here we aim to suggest areas where contributions from physicists may most profitably be sought and to suggest what resources are necessary to optimize that contribution. 


\section{CONTENTS}

I. Summary and Principal Recommendations

II. Introduction

III. Research on Coal

A. Primer on coal

1. Origin and petrography

2. Classification and nomenclature

3. Chemical structure

4. Physical structure

5. References

B. Pore structure of coal

1. Introduction

2. Research areas

a. Quantitative characterization of coal pore size distribution

b. Study of molecular species resident in and moving through pore structure

3. Recommendations

4. References

C. Analytical techniques

1. Introduction

2. Selected problem areas

a. Elemental composition

b. Chemical structure

c. Physical structure

d. Surface characterization

e. Physical and chemical transformations

3. Selected techniques

a. Introduction

b. Spin resonance

c. Electromagnetic spectroscopies

d. Electron probe and electron spectroscopy

e. Other techniques

4. Recommendations

5. Summary

6. References

IV. Research for the Technologies

A. Primer on coal utilization technologies

1. Pyrolysis

2. Synthetic fuels

3. Gasification: Importance of general features

4. Flow regimes in heterogenous reactors

5. Some specific gasifiers

6. Liquefaction

a. Some important direct processes

b. Indirect processes

7. Direct combustion

a. Pulverized coal combustion in boilers

b. Fluidized bed combustion

8. Advanced concepts embodying combined cycles

a. Pressurized fluidized bed combined cycle b. Magneto-hydrodynamic conversion "MHD"

c. High temperature fuel cells

9. Mining

10. Coal preparation

11. References

B. Instrumentation and control

1. Introduction

a. General nature of the difficulty

b. Plan of presentation

2. Instrumentation and control needs and problems

a. Level measurement

b. In-situ analysis of solid and/or liquid process streams

c. Gas analysis

d. Pressure

3. Multiphase mass flow measurement

4. Monitoring of particulates

a. General discussion

b. Intensity ratio of forward scattered laser light

c. Visibility method using split laser beams

d. Particle sizing using laser diffraction

e. Photon correlation spectrometry

f. Impactors

g. Overview

5. Temperature measurements

a. Thermometry for coal technology

b. Temperature measurements and modeling

6. Potentialities of acoustics

a. Reasons for investigating acoustic techniques

b. Present applications of acoustics in coal utilization

c. Some possible applications of acoustics to coal technology

7. Recommendations-instrumentation and control

8. References

C. Modeling and flow theory

1. Introduction

2. Modeling at the particulate level

3. Zone models

4. Example of modeling at the coal reactor level

5. Multiphase flow modeling

6. Need for model test facility

7. Theoretical aspects: Summary and recommendations

8. References

D. Materials in coal conversion and utilization

1. Introduction

2. Environmental fatigue and fracture

a. The hydrogen environment

b. Crack nucleation and growth 
3. Erosion

4. Corrosion

5. Structural ceramics

6. Refractories and slags
a. Refractories

b. Slags

7. Recommendations

8. References

E. Size dependent phenomena

1. Introduction

2. Coal preparation

3. Mechanical properties of coal

4. Chemical comminution

5. Coal-oil mixtures

6. Coal-water systems

7. Beneficiation/high gradient magnetic separation (HGMS)

8. Recommendations
9. References

F. Catalysis

1. Introduction

2. Zeolite size and shape selective catalysts

3. Mineral matter catalytic effects in direct liquefaction

4. Surface science related to transition metal heterogeneous catalysts for gas synthesis

5. Catalyzed gasification of carbons and coals

6. Denitrogenation and desulfurization catalysts

7. Recommendations

8. References

Appendix I. $\mathrm{CO}_{2}$ Emissions: Comparisons of the Use of Coal for Energy Production by Various Routes

Appendix II. Acknowledgments

Appendix III. Glossary 


\section{SUMMARY AND PRINCIPAL RECOMMENDATIONS}

This report is meant to provide guidance to scientists and planners for maximizing the contribution of physics research to improvement of coal technology, including synthetic fuel production. The Study Panel has identified a set of research issues having potential usefulness for the development of the technology, as well as offering opportunities for increasing our understanding of fundamental physical processes.

The areas selected include both research on coal itself and research pertinent to technology development. To gain improved understanding of coal as a material we have focused on the development of new and improved analytical techniques, and we also offer suggestions for work to clarify the nature and role of the complex pore networks characteristic of the physical structure of coals. The research issues pertinent to technology development include instrumentation and control, modeling and flow theory, materials problems, size-dependent phenomena (size reduction of coal and coal/fluid interactions), and cataysis. Research assessments have been developed for these selected problems, and recommendations offered. For success in attaining the desired progress in these areas, and in coal utilization and synthetic fuel technology generally, cooperative efforts of physicists working with other physical-phenomena-oriented researchers (chemists, materials scientists, engineers) are necessary. It is highly desirable to consciously foster such and interdisciplinary effort. One contribution toward this is to organize interdisciplinary symposia and topical conferences in research pertinent to coal utilization and synthetic fuel production, as is being done under the auspices of the American Physical Society Committee on the Applications of Physics.

Environmentally acceptable utilization of coal as an energy source is a central motivation for the technology, and hence the pertinent research, for coal utilization and synthetic fuel production. Many of the research areas we discuss affect monitoring and controlling production of undesirable substances. We have not dealt with questions of environmental impact as such. However, the coupling between success in the research that is the topic of this report and the minimization of adverse environmental effects, should be borne in mind. Since $\mathrm{CO}_{2}$ is an inevitable emission in the utilization of fossil fuels, and the amount produced is inversely related to the thermal efficiency of processes, we have deemed it appropriate and useful to provide an analysis comparing various coal utilization schemes as sources of $\mathrm{CO}_{2} \cdot$

If our research recommendations bear fruit, that should help to overcome some key obstacles in the way of improved coal utilization and synthetic fuel production technology. Here we present our highest priority recommendations, both research and institutional. More detailed lists of research recommendations appear in the sections treating each selected research area.

\section{A. Principal research recommendations (not ranked)}

- We recommend that support for physics research in the area of coal utilization make provision for a non-negligible percentage of highly novel, even speculative, approaches to outstanding applied problems. The potential economic payoffs from solutions to these problems is so great that support for unusual nontraditional approaches is much more justified in the coal research area than in most applied areas.

- We recommend that presently available and developing techniques be applied to the determination of chemical structural features of coals, including hydroaromatic and aromatic cluster configurations, functional groups, and molecular weight distributions. Good candidates for such techniques are magnetic resonance, vibrational, laser, photoelectron, mass, and X-ray spectroscopies.

- We recommend systematic analyses of the physical properties of coals to elucidate the pore structure of coal particles including pore shapes and pore size distributions. There should be emphasis on measurements under dynamic conditions, i.e. following changes with time under realistic operating conditions of temperature and pressure. This could be done by use of small angle $X$-ray and neutron scattering, X-ray tomography, and electron microscopy.

- We recommend strenuous efforts to develop better dynamic models of advanced coal processes. Such models are necessary to 
permit reliable scale-up of the processes and to serve as the bases for design of control systems. Economic motivations and considerations of safety provide considerable incentive for this research. Very short response times or the need to integrate subsystems with very different time constants often compound the difficulties.

- We recommend initiation of a carefully planned program to evaluate presently available instruments, and where necessary to develop new instruments, usable both for systematic collection of data on advanced coal processes and for incorporation into control systems for these processes. Such data acquisition is necessary for producing and verifying dynamic models as recommended above. Instruments are needed which can measure multiphase flow, temperature, particulate matter, compositions (without sampling) of dense phase and gaseous material, levels of gas-solid and liquid-solid interfaces, pressure, and viscosity of the process materials in plants. The measurements must often be done in real time under adverse conditions. Attention should be given to novel and advanced (e.g. acoustic and laser) techniques, which have been insufficiently exploited in the past.

- We recommend an intensive effort on theoretical work needed to formulate problems concerned with multiphase flow behavior, and to develop innovative computer simulations of such behavior. Such research is important to further progress in coal utilization, and can lead to improvements in efficiency and significant decreases in undesirable emissions.

- We recommend an extensive research effort to understand the effects of coal conversion and combustion environments on materials of construction (alloys, refractory ceramics, composites, etc.), including long duration exposure to the process environment. The ability to predict such effects is essential to ensure reliable and economic operation under process conditions which may reach temperatures as high as 2600C (MHD combustor) and pressures to $2000 \mathrm{psi}$, which involve atmospheres that are either highly oxidizing or reducing (or that alternate cyclically between these), and which may include soaking in a highly corrosive slagging environment, or exposure to erosive, particle-laden stream flows (as in the direct coal-fired gas turbine, for example). Hopefully, such understanding will also lead to the improvement of pertinent materials properties. The recommended research effort should involve: a microscopic approach, using the techniques of surface science and theoretical semi-empirical potential modeling, to elucidate the effect of the environment (especially hydrogen) on fatigue and fracture phenomena, a fundamental analysis of the physical mechanisms in solid particle erosion processes; and a study of mass and charge transfer through solid and liquid oxides.

- We recommend pursuing experimental and theoretical investigations to delineate the phenomena in size reduction processes, such as grinding and chemical comminution. Current practices and technologies are inefficient; and they involve large expenditures of energy, especially to make very small particles. Efforts need to be made to relate the mechanical properties and physical (pore) structure to fracture initiation and propagation in coals.

- We recommend further work to bring sophisticated spectroscopic methods of solid state and surface physics to bear on systems with compositions, and conditions of pressure and temperature, as closely akin as possible to those used in practical heterogeneous metal catalysis for fuel synthesis, i.e., starting from syngas ( $\mathrm{H}_{2}$ and $\mathrm{CO}$ ) mixtures obtained from coal gasification. It is particularly desirable to develop spectroscopic technique to study intermediate compounds formed on metal surfaces in catalytic synthesis and in reacting coal chars.

- We recommend increased emphasis on understanding and exploiting the properties of size and shape selective catalysts. There are attractive opportunities for fundamental science with great potential impact on the development of indirect liquefaction technology. It is of particular interest to understand diffusion processes in zeolite systems of this type, and to make and study systems with very small particles of transition metals imbedded in zeolites.

\section{B. Principal institutional recommendations}

- We recommend the establishment of a sample bank of well selected, characterized, and preserved coal samples, available to research and analytical groups. This should be preceded by research sufficient to verify the chosen storage technique(s). 
This recommendation makes explicit our strong agreement with the primary recommendation of the recent Basic Coal Science Project Advisory Committee Report to the Gas Research Institute (GRI), and our wish to encourage the existing interest in the U.S. Department of Energy (DOE) in establishment of such a sample bank. A recent workshop (March 27-28, 1981), sponsored by GRI and DOE, was held to delineate the current sample needs of the research and technology communities. The establishment of a National Premium Coal Sample Bank is under consideration as a result of this workshop and other meetings of interested groups. Such establishment would meet the aims of our recommendation, provided that the quality and availability of such samples is maintained for analytical and research purposes.

For the maximum effectiveness of the research and analyses recommended in our present report to be achieved, standard materials need to be available to many research workers. In the past, the effectiveness and reproducibility of research in coal science has been adversely affected by insufficient attention to sample quality. The complex heterogeneous nature of coal from any one source, together with the diversity of coals from different sources, makes careful attention to sample selection and characterization essential if different researchers' results are to be compared and synthesized into a larger understanding. We recognize that there is no consensus at this time as to the preferred route of stable storage of coal samples. Our coal sample bank recommendation therefore includes doing sufficient research to verify the validity of the chosen storage technique(s).

The implementation of this recommendation for a National Premium Coal Sample Bank should be in stages. The initial stage should be to use the best of the currently available techniques, e.g. coal storage in deionized water at a few degrees Celsius, coal storage in deionized ice, or coal storage in an inert atmosphere of nitrogen or argon. Each of these techniques has certain disadvantages in not preserving one or more moieties in the coal. Therefore our recommendation includes a continuing longer term program of coal characterization, including effects of storage, to further refine the storage modes and to define new methods of storage for the samples in the Premium Coal Bank. Consideration should also be given to storage of samples from different locations in a seam and in different states of aggregation, varying from sizeable pieces to ground samples. We recognize that in addition to the recommended storage bank for premium samples, to be available in relatively limited quantities, the need will continue for other, already existing, sample banks that have larger samples available for purposes requiring less rigorous storage control.

- We recommend establishment of a program to gain fundamental understanding of processes for coal utilization and synthetic fuel production. This would focus on the development and testing of theoretical models and of process control techniques, together with the development of instrumentation for acquisition of the data necessary for this purpose.

We recommend that a significant program aimed at fundamental understanding and improvement of coal utilization and synthetic fuel production processes be undertaken, with the focus on modeling of liquefaction and gasification reactors, combustors, and multiphase flow systems. This program would include development of instrumental techniques for use at dedicated test facilities to collect data necessary for development and verification of models, and might have to involve construction of new facilities specially designed to achieve the objectives of this program. The increased understanding of the process kinetics will permit reliable scale-up, and will aid the design of better processes. Benefits will also accrue for the development of control strategies and instrumentation.

In order to: encourage optimum use of people and test facilities at a variety of institutions (universities, government and non-profit laboratories, industry); systematize the data collection and retrieval; and plan for the best use of funds for expanded or additional facilities, -- it is recommended that overall responsibility for long range planning, coordination and technical oversight be assigned to an appropriate group. This group could be at a national laboratory, university, or non-profit organization, or it might consist of a committee made up of representatives from such institutions, as well as from industry. The activities of the Gas Research Institute and the Electric Power Research Institute serve as models, suggesting an arrangement in which the research work would be distributed to existing 
laboratories.

In the area of instrumentation development, the program would not aim to carry instrumentation all the way to commercialization, but rather to advance the state of the art in key instrumentation areas, both by coordinating research and by providing a central repository of information on diagnostic and process control instrumentation. The ideal would be a cooperatively funded program, with long range funding coming partly from government agencies and partly from industry.

In planning and establishing the suggested program, the relationship of instrumentation for data acquisition to the development and validation of process models is central. The refinement of such models has economic value through the resulting refinement of coal utilization technologies. Under current practice, instrumentation efforts are devoted almost entirely to operation and control of existing working systems, while insufficient effort has been devoted to systematic collection of data to test reliability of modeling concepts, i.e. to provide the data necessary to model a priori for better processes. Setting up the proposed program should help to correct this deficiency.

Rev. Mod. Phys., Vol. 53, No. 4, Part II, October 1981 


\section{INTRODUCTION}

All plans giving the U.S.A.'s energy needs and resources for the transition decades from the twentieth into the twenty-first century project a substantially increasing utilization of coal. It is therefore important to deal with the technology problems of that increased use, including the trade-offs of time and cost, particularly bearing in mind that the use of coal must be done in an environmentally acceptable manner. There is an important and interesting role for physicists to play in these problems. The report presented here is the result of a study conducted over a period of approximately fourteen months, commencing in April 1980, on research planning for coal utilization and synthetic fuel production. Financial support for the work of the study panel has been provided by the U.S. Department of Energy, through the Office of Energy Research (OER) and the Office of the Assistant Secretary for Fossil Energy (ASFE). Our aim is to provide guidance to scientists and planners in maximizing the positive impact of current and future physics research on the development of technology for coal energy utilization. By example, we will show that for physicists the questions arising out of the needs of coal technology lead to science that is interesting and significant in itself.

A synthetic fuel production program based on coal requires an investment of hundreds of billions of dollars and a probable time period of decades to meet a relatively modest $(10-15 \%)$ fraction of the country's liquid fuel needs. In addition, advanced technologies using coal for the generation of electricity call for further heavy investment of time and money. The latter level of investment is significant, in part, because more than incremental technology and retro-fit is envisaged. For example, the coal-fired integrated gasification combined cycle (i.e., gas and steam turbines) power plant may supersede the conventional coal power plant, since the former has comparable or higher efficiency and superior emissions ( $\mathrm{NO}_{X}, \mathrm{SO}_{X}$ and particulates) control. This development holds the promise of relieving the present use of oil and natural gas without the level of environmental damage normally attributed to coal.

Because of the long lead time to put the new synthetic fuel and electricity generating technologies into place, research must be done now for impact on the energy systems of ten or twenty years, or even longer, from now.* Also, information gained from research can crucially affect planning for the very large investments involved in development and demonstration, as well as the ultimate vast capital investment involved in production facilities. The aggregate investment required is such that even a small percentage reduction in costs resulting from research findings could have dramatic economic impact. Much of the research discussed in Part IV, Research for the Technologies, is also pertinent to the production of liquid fuels from oil shale.

Our report is oriented primarily toward physicists. A number of physicists already are active in coal related research, but that number is small relative to the needs and challenges of the problems. Problems such as those of materials and instrumentation, very much in the bailiwick of physicists, are central to the economic viability of coal technologies. Theoretical modeling of the huge reactors necessary for coal conversion to gaseous and liquid fuels is basically an engineer's problem, but a large element in that problem, the linkage between modeling and instrumentation, has been neglected. The physicist has unique qualification to critically examine and improve the physical assumptions of a model, and to devise instrumentation for acquiring data to test that model.

Understanding and control of multiphase flow is a central issue throughout the technology. Historically, fundamental understanding of this question is a physicist's game, and this seems to be the time for physicists to reactivate this interest. There is a need in the multiphase flow theory area for a serious look to see whether advances in computing technique in recent years offer opportunity to include enough of the essential physics to gain real understanding. Also, further data are necessary on which to base any such computational advances.

*For general discussion of research needs for coal utilization and synthetic fuel production see Energy Research Advisory Board, 1980; Fossil Energy Research Working Group, 1979 and 1980; Gas Research Institute, 1980; Gorbaty et al., 1979; Grimes et al., 1980; Keller, 1979; National Academy of Sciences, 1979. 
In addition to working on problems affecting materials of construction, many surface, chemical, and solid-state physicists can expect to beneficially affect catalysis questions central to the economic performance of the technologies. Physicists have played a significant role in gaining understanding of the crystal structures of the size and shape selective zeolite catalysts that are coming to dominate refining technology. Yet knowledge of these materials, and the opportunities and needs for new research on them for synthetic fuel technology, is not widespread among physicists. In fact, opportunities offered by the striking nature of zeolite crystal

structures is a beautiful example of the genuinely new science that relates to the needs of coal technology. Zeolite structures contain crystallographically defined cavities that are perfectly designed to hold tiny particles of transition metals (, $7-10 \mathrm{~A}$ in diameter). Moreover, needs of practical catalysis chemistry have provided technique for making samples with such embedded metal particles. Such systems offer exciting prospects for exploration ôf size and surface-dependent electronic properties; and one has the happy situation, described more fully in Section IVF, that these systems offer the possibility of combining desired features from two competing methods for the synthesis of high octane gasoline from syngas ( $\mathrm{CO}$ and $\mathrm{H}_{2}$ mixture) produced by coal gasification.

The objective of much of the technology we will discuss is conceptually simple. Coal has major deficiencies as a fuel. These affect environmental acceptability and convenience in use. Coal is non-uniform; it contains impurities; and its solid form is inconvenient for many uses, especially as a transportation fuel. These problems can be solved by liquefaction or gasification, both of which in essence simply involve increasing the ratio of hydrogen to carbon, i.e., the H/C ratio in coal is much lower than in petroleum and lower yet than that in natural gas (methane). Of course, this addition of hydrogen is not simple. A great complication is that the starting material, coal, is complex and poorly understood. Thus development of analytical techniques is central to the hope for genuinely new technology. Traditionally, physicists have played a leading role in the development of new analytical techniques. Often these techniques have then been refined and applied by other workers. A good example in the coal area is the introduction of Mossbauer spectroscopy as an analytical tool. Use of this technique to characterize coal impurities (some of which have an important catalytic function) and transformations during processing was introduced by physicists only a few years ago. Yet already Mossbauer spectroscopy has become a standard technique.

To design efficient ways for reacting coal, we require knowledge of its complex physical structure, as well as of the chemical structure. This physical structure basically consists of an intricate mesh, with pores of varying size. The pore structure controls the access and egress of reagents and reaction products. Development of nonintrusive physical probes of this structure, such as nuclear magnetic resonance (NMR) and small angle $x$-ray and neutron scattering (SAXS and SANS) are desirable to delineate the nature of the pore structure and the processes by which reactive species migrate through it. A particularly promising way for physicists to contribute to the necessary understanding is by designing research programs which coordinate the measurements of molecular migration on different time scales, e.g. recent studies of water in coal have successfully correlated results from specific heat and NMR measurements.

Besides being addressed to the physics community, our report is intended to provide the DOE sponsors, and others in the "user community", with some indication of how a group - - predominantly physicists, but containing chemists and chemical engineers -representing quite a wide range of specializations, perceives the scientific aspects of coal utilization after a conscientious reconnaissance -- and to suggest areas where contributions from physicists may be most profitably sought, as well as to suggest what resources are necessary to optimize that contribution.

\section{Nature of the study}

The study reported here is the most recent one of a series initiated and organized by the American Physical Society through its Panel on Public Affairs (POPA). At present, physicists have participated only to a limited extent in coal utilization related research. Besides input on specific technical questions, it was desired in general to gain the new point of view resulting from an increasing 
participation by physicists in this research area. As part of gaining a "fresh look", roughly half of the study panel members had little or no previous experience in coal. The other half of the panel, in quite varying degrees, were already familiar with the area of coal science and technology, and provided the experience base necessary to prevent "reinventing the wheel." In addition to the knowledge brought in by these people, the study panel sought and studied the background knowledge and views of a great number of consultants. These consultants are listed in Appendix II. In composing the panel, a diversity of background and interest played a strong role. While physicists are in the majority, the panel also contains chemists and chemical engineers.

At the outset it was decided to aim at selectivity -- to identify and treat a limited number of important technical issues in some depth, rather than to aim at a comprehensive treatment. After an extended period of information gathering and discussion, the panel selected seven priority areas for detailed assessment of research needs and opportunities. The areas selected were: Physical (i.e. Pore) Structure of Coal, Analytical Techniques, Instrumentation and Control, Modeling and Flow Theory, Materials Problems, Size-dependent Phenomena (i.e. size reduction of coal and coal/fluid interactions), and Catalysis. The bulk of this report then consists of these research assessments, which in these areas delineate the practically important and scientifically significant opportunities for physicists.

Our principal recommendations precede this Introduction, as part of the Executive Summary. They include both recommendations for specific priority research and for institutional arrangements necessary to get the most out of that research, specifically (1) establishment of a program for development and testing of theoretical models and process control techniques in coordination with requisite work on development of instrumentation, and (2) provision for a "bank" of well characterized coal samples. More detailed lists of recommendations are included as part of the research assessments for the individual technical areas.

\section{Organization of the report}

The remainder of the report presents the research assessments in the selected areas, including the background necessary to understand both motivation and the context of what is being recommended. The material is divided into two large sections (Sections III and IV), one dealing with research on coal itself, the other dealing with research pertinent to the technology of coal utilization and synthetic fuel production. On the assumption that the coal related background of many readers is limited, each section begins with a primer, i.e. the "coal primer" in Section III A and the "coal technology primer" in Section IV A. There is also an appendix on the amount of $\mathrm{CO}_{2}$ produced by various means of coal utilization or conversion.

The coal section deals with two research areas: physical (i.e. pore) structure of coal in III $B$ and analytical techniques in III $C$. The motivation for dealing with these topics is simple. To utilize coal most effectively, one wants to understand as much about coal as possible.

For example, in the view of advocates of direct liquefaction processes, the aim is to preserve as much as possible of the desirable molecular structure of the organic part of the coal, minimizing molecular rearrangements when it is converted into a liquid. That school of thought holds that this should be inherently more energy efficient than indirect processes, where one completely destroys the molecular structure of coal in creating a "syngas" mixture of $\mathrm{CO}$ and $\mathrm{H}_{2}$. Whether or not this expectation of the relative efficiency of direct liquefaction is ultimately vindicated, the development of efficient direct liquefaction processes requires knowledge of the molecular structure of coal; indeed, it requires knowledge of whether one should think of a molecular structure at all or should adopt some other heuristic picture for the chemical structure. Such need for knowledge of the chemical structure of coal will occur often in technology development. Clearly this requires analytical techniques. The problem is that coal is a highly variable conglomerate material composed of several distinguishable organic substances and minerals. Moreover, there is no "standard" coal, nor any obvious way to define one. The Analytical Techniques section addresses itself to the question of what the physicist can do, and what is needed to introduce new techniques in the face of these difficulties.

We have already mentioned above the key role played by the pore structure in 
controlling chemical reactions in coal. Diffusion through the pore system and the mechanism of adsorption at the pore surfaces indeed may limit the rates of such reactions. Methods for characterizing the pore structure, and the behavior of molecular species of interest within this structure, are discussed in the Pore Structure Section.

The "coal technology primer" of IV A is followed by the discussion of Instrumentation and Control in Section IV B. Control is necessary for safety and reliable operation under severe conditions involving extremely high temperatures and pressures and harsh physical and chemical environments, where the need for short response times often greatly compounds the difficulties. Also the instrumentation must be done in an economical way. Up until now, instrumentation design efforts have been concentrated on needs of control for safety and process operations. Another main objective of instrumentation design should be for the data acquisition necessary for realistic modeling of conversion and combustion processes as described in Section IV C. Development of such predictive theoretical models is important to the economic improvement of coal technology, particularly in view of its sensitivity to process details. One must capture these details, for example the effects of mineral content, in the complicated set of differential equations used to formulate the model for some process, and then find a tractable way to solve these equations, again preserving the details of interest.

Single phase turbulent flow and multi-phase flow are pervasive features of processes of interest. For example, high temperature, high velocity slurries of coal particles in fluids, as commonly occur in coal conversion systems, involve problems of two-phase flow. Development of the theory of flow phenomena, also discussed in IV $C$, is central to improvement of modeling and consequent improvement of the processes.

Finding durable materials of construction, able to provide desired properties, and assure adequate lifetime, under the harsh operating conditions of coal utilization and synthetic fuel technology, may be the key to economic viability. To be economically successful, a plant must not merely work, it must continue to work, reliably and at reasonable maintenance expense. The pertinent materials requirements suggest research needs and programs that are discussed in Section IV D.

Coal utilization typically involves particle size reduction. Also interactions with fluids (whether native to the coal or added for transportation, processing, or during size reduction) depend on phenomena at the coal/fluid interface and hence are also likely to depend significantly on coal particle sizes. Several topics concerned with coal preparation and coal/fluid mixtures are treated under the category of "size-dependent phenomena" in Section IV E.

Effective catalysts are important for an economically viable synthetic fuel production technology, because of the desire to perform the necessary reactions rapidly under "mild" conditions of temperature and pressure. Of course, in current practice, these conditions are not at all mild, so progress in catalysis could have very substantial economic impact. Also, catalysis plays a key role in product selectivity, e.g. getting more of the medium weight hydrocarbons that constitute gasoline and less of heavier or lighter hydrocarbons. Maintaining the effectiveness of catalysts is important because catalysts can be very expensive; and therefore understanding and combating "poisoning" of catalysts is important. Questions of catalysis relate to research interests of physicists in several ways, particularly in the area of electronic structure at surfaces and interfaces, and in small metallic particles. Such questions are discussed in Section IV F.

There is, of course, much interplay between research questions in the broad research areas discussed in Sections III and IV. We already have emphasized the link between instrumentation development for data acquisition and modeling. Technical problems in development of analytical techniques for learning about the chemical structure of coal are sometimes related to those of instrumentation for process control and understanding. A number of questions of catalysis depend on the pore structure of coal, or involve materials, such as zeolites, themselves having a (much more well defined) pore structure; and comminution (size reduction) of coal and coal/fluid interactions also depend upon the physical structure of coal. Other examples of interrelationships between the different areas will be apparent to the reader.

In this report the focus is on research topics pertinent to better characterization of coal and to improvement of coal utilization 
processes. Although the Panel was aware of the importance of environmental effects in coal utilization, we have not dealt with questions of environmental impact. Therefore research topics of environmental effects concomitant to the use of coal such as atmospheric effects, global heating, acid rain, etc. have not been addressed. (That is, we have not dealt with impacts of these effects. The technical topics we have treated rather deal with monitoring and controlling such emissions.) Such topics form research areas of their own and require considerations differing in generic as well as detailed character from those considered here. (See discussion in Office of Technology Assessment, 1979; Green, 1980; Wilson, 1980; Landsberg, 1979; National Academy of Sciences, 1980) However, it is necessary to recognize the coupling between these research areas and the subjects of this report since different coal utilization processes produce, in addition to desirable forms of energy and fuel, various yields of nitrogen oxides, sulfur oxides, aerosols, respirable particles, small residues of natural radioactivity, and carbon dioxide.

In particular, $\mathrm{CO}_{2}$ is an inevitable emission in the utilization of fossil fuels. The amount of $\mathrm{CO}_{2}$ is inversely related to the thermal efficiency. This means that if one is concerned about $\mathrm{CO}_{2}$ production, one has no alternative to $a$ deep concern for thermodynamic efficiency in every aspect of hydrocarbon utilization. In Appendix I, we present a comparison of various coal utilization schemes as sources for $\mathrm{CO}_{2}$. Our analysis leads to some numbers that ${ }^{2}$ give a feeling for the amounts of $\mathrm{CO}_{2}$ that would be produced by synthetic fuel technology as presently contemplated.

\section{General remarks}

We have recognized many specific difficulties needing solution, or uncertainties needing clarification, for the furtherance of coal utilization and synthetic fuel production technology. However, we realize that some of these, for example those having to do with erosion and corrosion effects on materials of construction, are scarcely new. In fact the Department of Energy, both through ASFE and OER, has long maintained research programs in some of these areas - - as have several other federal agencies and industry. However it should be emphasized that coal and its associated technologies present many of these problems and difficulties in extraordinarily severe form, and that extraordinary efforts are needed. It is important for physicists to bring to bear new perspectives for solving these problems.

Much invention is needed (e.g. something better than lock-hoppers for dry materials feeding). And though invention often results indirectly from research it is not an automatic consequence -- and the direction of invention can scarcely be anticipated on the basis of the direction of research. Several other Studies and Reports have remarked on the fact that many problems probably will come into clear focus, only as a result of operating experience with rather large scale facilities, i.e. large pilot (about 250 tons/day) or demonstration-size (thousands of tons/day) facilities.

In addition, much basic scientific research is needed, including that by physicists. The perception of this need was the initiating impetus to this report, and our efforts have reaffirmed this thesis. Very many of the open questions in coal utilization and synthetic fuel development are at the limits of present physical understanding, e.g., of surfaces and of multi-phase flow. Moreover, the techniques physicists are accustomed to employ -- lasers, NMR, neutron scattering, etc. -- are hopeful tools for answering those problems. We especially stress that coal researches by physicists can extend basic knowledge in directions which other physicists will find instructive and will be happy to find published in contemporary physics journals.

To create an atmosphere conducive to invention and solutions to problems it is important to expose the whole area of coal technology to a broader technical community. We believe that the government agencies and professional societies should do as much as possible to provide avenues of publication and recognition and to strengthen communication among scientists and engineers in all disciplines working on coal. The holding of meetings and topical conference, as already done under the sponsorship of DOE and APS among others, is one example of desirable action.

\section{References}

Energy Research Advisory Board, 1980 , Research and Development Needs in the 
Department of Energy, Interim Report of the Research and Development Panel, Energy Research Advisory Board, September.

Fossil Energy Research Working Group, 1979, Assessment of Long-Term Research Needs for Coal-Gasification Technologies, Fossil Energy Research Working Group, S. S. Penner - Chairman, MITRE Technical Report MTR-79W00160.

Fossil Energy Research Working Group, 1980, Assessment of Long-Term Research Needs for Coal-Lique faction Technologies, Fossil Energy Research Working Group, $S$. S. Penner - Chairman.

Gas Research Institute, 1980, Basic Coal Sciences Project Advisory Committee Report, Gas Research Institute, Chicago, Illinois.

Gorbaty, M. L., F. J. Wright, R. K. Lyon, R. B. Long, R. H. Schlosberg, Z. Baset, R. Liotta, B. S. Silbernagel, D. N. Neskora, 1979, Science 206, 1029.

Green, A. E. S., 1980, Coal Burning Issues, edited by A. E. S. Green (University Press of Florida, Gainesville).

Grimes, W. R., G. D. Brunton, H. D. Cochran, Jr., J. R. Hightower, Jr., J. S. Johnson, Jr., I. L. Thomas, J. A. Watson, and F. W. Wiffen, 1980, "Assessment of Basic Research Needs and Priorities in Support of Fossil Energy" in Basic Research Needs in Seven Energy Related Technologies, DOE-ER-0060.

Keller, O. L., 1979, ORNL Coal Chemistry 1979, ORNL 5629.

Landsberg, H. H., 1979, Energy: The Next Twenty Years; Report by a study group sponsored by the Ford Foundation and adminstered by Resources for the Future; H. H. Landsberg, Chairman (Ballinger Publishing Co., Cambridge).

National Academy of Sciences, 1979. Some Aspects of Basic Research in the Chemical Sciences, National Academy of Sciences, 1979.

National Academy of Sciences, 1980, Energy in Transition 1985-2010, Final report of the Committee on Nuclear and Alternative Energy Systems, National Academy of Sciences (W. H. Freeman and Company, San Francisco).

Office of Technology Assessment, 1979, The Direct Use of Coal, Congress of the United States, Office of Technology Assessment (Superintendent of Documents, U.S. Government Printing Office, Washington, D.C., Stock No. 052-003-00664-2).

Wilson, C. L., 1980, Coal -- Bridge to the Future, Carroll L. Wilson, Project Director, Report of the World Coal Study (Ballinger Publishing Co., Cambridge). 
III. RESEARCH IN COAL

\section{A. PRIMER ON COAL}

\section{Origin and petrography}

The term "coal" is applied to a very wide range of substances. All are carboniferous solids formed over geological periods by the metamorphosis of vegetable matter under heat and pressure. Coal in the words of one authority, (Francis, 1954), is:

" . . a compact stratified mass of mummified plants which have been modified chemically in varying degree, interspersed with smaller amounts of inorganic matter."

This having been said, one should be prepared from the outset for the fact that coal displays on every scale - - atomic, microscopic*, and macroscopic -- an intrinsic heterogeneity which reflects both the natural biological variability of vegetation and the diversity of geologic histories resulting in coalification.

The starting matter is chemically and physically heterogeneous -- containing wood, bark, leaves, needles, pitch, spores, algae, etc., and all their diverse chemical constituents such as resins, waxes, lignins, hemi-cellulose, cellulose, etc. Over periods ranging from 60 to 200 million years, moreover, the various coals have experienced an extremely wide range of differing geologic histories. Processes of coalification are complex and poorly understood. The transition from biological processes (e.g. decomposition mediated by microbes) to purely physical processes cannot be unambiguously reconstructed. At present, it is not possible to discuss the structure of coals in terms of known transformations of known precursors. (Flaig, 1966, Stach, 1975)

The conglomerate character of coal is visually apparent under the petrographer's microscope. (Petrakis, 1980) The carbonaceous (organic) material is composed of several distinguishable substances called "macerals" which are disposed in irregularly shaped domains. Macerals may be classified into about four principal easily distinguishable varieties, with numerous finer distinctions possible. The domains range in size from a

* It is useful, in the case of coal, to recognize a "microscopic" scale intermediate between the atomic and the macroscopic, since many of the interesting features fall in the range of a few microns. few microns to the macroscopic. Different macerals are most immediately distinguishable because their optical reflectivities differ, and they are found also to differ chemically and in regard to physical characteristics other than reflectivity. Different macerals are believed to arise from different constituents of the original vegetable matter. Coal also contains inclusions of inorganic matter; these are for the most part standard minerals, not unique to coal, and well studied in other contexts. They do have special significance for the chemical and physical processing of coal, however.

The serious student of coal must be prepared to come to grips with non-idealized reality. It is not possible to commence the scientific study of coal by "purifying" it. In fact, neither the notion "pure" nor the concept "perfect", as familiar to solid state physicists and chemists, is relevant in the case of coal. The nearest possible thing to a classical (pure substances) approach is to study the properties of segregated macerals, one variety at a time. The need to do this imparts some importance to the development of efficient techniques for separating and sorting macerals (Dyrkacs and Horwitz, 1981; Kroger, 1957; Goloushin, 1959).

\section{Classification and nomenclature}

Difficulties and ambiguities of classification are unavoidable for substances as varied and complex as the family of coals. Indeed, no system has yet been devised which satisfactorily reflects the variations of atomic arrangement of different coals or their behavior as chemical reactants. (The prerequisite fundamental chemical and physical knowledge is still lacking.)

The traditional schemes of classification are according to "rank" and "type." Each (in several versions) is in current use. (Neavel, 1978; Berkowitz, 1980) Together, they furnish a scheme of two independent dimensions -- in the sense that a given rank may find representation in coals of several types and vice versa.

Rank

Assignment of rank provides a gross average characterization of a coal indicating primarily its heating value as a fuel in direct combustion; it is not concerned with micro-structure. Lignite, sub-bituminous 


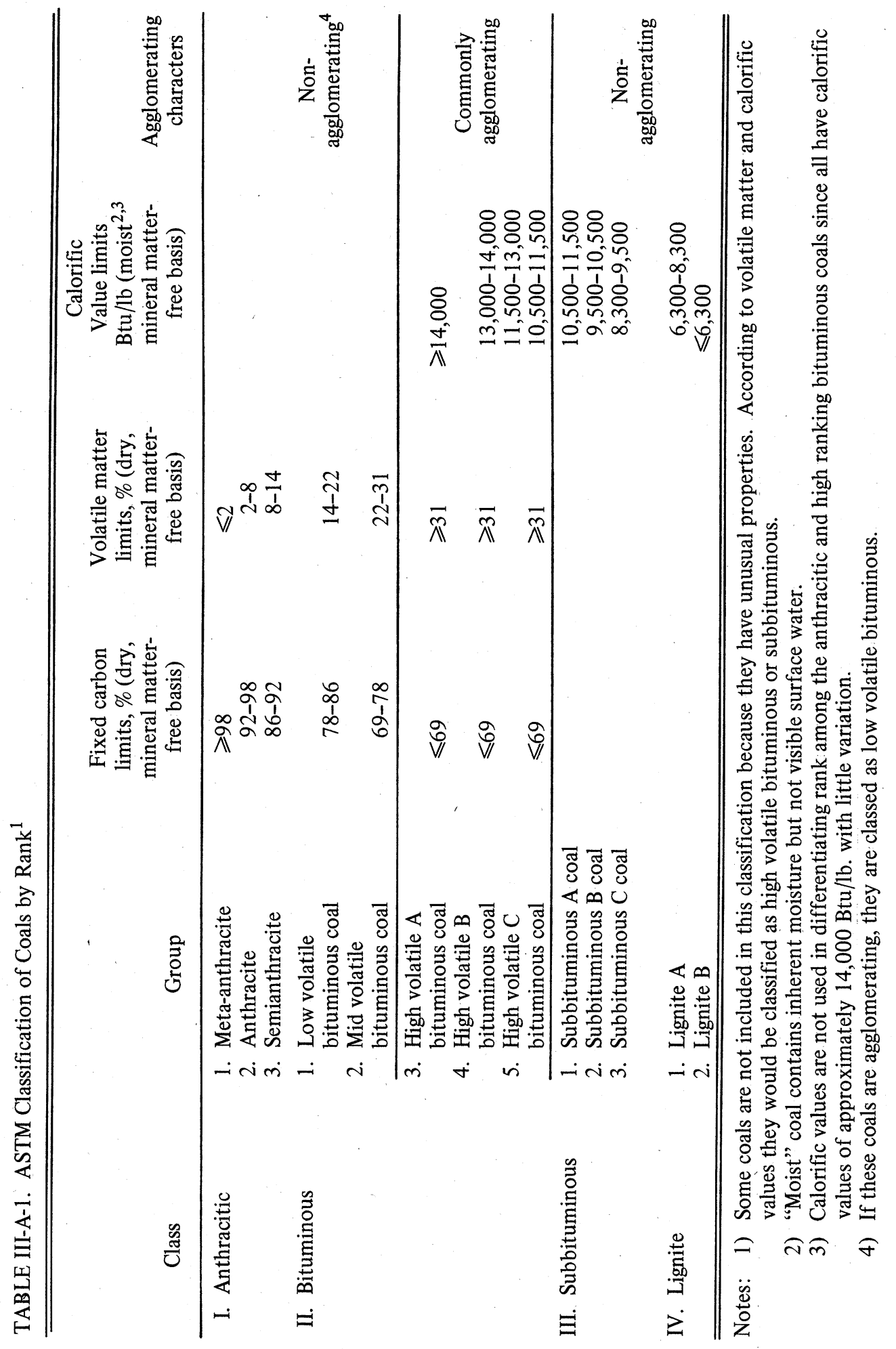

Rev. Mod. Phys., Vol. 53, No. 4, Part II, October 1981 
TABLE III-A-2. Comparison of Coal Macerals, Thiessen (U.S.) and Stopes-Heerlen (Europe)

\begin{tabular}{|c|c|c|}
\hline Thiessen system & & Stopes-Heerlen system \\
\hline anthraxylon and humic matter & vitrinite & $\begin{array}{l}\text { - colinite } \\
\text { - telinite }\end{array}$ \\
\hline $\begin{array}{l}\text { spores and pollen } \\
\text { cuticles } \\
\text { algal remains } \\
\text { resinous and waxy substances }\end{array}$ & $\begin{array}{l}\text { exinite } \\
\text { or } \\
\text { liptinite }\end{array}$ & $\begin{array}{l}\text { - sporinite } \\
\text { cutinite } \\
\text { alginite } \\
\text { - resinite }\end{array}$ \\
\hline $\begin{array}{l}\text { opaque matter, brown matter } \\
\text { fusain } \\
\text { fusain and brown matter } \\
\text { fusain and brown matter }\end{array}$ & inertinite & $\begin{array}{l}\text { micrinite } \\
\text { fusinite } \\
\text { semi fusinite } \\
\text { sclerotinite }\end{array}$ \\
\hline
\end{tabular}

coal, bituminous coal, and anthracite are the main rank classes. Peat is considered a coal precursor. Several systems exist for assigning rank to coal. The one currently used in the United States (ASTM-D-388-66) is shown in Table III-A-1. The parameters used to differentiate between ranks are: 1) "fixed carbon", (FC) when the value of this is greater than 69 per cent (fixed carbon is the dry, mineral free residue from a "volatile matter" determination). Volatile matter (VM) is defined as the per cent by weight, lost by heating a dry sample to $950 \mathrm{C}$ for seven minutes in the absence of air (thus on a dry, mineral-matter-free basis: $F(+V M=100) ; 2$ ) when the fixed carbon value is less than 69 per cent, differentiation is by calorific (heating) value and/or agglomeration (the characteristic of forming a coke button in the volatile matter test).

Geologically, the progression of ranks from lignite to anthracite may be viewed as an ordering according to increasing degree of metamorphosis or coalification of the original vegetable matter. (Parks, 1963; Berkowitz, 1980) Historically, however, rank was originally devised to differentiate between coals to be used in coking and combustion. Even for these purposes rank alone does not suffice and must be augmented with "grade" information (Neavel, l978) specifying the mineral matter content, sulfur content, and ash fusion temperature -- qualities which do not vary systematically with rank. Different coals, moreover, exhibit different swelling, softening, agglomeration, and "caking" behaviors which are important in coke making and other conversion technologies (Beimann et al., 1963; Thompson, 1981).

\section{Type}

Type classification accords recognition to coal's small scale heterogeneity and to its petrographic characterization - - type being now assigned according to the proportions in which the various maceral varieties are present. (An obvious prerequisite is to have catalogued and described the most common varieties of maceral.) Typing according to maceral content dates back to Stopes (1919) and Thiessen $(1920,1938) . *$ Stopes based his classification on observations using reflected light, while Thiessen based his on observations of thin sections by transmitted light. Table III-A-2 gives a comparison of terms used in the two systems of maceral definition and identification.

\section{Chemical structure}

Coal chemistry is faced with formidable tasks if coals are ever to be described at the molecular level, but the potential payoffs may well be commensurate with the difficulty of the task. Presently available synthetic fuel processes represent a start on science based refinement and extension of technologies first conceived decades ago. There is little to indicate that the processes are efficient compared to others that might be developed. A better knowledge of the molecular structure of coal might suggest completely new reactions, catalysts, or processing conditions.

* The nomenclature of coal types is much older and goes back to earlier type classifications based upon visible differences and upon properties like texture and mode of fracture. 
Despite the difficulty of the problem some progress has been realized in recent years as new analytical techniques from physics and chemistry have been applied to coal (Karr, 1978, 1979). There remain, however, promising scientific tools which have yet to be applied to coal including some, at least, which could be extended or modified in ways appropriate to the particular problems of coal. Section III $C$ of the report is devoted to a more extensive assessment of these questions.

Studies of segregated maceral varieties reveal that not only their optical properties but also their elemental compositions, densities, and microscopic physical structures differ. See Table III-A-3. One sees here an important general point, more fully discussed in Section IV-A: even the highest hydrogen content noted (that for exinite) corresponds to an H/C ratio of roughly 0.9 , whereas high grade liquid transport fuels typically exhibit ratios in the range 1.9 to 2.0 . In reaction studies different macerals respond differently to hydrogenation, solubilization, pyrolysis, etc. (Thiessen and Francis, 1929; Given, 1975) and even a single maceral variety does not show the constant, well defined properties that would be characteristic of a pure substance.

Structure at the atomic level is difficult to investigate because coal is insoluble in common solvents, non-crystalline, and nearly opaque. It is easily pyrolzed (decomposed by heat in the absence of oxygen), but coal as such does not exist in the vapor state. Although coal in some ways resembles a polymer (Lucht and Peppas, 1981) and exhibits some polymer type behavior, it is not a true polymer since it has no known repetitive structural unit. Even a single maceral is a mixture of macro-molecules. Thus coal presents several obstacles to the deployment of modern chemical and physical instrumentation in the customary ways. And despite a great deal of research and some good results already accomplished, knowledge of coal structure at the atomic level remains unsatisfactory. Section III-C of the report gives an assessment of current prospects in this area.

It does seem clear that there is no unique "structure of coal"; each seam and, probably, each sample is different in its composition, structure, and properties. One expects that the macromolecular entities in coal are composed of sub-units some of which may themselves be released more or less intact upon mild dissolution or other chemical treatment which only affects the weakest bonds or specific types of bonds in the structure. In this view a credible aspiration for the description of coal at the atomic level would be to develop a statistical characterization of :

- molecular size distribution

- sizes and types of sub-units (including such features as their aromaticity and chemical functionalities)

- degree and types of crosslinking between sub-units

To be useful such a characterization must specify distributions and not merely averages, and the cross-linking information must include some treatment of steric effects and three dimensional structure.

Such descriptions have been offered by Fuchs and Sandhoff (1942), Given (1961), and

TABLE III-A-3. Chemical Composition and Physical Properties of Macerals of a High Volatile A Bituminous Coal.

\begin{tabular}{|c|c|c|c|c|c|c|c|c|c|}
\hline \multirow[b]{2}{*}{ Component } & \multicolumn{5}{|c|}{ Composition, wt. $\%$} & \multirow[b]{2}{*}{$\begin{array}{c}\text { Density } \\
\mathrm{g} / \mathrm{cm}^{3}\end{array}$} & \multirow[b]{2}{*}{$\begin{array}{c}\% \text { Reflectance } \\
\text { in oil }\end{array}$} & \multirow[b]{2}{*}{$\begin{array}{l}\text { Refractive } \\
\text { index }\end{array}$} & \multirow[b]{2}{*}{$\begin{array}{c}\text { Surface } \\
\text { area } \\
\left(\mathrm{m}^{2} / \text { gram }\right)\end{array}$} \\
\hline & $S$ & $\mathrm{~N}$ & $\mathrm{O}$ & $\mathrm{C}$ & $\mathrm{H}$ & & & & \\
\hline Exinite & 0.7 & 1.1 & 5.5 & 86.2 & 6.5 & 1.21 & 0.32 & 1.68 & 2.5 \\
\hline Vitrinite & 1.0 & 1.6 & 8.0 & 84.1 & 5.3 & 1.29 & 0.91 & 1.81 & 2.1 \\
\hline Micrinite & 0.6 & 0.7 & 8.0 & 85.9 & 4.8 & 1.32 & 1.61 & 2.01 & 4.6 \\
\hline Fusinite & 0.4 & 0.6 & 4.3 & 91.5 & 3.2 & 1.48 & 2.65 & 1.91 & 9.8 \\
\hline
\end{tabular}


Wiser (1978). Figure III-A-1 depicts Wiser's representation; it is intended not to depict an actual coal stucture, but rather as a heuristic aid to show the main types of structural units, functional groups, and linkages that might be present in a typical bituminous coal. Undoubtedly an actual structure is three dimensional and variable. A three dimensional heuristic model very closely based on Wiser's representation is shown in Figure III-A-2. To obtain this "sterically permissible" space-filling structure it was necessary to modify Wiser's representation only slightly (Spiro, 1981). of course representations of this type, if they were to be extended to coals of other ranks, would have to be constructed of correspondingly different sub-units as indicated in Figure III-A-3 (Wender, 1976).

Very concise discussions of coal chemistry and structure in relation to coal technology may be found in Gorbaty et al, 1979 and Gas Research Institute, 1980. A wider range of topics in coal science is discussed in Lowry, 1963; Elliott, 1981; van Krevelen and Schuyer, 1957; van Krevelen, 1957; Cooper, 1978; and Cooper and Petrakis, 1981.

\section{Physical structure}

In terms of its physical structure, coal has been found to be a heterogenous solid containing capillaries, cracks, and open and closed pores (with and without occluded matter) in at least three broad ranges of characteristic dimension. The pores have been categorized as: macropore ( $200 \AA)$, mesopore $(20-200 \AA)$, and micropore ( $\leq 20 \AA)$ (Grimes, 1980). Some coal samples exhibit specific internal surface area as high as several hundred meter /gram. (Results differ markedly from one coal to another and also depend significantly on the measurement technique chosen.) The origin or cause of this porosity, although it is of technological as well as scientific interest, is not yet known (Gay, Nandi, and Walker, 1972).

The crack and pore structure is of great practical importance since it influences the access and egress of reagents and reaction products in various chemical processes and the detailed mechanism of combustion of small particles (Larsen, 1981). (See Section IV B) In fact it appears that for many industrial processes these factors and the presence of

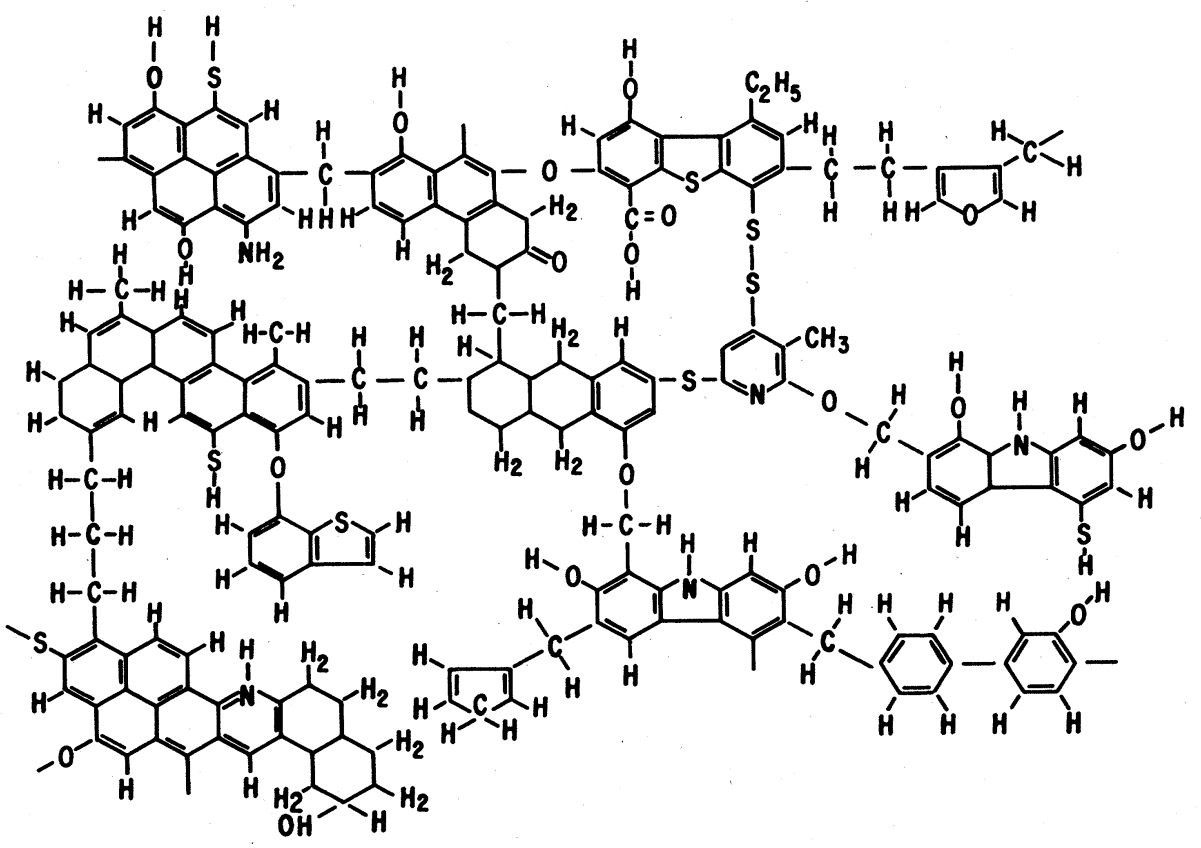

FIG. IIIA-1. Schematic representation of structural groups and connecting bridges in bituminous coal (Wiser, 1978). 


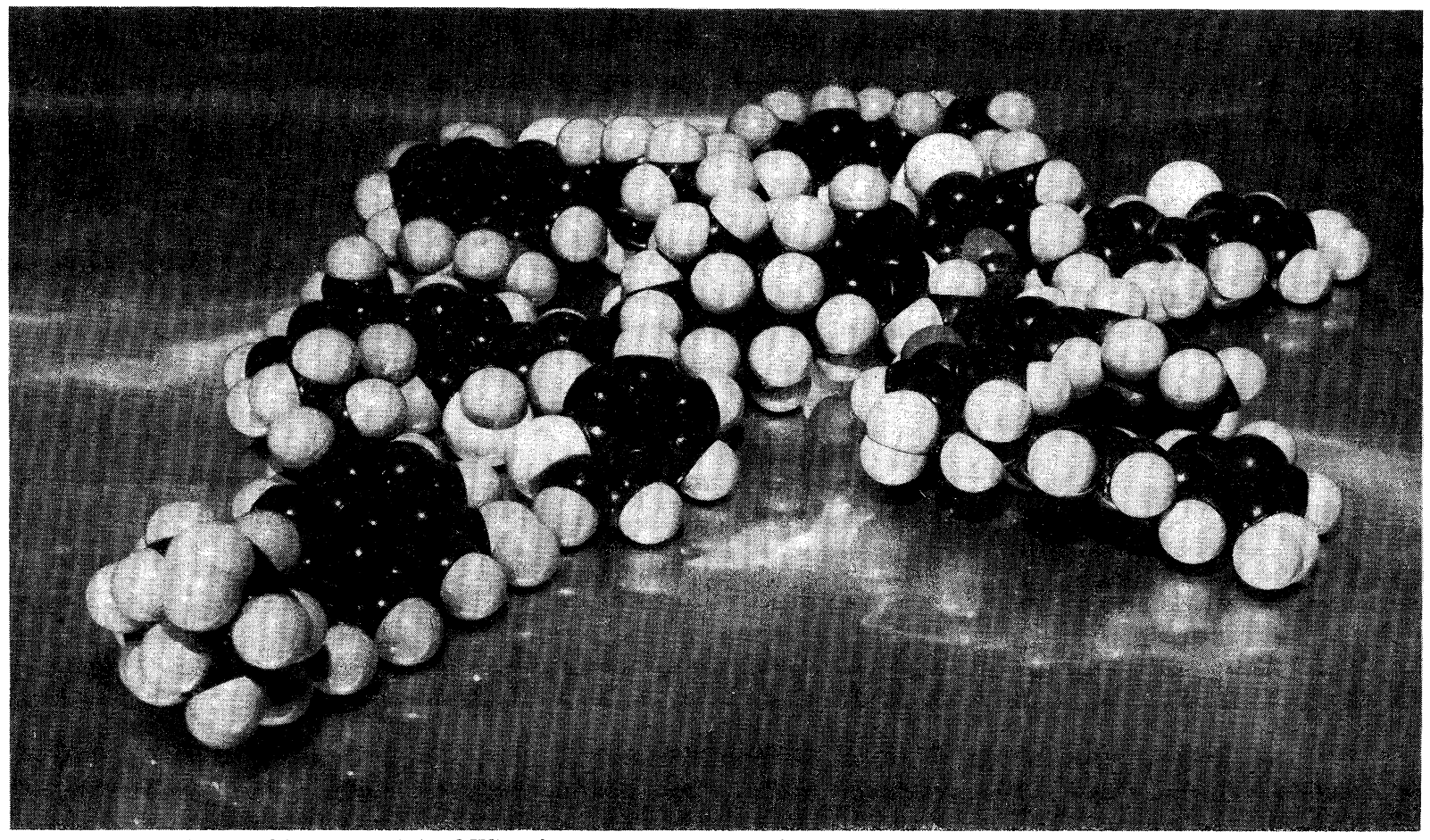

FIG. IIIA-2. Space filling model of Wiser's representation of bituminous coal (Spiro, 1981).

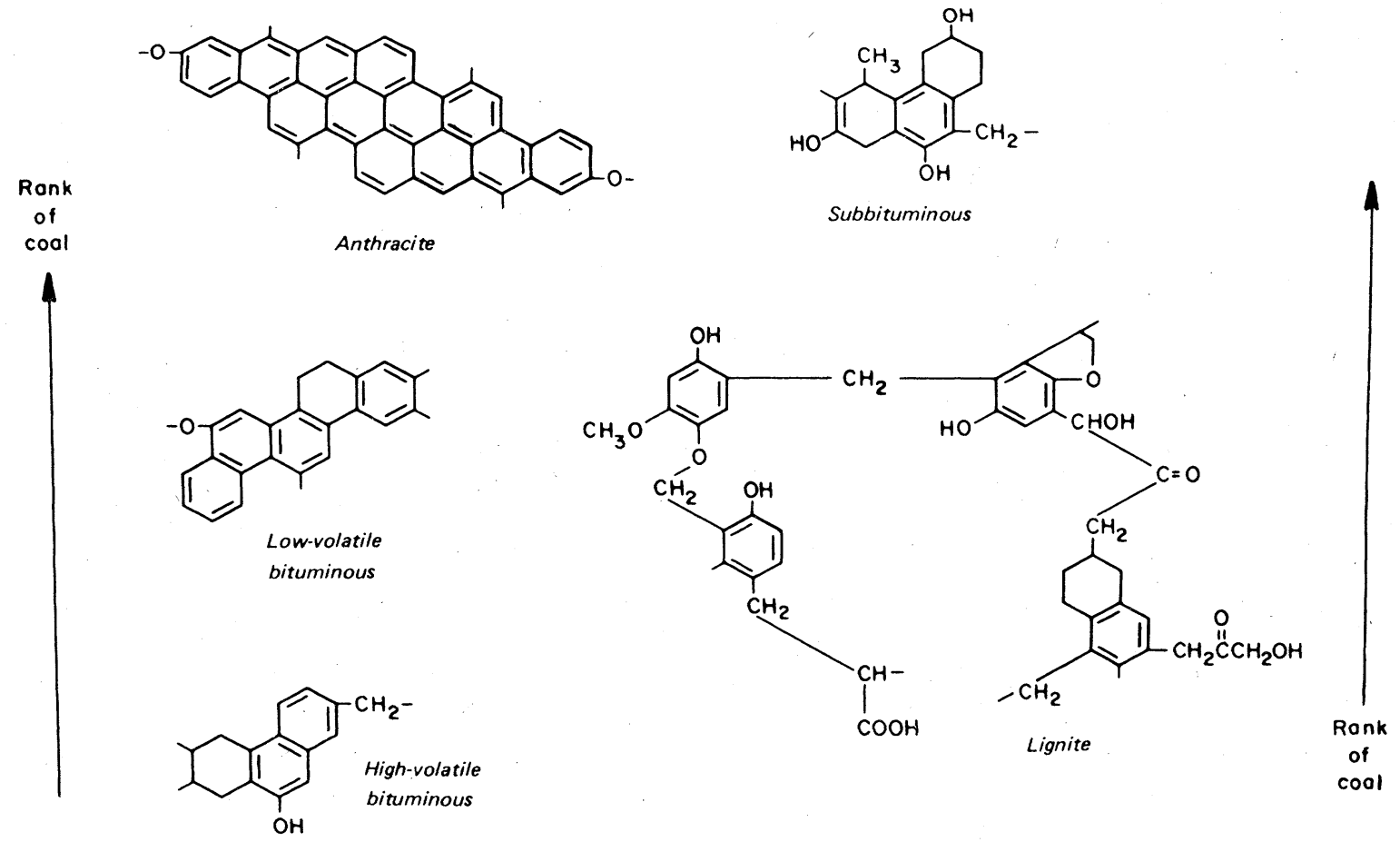

FIG. IIIA-3. Representative partial structures of different ranks of coal (Wender, 1976). 
occluded moisture, gases, and minerals may be more important determinants than the intrinsic chemistry. Porosity is especially amenable to investigation by physics techniques and is discussed in Section III B.

\section{Stability}

As taken from the mine, many coals, especially those of lower rank, are unstable in the presence of air. Their qualities as fuel or as input to chemical processes are changed by slow oxidation and irreversible water migration; some even undergo spontaneous combustion initiated by heat of adsorption and other surface reactions. These phenomena are of some consequence for the overall design of coal utilization systems, and they must be very carefully controlled in any attempt to provide standard samples for laboratory research. The matter of standard samples is discussed in Section III $C$, and is the subject of one of our Principal Recommendations as discussed in Section I.

\section{References}

Beimann, W., H. S. Auvil, and D. C. Coleman, 1963, Chapter 11 in Chemistry of Coal Utilization, (Supplementary Volume) edited by H. H. Lowry, John Wiley and Sons, New York.

Berkowitz, N., 1980, Introduction to Coal Technology, Academic Press.

Cooper, B. R., 1978, Editor, Scientific Problems of Coal Utilization, Technical Information Center, U.S. Dept. of Energy. (Available as CONF-770509 from National Technical Information Service, Springfield, VA 22161).

Cooper, B. R. and L. Petrakis, 1981 , Editors, Chemistry and Physics of Coal Utilization - 1980, American Institute of Physics Conference Proceedings No. 70.

Dyrkacz, Gary and E. P. Horwitz, Fuel, in press.

Elliott, M. A., 1981, Editor, Chemistry of Coal Utilization, Second Supplement, Wiley, New York.

Flaig, W., 1966, in Coal Science, P. Given, ed., (Am. Chem. Soc., Wash. D.C.) p. 58.

Francis, Wilfrid, 1954, "Coal, Its Formation and Composition", Edward Arnold (Publishers) Ltd. p. 1.

Fuchs, W. and A. G. Sandhoff, 1942, Ind. Eng. Chem., 34, 567.
Gan, H., S. P. Nandi, and P. L. Walker Jr., 1972, Fuel 51, 272.

Gas Research Institute, 1980, Basic Coal Science Project Advisory Committee Report, Gas Research Institute, Chicago, IL.

Given, P. H., 1961, Fuel 39, 147, 1960 and $40,427$.

Given, P. H., D. C. Cronauer, W. Spackman, H. L. Lovell, A. Davis and B. Biswas, 1975, Fuel 54, No. 1, 40-49.

Goloushin, N. S., 1959, J. Appl. Chem. USSR 32, 1969.

Gorbaty, M. L., F. J. Wright, R. K. Lyon, R. B. Long, R. H. Schlosberg, Z. Baset, R. Liotta, B. S. Silbernagel, and D. N. Neskora, Science 206, 1029.

Grimes, W. R., 1980, "The Physical Structure of Coal" in Advances in Coal Chemistry, Vol. 1, Chapter 2, Academic Press.

Karr, Clarence Jr., 1978, 1979, Analytical Methods for Coal and Coal Products, 3 v., Academic Press.

Kroger, Carl,'1957, Gluckauf, Vol 93, p. 122.

Larsen, J. W., 1981, pp. 1-27 in Chemistry and Physics of Coal Utilization-1980, edited by B. R. Cooper and L. Petrakis, American Institute of Physics Proceedings No. 70, New York.

Lowry, H. H., 1963, Editor, Chemistry of Coal Utilization, Supplementary Volume, Wiley, New York.

Lucht, L. M., and N. A. Peppas, 1981, pp. 28-48 in Chemistry and Physics of Coal Utilization -1980 , edited by B. R. Cooper and L. Petrakis, American Institute of Physics Proceedings No. 70, New York.

Neavel, R. C., 1978, pp. 77-79 in Scientific Problems of Coal Utilization, edited by B. R. Cooper, U. S. Dept. of Energy Symposium Series.

Parks, B. C., 1968, Chapter 1 in Chemistry of Coal Utilization, edited by H. H. Lowry, John Wiley and Sons, New York.

Petrakis, L. and D. W. Grandy, 1980, Journal of Chemical Educ., 57 \#10, 683.

Spiro, C. L., 1981, to appear in Fuel.

Stach, E., M. - Th. Mackowsky, M. Teichsmuller, G. H. Taylor, D. Chandra, and R. Teichsmuller, 1975, Textbook of Coal Petrography, English trans. by D. G. Murchison et al., (Gebruder Borntraeger, Berlin, Stuttgart).

Stopes, M. D., 1919, Proc. Roy. Soc. (London) B90, 470-487.

Thiessen, R., 1920, "Coal Age" 18 , 
$1183-1189,1223-1228$ and 1275-1279.

Thiessen, R. and W. Francis, 1929, U.S. Bur. Mines Tech. Paper 446, 27pp.

Thiessen, R., G. C. Sprunk and H. J. O'Donnell, 1938, U.S. Bur. Mines I.C. 7021, 1939; Fuel 17, 307-315.

Thompson, R. R., 1981 , pp. 49-65 in Chemistry and Physics of Coal Utilization -1980, edited by B. R. Cooper and L. Petrakis, American Institute of Physics Proceedings No. 70, New York.

van Krevelen, D. W. and J. Schuyer, 1957,
Coal Science, Elsevier, Amsterdam.
$\mathrm{v}$ a $\mathrm{Krevel}$ e $n$ D. W. , 1957 , Coal-Typography-Chemistry-

Physics-Constitution.

Wender, I., 1976, Catalytic Synthesis of Chemicals from Coal, Catalysis Reviews Science and Engineering, 14(1): 101, Fig. 2.

Wiser, W. H., 1978, pp. 219-236 in Scientific Problems of Coal Utilization, edited by B. R. Cooper, U.S. Dept. of Energy Symposium Series. 


\section{B. PORE STRUCTURE OF COAL}

\section{Introduction}

A number of important physical and chemical properties of coal are intimately connected with, and sometimes uniquely influenced by, the pore structure of coal (Walker, 1980; Grimes, 1980). Among these are effects relating to adsorption, absorption, diffusion, mass transport, catalytic and combustion processes. Effective coal utilization requires a much improved understanding both in the physical description of the pore structure, and in the way that pore structure influences how chemical reactions occur for coal.

As stated by Grimes, 1980, "...details of the pore structure - influence the behavior of coal more directly than does virtually any other property.". This is readily appreciated with the realization that it is only through the surfaces, especially the internal pore surfaces (the integrated value of which can range from 10 's to over $200 \mathrm{~m}^{2} / \mathrm{g}$ ) that the coal comes into contact with the external environment.

Certain coals behave like deformable viscoelastic colloids. Because of this, the physical characteristics of coal, and therefore its (slit-shaped) pores, will change and alter significantly as a function of temperature, pressure, and the intrusion of molecular species. For example, the well known swelling of bituminous coals in the presence of water is an illustration of this.

Below we give some examples in which the pores in coal play an important role in its utilization:

Coal Gasification: The reactivity in coal gasification processes depends on the extent of the microporosity in coal. However, at the required elevated temperature and during the heating process, the microporosity of the coal undergoes drastic changes, with the nature of the changes significantly depending on the type of coal used. For sub-bituminous coal and lignites the elevated temperature results in the removal of volatiles in coal and consequently both an increase in average pore size of the open microporosity and the opening up of previously sealed pores. For most bituminous coals, on the other hand, much of the open porosity is lost when the coal goes through a plastic phase upon heating to gasification temperatures. Treatment of coal (e.g., addition of oxygen) during the heating process can significantly alter the porosity, and therefore can increase reactivity in the coal gasification process.

Coal Liquefaction: In some direct liquefaction processes, hydrogen is added to molecular fragments formed by thermal bond breaking, which occurs at temperatures of about $400 \mathrm{C}$, through the use of a donor solvent. The microporosity in coal enables the donor solvent to come into close contact with, and donate hydrogen to, the free bonds of the molecular fragments. This inbibition through the micropores (of diameter 20 or $30 \AA$ and less) must occur sufficiently rapidly with increasing temperature so that the process is complete before the thermal plasiticity of the coal leads to the closing of the micropores.

Coai/Gas Interface: One of the prominent coal/gas interfacial effects is the production and retention of methane gas in coal seams. The amount retained and its dependence on temperature and pressure will be relevant both in the safety aspect of coal mining operations and in the prospects for methane fuel gas extraction in underground mines.

Coal/Liquid Interface: In the important area of long distance coal transport, a recent development is the coal slurry pipeline. In the case of coal/water slurries, the coal particles are suspended in water to make them "pumpable", but water inside coal pores will decrease the efficiency of coal combustion. Slurries involving other fluids, such as methanol, liquid carbon dioxide, or oil, may be of interest under certain circumstances. Another area where the coal/liquid interface comes into play is in wetting and floation, of importance in coal preparation and cleaning (Taylor et al., 1981). (See IVE for a further discussion of coal/liquid interface phenomena.)

\section{Research areas}

\section{a. Quantitative characterization of coal pore size distribution}

There has been considerable research in the charactertization of the internal (i.e. pore) structure in coals. The prevalent 
methods used are "Molecular Methods" -- the use of molecules as probes. This includes the techniques of unsteady-state diffusion of gases, gas adsorption, and gas and liquid displacement (Walker, 1980; Grimes, 1980). These techniques do not yield unique values of surface area and pore volumes, but depend on the molecular probes used, e.g., pore volumes using helium and mercury as displacement fluids are quite different, presumably because mercury even at a pressure of $60 \mathrm{kbars}$ does not penetrate pores of less than $200 \mathrm{~A}$ diameter. (In fact, even different gases, say carbon dioxide and nitrogen, give different pore volumes.) The origin of this nonunique measurement of internal surface area arises from a combination of several factors: the size of molecular probe, the influence of pore surfaces on the behavior and volume of the probing molecule, and the influence of the probing molecules on the pore volume it is supposed to measure. In addition, molecular probes can only measure the volume of pores that are part of a network that communicates to the external surface. Molecular probe techniques generally yield integrated internal pore surface area. By measuring with different probes, with differing abilities to penetrate pores of varying size, one can develop models of the pore size distribution (Walker, 1980; Grimes, 1980).

The influence on the pore structure of molecular probes can be avoided by the use of nonintrusive physical probes, which have other advantages as well. Small angle $x-r a y$ scattering (SAXS) (Chiche et al., 1965; Kalliat et al., 1981; Lin et al., 1978; Schelton and Hendricks, 1978; Schmidt et al., 1981) is such a technique, and shows promise for determing the size distribution of the pores. Clearly, the SAXS methods is capable of probing all pores, including sealed as well as open (i.e. part of network extending to outer surface) pores. A great advantage of SAXS is the ability to perform such measurements under different temperature and pressure conditions, thus making it possible to follow changes in the pore structure dependent on these conditions. The availability of powerful synchrotron radiation X-ray sources, such as the forthcoming National Synchrotron Light-Source Facility at Brookhaven National Laboratory, enhances the possibilities for success with SAXS on coal, because of the great intensity, defined polarization, and wavelength tunability of the $\mathrm{X}$-rays.
Small angle neutron scattering (SANS) might be used to complement SAXS studies of coal pore structure. Since the wavelengths of the neutrons are longer than those of X-rays, SANS would have an advantage in resolving behavior of macropores (those with diameters of hundreds of $\AA$ ). The great penetrating power of neutrons, allowing the use of larger samples, might also be an advantage. The ability of neutrons to look at all elements and distinguish between them (including changes of magnitude and sign of scattering length through the use of isotopes) could be used to examine behavior of molecular species resident in the pores.

Determination of the size distribution function for coal pores by SAXS or SANS would benefit from improvement in the data analysis technique. This analysis (Chiche et al., 1965; Lin et al., 1978; Schmidt et al., 1981) is based on theories of small angle X-ray scattering from two phase (i.e. in our case, coal and voids) systems. The experiments measure the intensity $I$ as a function of scattering vector $K$. For spherical scatters (in our case, spherical voids), this intensity is related to the size distribution function $N(R)$ through the integral relationship

$$
I(K)=C \int N(R) V^{2}(R) F^{2}(K R) d^{3} R
$$

(III-B-1)

where $V(R)$ is the volume of the scatterer and F(KR) is a shape function. Thus some approximations must be made to extract $N(R)$ from this integral relationship.* The techniques used currently require assuming the shape of the pores, and this is taken as spherical. Thus one surrenders the possibility of extracting information about the shape of the pores. Since, in fact, pores are thought to be more slitlike, this is information of interest. (Information about the pore shape can, however, be obtained from electron microscopy experiments as long as the pores are reasonably large, i.e. greater than a few tens of Angstroms in mean diameter.)

Even with the assumption of spherical pore shape, both techniques currently used to extract $N(R)$ from knowledge of $I(K)$, through the relationship given by Eq. (IIIB-1), have difficulties. One technique involves

*H.R. Child of Oak Ridge National Laboratory has provided detailed information on current analysis technique to obtain $N(R)$ from small angle scattering experiments. 
numerical inversion of the $I(K)$ vs $K$ curve to get $N(R)$ vS. R. For spherical pore shape, this involves the Mellin transform equation. The numerical integrations involved require knowing $I(K)$ to large $K$ values, and are meaningful only if $I(K)$ shows Porod limiting behavior (Lin et al., 1978),, $1 / K^{4}$, for large K. The other technique currently used involves a least mean squares fit of the $I(K)$ vs $K$ data to a sum of coefficients times shape function values,

$$
I(K)=\sum_{n}^{M} A_{n}(R) F_{n}^{2}(K R) \quad(I I I-B-2)
$$

where the assumption of spherical shape gives $F_{(K R)}$. The $A(R)$ from the fittings are then the values of the size distribution function $N(R)$ at certain $R=R_{n}$, i.e. $A_{n}(R)=N\left(R_{n}\right)$. If the fit is done directly, the $N\left(R_{n}\right)$ obtained do not give reasonable behavior, because of the limited numbers of $A$ 's used and the finite range of $K$ measured. Weighting of the various terms in the expansion of Eq. (IIIB-2) and other constraints are used to "guide" the fitting procedure towards a reasonable result. Finally, with regard to quantitative characterization of the pore size distribution, we should again emphasize the desirability of doing this under dynamic conditions. That is, we want to be able to follow changes with time under realistic conditions of temperature and pressure as exist in gasification and liquefaction conditions. Recently Maylotte et al., 1981, have shown that X-ray computed tomography, aided in some circumstances by xenon acting both as a probe and contrast agent, can be used to follow changes in the average pore structure of coals and reacting coal chars in-situ.

\section{b. Study of molecular species resident in and moving through pore structure}

As already discussed, reactivity (in coal gasification and liquefaction processes and in combustion) depends to a great extent on the transport of gas and liquid (e.g., donor solvent) in the network of coal pores. This involves the interaction of the molecular species of interest with the pores (Simons and Finson, 1979). Mraw and Silbernagel, 1981, have pointed out the value of combined studies, coordinating results from measurements determining long-time, macroscopic average behavior of a fluid resident in the pore structure with results from other measurements providing a picture of motion on the molecular scale at short times ( $10^{-5}$ to $10^{-10^{2}}$ seconds). In their own study of water in coal (Mraw and Silbernagle, 1981), heat capacity measurements (Mraw and Naas-O'Rourke, 1979) have been used to give the time-averaged behavior; while nuclear magnetic resonance (NMR) (Silbernagel et al., 1979; Whittingham and Silbernagel) has been used to study dynamic effects.

Near 120K and again at room temperature, the heat capacity of water in coal pores is similar to that of bulk water; but, at intermediate temperatures where bulk water has the first-order freezing transition, there are marked changes (Mraw and Silbernagel, 1981). For low concentrations $\left(0.16 \mathrm{~g} \mathrm{H}_{2} \mathrm{O} / \mathrm{g}\right.$ coal) of water, the heat capacity (i.e. incremental difference from dry coal) gives no evidence of a phase transition; while at higher concentration ( 0.32 or $0.37 \mathrm{~g} \mathrm{H}_{2} \mathrm{O} / \mathrm{g}$ coal) a broad heat capacity maximum is observed, i.e. still no evidence of a latent heat. Mraw and Silbernagel, 1981, have analyzed this behavior with a model that suggests that approximately two monolayers of surface adsorbed water inside coal pores become "non-freezing" (or "already frozen").

Wideline measurements of the shape and width of the NMR absorption, and transient NMR measurements of the spin-lattice relaxation time $\left(\mathrm{T}_{1}\right.$ ), probe molecular motions on the $10^{-7}-10^{-8}$ second time scale. For the water-in-coal studies, use of deuterated samples, i.e. $D_{2} O$, were useful both to decrease background signal from the organic material, and to provide complementary pictures of molecular motion (because the deutron interaction with its surroundings is of a different, much more local nature, than that of a proton). This meant that $\mathrm{D}_{2} \mathrm{O}$ observations provided an opportunity to study the molecular dynamics of water molecules resident in coal pores without the interference from paramagnetic impurities that occurred for the protons in $\mathrm{H}_{2} \mathrm{O}$. Such NMR studies showed a continuous decrease in rate of the molecular motion of water in coal as the temperature is lowered, with activation energies in the range of $0.15-0.20 \mathrm{eV}$, comparable to those in bulk water.

Besides the need for innovative experimental techniques, the study of the 
behavior of molecular species resident in, or moving through, coal may also benefit from modern theoretical approaches developed in other areas of physics. To whatever degree the interconnecting pores within coal represent a random network of transport channels, statistical mechanical and probabilistic approaches may prove to be useful in the description of the mass transfer process in pores. The percolation theory (Kirkpatrick, 1973; Balian, 1979) approach, for example, could be useful here, since it has proven successful in solid state physics and other applications. It is also of interest that the effective medium theories of inhomogeneous materials developed within the context of condensed matter physics have been successfully applied to the study of the electrical (Sen et al., 1981; Mendelsohn and Cohen, 1981) and mechanical (Johnson and Sen, 1981) properties of the porous, sedimentary rocks of importance in oil and gas reservoirs. Indeed it has been shown that the Biot slow wave velocity within a fluid containing a porous medium is the same as the velocity of fourth sound in super fluid helium contained within the same medium; and the two velocities are related to the d.c. conductivity when the fluid is an electrolyte (Johnson and Sen, 1981). Monte Carlo studies offer yet another approach that may prove to be useful. These macroscopic approaches could reveal valuable, general, systematic behavior in coal transport that may otherwise be masked in studies at a microscopic level. As another example of modern theoretical approaches that might be carried over to studies of molecular species resident in the coal pore structure, the ideas of de Gennes, 1979, on interaction of confined polymer solutions with walls might be relevant.

\section{Recommendations}

- We strongly urge further work on quantitative characterization of coal pore structure, especially including the development of reliable non-intrusive physical probes such as SAXS and SANS, and with emphasis on measurement under dynamic conditions (i.e. following changes with time under realistic operating conditions of temperature and pressure.)

- We recommend emphasis on studies of interaction of resident molecular species with the pore surfaces, and of transport of such species through the pore structure. Such studies can provide information of great practical use. Research programs coordinating measurements yielding long-time average behavior with other measurements yielding short-time dynamic behavior may prove especially valuable.

- Greater attention should be paid to the possible relevance of percolation theory and other statistical approaches to the study of molecular transport processes through the coal pore structure.

\section{References}

Balian, R., 1979, Lectures in Ill-Condensed Matter, North Holland.

Chiche, P., S. Durif, and S. Pregermain, 1965, Fuel 44, 5.

Cohen, M.H., $1 \overline{981}$, to be published.

deGennes, P.G., 1979, Scaling Concepts in Polymer Physics, Cornell University Press; especially see pp. 88-97.

Grimes, W. R., 1980, "The Physical Structure of Coal", in Advances in Coal Chemistry, Vol. 1, Chapter 2, Academic Press.

Johnson, D. L. and P. N. Sen, 1981, to be published.

Kalliat, M., C. Y. Kwak, and P. W. Schmidt, 1981, in New Approaches in Coal Chemistry (B. D. Blaustein, editor), Amer. Chem. Soc. Symposium, Pittsburgh, PA, Nov., 1980, published by Amer. Chem. Soc., Washington (in press).

Kirkpatrick, S., 1973, Rev. Mod. Phys. 45, 570.

Lin, J.S., R. W. Hendrick, L. A. Harris, and C. S. Yust, 1978, J. Appl. Cryst. 11, 621.

Maylotte, D. H., R. L. St. Peters, P. G. Kosky, E. J. Lamby and C. Spiro, 1981, "Use of X-Ray Computerized Tomography in Coal Characterization", presented at the American Chemical Society Meeting, Atlanta, March, 1981.

Mendelsohn, K. and M. H. Cohen, 1981, to appear in Geophysics.

Mraw, S. C., and D. F. Naas-O'Rourke, 1979, Science 205, 901.

Mraw, S. C., and B. G. Silbernagel, 1981, pp. 332-343 in Chemistry and Physics of Coal Utilization - 1980, AIP Conference 
Proceedings No. 70 (edited by B. R. Cooper and L. Petrakis, New York.

Schelton, J. and R. W. Hendricks, 1978, J. Appl. Cryst.

Schmidt, P. W., M. Kalliat, and C. Y. Kwak, 1981, in Chemistry and Physics of Coal Utilization - 1980, AIP Conference Proceedings Series No. 70 (edited by B. R. Cooper and L. Petrakis, New York.)

Sen, P. N., C. Scala, and M. H. Cohen, 1981, Geophysics 46, 781 .

Silbernagel, $\bar{B}$. G., L. B. Ebert, R. H. Schlosberg and R. B. Long, 1981, ACS Advances in Chemistry 192, 23.

Simons, E. and J. Finson, 1979, Comb. Sci. Tech. 19, 217; 19, 227.

Taylor, S. R., K. J. Miller, and A. J.
Deurbrouck, 1981, pp. 344-356 in Chemistry and Physics of Coal Utilization -1980, AIP Conference Proceedings Series No. 70 (edited by B. R. Cooper and L. Petrakis, New York).

Walker, P. L., 1980, "Microporosity in Coal - Its Characterization and Its Implications for Coal Utilization", Invited Talk, Royal Society Meeting.

Whittingham, M. S. and B. G. Silbernagel, 1978, "NMR Techniques for Studying Ionic Diffusion", in Solid Electrolytes: General Principles, Characterization, Materials, Applications, edited by P. Hagenmuller and W. van Gool, Academic Press, New York. 


\section{iII. C. ANALYTICAL TECHNIQUES}

\section{Introduction}

This section is intended to pinpoint some selected analytical techniques which could be profitably applied to coal utilization and synthetic fuel production. A comprehensive and recent discussion of current analytical techniques may be found in the three volume work of Karr $(1978,1979)$. We have selected techniques which show the most promise for providing significant information on key problems or which offer new approaches to these problem areas. Some are presently utilized by the physics community and some are under extensive development but have not been applied to coal. The complexity of coal and its derivatives imposes unusual demands on analytical techniques.

The development of analytical techniques would be greatly assisted if properly selected and preserved reference coals were widely available to analysts and basic coal research groups. There have been only a few sample banks in the United States in recent decades where a scientist or analyst could obtain a characterized sample. Pennsylvania State University in coordination with the office of Coal Research (later the Energy Research and Development Administration and presently the Department of Energy) has had selected coal samples available. Other repositories are the National Bureau of Standards, State Geological Survey Laboratories and several private corporations (whose samples have not been available to all interested researchers), e.g. Exxon, General Electric, etc. Analytical and basic research results would be significantly more valuable if a limited number of carefully selected, characterized and stored coals were studied so that results from different studies could be directly compared.

\section{Selected problem areas}

\section{a. Elemental composition}

Of the major elements in coal, accurate (two significant figures) and direct determination of oxygen is most lacking and considered to be most important for the understanding of chemical structure. A rapid simultaneous determination of the important nitrogen and sulfur heteroatom content, as well as trace element analysis, would be important for control of processes and pollution. Neutron activation, various X-ray, electron, proton or alpha particle probes, and laser diagnostic methods appear to have some promise as analytical techniques. Many of the standard techniques (Karr, 1978,1.979) for elemental composition (e.g. ultimate analysis) will continue to find useful application.

\section{b. Chemical structure}

As indicated earlier, there is no unique chemical structure of coal. Several models have been proposed which approach this question from different and useful vantage points; e.g., treating coal as a cross-linked macromolecular gel, an amorphous semiconductor, a conglomerate organic structural unit, or a collection of micelles embedded in a plastic matrix. Different models make different assumptions concerning the carbon "backbone," and the nature and degree of linkages. Information concerning the distribution and local environments of the heteroatoms would be useful for a critical comparison with model structures. In addition, knowledge of the distributions of some of the heteroatoms ( $N$ and $S$ ) might facilitate their removal during coal processing to circumvent problems of catalytic poisoning, product instabilities, and pollution. A recent review of this subject has been given by Davidson (1980). A number of recent studies are also to be found in the German and Japanese literature (Ting, 1979; Hombach et al., 1979; Hombach, 1980; Maekawa et al., 1977). Refinements and extensions of NMR and various double resonance techniques seem to be promising approaches. Introduction of NMR-active species may prove to be a useful supplementary tool for these studies.

One of the structural parameters of interest in coal is the molecular weight distribution of the clusters between cross-links of the coal structure (Lucht and Peppas, 1981). A major difficulty in obtaining this information is the problem of breaking the cross-links and separating the fragments under conditions mild enough to permit interpreting the results as representative of the solid. A further problem is that many of the present experimental measurements (e.g. gel permeation chromatography and vapor phase osomometry) deduce number-average molecular weights, which tend to give excessive weight to low molecular 
weight fractions. Various techniques of mass spectrometry (e.g. plasma desorption and field ionization) should yield distributions of molecular weights. Interpretation of the experimental results also depends on assumptions about the shapes and structures of the fragments. Development of more direct analytical techniques would be useful in addressing this important structural problem. Small angle neutron scattering is a possibility.

\section{c. Physical structure}

The pore structure of coals affects access and egress of reactants and products, surface wetting, and bulk mechanical properties. The classic adsorption desorption measurements (Karr, 1978,1979) yield surface area estimates that depend strongly on the nature of the probing molecules and on the experimental conditions. Physical techniques, such as low angle $x$-ray and neutron scattering, can also indicate surface area and pore size distributions. Reasonable correlation between the two approaches has been observed. Further refinement of the physical techniques is desirable. (This is discussed in Section III B) It may be useful to introduce materials into the pores which would alter the scattering contrast. A more direct visualization of surface pores and -- by removal of surface layers, -- interior pores as well, may be achieved by scanning electron microscopy.

Different models of coal structure imply different mechanical properties of coal. For example, the entropy change with extension can be used to discriminate among various polymer models.

\section{d. Surface characterization}

The techniques for characterizing small area, single crystal catalytic surfaces have been well developed for studies in catalysis. (Somorjai, 1979; Goodman et al., 1980) See Section IV F for further details. Some of the same experimental techniques may also prove directly applicable to coal and coal derivatives. Among the most promising $x-r a y$ and electron probe techniques are electron microscopy, energy dispersive x-ray analysis, extended $X-r a y$ absorption fine structure (EXAFS) and its recently developed surface variation, SEXAFS. In addition, photoelectron-, Auger-, and high resolution electron energy loss spectroscopy could be useful for studying surfaces and surface layers. Further discussion of the utilization of EXAFS and SEXAFS is found in Section IV F of this report. In the case of coal, it may be useful to supplement these techniques with the use of heavy atom labelling, oxidation and reduction, or site-specific reactions. Since these techniques are inherently associated with a high vacuum environment, it will be necessary to cool the samples at least to liquid nitrogen temperature, to prevent outgassing and loss of volatiles.

\section{e. Physical and chemical transformations}

Many of the techniques mentioned above may be used to study the physical and chemical transformations of coal which take place in coal utilization and synthetic fuel production. The most commonly accepted model for coal behavior during pyrolysis is that of thermolytic bond cleavage with the production of free radicals (Petrakis and Grandy, 1981a). ESR would appear to be a natural technique for investigating such phenomena. One difficulty in applying this technique, however, is the presence of free radicals in native coal even at room temperature, whose signal masks the signal from radicals newly created during processing. ESR studies to date have shown wide variations in radical formation at elevated temperatures for different macerals and -- in the case of vitrinite -- substantial increases with temperature. These measurements can be extended to typical process temperatures and pressures, and a judgment concerning free radical, or non-free radical, involvement in liquefaction mechanisms may be possible (Petrakis and Grandy, 1981b).

At elevated temperatures bituminous coals show a plastic (mesophase) behavior. This behavior is important in the preparation of metallurgical coke. Its swelling determines the balance between increase in porosity and decrease in strength. An understanding of the physical and chemical changes in this phase is also important in other coal processes. Experimental techniques which have recently been utilized to study these structural changes are Transmission Electron Microscopy (TEM), modified to include a hot 
stage (Thompson, 1981), and $x$-ray computerized tomography during pyrolysis (Maylotte, 1981).

While the study of coal itself presents significant problems resulting from the ambiguity and non-uniformity of physical and chemical structures, one need not be overly concerned about this in the case of combustion, which is by far the most common mode of utilization. Coal may be burned fully, as in a power plant, or partially, as in certain gasifiers. Even liquefaction or gasification of coal is generally aimed at producing intermediate fuels which ultimately will be burned. Thus in every coal utilization strategy, combustion plays an important role, sometimes more than once.

Understanding flames and flame phenomena requires that we consider simultaneously chemical kinetics, gas dynamics, radiative transfer, acoustics, diffusion, evaporation and nucleation. Understanding combustion, then, is a broad task involving many specialties. All, however, share the need for development of the best possible analytical instrumentation. There is need to combine high quality measurement of parameters such as temperature, velocity, and concentration of chemical species with high spatial and possibly temporal resolution.

Recently, the clear leader in instrumentation for combustion research has been the laser. Many new investigative techniques have been developed which are based upon the utilization of laser light sources: laser induced fluorescence (Eckbreth et al., 1978; Cattolica, 1981), intracavity absorption (Brink, 1980), doppler velocimetry (Dyer and Wiotze, 1979), Rayleigh scattering (Graham et al., 1974), Raman spectroscopy (Eckbreth et al., 1978; Lapp et al., 1972), stimulated Raman spectroscopy (Eckbreth et al., 1978; Bloembergen, 1967), coherent anti-Stokes Raman spectroscopy CARS (Eckbreth et al., 1978; Harvey and Nibler, 1978; Klick et al, 1981), Mie scattering (Wyatt, 1980), particulate vaporization (Hardesty, 1980), and optogalvanic spectroscopy (Vidal, 1980). More are possible. For example, the study of turbulence may be aided by picosecond tomography (Goulard and Emmerman, 1980).

Because of the potential of laser techniques for acquiring detailed information, the resurgence of interest in optical spectroscopy of combustion species seems sure to continue. Even the basic thermodynamic properties of simple molecules and radicals participating in combustion are still poorly understood. These simple molecular systems can be studied in the laboratory by increasingly sophisticated techniques of laser s p e c t ros copy, e l e c t o n - a n d molecular-beam-spectroscopies.

In addition to laboratory studies of flames, it is possible to study separately the individual processes which make up the whole combustion process. Studies of reaction kinetics are of fundamental importance here, and various techniques such as shock tube (Lifshitz, in press), flash photolysis (Michael and Lee, 1979), fast flow reactor (Howard, 1979), and molecular beam (Sibener et al., 1980) experiments are all potentially useful for such studies.

Theoretical studies of molecules and radicals found in combustion environments have progressed to the point of being predictive. Examples can be found in the literature of state-of-the-art calculations (Harding, 1981) that correctly predict reaction products, as later confirmed by unambiguous experiments (Buss et al, 1981), in spite of preexisting, contrary, data. Furthermore, molecular calculations can give a picture of reaction pathways in detail unobtainable by direct measurements. A reasonable goal of such calculations is to understand the individual molecular processes that are important in specific combustion reactions. Presently, methane combustion in air is attracting theoretical interest (Dunning, 1981).

MHD reactors, operating at high temperatures, have their own particular needs. Not only are their materials problems severe, but even the gas phase in the MHD channel is still poorly characterized. Both experimental and theoretical techniques need improvement. A particular example of the latter, which has had some success, is the program to establish a basic understanding of the conductivity of the gas in the channel. This program has involved theory at a level as basic as electron scattering of $\mathrm{f}$ toms and molecules (Lane, 1980).

\section{Selected techniques}

\section{a. Introduction}

In choosing each of the following set of experimental techniques, we have avoided discussing a number of standard analytical techniques which are routinely used to characterize coal, coal products, catalysts, 
and catalyst supports. A note of caution should be introduced at the outset to the effect that coal, being the ill-defined substance it is, a number of studies have been carried out in the past on "...poorly selected, poorly collected, poorly prepared, poorly preserved, and poorly characterized coal samples..." (Neavel, 1979). In order to improve upon this situation, the primary recommendation of the recently concluded Basic Coal Sciences Project Report to the Gas Research Institute (Basic Coal Sciences Project, 1980) is the "establishment of a facility to collect, prepare, store, characterize and distribute samples of reference coals in strict accordance with specified protocols." A comprehensive coal characterization program to evaluate coals as process feedstocks has been initiated (Neavel, 1980) by EXXON Research and Engineering Company, Baytown, Texas. Even when standardized reference coals become available, however, it must be kept in mind that key structural parameters may not be well represented by any particular selection of reference coals, and indeed coal properties may change with time, even if the samples are stored under carefully controlled conditions. In fact, the optimum storage conditions themselves have not yet been established.

A recent development has been the application of density gradient centrifugation (Dyrkacs et al., 1981) for the separation of coal macerals. This is a much more selective method than hand sorting, but the resulting samples are still limited to mg quantities. There is continuing need to develop new (e.g. ultrasonic) techniques for physical separation of macerals.

One step which might prove important for elucidating key features of coal structure would be to develop laboratory means for simulating the natural processes that lead from biological precursor materials to coal (Adams, 1970; Teichmuller and Teichmuller, 1966; Pan, 1967; Tsukashima, 1967). If realistic synthetic model substances could be fabricated on a reasonable time scale, introduction of atomic labels and probes would be facilitated and the appropriate analytical techniques could be applied in a systematic fashion.

\section{b. Spin resonance}

Magnetic resonance studies have been among the most successful in providing structural information for whole coals, soluble fractions and liquefaction products (Karr 1978, 1979). For example, by measuring various resonance parameters the distributions of molecular and electronic environments of important atoms, such as the carbon "backbone," and the heteroatoms, S, O, and $\mathrm{N}$ can be probed, and motion of molecules in the porous structure of coal can be detected. More detailed structural information can be acquired from resonance studies of coal derived liquids and can be useful in monitoring process conditions, stability of stored liquid products, and the basic mechanisms involved in liquefaction.

Huclear Hagnetic Resonance (HHR)-The nuclei which have been studied most effectively in foal and coal derived liquids are ${ }_{H}$ and ${ }^{3} \mathrm{C}$. NMR investigations are complicated by the facts that three $3 \mathrm{f}_{2}$ coal's most abundant nuclei, ${ }^{12} \mathrm{C},{ }^{96} \mathrm{O}$, and ${ }^{32} \mathrm{~S}$ have zero nuclear dipole moments. Several other nuclei, which might be useful, namely ${ }^{14}{ }_{\mathrm{N}}, 17_{\mathrm{O}}$, and $33 \mathrm{~S}$ have non-zero nuclear quadrupole moments, and have low abundance in goal. Even for the spin $1 / 2$ nuclei, ${ }^{1} \mathrm{H}$ and ${ }^{13} \mathrm{C}$, strong homonuclear or heteronuclear dipolar interactions, chemical shift anisotropies, and long spin-lattice relaxation times make it difficult to obtain structural information from chemical-shift distributions in solid coals. Furthermore, solvent extract studies are limited by the poor solubility of coal, and the lack of knowledge as to how the extract structures are related to those of the parent coals. The multiple pulse techniques or the selective decoupling techniques enhance the spectral resolution by suppressing dipolar interactions. The residual line broadening, which is mainly due to anisotropic chemical shifts, can be further reduced by superposition of magic angle sample spinning (Lowe, 1959; Bartuska et al, 1977; Maciel et al, 1981). The cross polarization technique will be crucial for the rare nuclei NMR detection (Pines et al, 1973; Maciel et al, 1981). It will transfer polarization from proton spins to the rare nuclei spins during a time which is much shorter than the spin-lattice relaxation times of rare nuclei spins, allowing for orders of magnitude faster data acquisition. Additional improvement can be achieved by making use of the multiplex advantage of pulsed Fourier Transform (FT) 
methods, or of ch multiple fast passage (correlation NMR). Although combinations of these sophisticated techniques have been quite successful in resolving interesting spectral detail, and have yielded quantitative data, in some cases resolution of the spectra or the sensitivity of the techniques needs to be improved.

One of the most basic (hitherto very elusive) pieces of information relative to the postulated coal structural models is the carbon aromaticity of the coal. The traditional view that coal is strongly aromatic has been challenged periodically over the past ten years, but the most direct ${ }^{C}$-NMR measurements confirm this view and are in gopd agreement with indifect deductions from H-NMR. Multiple-pulse H NMR with magic angle spinning currently has a resolution just less than that required to resolve directly the aromatic and aliphatic protons.

The development of better line-narrowing techniques is a matter of high priority, and if successful, could lead to significantly better microscopic information regarding chemical structures in coal. More thorough analysis and improvements of the present techniques, perhaps in conjunction with the magic angle spinning, will make important contributions in improving the spectral resolution of solid coal. For enhancement of sensitivity 3 especially for the low abundant ${ }^{5}$ and ${ }^{3} \mathrm{C}$ nuclei, the dynamic nuclear polarization technique has promise which can be achieved through microwave saturation of the free radicals which are naturally abundant in coal samples.

NMR studies of the important heteroatom, oxygen, have been restricted primarily to hydroxyl groups. These have been determined by a variety of chemical modifications, followed by MMR analysis for the added group, e.g. using $\mathrm{H}$ or ${ }^{29} \mathrm{Si}$ to classify the $\mathrm{OH}^{\prime} \mathrm{s}$ into specific grgups following trimethylsilylation, or ${ }_{F}$ following fluorine substitution. It would also be of interest to study other oxygen functional groups, such as ether linkages, which are thought to be important in the primary liquefaction step.

If line narrowing techniques could be developed, or if isptope enriched samples could be prepared, ${ }^{O}$ NMR studies might be pqsible in the solid state. Such studies for 0 , or the more difficult nuclei, sulfur and nitrogen, could provide specific and useful structural information.

Wideline and transient NMR techniques can provide useful information about the motion of nuclei and the pore distribution and dipolar interactions with neighboring nuclei, as well as quadrupolar interactions with the local inhomogeneous electric fields, In fact, molecules labeled with ${ }^{2} \mathrm{D}$ and ${ }^{19} \mathrm{~F}$ have been used effectively (Silbernagle et al., 1980) to study the temperature dependences of motions of molecules introduced into the pore structures of whole coals. (See Section III B for discussion of NMR studies on water in coal.) When combined with ESR spin label studies, such NMR experiments can yield important information about the coal's pore structure, internal surface adsorption and diffusion characteristics for the "guest" molecules, and interactions between the molecules and chemical functionalities in the coal matrix. Similar measurements may also be carried out on the pores of the important zeolite catalysts (See Section IV F). Nuclear spin resonance has been used for some time as a simple process monitor for the quantity of moisture in coals.

Electron Spin Resonance (ESR)-- This technique has been used extensively to study the distribution of geologically stable free radicals in native coals and the pyrolytic generation of additional free radicals in whole coals, coal fractions and coal macerals (Petrakis and Grandy, 1981b; Retcofsky et al., 1979; Petrakis and Grandy, 1978; Petrakis and Grandy, 1981a). The observed variations in g-value among whole coals correlate reasonably well with the variations in atomic fraction of oxygen and sulfur, weighted according to the spin-orbit coupling constants of these heteroatoms (Retcofsky et al., 1979). This information has been compared with measured g-values for pure compounds to suggest positible model compounds related to coal (Petrakis and Grandy, 1978; Petrakis and Grandy, 1981a).

A striking result of ESR studies of macerals is the observed difference of spin concentrations by 4-5 orders of magnitude (Petrakis and Grandy, 1981a) (fusinite> vitrinite > resinite). Free radical. concentrations of all macerals, moreover, remain essentially unaffected by temperature up to 400C. At higher temperatures, those of vitrinites increase rapidly with temperature and approach typical fusinite spin concentrations (Petrakis and Grandy, 1981a). Changes in g-value with temperature for the 
various macerals provide additional indirect structural information.

Since it is thought that the initial free radical formation upon heating may be quickly followed by the formation of polymeric products, it is useful to carry out ESR experiments as a function of time. A recent pyrolysis experiment (Grandy and Petrakis, in press) along these lines, carried out on a small sample heated in a microwave cavity, showed a linear increase in spin concentration for the first 2 to 5 minutes of the experiment, followed by a decrease presumably confirming that polymerization occurs.

ESR studies of the properties of free radicals as a function of various solvent treatments appropriate for coal liquefaction indicate that residual free radical concentrations in resinite and fusinite macerals are relatively insensitive to the types of solvent, whereas high volatile bituminous vitrinites have radical concentrations which are solvent sensitive (Petrakis and Grandy, 1981a).

In view of the probable importance of free radical behavior in determining the rate of coal liquefaction, further ESR experiments on a variety of coal components and derivatives should be carried out. The availability of better separated maceral constituents (Dyrkacs et al., 1981) could be quite helpful in studying the systematics; and currently available instrumentation (Grandy and Petrakis, in press) for ESR experiments at pressures up to $2000 \mathrm{psi}$ and temperatures to $500 \mathrm{C}$ will allow measurements of free radicals during coal liquefaction. Further short scale (minutes), time-dependent experiments would also be useful.

Nuclear Quadrupole Resonance (NQR)-This technique has found limited application in coal research because of the low ganceptration and/or isotopic abundances of $97_{0},{ }^{\mathrm{N}}$, and $3 \mathrm{~S}_{\mathrm{S}}$, and because of the lack of sufficiently sophisticated equipment (Marino et al., 1980). It has become possible jn $^{\prime}$ recent years, however, to detect 0 quadrupole resonance signals in polycrystalline solids with the isotope in its natural abundance $(0.037 \%)$ using double resonance techniques (Smith, 1980).

In their review of potential applications of NQR to energy related problems, Marino et al. (1980) suggest the possibility of continuous measurement of the distribution of sulfur between organic and inorganic phases in coal, since the nuclear quadrupolar coupling interaction is very sensitive to the local environment of the probe nucleus. Better knowledge about the sulfur would help greatly in understanding the structures in coal, in dealing with the poisoning of coal process catalysts, and in dealing with emissions of sulfur oxides $\left(\mathrm{SO}_{\mathrm{X}}\right)$.

We think the possiblity of selectively introducing these quadrupolar isotopes in the coal matrix or its pores as NQR probes of the chemical and physical structures of the coal should also be explored.

Electron Nuclear Double Resonance (ENDOR) - - Potentially, this technique is capable of providing direct information about the free radical intermediates thought to play key roles in coal liquefaction mechanisms. Most previous studies (Schlick et al., 1978; Mi yagawa and Alexander, 1979; Miyagawa and Alexander, 1981; and West and Cannon, 1981) have resulted in single-line proton matrix ENDOR resonance structures which require considerable analysis to extract useful structural information. Quite different environments for the unpaired electrons have been demonstrated for the macerals vitrain, clarain and fusain from a bituminous coal, but because of the unresolved hyperfine structure, no direct conclusions could be drawn concerning the details of the structures. In contrast to this experience, ENDOR spectra on a vitrain-rich Pittsburgh coal, obtained in the absence of air, showed well-resolved hyperfine structure (Retcofsky et al., 1979) which disappeared when air was admitted. This result has since been reproduced in another laboratory (Retcofsky, 1980) and suggests that ENDOR techniques could play a leading role in the future in unraveling the details of the free radicals in coal and their role in liquefaction processes.

\section{c. Electromagnetic spectroscopies}

The most obvious feature about coal is that it is a highiy absorbing material: "black as a lump of coal." Higher rank coals are shiny, mainly as a result of the refractive index change at the surface. A considerable amount of scattering from inhomogeneities 
occurs at all wavelengths, and coals have a remarkable degree of fluorescence, especially the lower rank coals that retain a resemblance to their "humic matter" origins. These properties can form the basis for the investigation of coal and coal transformations by electromagnetic radiation.

Direct imaging or diffraction techniques, depend upon the wavelength of the radiation for the scale of the features in the material to be probed. These techniques will naturally use short wavelengths to observe structure on an atomic scale. Longer wavelength techniques may also provide such information if probes, either natural or artificial, are used to label sites in the material. As a result, the ability to identify naturally occurring labels or absorption centers in coal, or to introduce into a coal or coal-derived material artificial molecular sites or atoms that may be used to provide information about their microscopic environments, will be extremely important in analyzing the structure of coal and how it transforms under process conditions.

Reflectance and Adsorption in Ultraviolet (UV), visible and near Infrared (IR) - - Reflectance is used to identify and classify coal, both in the laboratory and in industry. Petrographic sorting and typing of macerals is done by, or assisted by, reflectance measurements (Ting, 1978; Davis, 1978). Classic studies of reflectance, and thus of the refractive index change at an air/coal or oil/coal interface, have been used to gain insight into such fundamental properties as the average size of the aromatic units in coal. The index of refraction of vitrinite is approximately 1.5 to 2.0 in the visible (van Krevelen and Schuyer, 1957). There is, however, little understanding of the basic reasons relating to the structure of the material for the functional form of the index versus wavelength. This situation is only made worse by lack of data outside of the visible region.

A firm understanding of the nature of the absorbing structures in coal would increase the usefulness of UV, visible, and near IR spectroscopy as analytical techniques in coal chemistry and coal processing. In the visible, the imaginary component of the index of refraction of vitrinite is approximately 0.02 to 0.5 (van Krevelan and Schuyer, 1957). Our understanding of absorption mechanisms in coal is disappointing, and in some spectral regions there are no published data (see Fig. 1). A major complication in acquiring such data is the presence of strong scattering; in this regard photoacoustic techniques may prove to be useful. There is a consistent pattern to optical absorption in coals -- an almost steady increase in absorption from the IR to the UV. It is unlikely that the coal coloring results from a single type of absorbing center; one expects a wide variety of absorbing groups in keeping with the complex diversity of coal structure.

Certain features may be the result of a restricted class of absorbers. It is possible to produce the UV spectrum of pyridine extracts of coal using a mixture of aromatic hydrocarbons (de Ruiter and Tschamler, 1958), but this procedure cannot be said to uniquely identify the absorbing sites in coal as aromatic groups. For example, the UV spectrum shows a feature at $265 \mathrm{~nm}$, which may be caused by aromatic ring structures (Humphreys-Owens and Gilbert, 1958), or, as has been alternatively suggested, by di-ketones (Friedel and Queiser, 1959).

The distribution of absorbing centers is not known. Thin sections of coal under the microscope have been shown to be quite colorful, and this offers the hope that locally, on a microscopic scale, absorption spectra may show more spectral detail than does a bulk sample. Spectroscopic studies of coal

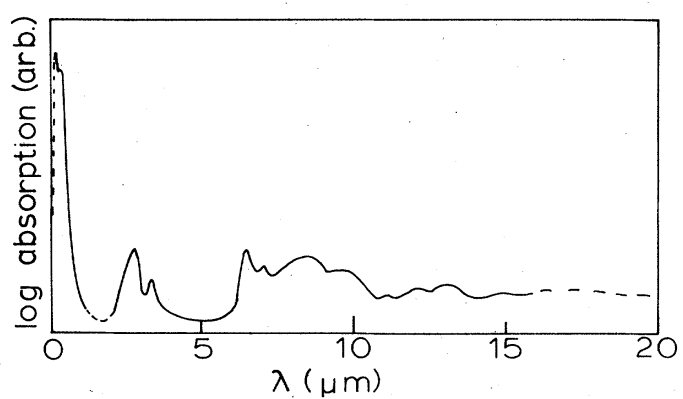

FIG. IIIC-1. Schematic of the electromagnetic absorption spectrum of an $85 \%$ carbon coal. Dashed lines indicate regions of the spectrum where there are no published data: wavelengths shorter than $0.2 \mu \mathrm{m}$, the near IR region between 0.7 and $2 \mu \mathrm{m}$, and wavelengths longer than $15 \mu \mathrm{m}$. Many of the observable absorption features have been assigned to molecular structures within the coal. Two notable features, the strong UV absorption around $0.25 \mu \mathrm{m}$ and the IR absorption near $6 \mu \mathrm{m}$ have uncertain assignments, yet they are both very characteristic of coals. 
under the microscope appear not to have been done as yet. Only recently has the increased transparency of coal and coke in the near IR been shown to be useful in microscopy (Harris and Yust, 1979). The visual appearance of coal under the microscope has long been a significant criterion for classification of various coal types, and the combination of microscopy with various forms of spectroscopy may provide important new information.

Ultimately, we want a consistent, and relatively comprehensive, picture of the nature of absorption and dispersion of electromagnetic radiation in coal. We need answers to basic questions regarding the cause for the increasing absorption of coal in the ultraviolet, the nature of the absorbing centers, the variability of absorption from coal to coal, and the role played by the absorbing centers in the other physical and chemical properties of coal.

Additional information might be gained, perhaps, by shifting attention away from the naturally occurring absorptions in coal, and focusing attention on various modified coals. It may be possible to "stain" coal with various absorbing or fluorescing compounds which highlight particular features. Also, optical studies of synthetic coal at various stages of synthesis could contribute to our understanding of the optical properties of natural coal.

Vibrational Spectroscopy: IR and Raman -- Molecular vibrations generally have associated with them wave numbers in the region from $4,000 \mathrm{~cm}^{-1}$ down to about $100 \mathrm{~cm}^{-1}$, a region customarily termed the "vibrational infrared." In contrast to other portions of the spectrum, absorption of coal in the vibrational IR exhibits a great deal of structure, with many resonances corresponding to frequencies of molecular vibrations. (Brown, 1955) Certain of these spectral features correspond to the vibrational motion of well-known chemical configurations, and estimates of the amount of aromaticity of the carbon, along with the probable nature of the bonding of heteroatoms, have been inferred from the IR data. (van Vucht et al., 1955; Retcofsky, 1977)

Interpretation of IR data is not always unambiguous, however, and is beset with both qualitative and quantitative problems. A typical problem concerns prevalence of the carbonyl ( $\mathrm{C}=0)$ configuration in the organic portion of coal. Ordinarily, the analytical organic chemist gets excellent information on this question from the intensity of absorption in the $1650 \mathrm{~cm}^{-1}$ region, characteristic of $\mathrm{C}=0$ stretch motion. In coal, there is a strong distinct absorption at $1600 \mathrm{~cm}^{-1}$ (Retcofsky, 1977), and a straightforward interpretation would be to assign this feature to a carbonyl motion (Roy, 1957; Depp and Neuwroth, 1958). However, the known presence of aromatic rings in coal complicates the interpretation by introducing another possibility: some workers have assigned this same spectral region to $\mathrm{C}-\mathrm{C}$ motions in aromatic rings (van vucht et al., 1955; Brown, 1955; and Orchin et al., 1951). Furthermore, the possible existence of extended graphitic structures in coal has led to suggestions that this absorption may be the result of donor-acceptor behavior (Elofson and Schulz, 1967), or of noncrystalline, graphitic features of the coal structure (Friedel et al., 1971). In this way absence of unique interpretations limits the ability of IR spectroscopy to answer questions of composition and structure directly.

Inherent in vibrational spectroscopy are also problems of quantitative intensity determinations. The vibrational frequencies of a particular group of atoms remain relatively constant, while their IR absorption strength varies from molecule to molecule. This makes difficult the use of IR as a quantitative indication of the amount of any particular chemical functionality present in a sample of unknown or diverse character, such as coal.

Subject to the foregoing limitations, IR can provide considerable versatility in the analysis of coal and coal derived products. A c lear advance has been made in IR instrumentation through the introduction of the Fourier Transform IR spectrometer (FTIR). This device enjoys both a multiplex and a through-put advantage. It collects data at all frequencies at all times, and utilizes a significantly larger solid angle than a comparable grating instrument. Furthermore, the instrumental resolution inherent in its optics can be made high. These advantages make FTIR well suited for the measurements of small differences in the spectra of various coals, or a single coal during processing (Solomon, 1981).

One method of circumventing some of the spectroscopic ambiguities in structural studies is by chemically modifying specific functionalities within the coal and observing the resulting shifts in the spectrum. For example, chemically attacking $C=0$ groups while 
leaving aromatic structures intact might be one way of identifying the nature of the functional groups giving the $1600 \mathrm{~cm}^{-1}$ bands in coal. Some limited work of this type has been done on coal liquids, (Fujii, 1963) but the inability of chemical reagents to penetrate the bulk coal is a problem. Some type of surface spectroscopy may provide the technique necessary to pursue this type of work; photoacoustic IR appears promising. This technique has been used to observe the aging of coal surfaces, exhibiting details not accessible with other methods (Rockley and Devlin 1980). The possibility of monitoring surface effects, as well as the capability to use bulk samples rather than finely divided powders or thin sections, makes the further development of the photoacoustic IR technique very attractive.

Raman spectroscopy, like IR spectroscopy, is capable of supplying informatign about molecular vibrations. About one in $10^{\circ}$ photons of visible light impinging upon a sample scatters inelastically. By measuring the energy loss of the scattered photon, the energy of an accompanying vibrational excitation is determined. Because the selection rules for IR and Raman differ, the two spectroscopies provide complementary information on vibrations. Raman spectroscopy of coal has been limited by the presence of a background fluorescence signal, which may obscure the Raman signal (Makovsky et al., 1971). This problem might be circumvented by working at shorter wavelength (Ziegler and Hudson, 1981). By working at shorter wavelengths, such as 200 nm, it may be possible to shift the Raman signal to wavelengths shorter than those of the fluorescence, thereby removing the interference.

Another advantage may accrue in the UV. At these short wavelengths one would be doing resonance Raman spectroscopy, since the wavelength will correspond to that of one or more electronic absorptions in coal. By investigating the dependence of the Raman signal upon wavelength, it may be possible to identify not only the Raman active vibrations, but the electronic absorbing groups participating in the Raman process. Thus it may be possible to cross-correlate features that are observed in electronic spectroscopy with those in vibrational spectroscopy. An example of this is the Raman feature corresponding to a vibration of about 1600 $\mathrm{cm}^{-1}$, which may correspond to an aromatic ring motion. If the assignment of the previously described (Davis, 1979; Schuyer and van Krevelan, 1959; de Ruiter and Tschamler, 1958; Humphreys-Owens and Gilbert, 1958; Friedel and Queiser, 1959; and Speight, 1971) $265 \mathrm{~nm} \mathrm{UV}$ absorption to aromatic structures is correct, the Raman signal at $1600 \mathrm{~cm}^{-1}$ should be enhanced as the wavelength of light used in the Raman measurement approaches $265 \mathrm{~nm}$. If, on the other hand, the interpretation of the 265 $n m$ UV absorption as a di-ketone feature is correct, ketone features in the Raman spectrum should be enhanced. This example suggests that, despite significant instrumental difficulties, Raman spectroscopy should prove useful in the analysis of coal.

Laser plune spectroscopy - Significant radiation loading can seriously damage a sample of coal. This is especially possible with laser techniques. In most cases one wishes to minimize damage by using low power levels and efficient data collection, but in some cases this damage can itself be useful. A laser can induce very rapid but controlled heating of a sample, and this may be useful in studies of desorption or pyrolysis (Hanson and Vanderborgh, 1980). At very high power loading, a plume of vaporized material is emitted from a surface that is almost instantaneously heated by a short, focused laser pulse. The ability of a laser to vaporize material in this manner gives rise to several laser plume spectroscopies. A pulse can vaporize a small sample, and the resulting plume can be investigated by optical or mass spectroscopic techniques. Since a laser plume originates from a very well defined focal region (Hanson and Vanderborgh, 1980), a laser probe may be combined with scanning microscopic techniques to give a point-by-point analysis of a material. One application is atomic analysis on a microscopic scale. This procedure already has been applied to biological samples (Sumino et al., 1980). An advantage of the laser probe over other techniques for microscopic elemental analysis, such as Secondary Ion Mass Spectroscopy (SIMS) or electron microprobe, is its ability to work under a normal atmosphere.

An alternative spectroscopic technique could be emission or resonance fluorescence analysis of the plume contents. A promising technique is the use of a tunable laser for both plume production and subsequent multiphoton ionization of the plume contents. By tuning to one particular atomic resonance, the ionization of the plume will be 
proportional to the presence of a particular element in the material (Hurst et al., 1979). It should be possible to map the microdistribution of trace elements in coal in this manner.

In addition to the atomic composition analysis by such techniques, it is possible that chemical structure information may be obtained by laser plume spectroscopies. Almost with the first availability of lasers, there has been laser pyrolysis work on coal (Sharkey et al., 1964). A high intensity laser beam gives rise to acetylene as the principal product (Hanson and Vanderborgh, 1980). This is consistent with what is known of the behavior of organic compounds in high laser fields, where $\mathrm{C}_{2}$ radicals are found as major species. At atmospheric pressures, products condense out of a plasma cloud, but in vacuum there may be few subsequent collisions after the product chemical is formed. A systematic understanding of these effects may give direct information about such features as the environments of heteroatoms. For example, in vacuum laser pyrolysis an amine nitrogen might come off principally with the carbon as $\mathrm{CN}$, or with the hydrogen, as $\mathrm{NH}$ or $\mathrm{NH}_{2}$. If, then, there are characteristic laser "cracking patterns", one may be able to assay the nearest neighbor environments of important heteroatoms.

Photoacoustic spectroscopy -Photoacoustic measurements, including the technique of photoacoustic microscopy (Quate, 1981), may be helpful in examining the structure of coal. This form of microscopy, sensitive both to the photoabsorption and to the thermal transport properties of the medium under examination, has the potential for contrast which is enhanced above that normally seen in traditional optical microscopy for petrographic analysis. The photoacoustic technique has the additional advantage of operating in air, or in other gases. Since it is inherently a scanning technique, automation should be straightforward, and development of such instrumentation could be valuable in the routine classification and characterization of coal. Another possibility is IR photoacoustic microscopy combined with optical spectroscopy, which should allow mapping of chemical functionality with a spatial resolution on the order of $10 \mu \mathrm{m}$. Shorter wavelengths would show less spectral structure, and hence provide less detailed spectroscopic information, but would allow spatial resolution on the order of $1 \mu \mathrm{m}$.
FTIR with photoacoustic detection (Royce et al., 1980; Rockley, 1979, 1980a, 1980b; and Rockley and Devlin, 1980) combines the multiplex advantage of FTIR with the ability of photoacoustic spectroscopy (PAS) to measure absorption by nearly opaque samples under realistic conditions. It is also quite sensitive to changes in surface area, could possibly give useful information about changes in pore structure and has also been used in preliminary measurements of adsorbed molecules on zeolite catalysts.

x-ray Scattering and Fluorescence -- X-ray diffraction studies have already found a place in the examination of coals (Hirsch, 1954; Pavlovic et al., 1981; Beall and Wadja, 1981; and Schmidt et al., 1981). Large angle scattering indicates the degree to which coal may be considered to be "graphitic" (Hirsch, 1954; Menster et al., 1962). Small angle x-ray scattering ( $S A X S$ ) indicates the size distribution of the pores in coal (Schmidt et al., 1981; Camier and Siemon, 1978; Beall, 1980; Lin et al., 1978) (See discussion in Section III B). Although this $x$-ray scattering work has been extensive, significant further progress can be expected. For example, by exploiting the high brightness and good collimation of synchrotron radiation, $x$-ray scattering could be carried out on a single maceral. More conventional bulk techniques should be applied to specimens consisting of separated macerals, now that the latter are available. This would permit examination of structural features ( $s u c h$ as pore size distribution) in particular macerals, perhaps providing increased understanding of the bimodal and trimodal distribution of pore sizes observed in some coals (Schmidt et al., 1981). $X-r a y s$ may be used to examine coal during reactions, as has been done in the studies of intercalation of coals by Lewis acids. (Beall, 1980)

Further insight into the meaning of surface area and porosity as defined by adsorption measurements might be obtained through small angle diffraction studies of coal with its pores filled with liquid $\mathrm{N}_{2}, \mathrm{CO}_{2}$, or other materials. One would chose material which would alter the scattering, and the effect this produces would provide information on the filling of material pores. This could be used to check assumptions made about size distributions and accessibilities of the coal pores, and could provide a cross-check on 
surface area measurements made by gas adsorption or other techniques. Contrast enhancement could also be useful in investigating the penetration of reagents into a coal sample, and the time evolution might be monitored by X-ray tomography to yield a three dimensional perspective of the diffusion processes. Such techniques have recently been exploited in X-ray computerized tomography of coal during pyrolysis (Maylotte, 1981; Kosky et al., 1981). Further aspects of SAXS for pore size determinations are discussed in Section III B.

Heavy metal labeling of functional sites, either on surfaces or in pores, could provide significant information as to the number and location of these sites in the material. More importantly, labeling would provide a measure of the radial correlation function for similar and different functionalities. For example, barium loading of acid sites, such as carbonate, would find almost immediate applicability; other labels should be developed and investigated.

These "contrast enhancement" techniques of filling coal pores with a fluid, or tagging functional sites, should also find use in neutron scattering. One could vary the scattering contrast even for one molecular substance by changing the isotopic composition.

In addition to diffraction studies using penetrating $x$-rays and neutrons, one should investigate the utility of using $x-r a y$ fluorescence (Botto et al., 1980; Prather et al., 1979) and neutron activation (Volborth, 1979a) for chemical element determination. An advantage of both techniques is the capability of determining elements in coal or coal products on-line in a process stream. Direct, quantitative determination of oxygen (which is otherwise found by differences) should be improved and made routine (Vorborth, 1979a). Neutron activation shows promise as a solution to this problem.

An intriguing extension of $x-r$ ay techniques is the recent observation of electronic Raman effects in the $X-r a y$ scattering from graphite. (Koumelis and Londos, 1980) Although the utility of the technique remains to be demonstrated for coal, its potential should be carefully examined.

Hos sbauer spectroscopy - one application of the Mossbauer effect, the determination of iron and the characterization of its chemical environment, is now a useful method in coal research, and shows promise as a standard method of assaying pyrite in coal (Huggins and Huffman, 1978, 1979; Levinson, 1981). In a recent study, a radioactive pfecursor ( $\left.{ }^{57} \mathrm{Co}\right)$ of a Mossbauer-active nucleus ( ${ }^{57} \mathrm{Fe}$ ) has been impregnated into a catalytic material to give information about its local chemical environment. (Clausen et al., 1979) There is little to prevent an extension of these techniques to the study of coal itself, using appropriate Mossbauer nuclei. Once incorporated in the coal, these nuclei may serve the additional purpose of providing information during chemical and physical changes taking place in the coal. From chemical shifts, one can now reliably determine if a particular Mossbauer nucleus is in an oxidized or reduced chemical environment, although determination of the identities of its chemical neighbors is not usually possible. Such information is quite useful, especially when one is interested in identifying changes which may occur during chemical or physical processing. Efforts should be made to exploit Mossbauer labels in coal, both naturally occurring and artificially introduced. Some further discussion of applications of Mossbauer spectroscopy to coal research is given in Section IV F.

\section{d. Electron probe and electron spectroscopy}

Electron probe and various electron spectroscopic techniques are well suited for the characterization of physical and chemical structure and composition of surfaces and surface layers. The thickness of the layer amenable to investigation ( $\sim 3$ mean free paths) depends on the nature and energy of the probing and detected particles and on the angle between the particle's trajectory and the specimen surface. (e.g. X-rays penetrate to about $1 \mu \mathrm{m}$, while low energy electrons penetrate to only a few Angstroms.) The limitation of the techniques to surface layers may seem to be a serious handicap. One should bear in mind, however, that the reactions of solid coal particles with various agents occur at the surface, and depend critically on the chemical and physical environment there. Similar considerations apply to coal products and to container materials (corrosion and degradation processes - - See Section IV D). Bulk information can also be obtained by these techniques in some cases (beyond a few atomic 
layers in the sample, properties observed are those of the bulk material) either directly, or by successive removal of surface layers.

Because of the microscopically heterogeneous structure of coal, the electron probe techniques, with their high spatial resolution, may prove to be ideal tools of investigation. These techniques require a vacuum environment and are best utilized in combination to complement each other. They are not easily adaptable for on-line quality control applications. A very important developing use of these methods is in the area of model catalytic studies (Somorjai, 1979; Goodman et al., 1980) and the determination of surface layer composition and structure (Somor jai and van Hove, 1979). (See Section IV F.) The critical questions here concern the way one should proceed from idealized laboratory samples to coal, coal products and components of coal conversion systems. The modification of conventional high vacuum apparatus to allow temperature control of the sample, the introduction of high pressure reagents, and subsequent return to high-vacuum conditions, already has made possible studies of the progess of surface reactions and changes in surface layer structure and composition (Somor jai, 1979; Goodman et al., 1980).

Electron Hicroscopy - - Electron microscopy utilizes a highly focused electron beam and in general offers excellent lateral resolution ( $100 \mathrm{~A}$ or better) and large depth of field (order of microns).

Transmission Electron Microscopy (TEM) is capable of a few Angstrom resolution but requires specially prepared samples. It has been utilized to study surface structure of coal and has yielded pore size distribution in good agreement with low-angle X-ray scattering results (Harris and Yust, 1976; Harris et al., 1977). Strehlow et al. (1978) investigated the relationship between mineral content and certain maceral types. Monitoring surface transformation with this technique can also yield valuable information; e.g. Thompson utilized a hot stage TEM and observed the development of some mesophase structure on coal at elevated temperatures (Thompson, 1981). A similar technique has been used to study the catalytic gasification of graphite (Baker, 1981).

In Scanning Electron Microscopy (SEM), the secondary back-scattered electrons are detected. This method can accept a broad range of samples and yields a picture of the surface with typical magnifications of 50 to 100,000. SEM has been extensively used (more than 10,000 instruments are operating around the world today) for studies of biological systems, minerals, microcircuits etc. Shinn and Vermealen (1979) applied SEM to coal before and after leaching with organic solvent and found it very useful in monitoring the opening up of the pore structure.

Coal ash is comprised of micrometer sized aluminosilicate hollow glass spheres. These hollows are filled with smaller spheres consisting of heavy metals and toxic elements. SEM (and TEM) has proved to be useful for determining the specific form of these deposits and for the better understanding of the accessability of the metals to leaching (Gibbon, 1979). SEM can also be valuable for the characterization of coal particles (Moza et al., 1979) and for petrographic analysis of coal macerals and inorganic constitutents (Stanton and Finkelman, 1979). Some caution has to be exercised in these studies to avoid damage to the specimen by the highly focused electron beam.

In combination with SEM one can also monitor the back-scattered primary electrons, Auger electrons or the X-ray signals. More detailed discussion of these methods is given below under the appropriate headings.

Photoelectron Spectroscopy Photoelectron Spectroscopy utilizing UV (UPS) or X-ray (XPS) photons has found wide application in the study of solid surfaces (Siegbahn et al., 1967; Carlson, 1975; Brundle and Baker, 1979a; Karr, 1978) and it can also be applied to liquids and gases. (XPS originally was called ESCA: Electron Spectroscopy for Chemical Analysis.) With this method, the energy (and sometimes angular) distribution of photoionization electrons is determined and correlated with the characteristic energies of atomic species and their environmental influence. One can obtain information on the atomic composition of the sample, and from the chemical shift, on the atomic environment. The method is applicable to all elements heavier than He. The resolution is limited by the energy width of the light source, the natural line widths of the levels involved and the electron energy analyzer. Typically line widths range from a few tenths of an eV to a few eV. The utilization of synchrotron radiation in recent 
years has made possible significant improvements in line width, in signal to noise conditions, and in scanning over a wide wavelength range.

UPS provides sensitivity to the valence, the conduction and the first energetically deeper lying bound atomic electrons. Photelectron spectroscopy of the outer shells (PESOS) has been mainly used in studying the molecular orbitals of free or adsorbed molecules. For solids and clean solid surfaces, molecular orbitals usually do not exist and the interest lies in the study of band structures. The method is useful in studying molecules adsorbed on surfaces, but has not been developed for analytical purposes. The importance of angle resolved ultraviolet photoelectron spectroscopy (ARUPS) is further discussed in Section IV F.

XPS allows deep atomic core level ionization. Photoelectron Spectroscopy of the Inner Shells (PESIS) is basically the study of atomic orbitals. The shift in the binding energies of the core electrons as a function of chemical environment can be considered as a perturbation on the atomic system. The ionization energies are characteristic of the atomic composition of the sample and the shifts in these energies indicate the nature of the chemical environment. Typical chemical shifts for core levels are of the order of a few eV. PESIS can be utilized for qualitative and quantitative analysis and for diagnostic purposes. It can identify very small amounts of material on surfaces (on the order of $10^{-2}$ monolayer). In quantitative analysis, an accuracy of about $10 \%$ should be possible. The intensity ratio of widely separated peaks in the electron energy of the same element gives an indication of the homogeneity of the atomic distribution within the sampled region (Brundle and Baker, 1979b). (Electrons of different energy sample different depths.) The technique has found wide utilization in connection with catalysts, polymers, fibers, fine particles and microelectronics. An important feature of XPS is that catalysts can be examined just before and just after reactions similar to those which take place in plant reactors. This allows correlation to be made between súf face information data and catalyst performance. However, it must be recognized that species weakly adsorbed on the surface would desorb during the XPS measurement.

Frost et al. (1974) investigated the applicability of XPS to coal analysis.
Carbon, and total sulphur determinations using XPS were found to be in good agreement with chemical analysis, but no correlation was found for oxygen. Attempts to utilize XPS to separately identify organic and inorganic sulfur in coal were not successful. XPS showed two well separated sulfur $2 p$ peaks at 163 and $169 \mathrm{eV}$, corresponding to iron sulfides and iron sulfate, respectively, but no separate $2 p$ line was found for organic sulfur. This peak would be expected in the 163-165 eV region (corresponding to thiol-, thioether, disulfide- or thiophene-type structures). It is likely, therefore, that the organic sulfur peak is overlapped by the pyrite peak.

Extension of XPS investigations to $\mathrm{C}, \mathrm{N}$, $O$, and $S I s$ and to trace metal core shells and the study of satellite structures (associated with simultaneous two and three electron excitations) may prove valuable in characterizing the chemical structure of coal. The XPS spectrum changes drastically with $X-r a y$ wavelength. This effect could be exploited to emphasize (deemphasize) certain structures for the purpose of simplifying the spectrum.

\section{Auger Electron Spectroscopy (AES)}

-- In Auger spectroscopy, the impinging electron beam (or X-ray beam) causes an atomic core level ionization. The resulting hole state may decay to a lower energy state through an electronic rearrangement accompanied by the ejection of a low-energy electron and creation of a doubly ionized state. The ejected Auger electron will have a kinetic energy which is characteristic of the parent atom (Davis et al., 1976). Since low energy electrons can travel only a few Angstroms without losing further energy and becoming trapped, the energy and to some extent the shape of Auger features can be used to identify unambiguously the composition of the solid surface. Quantitative analysis may be accomplished by comparing peak heights obtained from unknown specimens with those of pure elemental standards. This technique has a sensitivity in the range of 0.02 to 0.2 atom percent and is applicable to all elements heavier than He. Utilization of this technique in combination with SEM as the primary electron source (SAM: Scanning Auger Microscopy) is widespread. The utilization of the combined techniques for spectroscopic purposes (Scanning Auger Electron Spectroscopy: SAES) is characterized by high lateral resolution and surface sensitivity, 
which is surpassed only by Field Ion Microscopy. In Auger microanalysis (100 $\mathrm{nm}_{4}$ elȩctron beam size) a sample 6 volume of $10^{4}$ $\mathrm{nm}^{3}$, corresponding to about $10^{6}$ atoms, can be studied. Further developments are needed, however, to make this technique applicable to quantitative analysis (Van Oostrom, 1979; Goldstein and Yakowitz, 1975), and the danger exists of locally pyrolyzing the coal by the electron beam.

Energy (or Wavelength) Dispersive X-ray Spectroscopy -- The decay of inner core hole states may also occur via X-ray emission. The $\mathrm{X}$-ray spectrum is characteristic of the atomic composition and is influenced by the atomic environment similarly to the Auger electron spectrum. X-rays, however, have larger mean free paths and, therefore, sample a deeper layer than low-energy electrons.

Very frequently the electron beam is sharply focused and can be scanned over the surface of the sample (SEM). With this method, chemical analysis of a few tenths of a cubic micron volume can be carpied ouf with a sensitivity of about 1 in $10^{5}\left(10^{-} 6^{6} \mathrm{gram}\right.$ material). These electron probe microanalytical techniques have been developed for quantitative analysis of elements heavier than $\mathrm{Na}$ (Goldstein and Yakowitz, 1975 ; Anderson, 1973; Scanning Electron Microscopy, published annually). Their application in coal research is discussed by Raymond and Gooley. (1980). In another variation, the sample is scanned with the electron beam, and the $\mathrm{X}$-ray signal at a fixed wavelength is monitored. If this wavelength is characteristic of some element, the distribution of this element over the sample surface can be displayed with submicron lateral resolution.

The application of electron microprobe $\alpha$ X-ray detection techniques for the quantitative determination of organic sulfur in coal on a maceral level proved successful (Raymond and Gooley, 1978; Greer, 1979). These procedures were also used to determine inorganic and elemental sulfur. The active volume excited by the $17 \mathrm{keV}$ electron beam extended to a depth of about 3 to $4 \mathrm{~m}$ below the surface and had a width of about $1 \mathrm{~m}$. The determination of trace elements in coal by SEM microprobe techniques was also found feasible (Finkelman, 1978; Gluskoter et al., 1977).

SEM with automatic image analysis was reported very recently (Huggins et al., 1980) for quantitative mineralogical description of coal. With this method,the SEM located mineral or maceral media and then centered the electron beam on the particle automatically. An energy-dispersive $X-r a y$ spectrum covering $\mathrm{Na}, \mathrm{Mg}, \mathrm{Al}, \mathrm{Si}, \mathrm{P}, \mathrm{S}, \mathrm{Cl}, \mathrm{K}, \mathrm{Ca}, \mathrm{Ti}$ and $\mathrm{Fe}$ was accumulated in about 2 seconds.

Quantitative analysis by electron microprobe techniques compares the $\mathrm{X}$-ray intensity produced by the sample to that produced by a standard of known composition. The $X-r a y$ intensity is influenced by $X-r a y$ absorption, fluorescence and atomic number effects, and thus by the composition of the sample. The critical question here, therefore, is preparation of the standard. Further development in this area is needed before accurate analysis can be carried out on a routine basis.

Extended $x$-ray absorption Fine Structure -- EXAFS studies go back to the 1920 's. With the availability of synchrotron radiation, advances in quantum mechanical theories, and developments in signal monitoring and data analysis, this technique has developed in recent years into a powerful tool for chemical and structure analysis (Hodgson and Doniach, 1978). The extended $X-r a y$ fine structure appears as a modulation of X-ray absorption above the absorption edges. The modulation arises because the final state of the photoelectron is perturbed by the surrounding atoms. The X-ray absorption can be measured directly, or by means of monitoring X-ray fluorescence, partial photoelectron yield, total photoelectron yield and/or Auger electron yield as a function of X-ray energy. These measurements yield information about the environment of a particular element in a sample, the distance between this and neighboring elements, and the identity of these elements. This information can be obtained for trace elements, both in the bulk and on the surface, and the atoms are not required to form a periodic array. The technique is equally applicable to gases, liquids and solids. The average distance between the absorbing atom and its near atomic neighbor can be determined to an accuracy of a few hundredths of an angstrom. The method has been successfully applied to the study of supported catalysts (Sinfelt and Via, 1978) (also see Section IV F) and to biological systems (Cramer et al., 1978). Application 
was extended recently to the soft $X$-ray region where $\mathrm{K}$-edges for the important second and third row elements fall. A method developed at the Unversity of Delaware (Chem. and Eng. News, 1980) utilizes a rotating anode generator as its X-ray source and can produce EXAFS spectra similar to those produced by synchrotron sources, but with considerably less investment.

For macromolecular systems like coal, which have no crystal structure, EXAFS can identify the coordination environment of selected atomic species (heteroatoms, metal catalysts and trace elements) (Wong et al., 1981; Maylotte et al, 1981) and follow their changes as the decomposition of the sample proceeds.

$X-r a y$ absorption by a monolayer on a surface is so small that it will not show in an X-ray transmission experiment. However, recently a new technique, SEXAFS, was developed to study surfaces by measuring the modulated intensity of absorbate core Auger emission lines as a function of photon energy (Lee, 1976; Citrin et al., 1977). (Partial or total photoelectron yield can also be monitored). The underlying principle is that the Auger or secondary electron emission is directly proportional to the photoabsorption cross section, which is modulated because of interference between iutgoing and back scattered core photoelectron waves. An electron energy resolution of about $2 \mathrm{eV}$ is in general sufficient to discriminate against background electrons. Furthermore, the monitoring of low-energy electrons rather than $\mathrm{X}$-ray fluorescence, greatly reduces the background signal, since these electrons originate only a few angstroms inside the surface. The sensitivity of this method is about $10^{6}$ times higher than typical EXAFS and statistically meaning ful data can be obtained from as few as $10^{3}$ absorbers in the $X-r a y$ beam.

The SEXAFS method was applied recently to the study of oxygen adsorbed on aluminum surfaces by using monochromatized synchrotron radiation and partial electron yield spectroscopy as the detection technique (Stohr et al., 1978).

The SEXAFS technique should be useful in monitoring both simplified model catalysts and complex dispersed catalysts. Refinements and application of this method to coal catalysts seem feasible. Questions such as whether the metallic elements are combined as homogeneous alloys or in a more complicated binding arrangement with the substrate can be answered. The chemical functionality of surface $0, N, S$ could perhaps be obtained from SEXAFS studies.

\section{High-Resolution Electron} Energy-Loss Spectroscopy (HREELS) Electrons impinging on surfaces can lose part of their kinetic energy due to various processes which could include excitation of surface phonons, plasma waves and electronic surface transitions (Ibach, 1977). The interest here lies, however, in the excitation of the vibrational modes of atoms and molecules adsorbed on the surface. The energies associated with these modes fall in the 50 to $300 \mathrm{meV}$ range and are readily detectable with high resolution ( $210 \mathrm{meV}$ ) electron impact spectrometers. The electrons sample only a few angstrom layer as opposed to IR photons, which penetrate a depth of the order of a few microns and thus give bulk information. Adsorbed species with chemical bonds perpendicular to the surface are more easily detectable than those with bonds which are parallel to the surface. The sensitivity of this technique is of the order of 0.1 monolayer. Chemical bonds characteristic of the adsorbed molecule and of the binding between adsorbed atomic and molecular species are readily identified and can serve as a monitor on the nature of the adsorbed species. An important aspect of this technique is its ability to detect hydrogen, which is not readily detected by other techniques. This unique capability makes HREELS a very important tool in studying the surface chemistry of hydrogen, hydrocarbons and other hydrogen containing molecules. For example, it is easily recognized whether a molecular adsorption proceeds through dissociation or not. One can also gain knowledge of the binding potential and the affect of coadsorption. As in the case with other surface tools, this method is also best utilized in combination with LEED, Auger etc. methods.

In recent years, a number of HREELS studies have been carried out on $\mathrm{H}_{2}$, $\mathrm{CO}, \mathrm{O}_{2}$ and hydrocarbons absorbed on metaf surfaces (Froitzheim, 1977; Thiel et al., 1979; Hamilton et al., 1981). The method yields valuable insight into the mechanisms of catalytic reactions. Combination with SEM would enable one to investigate small regions of a sample. 
The application of HREELS is just developing. The question of selection rules (the relaxation of which is a major advantage in electron impact spectroscopy as compared to optical spectroscopy in the case of gaseous species) has not yet been settled and fully investigated for solids. Neither have the angular dependence, resonances and the utilization of spin polarized electrons been extensively exploited in connection with surface adsorbed species. One would certainly expect important developments in this area which should contribute to our understanding of the nature of chemisorption.

\section{e. Other techniques}

Hass spectronetry will continue to be a significant tool in the analysis of coal and coal derived liquids (Carter et al., 1979; Guidoboni, 1979; Ondov et al., 1975). Important techniques such as plasma desorption and field desorption (St. John et al., 1978), field ionization and chemical ionization should complement ordinary spark and electron beam ionization. A recent development in mass spectrometry is the use of laser ionization. A comprehensive review of this technique, as applied to solids, is given by Conzemius and Cappellen, including a direct application to coal (Conzemius and Capellen, 1980). In much of the work with this technique there has been the problem of a basic lack of understanding of the processes occuring in the ion-forming laser plasma. Recent rapid progress makes it appear likely that laser ionization will be a standard option on mass spectrometers in the next five years. Laser intensities high enough to ionize the sample are also high enough to lead to significant cracking of hydrocarbons, as evidenced by the large number of $C_{2}$ species formed with straight laser ionization. For milder ionization conditions, when study of the higher mass region of the spectrum with less cracking is desired, a combination of laser desorption with other methods of ionization shows promise. Recent work on laser pyrolysis shows that it has potential for nitrogen and sulfur determination (Hanson and Vanderborgh, 1980).

Additional advantages accrue with the ability to probe individual macerals in a sample. SIMS is well suited for this role (Wittmaack, 1979).

Crossed atomic and nolecular beam studies are capable of revealing details of chemical reactions, and are very important methods for understanding the mechanism of a given reaction. There are several problems, however, in trying to apply these methods to coal gasification and synthetic fuel production schemes. The beam-beam technique is applicable to gas phase processes, while in coal gasification and liquefaction, it is heterogeneous catalysis processes, not readily amenable to study by this technique, that are most important. The practical usefulness of beam experiments in these studies is therefore uncertain. Gas phase reactions and flames associated with coal utilization involve a large number of mutually interacting components and very complex kinetic schemes. Beam/beam studies are best suited for the detailed understanding of specific reactions, from which the complex kinetic scheme can be built up.

Ion beams - Analytical techniques based upon the use of accelerated charged particles to induce prompt $\gamma$ radiation, elastic scattering, and $\mathrm{x}$-ray radiation have proved useful for elemental analyses, including spatial surface distributions and depth profiling (Pierce, 1974; Volbroth, 1979b; Prather et al., 1979; Buckle and Grant, 1981). Prompt radiation is most useful for the lighter elements, and the technique has been used for analytical determinations of carbon, nitrogen, oxygen, and sulfur. Proton-Induced X-ray Emission (PIXE) has been used with somewhat better sensitivity than the corresponding electron-induced $x$-radiation for trace element analysis (Buckle and Grant, 1981). In addition, the incident protons can be focused to beams a few microns in diameter and brought through a window to perform microprobe analyses with the specimen at atmospheric pressure, something not possible with electron microprobes. This feature could be useful in studying elemental variations over the surface of whole coal, as well as changes during mild process conditions.

Elastic scattering of alpha particles has been most useful in the thickness determination of films of heavy elements present on lighter substrates. The technique is complementary to X-ray work, since the very low atomic mass species produce $\mathrm{X}$-rays which are too soft for convenient measurement, while alpha backscattering shows worsening resolution for higher atomic masses. The surface composition of species such as $C, 0$, $S, F e$ are readily resolved (Patterson et al., 1966). 
Heutrons are useful for activating trace elements and heteroatoms of coal and coal ash. Thermal neutrons from reactors (Steines, 1979) and fast neutrons generated by deuterons accelerated onto metallic targets (Larsen and Kovac, 1978) each have specific advantages for different elements. Fast neutrons, especially, can provide chemical composition information for the important heteroatoms oxygen and nitrogen. Neutron activation techniques also have important applications in process monitoring and control (see Section IV B).

Small angle neutron scattering, especially if combined with addition of contrast enhancement fluids, may provide useful information regarding the size distributions of coal pores. As in the case of $x$-ray diffraction, there may be merit in treating the average structure of coal as being amorphous, and using diffraction techniques to deduce positional correlation functions.

Preliminary small angle neutron scattering experiments have been carried out (Maxwell et al., in press) on coal powders and solutions in an attempt to use this technique for the determination of average molecular weight.

Elastic property measurements in coal are particularly difficult because of the heterogeneous character of the material. Nevertheless, those data which have been collected have interesting implications. For example, Larsen and Kovac (1978) have suggested that the time-dependent response of bituminous coals to a constant stress shows that they are cross-linked, non-planar molecules. This is a sufficiently important point in discriminating among proposed average molecular structures of coal that further studies of this aspect of the elastic properties should be encouraged.

An ultrasonic experiment (van Krevelan et al., 1959) obtained velocities of sound for various coals, from which carbon aromaticities were deduced. The ultrasonic values for this parameter turn out to be in rather good agreement with more direct determinations by the highly sophisticated resonance methods described in Part $3 \mathrm{~b}$, of this Section. This correlation may also be worthy of further investigation.

A more recent application of ultrasonics is that of ultrasonic microscopy of coal surfaces, (Quate, 1981) with a spatial resolution of $0.3 \mathrm{~m}$. This technique could be useful in petrography, the optical microscopy version of which depends on variations among macerals in the small ( $1 \%$ ) optical reflectivity (Ting, 1979). By comparison, the acoustic reflectance is $50 \%$, and the waves also sample the subsurface of the coal which is inaccessible to the visible light waves. Additional applications of ultrasonic techniques will be discussed in Sections IV $B$ and IV F.

\section{Recommendations}

\section{Samples}

- A sample bank of carefully selected, characterized and stored coal specimens should be established where individuals or groups could obtain representative samples for analysis or research purposes.

- More extensive use should be made of labelling techniques in connection with resonance experiments, $x-r a y$ and neutron scattering.

- Further study should be carried out to explore the possibilities of preparing small samples of synthetic coal.

- Improved petrographic and chemical characterization methods should be developed. Acoustic and photoacoustic microscopy techniques seem promising, as do $x$-ray tomography and EXAFS studies.

\section{Techniques}

NMR - - Better NMR line-narrowing capability should be developed, efforts should be made to perform resonance (NMR or $\mathrm{NQR}$ ) measurements on oxygen, nitrogen and sulfur nuclei and attempts should be made to obtain molecular structural information pertinent to linkages which play a role in the primary liquefaction step. NMR techniques applied to zeolite catalysts are promising and should be extended.

ESR and ENDOR studies have important potential for determining the influence of free radical behavior on the rate of coal liquefaction. Further studies should be carried out under process conditions, and serious attempts should also be made to carry out ENDOR studies at elevated temperatures and pressures.

Optical Properties Measurements should be made of the complex index of 


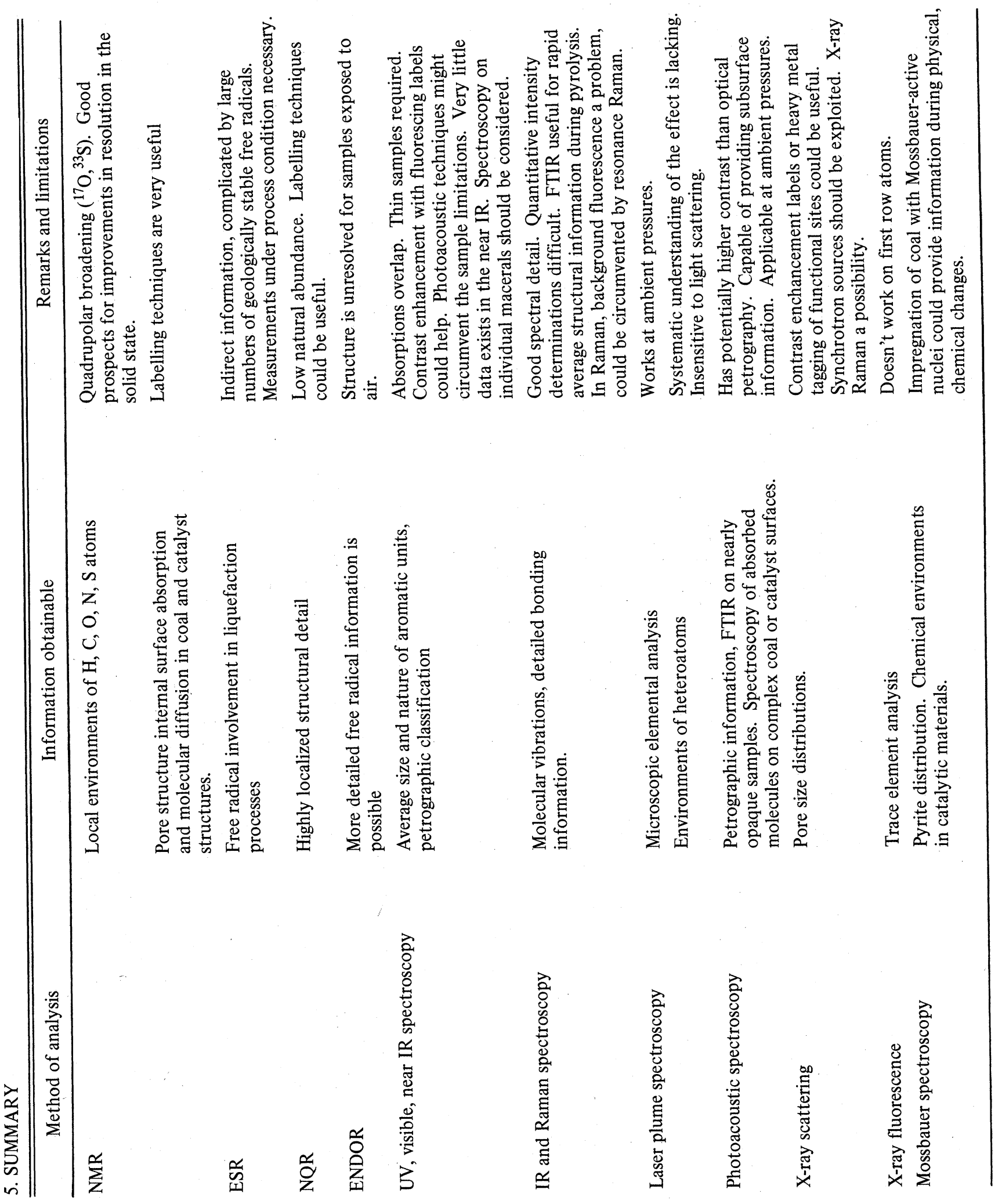




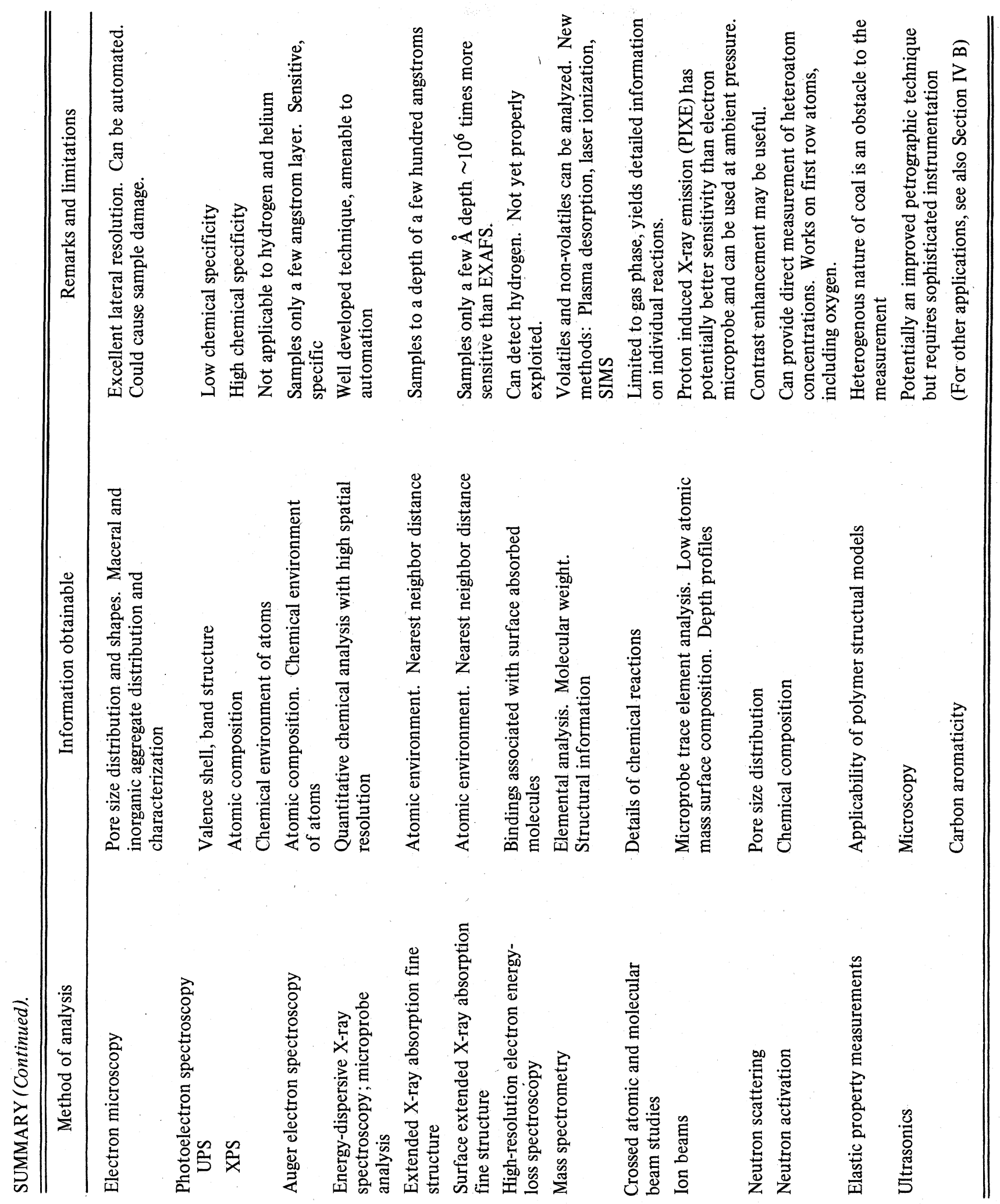

Rev. Mod. Phys., Vol. 53, No. 4, Part II, October 1981 
refraction of coal over as wide a frequency range as possible, and the results used to test features of the various average structural models proposed for coal. Microscopic spectroscopy could be especially useful in identifying structural differences among macerals in the same coal specimen. Vibrational Spectroscopy Particular emphasis should be placed on vibrational spectroscopy, both because of its sensitivity to molecular bonding and because of its potential for monitoring subtle changes which take place during Iiquefaction. Photoacoustic FTIR and resonance Raman studies could be fruitful new techniques applied to coal and catalysts.

Laser Plume Spectroscopy should be explored for its potential for providing local molecular information in addition to its established capability for microscopic elemental analysis.

Synchrotron Radiation sources should be exploited for extended $x-r a y$ scattering experiments, and contrast enchancement additive substances should be used in conjunction with both $\mathrm{x}-\mathrm{ray}$ and neutron diffraction to study the details of the pore structures of coals.

Electron Microscopy should be utilized for the determination of particle and pore size distributions and shapes. Development of automated procedures for routine measurements would be very useful.

Electron Spectroscopy Various electron probe and electron spectroscopic techniques should be pursued in a complementary fashion for characterizing surface structure and composition of coal, catalysts and container materials. The quantitative techniques for $N, 0, S$ and trace element determinations should be refined. SEM combined with energy-dispersive $\mathrm{X}$-ray analysis seems to be very promising for fast, automated, quantitative elemental analysis of mineral and maceral segments in coal. Quantitative analysis by electron microprobe techniques relies on comparison with standards. Research is required on questions concerning the preparation of standards. SEM, combined with Auger Electron Spectroscopy, SEXAFS, and HREELS are excellent methods and should be further developed for the study of surface adsorbed species. Catalysis The electron probe and electron spectroscopic techniques should be utilized in catalytic studies. A useful approach to such studies is the introduction of a high-pressure and high-temperature reaction chamber into a conventional high vacuum electron probe diagnostic system.

Neutron Activation analysis should be more widely applied for concentration determinations of first row atoms. The technique should be made generally available to workers in the field, and coal specimens should be routinely characterized for oxygen content.

\section{References}

Adams, D.C., 1970, Exothermal Metamorphosis of Coal Precursors, Ph.D. dissertation, University of Utah.

Anderson, C. A., 1973, ed. Microprobe Analysis John Wiley and Sons, New York.

Baker, R. T. K., 1981, Bull. Am. Phys. Soc. $26,200$.

Bartuska, V. J., G. E. Maciel, J. Shaefer, and E. O. Stejskal, 1977, Fuel 56, 354.

Basic Coal Sciences Project Advisory Committee Report, 1980, Prepared by Science Applications, Inc. for the Gas Research Institute, July, 1980.

Beall, H, 1980, Fuel 59, 140.

Beall, H., and R. J. Wadja, 1981, in Chemistry and Physics of Coal Utilzation -1980 (APS, Morgantown) (edited by B. R. Cooper and L. Petrakis, New York), AIP Conference Proceedings No. 70.

Bloembergen, N., 1967, Am. J. Phys. 35, 989. Botto, R. I., B. H. White and J. H. Karchmer, 1980, Fuel 59, 157.

Brink, G. O., 1980, Opt. Comm. 32, 123.

Brown, J.K., 1955, J.Chem. Soc. (London) $744,752$.

Brundle, C. R. and A. D. Baker, 1979a, eds. Electron Spectroscopy: Theory, Techniques and Application, Vol. 3, Acad. Press, New York.

Brundle, C. R. and A. D. Baker, 1979b, eds. Electron Spectroscopy: Theory, Techniques and Application, Vol. 3, Acad. Press, New York, p. 309.

Buckle, D. C., and G. C. Grant, 1981, in Chemistry and Physics of Coal Utilization - 1980 (APS, Morgantown) (edited by B. R. Cooper and L. Petrakis, New York), AIP 
Conference Proceedings No. 70.

Buss, R. J. R. J. Baseman, P. Casavecchia, and Y. T. Lee, 1981, to be published.

Camier, R. J., and S. R. Siemon, 1978, Fuel $57,508$.

Carison, T. A., 1975, Photoelectron and Auger Spectroscopy, Plenum Press, New York.

Carter, J. A., and D. L. Donohue, J. C. Franklin, and R. L. Walker, 1978, 1979, in Analytical Methods for Coal and Coal Products, ed. Clarence Karr, Jr. ; Vols. 1, 2, (1978), vol. 3 (1979).

Cattolica, R., 1981, Appl. Optics 20, 1156.

Chem. and Eng. News, July 14, 1980, p. 27.

Citrin, P. H., and P. Eisenberger and R. C. Jeweitt, 1977, J. Vac. Tech. 15, 449.

Clausen, B. S., and S. Morup and T. Topsoe, 1979, Surface Sci. 82, L 589.

Conzemius, R. J. and Capellen, 1980, Int. J. Mass. Spect. Ion Phys. 34197.

Cramer, S. P., and K. O. Hodgson, W. O. Gillam and L. E. Mortenson, 1978, J. A. Chem. Soc. 100, 3398.

Davidson, Robert M., 1980, "Molecular Structure of Coal," Report No. ICTIS/TR08, IEA Coal Research, London.

Davis, A., 1976, in Analytical Methods for Coal and Coal Products, ed. Clarence Karr, Jr., Vol. 1, pp. 27-83.

Davis, L. E., N. C. McDonald, P. W. Palmberg, G. E. Riach and R. E. Weber, 1976, Handbook for Auger Electron Spectroscopy, Phys. El. Ind. Inc.

Depp, E. A., and M. B. Neuworth, 1958, Brennst.-Chem. 39, S90.

de Ruiter, E., and H. Tschamler, 1958, Brennst-Chem. 39, 362.

Dunning, T. H., $1 \overline{981}$, private communication.

Dyer, T. M., and P. O. Wiotze, 1979, Laser Velocimetry and Particle Sizing, eds. H. D. Thompson and W. H. Stevenson, Hemisphere, p. 267.

Dyrkacz, G. R., C. A. A. Bloomquist, L. H. Fuchs and E. P Horwitz, 1981 , in Chemistry and Physics of Coal Uitlization - 1980 (APS, Morgantown) (edited by B. R. Cooper and L. Petrakis, New York, 1981) AIP Conference Proceedings, No. 70.

Eckbreth, A. C., P. A. Bonczyk, and J. F. Verdieck, 1978, Appl. Spec. Revs.13, 15.

Elofson, R. M. and K. F. Schulz, 1967, Am. Chem. Soc. Div. Fuel Chem. Prepr.11, 513.

Finkelman, R. B., 1978, in Scanning Electron Microscopy, Vol. 1, SEM Inc., AMF O'Hare (Chicago), IL 60666, USA, p. 143.

Friedel, R. A. and J. A. Queiser, 1959, Fuel
$38,369$.

Friedel, R. A., J. A. Queiser, and G. L. Carlson, 1971, Am. Chem. Soc. Div. Fuel Chem. Prepr. 15, 123.

Froitzheim, H., $\overline{1977, ~ " E l e c t r o n ~ E n e r g y ~ L o s s ~}$ Spectroscopy" in Electron Spectroscopy for Surface Analysis, ed. H. Ibach, Springer-Verlag, Berlin.

Frost, D. C., W. R. Leeder and R. L. Topping, 1974, Fuel 53, 206-11.

Fujii, S., 1963, Fuel 42, 17; 341.

Gibbon, D. L., 1979, in Scanning Electron Microscopy, Vol. 1, SEM Inc., AMF O'Hare (Chicago) IL 60666, USA, p. 501.

Gluskoter, H. J., R. R. Ruch, W. G. Miller, R. A. Cahill, G. B. Dreher and J. K. Kuhn, 1977, "Trace Elements in Coal: Occurance and Distribution," Illinois State Geological Survey, Circular 499, p. 109.

Goldstein, J. I., and N. Yakowitz, 1975, Practical Scanning Electron Microscopy, Electron and Ion Microprobe Analysis, Plenum Press, New York.

Goodman, D. W., R. D. Kelly, and T. E. Madey, 1980, J. Catal. 63, 226.

Goulard, R. and P. J. Emmerman, 1980, Topics in Current Physics 20.

Graham, S. C., A. J. Grant, and. J. M. Jones, 1974, AATA Journal, 121140.

Grandy, D. W. and L. Petrakis, J. Mag. Res. (in press).

Greer, R. T., 1979, in Scanning Electron Microscopy, Vol. 1, SEM Inc., AMF O'Hare (Chicago), IL 60666, USA, p. 477.

Guidoboni, R. J., 1978, in Analytical Methods for Coal and Coal Products, ed. Clarence Karr, Jr; Vol. 1 (1978), pp. $421-434$.

Hamilton, J. C., N. Swanson, B. J. Waclawski and R. J. Celotta, 1981, J. Chem. Phys. (to be published, private communication). Hanson, R. L., and N. E. Vanderborgh, 1980, in Analytical Methods for Coal and Coal Products, ed. Clarence Karr, Jr.; Vol. 3, p. 73.

Hardesty, D. R., 1980, (private communication).

Harding, L. B., 1981, J. Phys, Chem. 85, 10.

Harris, L. A., and C. S. Yust, 1979, Carbon $17,307$.

Harris, L. A., and C. S. Yust, 1976, Fuel $55,233$.

Harris, L. A., D. Braski and C. S. Yust, 1977, Microstruct. Sci. 5, 35.

Harvey, A. B., and J. W. Nibler, 1978, Appl. Spec. Rev. 14, 101. 
Hirsch, P. B., 1954, Proc. Roy. Soc., (London) A 226, 143.

Hodgson, K. O., and S. Doniach, 1978, Chem, and Eng. News, August 21, 1978, p. 26.

Hombach, H. B., 1980, "Fuel," Vol. 59, No. 7, 465 and refs. therein.

Hombach, H. B., Erdol, Kohle, and Erdgas, 1979, Petrochem. Brennst. Chem., Vol. $32,134$.

Howard, C. J., 1979, J. Phys. Chem. 83, 3.

Huggins, F. E., and G. P. Huffman, 1978 , 1979, in Analytical Methods for Coal and Coal Products, ed. Clarence Karr, Jr.; Vols.1, 2., (1978), vol. 3 (1979), Academic Press, Chapter 50.

Huggins, F. E.,, D. A. Kosmack, G. P. Huffman, and R. J. Lee, 1980, Scanning Electron Microscopy, SEM Inc., AMF O'Hare (Chicago), IL 60666,. USA, Vol. 1 p. 531.

Humphreys-Owens, S. P. F., and L. A. Gilbert, 1958, Proc. Conf. Ind. Carbon and Graphite, London, p. 37.

Hurst, G. S., M. G. Payne, S. D. Kramer, and J. P. Young, 1979, Rev. Mod. Phys. 51, 767.

Ibach, H., 1977, Electron Energy Loss Spectroscopy in Electron Spectroscopy for Surface Analysis, ed. H. Ibach, Springer-Verlag, Berlin.

Karr, Clarence, Jr.; 1978, 1979, Analytical Methods for Coal and Coal Products, Vols. 1,2, vol.3), Academic Press.

Klick, D., K. A. Marko, and L. Rima, 1981, Appl. Optics 20, 1178.

Kosky, P. G., E. J. Lamby, D. H. Maylotte, R. L. St. Peters, and C. Spiro, 1981, Am. Chem. Soc., Atlanta, March 1981.

Koumelis, C. N., and C. A. Londos, 1980, Can. J. Phys. 58, 1507.

Lane, N. F., 1980, Rev. Mod. Phys. 52, 29.

Lapp, M., L. M. Goldman, and C. M. Penney, 1972, Science 171112.

Larsen, J. W., and J. Kovac, 1978, in Organic Chemistry of Coal, ed. J.W. Larsen, ACS Symposium Series 71, 36.

Lee, P. A., 1976, Phys. Rev. B 13, 5261.

Levinson, L. M., 1981, in Chemistry and Physics of Coal Utilization-1980 (APS, Morgantown) (edited by B. R. Cooper and L. Petrakis, New York, 1981), AIP Conference Proceedings No. 70.

Lifshitz, A., ed. Shock Waves in Chemistry and Chemical Technology (Decker, NY, in press).

Lin, J. S., R. W. Hendricks, L. A. Harris, and C. S. Yust, 1978, J. Appl. Crystal. 11 , 621 .
Low, I. J., 1959, Phys. Rev. Lett. 2, 285.

Lucht, L. M. and N. A. Peppas, 1981, Chemistry and Physics of Coal Utilization -1980, edited by B. R. Cooper and L. Petrakis, New York, AIP Conference Proceedings No. 70.

Maciel, G. E., M. J. Sullivan, N. M. Szeverenyi, and F. P. Miknis, 1981, Chemistry and Physics of Coal Utilization -1980, edited by B. R. Cooper and L. Petrakis, AIP Conference Proceedings No. 70.

Maekawa, Y., T. Yoshida, Y. Yoshida, M. Imanari, 1977, J. of Jap. Petrol. Inst. Vol. 56351 .

Makovsky, L. E., P. Waldstein, and W. H. Edwards, 1971, Nature (Phys. Sci.) 231, 154.

Marino, R. A., H. R. Wenk, J. A. Apps and S. M. Kaliner, 1980, J. Molec. Structure 58, 445.

Maxwell, B. E., H. R. Child and A. H. Nartern, ORNL Solid State Division Progess Report (in press).

Maylotte, D. H., 1981, Bull. Am. Phys. Soc. $26,201$.

Maylotte, D. H., J. Wong, F. W. Lytle, R. B. Greegor and R. L. St. Peters, 1981, submitted to Science.

Mentser, M., H. J. O'Donnell and S. Ergun, 1962, Fuel 41, 153.

Michael, J. V., and J. H. Lee, 1979, J. Phys. Chem. 83, 10.

Miyagawa, I., and C. Alexander, 1981, in Chemistry and Physics of Coal Utilization - 1980 (APS, Morgantown) edited by B. R. Cooper and L. Petrakis, New York, 1981), AIP Conference Proceedings No. 70.

Miyagawa, I., and C. Alexander, 1979, Nature (London) 278, 40.

Moza, A. K., L. G. Austin and G. G. Johnson, Jr., 1979, in Scanning Electron Microscopy, Vol. 1, SEM Inc. AMF O'Hare (Chicago), IL 60666, USA, p. 473.

Neavel, R. C., 1979, American Chemical Society, Division of Fuel Chemistry, Preprints 24, 73.

Neavel, R. C., 1980, Am. Chem., Soc., Div. of Fuel Chem. Preprints 25, 246.

Ondov, J. R., W. H. Zoller, I. Olmez, N. K. Aras, G. E. Gordon, L. A. Rancitelli, K. H. Abel, R. H. Filbey, K. R. Shah and R.C. Ragaini, 1979, Anal. Chem., 47, 1102.

Orchin, M., C. Golumbic, J. E. Anderson, and H. H. Storch, 1951, U.S. Bur. Mines, Bull. No. 505. 
Pan, L. S., 1967, Pressure Effects on Laboratory Coalification Processes, Ph.D. dissertation, University of Utah.

Patterson, J. H., A. L. Turkevich and E. Franzgrote, 1966, Radiosotope Instruments in Industry and Geophysics, Vol. 1, p. 337 (IAEA).

Pavlovic, A. S., J. M. Cook, J. J. Renton, 1981 , in Chemistry and Physics of Coal Utilization -1980 (APS, Morgantown) (edited by B. R. Cooper and L. Petrakis, New York, 1981), AIP Conference Proceedings No. 70.

Petrakis, L., and D. W. Grandy, 1978, Anal. Chem. 50303.

Petrakis, L., and D. W. Grandy, 1981, in Chemistry and Physics of Coal Utilization -1980 (APS Morgantown) (edited by B. R. Cooper and L. Petrakis, New York, 1981a), AIP Conference Proceedings No. 70.

Petrakis, L. , and D. W. Grandy, 1981b, Nature 289, 476.

Pierce, T. B., 1974, in Characterisation of Solid Surfaces, eds. P. F. Kane and G. B. Larrabee, Plenum, N.Y., 1974.

Pines, A., M. G. Gibby, and J. S. Waugh, 1973, J. Chem. Phys. 59, 569.

Prather, John W., J. A. Guin and A. R. Tarrer, 1978, in Analytical Methods for Coal and Coal Products, ed. Clarence Karr, Jr., Vol. 1, p. 357, Academic Press (1978).

Prather, John W., James A. Guin and Arthur R. Tarrer, 1979, op. cit. Vol. 3, Ch. 49.

Quate, C. F., 1981, in Chemistry and Physics of Coal Utilization -1980 (APS, Morgantown) (edited by B. R. Cooper and L. Petrakis, New York, 1981), AIP Conference Proceedings No. 70.

Raymond, R., and R. Gooley, 1978, in Scanning Electron Microscopy, Vol. 1, SEM Inc., AMF O'Hare (Chicago), IL 50666, USA, 1978, p. 93.

Raymond, R., Jr., and R. Gooley, 1979, in Analytical Methods for Coal and Coal Products, ed. Clarence Karr, Jr., Vol. 3, Ch. 48, Academic Press.

Retcofsky, H. L., 1977, Appl. Spectroscopy $31,116$.

Retcofsky, H., 1980, private communication.

Retcofsky, H., M. R. Hough, and R. B. Clarkson, 1979 , American Chemical Society, Division of Fuel Chemistry, Preprints 24, No. 1, 83 (1979).

Rockley, M. G., 1980a, Appl. Spect. 34, 405. Rockley, M. G., 1979, Chem. Phys. Lett. 68, 455.
Rockley, M. G., I980b, Proc. IEEE Ultrasonics Symposium, Boston, Nov. 5-7, 1980.

Rockley, M. G., and J. P. Devlin, 1980 , Appl. Spectroscopy 34, 407.

Roy, M. M., 1957, Fuel 36, 249.

Royce, B. S. H., Y. C. Teng, and J. Enns, 1980, Proc. IEEE Ultrasonics Symposium, Boston, Nov. 5-7, 1980.

St. John, G. A., S. E. Buttrill, Jr., M. Anbar, 1978, ACS Symposium Series 71, 223.

Scanning Electron Microscopy, SEM Inc., AMF O'Hare (Chicago). Published yearly.

Schlick, S., P. A. Narayana and L. Kevan, 1978, J. Am. Chem. Soc. 100, 3322.

Schmidt, P. W., M. Kalliat and C. Y. Kwak, 1981 , in Chemistry and Physics of Coal Utilization -1980 (APS, Morgantown) (edited by B. R. Cooper and L. Petrakis, New York 1981), AIP Conference Proceedings No. 70 .

Schuyer, J., and D. W. van Krevelen, 1959, Fuel 33, 176.

Sharkey, A. G., Jr., J. L. Shultz, and R. A. Friedel. 1964, Nature 202, 988.

Shinn, J. H., and T. Vermuelen, 1979, in Scanning Electron Microscopy, Vol 1, SEM Inc., AMF O'Hare (Chicago), IL 60666, USA, p. 487.

Sibener, S. J., R. J. Buss, P. Casavecchia, T. Hirooka, and Y. T. Lee, 1980, J. Chem. Phys. 72, 4341.

Siegbahn, S. et al.,1967, ESCA, Almquist and Wiksells Boktryckeri, $\overline{A B}$, Uppsala, Sweden.

Silbernagle, B. G., L. B. Ebert, R. H. Scholosberg, and R. B. Long, 1980, ACS Advances in Chemistry series, Vol. 92, Chapter 3 .

Sinfelt, J. H., and G. H. Via, 1978, J. Chem. Phys. 68, 2009.

Smith, J. A. S., 1980, J. Molec. Structure $58,1$.

Solomon, P. R., 1981, in Chemistry and Physics of Coal Utilization - 1980 (APS, Morgantown) (edited by B. R. Cooper and L. Petrakis, New York, 1981), AIP Conference Proceedings No. 70.

Somorjai, G. A., 1979, Surface Science 89, 496.

Somor jai, G. A., and M. A. van Hove, 1979, Adsorbed Monolayers on Solid Surfaces in Structure and Bonding Vol. 38 , Springer-Verlag, Berlin.

Speight, J. G., 1971, Appl. Spect. Rev. $\underline{5}$, 211. 
Stanton, S. W., and R. B. Finkelman, 1979, in Scanning Electron Microscopy, Vol. 1, SEM Inc. AMF O'Hare (Chicago), IL 60666, USA, p. 456.

Steinnes, E., 1979, in Analytical Methods for Coal and Coal Products, ed. Clarence Karr, Jr., Academic Press, Vol. 3, Ch. 46.

Stohr, J., D. Denleyk, and P. Perfetti, 1978, Phys. Rev. B 18, 4132.

Strehlow, R. A., L. A. Harris and C. S. Yust, 1978, Fuel 57, 185.

Sumino, K., R. Yamamoto, F. Hatayama, S. Kitamura, H. Itoh, 1980, Anal. Chem. 52, 1064.

Teichmuller, M. and R. Teichmuller, 1966, Coal Science, 55, Advances in Chemistry Series, R. F. Gould, ed., Washington, D.C., American Chem. Soc., 133-155.

Thiel, P. A., W. H. Weinberg and J. T. Yates, Jr., 1979, J. Chem. Phys. 71, 143.

Thompson, R. R., I981, in Chemistry and Physics of Coal Utilization -1980 (APS, Morgantown) (edited by B. R. Cooper and L. Petrakis, New York, 1981), AIP Conference Proceedings No. 70.

Ting, F. T. C., 1978, in Analytical Methods for Coal and Coal Products, ed. Clarence Karr, Jr., Academic Press, Vol. 1, Ch. 1.
Tsukashima, H., 1967, Fuel, 46, 177.

van Krevelen, D. W., H. A. G. Chermin and J. Schuyer, 1959, Fuel 38483.

van Krevelen, D. W., and J. Schuyer, 1957, in Coal Science, Elsevier.

Van oostrom, A., Surface Sci., 89615 (1979).

van Vucht, H. A., B. J. Rietvald, and D. W. van Krevelen, 1955, Fuel 34, 50.

Vidal, C. R., 1980, Opt. Lett. 5, 158.

Volborth, A., 1979a, in Analytical Methods for Coal and Coal Products, ed. Clarence Karr, Jr., Academic Press, Vol. 3., Ch. 47.

Volborth, A., 1979b, op. cit., Vol. 3, Ch. 55.

West, P. R., and S. E. Cannon, 1981, in Chemistry and Physics of Coal Utilization - 1980 (APS, Morgantown) edited by B. R. Cooper and L. Petrakis, New York), AIP Conference Proceedings No. 70.

Wittmaack, K., 1979, Surface Science 89, 668.

Wong. J., D. H. Maylotte, F. W. Lytle, R. G. Greegor and R. L. St. Peters, 1981, Bull. Am. Phys. Soc. 26, 320 (1981).

Wyatt, P. J., 1980, Appl. Opt. 19, 975.

Ziegler, L. D., and B. Hudson, $1981, \mathrm{~J}$. Chem. Phys. 74, 982. 


\section{RESEARCH FOR THE TECHNOLOGIES IV. A. PRIMER ON COAL UTILIZATION TECHNOLOGIES}

Empirically based coal technology has a long history (Lowry, 1963; Elliott, 1981); its industrial development was well advanced by mid-19th century. In recent times the drive for ever higher efficiency in coal fired boilers and the need for petroleum substitutes on a large scale have inspired efforts to develop science-based technologies covering a wider range of applications and concerns than the traditional ones. The task is very difficult -- partly because of the inherently variable and ill defined nature of coal itself, partly because multiphase flows and chemical reactions at solid surfaces are intrinsic features of the technology, and partly because the hetero-atom and mineral content of coal gives rise -- whatever the mode of use -- to toxic or harmful residues which must be controlled.

Efforts in the United States to develop and refine modern coal technology are largely financed by industry and by the U.S. Department of Energy. The effort is broad in scope and of substantial size. (Fumich, 1980)

\section{Plan of presentation}

This section contains brief introductory characterizations of the most important technologies currently in use or under development; topics discussed include:

$$
\text { pyrolysis }
$$

flow conditions in combustion and conversion processes

gasification

direct liquefaction

indirect liquefaction

direct combustion

magneto-hydrodynamic conversion (MHD)

combined cycles and co-generation

high temperature fuel cells mining and coal preparation

Pyrolysis, the general phenomenology of gasification, and flow conditions are discussed first since they are of general and recurrent interest. Discussion of these is followed by descriptions of several specific processes.

\section{Pyrolysis}

Heating of the coal to the point of structural transformation and partial decomposition is an initial step in several important technologies, specifically including direct combustion, MHD, and gasification. The transformation and partial decomposition of coal upon heating is termed "pyrolysis" or, in various contexts and with differing emphases, "devolatilization" or "distillation".

When coal, below the rank of anthracite, is heated (usually in the absence of air) large amounts of gases and volatile tars and oils are driven off. The tars and oils are highly aromatic in character and have a stoichiometry of approximately $\mathrm{CH}$. The gases include methane, hydrogen, carbon monoxide, carbon dioxide, hydrogen sulfide and ammonia. Overall, most of the hydrogen and, typically, about $35 \%$ of the carbon in the original coal is incorporated in these volatiles. The residue is termed "char" and is a mixture of carbon, mineral matter, and some residual hydrocarbons.

The character of the char and the amount of volatiles driven off depend strongly both on the original coal and on the circumstantial details of the heating. (With very rapid heating rates achievable in the laboratory, for example, some coals may give off upward of $60 \%$ of their carbon in the volatiles.)

Changes taking place in the condensed phase during pyrolysis depend markedly upon the coal used; they may include swelling and softening and, in consequence, some degree of agglomeration or "caking" and the development of macroscopic porosity such as is familiar in coke. In fact coke is one specialized kind of char, and the principal direct industrial application of pyrolysis is coke making. Coke making for the steel industry is so arranged that the volatiles recovered are economically important, representing about $35 \%$ of the process output value.

The coking behavior of a specific coal can be fairly well predicted from rank and petrography; for example coals of very high and very low rank exhibit little softening and swelling and are not suitable for coke making. Nevertheless, scientific understanding of the detailed mechanisms involved in pyrolysis is very meagre. (Anthony and Howard, 1976; Solomon and Colket, 1977)

\section{Synthetic fuels}

Direct combustion of coal, though highly developed and in its modern forms quite efficient, is not very suitable for transport 
power or for other small scale operation. Considerable importance, therefore, attaches to conversion of coal into clean gaseous and liquid fuels which can serve as substitutes for petroleum products. That this is possible was shown long ago. Germany, in WWII, manufactured commercial quantities (small relative to present U.S. consumption) of synthetic liquid fuel from coal, as does South Africa today.

Historically, new factors now give rise to a need for major improvements of the earlier technology. These factors include the scale on which it is desired to produce synthetic fuels and -- partly because of scale - the intense concern for environmental effects. (U.S. consumption of energy is now about 3.5 times what it was four decades ago.) Economically, moreover, there is a great difference between the "cost-no-object" context of a war or embargo situation and the routine supply of motor fuel for general public use. Therefore, considerable effort is now going into the cleaning up and scaling up of gasification and liquefaction technology and making it more efficient.

In contemplating the development of these synthetic fuel technologies one must recognize that cleaning the coal is a major objective, no less important than changing its physical state. SRC-I, for example, is commonly discussed as a "liquefaction" process, but it yields a product (having the appearance of pitch) which is not a liquid at ordinary temperatures. The SRC-I output, however, is much lower in mineral matter and other impurities and somewhat higher in hydrogen content than the starting coal. By the same token, many gasifiers yield a product that is suitable neither for storage, nor for shipment, and is intended rather for immediate on-site use in some further process.

\section{Gasification, importance and general features}

Gasification plays an essential role in most processes for transformation or indirect use of coal, so it is logical to begin the discussion with gasification which, in any case, is at the heart of fuel gas and substitute natural gas production processes. For indirect liquefaction processes, gasification is the key step which produces synthesis gas ( $\mathrm{CO}$ and $\mathrm{H}_{2}$ ), while for the direct liquefaction processes, some kind of gasifier is usually relied upon to produce the requisite large quantities of hydrogen. Thus, coal gasification may be addressed to any one of three different objectives - - the production of $\mathrm{CO}$ and $\mathrm{H}_{2}$ mixtures ("synthesis gas"), the production ${ }^{2}$ of $\mathrm{CH}_{4}$ (substitute natural gas or "SNG"), or the production of hydrogen.

Economically, the gasifier and its associated auxiliary units are often the most expensive part of a coal conversion plant. Gasifier operation is poorly understood, and it seems likely that the potential exists for large reductions in cost and increases in efficiency. The various flow, instrumentation, and materials problems offer an excellent opportunity for physicists to contribute importantly to a most critical part of coal conversion technology.

A recent report (National Academy of Sciences/National Research Council, 1977) 1 ists and very briefly describes 37 representive processes for the production of low- and intermediate-BTU fuel gases, grouped into 8 major categories. All are designed to implement much the same basic strategy:

1. Pyrolyze the coal.

2. React the char with steam and oxygen (or air) -- at high temperature ( 800 to 900 C) and, in some cases, at elevated pressure:

$$
\mathrm{C}+\mathrm{H}_{2} \mathrm{O}+31 \cdot 38 \mathrm{kcal} \stackrel{\leftarrow}{\rightarrow} \mathrm{CO}+\mathrm{H}_{2}
$$

In addition to being highly endothermic (as noted), this reaction exhibits a substantial activation barrier.

The heat necessary for the pyrolysis and for the carbon-steam reaction is supplied within the gasifier, in most cases, by burning a fraction of the input coal in situ; and it is this which necessitates the feeding either of air or of oxygen.

The kinetics of the (solid) carbon reactions with the mixture of steam and other molecular species present in the gasifier are poorly understood even in the case of pure carbons. In the much more complicated case of coal, naturally occuring mineral constituents play a significant role in catalysis of the heterogeneous reactions. These minerals are diverse and highly dispersed within the coal (Gluskoter, 1975; Jensen, 1975). The chemical attack is complex involving a multi-step process such as adsorption, reaction, and desorption at preferred carbon sites (McKee, 1974, 1979). 
A general concommitant of the carbon steam reaction is the water gas shift reaction:

$$
\mathrm{CO}+\mathrm{H}_{2} \mathrm{O} \stackrel{\leftarrow}{\leftrightarrows} \mathrm{CO}_{2}+\mathrm{H}_{2}+9.83 \mathrm{kcal}
$$

Kinetically, the shift reaction usually is in equilibrium in the gasifier. But, depending upon objectives, the product mix may later be deliberately shifted in a separate catalytic step which employs the same shift reaction but under conditions appropriate to a different equilibrium.

Further reactions which can and - - to varying degrees depending upon operating conditions -- do take place in gasifiers are:

$$
\begin{aligned}
& \mathrm{C}+\mathrm{CO}_{2} \stackrel{\leftarrow}{\leftrightarrows} 2 \mathrm{CO} \\
& \mathrm{C}+2 \mathrm{H}_{2}^{2} \stackrel{\leftarrow}{\leftrightarrows} \mathrm{CH}_{4}
\end{aligned}
$$

The essential gasifier output in any case is a mixture (primarily) of hydrogen and carbon monoxide. Operating conditions determine the amount of residual methane, carbon dioxide, and water. Methane, in most instances, is a minority constituent resulting almost entirely from the pyrolysis step of the process.

Different gasifier designs operating with different feed coals produce a wide range of product gas qualities. In most cases these are classified as "low BTU" ( 100 to 200 BTU per SCF, i.e. "standard cubic foot") and "intermediate BTU" (275 to 500 BTU per SCF). These heating values may be compared with natural gas which is primarily methane and which is considered "high BTU" at 900 to 1100 BTU per SCF. Usually the gasifiers fed with air produce gas which is diluted with atmospheric nitrogen and therefore is low BTU, while those fed with oxygen produce intermediate BTU mixtures (little or no $\mathrm{N}_{2}$ ).

It is generally considered economical to transport intermediate BTU gas over distances up to 100 miles for use as fuel in industrial furnaces, boilers, power stations, etc. Most applications contemplated for low BTU gas, on the other hand, involve "over-the-fence" utilization in some kind of integrated or

\footnotetext{
Note, however, that if the main purpose is to produce methane, it is possible to adjust the $\mathrm{H}_{2} / \mathrm{CO}$ ratio by means of the water gas shift ${ }^{2}$ reaction and to follow this by the catalytically mediated reaction:

$\mathrm{CO}+3 \mathrm{H}_{2} \rightarrow \mathrm{CH}_{4}+\mathrm{H}_{2} \mathrm{O}+49.27 \mathrm{kcal}$

accomplishing the desired result.
}

hybrid process, e.g. in fuel cells, gas turbines, etc. In such cases obtaining a chemically reactive gas or a clean gas is the primary objective of gasification.

The regime in a gasifier is very complex. Elements other than carbon are present in the char; numerous reactions take place (some even proceeding in opposite directions in different parts of the reactor); and many substances are present in the product in addition to hydrogen, carbon monoxide, methane, (and, of course, unreacted water and carbon dioxide).

Ash

Every kind of coal utilization technology must incorporate some practical means of coping with the ash which originates from inorganic constitutents of the feed coal. It has not proven possible within the limitations of this report to adequately discuss -- or even to enumerate -- the many chemical, physical, and morphological variations of coal ash and the associated range of technical problems. These problems are, nevertheless, of very great importance. For example, one of the defining characteristics of a gasifier configuration is the method of controlling ash and removing it from the reaction chamber. Reactor temperature may be kept below ash fusion point and the ash removed dry; ash may be allowed to melt and be removed as "slag"; or it may be allowed to agglomerate to some controlled degree, and even used as a vehicle for heat transport.

\section{Flow regimes in heterogenous reactors}

In characterizing gasifiers and other coal utilization processes one must consider not only the chemistry but also, and equally important, the physical arrangements by which the coal and other reactants are heated and kept in contact and the reaction products removed. Even though the chemistry, in broad outline, may remain the same, physical arrangements can strongly affect the efficiency and reliability of the process, the composition of the product, and -- most important, perhaps -- the kinds of coal that can be used as feed.

Because coal is solid, its use either in direct combustion or for conversion to synthetic fuels requires the contacting of 
solid coal with flowing gases and liquids. Thus some discussion of gas-solid, liquid-solid, and gas-liquid-solid flow is an important part of any description of coal technology. Multiphase flows, moreover, offer many interesting and challenging unsolved problems in classical physics. At this point, therefore, we present a quick overview of the various gross phenomena that are exhibited in multiphase flow and the terminology prevalent in their description. Examples of these phenomena in coal utilization and conversion recur throughout the remainder of the report. (See, especially, Section IV C.)

The flow systems in chemical and combustion reactors used in industry can be divided into three principal classes: systems in which the solid phase is fixed; systems in which the solid phase moves slowly; and systems in which the solid phase is in a mobile or suspended state due to the motion of the gas or liquid phases (fluidized beds).

\section{Fixed beds}

The simplest are fixed-bed gas-solid systems (Smith, 1970; Carberry, 1976) in which gas passes continuously over stationary solid particles or granules. Such fixed-bed systems are most frequently encountered in coal conversion technology as beds of solid granular catalyst through which coal-derived gases flow for conversion to more useful products. The heat and mass transport phenomena for these are better understood than those for any other gas-solid flow systems.

More complex are three phase systems that have simultaneous flows of both gases and liquids through a fixed bed of granular solids. Such systems may be arranged to have the gas and liquid flow cocurrently downwards, cocurrently upwards, or countercurrently to each other. The hydrodynamics and the heat and mass transfer considerations are different for each case.

A widely used configuration for three-phase systems is trickle flow in which the liquid flows downward through a bed of granular solids in the form of a thin liquid film and the gas flows in a continuous phase between the solid particles either cocurrently or countercurrently. In the most common mode of operation, gas and liquids flow cocurrently downward.

When liquid and gas flow cocurrently upwards there are, of course, no thin films, and fixed bed operation under these circumstances normally displays one of three regimes: bubble flow (at low liquid and gas rates), pulsating flow (at high gas rates), and spray flow. In bubble flow, the gas is the dispersed, phase and the liquid is continuous. In pulsating flow, pulses of gas and liquids pass through the reactor. In spray flow, the gas is a continuous phase, and the liquid is dispersed.

\section{Moving beds}

In moving-bed flows the granular solids are allowed to move (slowly) downward under gravity. The flow of solids in the vessel proceeds in a rod-like fashion with the relative positions of the granules in the bed remaining essentially unaltered. Gas flow can be either upward or downward, but in the case of upward flow the velocity of the gas must not be so great as to expand the solid bed into a fluidized state. The bulk density of a moving bed has been found to correspond closely to the loosest packing arrangement for fixed beds.

Moving-bed reactors can be arranged for three-phase flows similar to those employed with fixed beds. When the liquid and gases flow cocurrently upwards, pulsating and spray conditions can be realized. In some moving bed coal reactors pulsating conditions are used to keep the solid coal from forming plugs in the reactor. The moving bed is much used in coal gasification reactors and in coal handling systems.

\section{Suspended particles and fluidized flows}

Fluidized flow (Davidson and Harrison, 1971; Kunii and Levenspiel, 1969) is a very interesting and useful flow condition known to offer many advantages for chemical reactor design. It has not yet been used as much as it probably would be with more detailed understanding; lack of scientific understanding of the phenomena makes scale-up of reactors uncertain and expensive. Despite this difficulty, fluidized flow has been used both in conversion reactors and in combustion, as well as for the transportation of solids. This area would appear to offer physicists a particularly rich field for making substantial contributions to coal technology. 
Figure IV-A-1 (Davidson and Harrison, 1971) indicates the basic flow configurations possible for systems of suspended particles. Figure IV-A-2 (Davidson and Harrison, 1971) suggests some of the regimes that can exist in a fluidized bed and defines some of the terms associated with the phenomena.

As shown in Figure IV-A-1, there are three basic vertical-flow configurations: (1) fluid and solids flowing cocurrently upward, (2) fluid and solids flowing cocurrently downward, and (3) fluid flow upward with countercurrent solid flow downward. Each of these three vertical-flow conditions can occur under varying degrees of solids concentration, represented schematically in Figure IV-A-1.

Homogeneous gas-solids mixtures generally belong to concentration ranges referred to as dense or disperse (dilute). Intermediate densities generally exhibit non-homogeneous flow regimes involving alternate slugs of gas and solids, or bubbles of gas within a fluidized mass. Liquid-solid systems, on the other hand, do not usually exhibit bubble formation or slugging; and, thus approach more nearly an ideal system in which homogeneous concentrations from zero to the bulk density can be maintained.

In general, particulate fluidization without bubbling occurs with liquid-solid

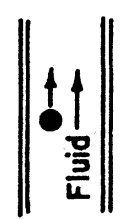

(1) Cocurren Upflow

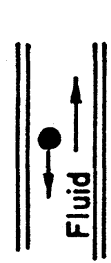

(3) Countercurrent

Flow

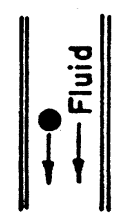

(2) Cocurrent Downflow

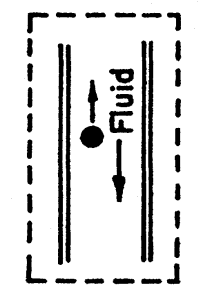

Countercurrent

Flow; $\left\{>P_{S}\right.$

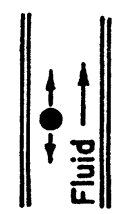

Zero Net Solids Flow (fluidized)

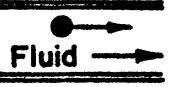

Horizontal Conveying
Directions of motion in fluid-particle systems.

FIG. IVA-1. Flow conditions in systems of suspended particles (Davidson and Harrison, 1971). systems, or with gas-solid systems when the particles are very fine, and in the latter case over only a limited range of velocities. Practical application of this regime is rare. Aggregative fluidization, however, occurs with all other gas-solid systems and sometimes -when the solids are of high density -- with liquid-solid systems. Aggregative fluidization is characterized by gas bubbles rising through a bed of minimally fluidized particles. How and why bubbles form in such fluidized-solid systems is little understood. If bubble sizes approach the diameter of the bed, slugging will occur and one refers to a "slugging bed." As the gas velocity is further increased, the bubbling or slugging regime breaks down into a state called "turbulent fluidization." "Fast fluidization" is an extension of the turbulent fluidization regime. The principal distinction between the two is that in fast fluidization, fluidized density is strongly dependent only upon solids feeding rate.

Entrained flow or dilute phase pneumatic conveying is characterized by solid particles completely suspended in the gas-phase. The flow patterns in either horizontal or vertical entrained flow are rather complex and are affected by the solid-to-gas ratios.

Major advantages of fluidized bed reactors are that they give flexibility of mixing, of heat recovery, and of temperature control. They allow the use of fine catalyst particles, which minimize the intraparticle diffusion effects. On the other hand, they

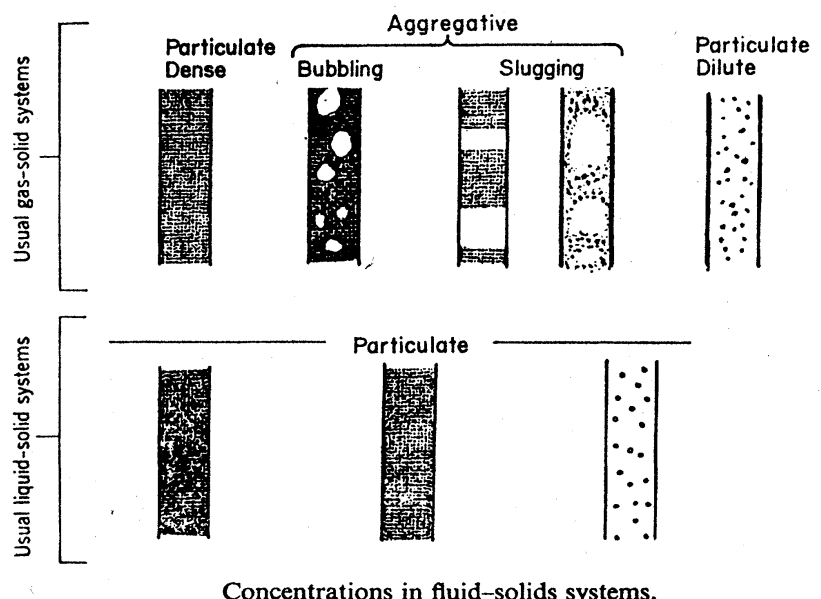

FIG. IVA-2. Regimes in a fluidized bed (Davidson and Harrison, 1971). 
may, if not designed properly, give poor conversion due to axial mixing. And separation of catalyst from product mixture may be difficult.

Table IV-A-1 summarizes the characteristics of various gas-solid systems and identifies practical examples of application to coal technology. Table IV-A-2 summarizes the same kind of information for gas-liquid-solid systems.

\section{Some specific gasifiers}

Typical gasifier reactor configurations are depicted in Figures IV-A-3, IV-A-4, and IV-A-5. These are, respectively: entrained gasifier, moving bed gasifier, and fluidized bed gasifer.

The entrained gasifier sprays a slurry of fine coal particles suspended in oil or water into a chamber and ignites it in an oxygen (or air) atmosphere. This system behaves rather like a combustor; the coal is immediately pyrolyzed, and the residual char reacts with the steam or burns until the oxygen is consumed. One advantage of an entrained gasifer is that it operates at such high temperature (1350-1550 C) that all the tars arising from the initial devolatilization are converted to simple gases such as $\mathrm{CO}, \mathrm{CO}_{2}$, $\mathrm{H}_{2} \mathrm{O}, \mathrm{H}_{2}$, $\mathrm{H}_{2} \mathrm{~S}$ and trace impurities. ${ }^{2} \mathrm{~A}$ concommitant ${ }^{2}$ isadvantage is an exit gas temperature so high that the ash is in a molten state ("slagging" operation); and, in spite of an inertial separation device (a pool at the base of the reactor), the exit gas must be cooled (by mixing with recycled cooled gas) to solidify molten droplets of slag that remain. The overall efficiency of the system, moreover, is critically dependent on heat recovery from the exit gas. (In practice a

TABLE IV-A-1. Flow regimes and phenomena in gas-solid systems.

\begin{tabular}{|c|c|c|}
\hline Regime or Phenomenon & Characteristics & Practical Examples \\
\hline Fixed bed & Stationary particles w/gas flow & Coal gasification: Lurgi \\
\hline $\begin{array}{l}\text { Particulate (homogeneous } \\
\text { fluidization }\end{array}$ & $\begin{array}{l}\text { Particles uniformly expanded, no } \\
\text { bubbles (small particles, magneti- } \\
\text { cally stabilized bed) }\end{array}$ & \\
\hline $\begin{array}{l}\text { Aggregative or bubbling } \\
\text { (heterogeneous) fluidization }\end{array}$ & $\begin{array}{l}\text { "Open" gas bubbles rising through } \\
\text { minimally fluidized particles }\end{array}$ & $\begin{array}{l}\text { Coal gasification } \\
\text { Coal pyrolysis: COED }\end{array}$ \\
\hline Turbulent fluidization & $\begin{array}{l}\text { Large eddy motions, absence of } \\
\text { permanent bubbles or slugs }\end{array}$ & \\
\hline Fast fluidization & $\begin{array}{l}\text { Low density solids phase; backmixing } \\
\text { of particle aggregates }\end{array}$ & \\
\hline Entrained flow & Dilute phase transport of particles & $\begin{array}{l}\text { Coal gasification } \\
\text { Coal combustion } \\
\text { Coal pyrolysis }\end{array}$ \\
\hline \multicolumn{3}{|l|}{ Special Cases } \\
\hline Moving bed & $\begin{array}{l}\text { Particles move downward in fixed-bed } \\
\text { condition }\end{array}$ & Coal gasification \\
\hline Spouted bed & $\begin{array}{l}\text { Vertical gas jet in shallow packed } \\
\text { bed forms stable spout, entrains } \\
\text { and recycles solids }\end{array}$ & Coal combustion \\
\hline Slugging bed & $\begin{array}{l}\text { Bubbles agglomerate to slugs fill- } \\
\text { ing cross-section of bed }\end{array}$ & \\
\hline
\end{tabular}


TABLE IV-A-2. Flow regimes and phenomena in gas-liquid-solid systems.

Regime or Phenomenon

Gas-liquid-fixed-bed-solid

1. Trickle bed

2. Cocurrent-upflow
Characteristics

Solids in fixed-bed, liquid flows downward in thin liquid film, gas flows in continuous phase

Bubble-flow (gas in dispersed phase, liquid in continuous phase) or pulsating-flow (both gas and liquid in pulses) or spray-flow (gas in continuous phase, liquid in dispersed phase)
Practical examples
Various hydroprocessing processes including upgrading of coal-derived liquids.

Coal liquefaction:

SYNTHOIL process

Fischer-Tropsch process

(Other cases: Cocurrent-downflow, countercurrent-flow, and segmented fixed-bed reactors are less used in coal technology)

Gas-liquid-suspended-solid

1. Continuous slurry or fluidized-bed

Coal liquefaction:

H-coal, SRC, EDS processes

Fischer-Tropsch process

(Other cases: Agitated or non-agitated slurry reactors are less used)

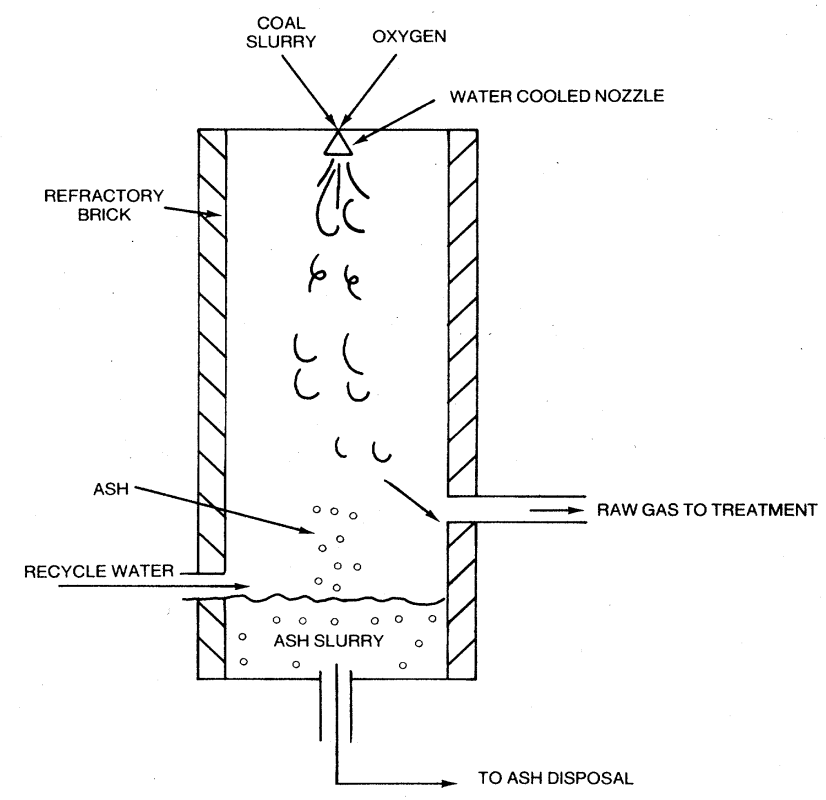

FIG. IVA-3. Entrained gasifier.

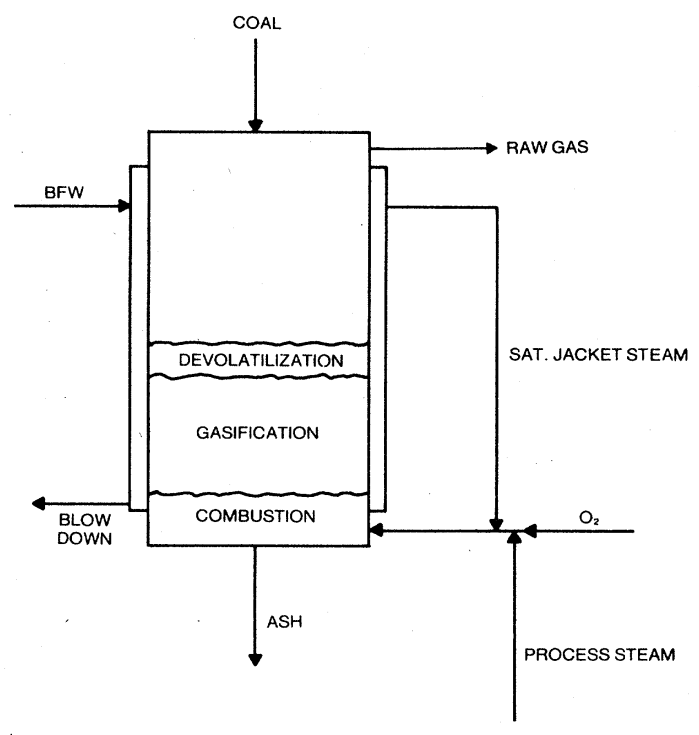

FIG. IVA-4. Moving bed gasifier.

Rev. Mod. Phys., Vol. 53, No. 4, Part II, October 1981 


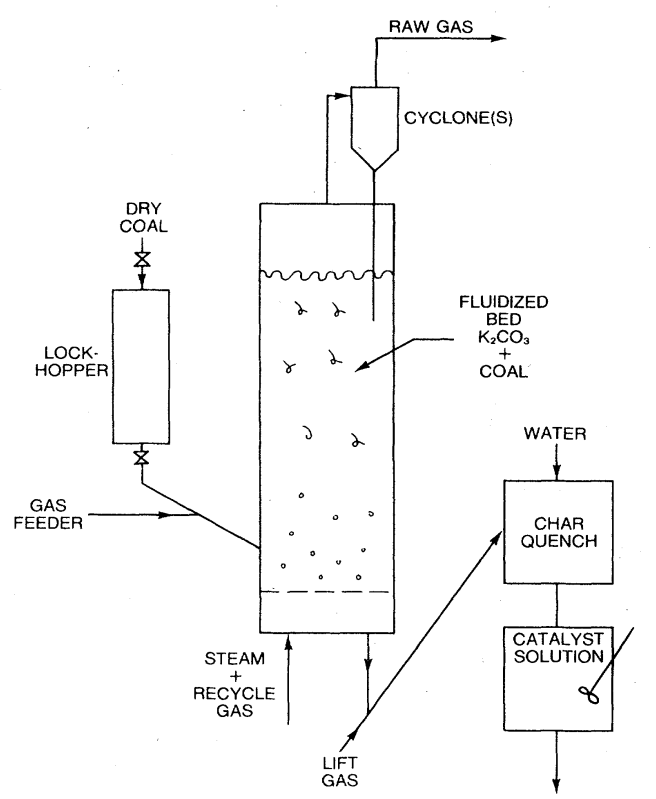

FIG. IVA-5. Fluidized bed gasifier.

severe penalty has been accepted and steam produced at a temperature of only $550 \mathrm{C}$.) One engineering advantage of the system is the use of slurry feed; coal fines and perhaps "refinery bottoms"* can be fed in this process - - a feature perceived by the FERWG review group (Penner, et al., 1980) to be very important. On the other hand the fact that the aqueous slurry brings cold water directly to the hot combustion zone lowers overall thermodynamic efficiency.

The moving bed configuration (see Fig. IV-A-4) is well exemplified by the Lurgi gasifier. Dry feed coal in sizes ranging from 1/4" to $2 "$ is lock hoppered into this reactor at the top, and the gas flow is upward or counter current. Pyrolysis takes place in the top increment of the bed and the resulting char is gasified in a mixture of steam and gaseous combustion products from the bottom of the reactor. Two advantages of this countercurrent moving bed system are: that it produces a relatively high quality gas containing significant methane and other direct pyrolysis products, and that because this product comes off at relatively low temperature ( $500 \mathrm{C})$, it is not vital to

* Heavy tar-like residual material remaining after purification of crudes. recover the sensible heat of the product gases. Disadvantages include the carcinogenic nature of the tars produced (they must be destroyed by recycling back through the gasifier), rheological problems in the bed with high swelling American coals, and poor ability to handle "fines". A related U.S. design overcomes some of these mechanical difficulties by using an extrusion feeder and mechanical stirrers within the bed (Woodmansee and Palmer, 1977).

Several fluidized bed gasifiers have been built at prototype stage. One (schematically shown in IV-A-5) uses a $15 \% \mathrm{~K}_{2} \mathrm{CO}_{3}$ catalyst to lower the reaction temperature and to achieve several other desirable improvements (Nahas and Gallagher, 1978) including a one-step production of SNG without the need to supply a pure oxygen feed.

\section{In situ (underground) gasification}

Another process currently under study and development is underground gasification which involves the same basic chemistry as the processes already described but which takes place "in situ" within the coal seam. This idea goes back to the mid-19th century and has attracted the interest of such notables as Mendeleev, Sir William Ramsey, and Lenin (Elder, 1963).

Using wells drilled at suitable locations, steam and air are delivered to the burning face of the coal seam and the resultant mixture of synthesis gas, $\mathrm{CO}_{2}$, and nitrogen returned to the surface. Neither the geometry nor the reaction parameters, such as pressure and temperature, can be as readily controlled as in conventional processes; observation and measurement are extremely difficult; and in place of materials problems ith reactor vessels one must deal with the variability and arbitrariness of geologic formations.

In situ gasification, however, shows potential for using coal deposits that are not recoverable by known mining techniques especially coal in steeply inclined seams. Environmental problems may prove to be less severe and costs lower than for more conventional techniques. The process, moreover, may prove to be more adaptable than others for really large scale utilization of coal as an energy resource. Attention of readers interested in underground gasification 
is invited to a recent extensive review article (Gregg and Edgar, 1978).

\section{Molten bath gasification}

Gasification may also be accomplished by feeding coal, steam, and oxygen (or air) into a bath of molten salt (or, in one instance, of slag) (Lowry, 1963 ; Elliott, 1981). Stoichiometrically, the end result is the same as for other gasification processes, and many impurities are removed by chemical reactions within the bath itself. There are, however, severe materials problems due to corrosion.

\section{Liquefaction, general considerations}

The overall national economy is heavily dependent upon liquid transportation fuels. Accordingly the conversion of coal to liquid fuel must be assigned a high priority.

As shown in Figure IV-A-6 (Whitehurst, 1978), coal has a much lower hydrogen content than that characteristic of high quality transportation fuels like gasoline and diesel oil. For coal the mole (atomic) ratio of hydrogen to carbon is in the neighborhood of 0.8 , but for high quality transportation fuels it is in the neighborhood of 2 . Conversion, therefore, requires that a large amount of hydrogen be reacted with the coal. In the long run, there seems little alternative but to obtain the needed hydrogen by gasification of coal.

Liquefaction processes, themselves, fall into two general classes -- direct and indirect. The approaches differ substantially. In the direct process much of the local molecular structure of the organic part of the coal (i.e. those entities that were called "sub-units" in Section III-A) is preserved in the product liquids. The coal is heated in the presence of a solvent (generally a coal derived liquid) and usually under a hydrogen atmosphere. The thermal rupture of the weaker chemical bonds in the coal gives rise to very reactive chemical intermediates (generally assumed to be free radicals). These will rapidly recombine to form refractory chars unless the broken bonds are promptly saturated or "capped" by reaction with hydrogen. The hydrogen may come directly from molecular hydrogen in the reactor atmosphere or from a "donor" organic molecule present in the solvent. Beyond the hydrogen required immediately for stabilization and prevention of char formation, some additional amount must be added to bring the hydrogen content of the liquid up to that desired for a particular transportation fuel and to remove undesirable heteroatoms, such as sulfur, nitrogen, and oxygen from the coal liquids.

In the indirect processes, the strategy is entirely different. The structure of the coal is completely destroyed at the outset by gasification. The resulting synthesis gas is a highly reactive mixture and can be rather easily converted into a variety of hydrocarbons and organic oxygenates by appropriate catalytically moderated reactions.

Direct 1 iquefaction is often argued to have better potential thermodynamic efficiency than indirect liquefaction because it preserves as much as possible of the original chemical structure of the coal. Such a conclusion is by no means inescapable, however. The net stoichiometry for the production of hydrocarbon fuels from coal and water is the same regardless of whether the indirect or direct approach is used. It can

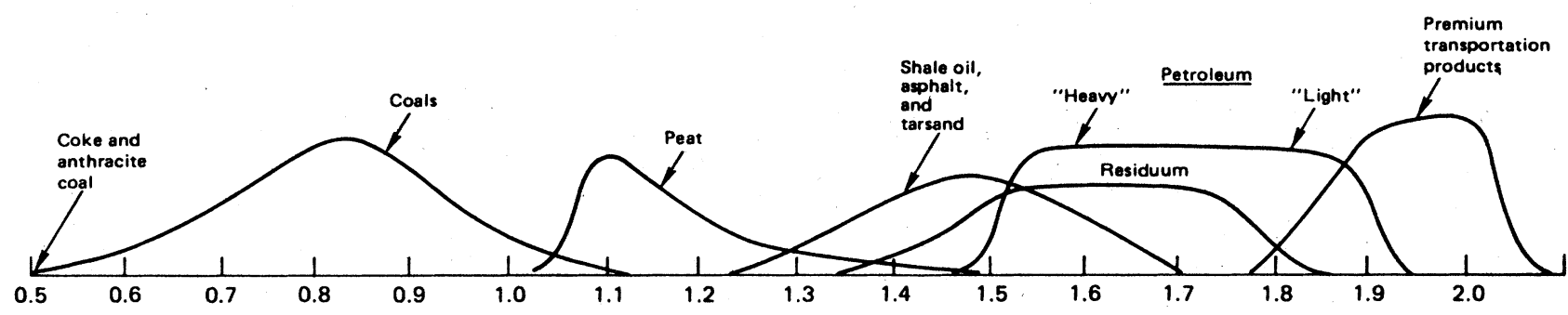

HYDROGEN/CARBON MOLE RATIO

Hydrogen/carbon ratios for various hydrocarbon sources and end products (Whitehurst, 1978)

FIG. IVA-6. Hydrogen/carbon ratios for various hydrocarbon sources and end products (Whitehurst, 1978). 
be written as shown in the following equation,

$$
(4+\beta) \mathrm{GH}_{\alpha}+2(\beta-\alpha) \mathrm{H}_{2} \mathrm{O} \rightarrow(4+\alpha) \mathrm{CH}_{\beta}+(\beta-\alpha) \mathrm{CO}_{2}
$$

where the average stoichiometry of coal is given by $\mathrm{CH}_{\alpha}$ and of the desired product by

$\mathrm{CH}_{\beta}$. The overall enthalpy of conversion is close to zero (Wei, 1981), and this suggests the possibility, at least, of a high thermodynamic conversion efficiency. But of course, the equation and the enthalpy change do not tell the whole story. With every known technology, the conversion must be carried out in several steps -- some involving high temperature, elevated pressure, and liquid and gas pumping. These steps involve some degree of irreversibility. Differences in net thermodynamic efficiency between the various routes to produce fuels of the same final composition, then, depend upon the irreversibilities and outright energy losses in heating, cooling, pressurizing, and pumping, characteristic of the particular process schemes being used, (including the efficiency of recovery and utilization of excess heat energy generated in driving the process kinetically) -- i.e. upon clever engineering and approach to ideal catalysis.

\section{a. Some important direct processes}

The principal direct processes divide largely into three classes - pyrolysis, solvent processes without addition of catalyst, and solvent processes with the addition of catalyst.

Pyrolysis, which has already been discussed, is the simplest of the direct processes; it results in an internal disproportionation of the natural hydrogen content of the coal, with the liquid fraction carrying away the lion's share of the hydrogen. Pryolysis liquids have low mineral content and a reduced sulfur level, but they tend to be unstable. Although they can be used as a boiler fuel, they have several undesirable properties in addition to their instability. Pyrolysis may be carried out under an atmosphere rich in hydrogen; this process is called hydropryolysis and produces

*Note that this conclusion is not available for the free energy change which would be enormously more difficult to estimate, and would in fact require use of information not presently available. a more stable liquid with a somewhat higher hydrogen content than that obtained in simple pyrolysis.

In the solvent process without addition of catalyst, an appropriate fraction of coal derived liquid is returned to the reaction zone. This facilitates the conversion since the returned liquid acts to some degree as a solvent for the coal. It also facilitates the transport and exchange of hydrogen so that the reactive broken bonds are hydrogenated. The process is usually carried out in a high pressure hydrogen atmosphere. Some hydrogen can react with the solvent and coal to increase the hydrogen content of the product substance. The two most important processes of this type are Solvent Refined Coal-I (SRC-I) and Solvent Refined Coal-II (SRC-II) (Suliivan, et al., 1979). They differ in the severity of the processing with the result that the SRC-II product has hydrogen content increased over that of $S R C-I$ as shown in Figure IV-A-7. Although no catalyst is added to the solvent in these processes, recent work (Granoff and Montano, 1981) has shown that mineral constituents of the coal play an important, though uncontrolled, catalytic role in the hydrogenation and hydrogen transfer.

The catalytic activity of the mineral content varies from coal to coal and is weaker than desired. Additional catalytic material, therefore, is added in many of the solvent processes. Solvent molecules must be chosen for their ability to transfer hydrogen to the dissolving coal and, subsequently, to be themselves rehydrogenated under the influence of the catalyst being used.

Two variations which combine the use of an added catalyst and this kind of transfer mechanism are possible -- the solvent can be rehydrogenated externally to the vessel in which the coal is dissolving; or the catalyst can be present in the reaction chamber together with the coal and the solvent. One process of each type is now under development in the U.S.

In the Exxon Donor Solvent (EDS) process (Fant, 1978; Epperly, 1978, 1979), a hydrogen transfer solvent derived from the product liquids is added to the coal as in the SRC processes. The product liquids are catalytically rehydrogentated in a separate reactor before being fractionated and (in part) recycled to the main liquefier. A simplified flow diagram of the EDS process is given in Figure IV-A-8.

In the H-Coal process (Stein, et al., 


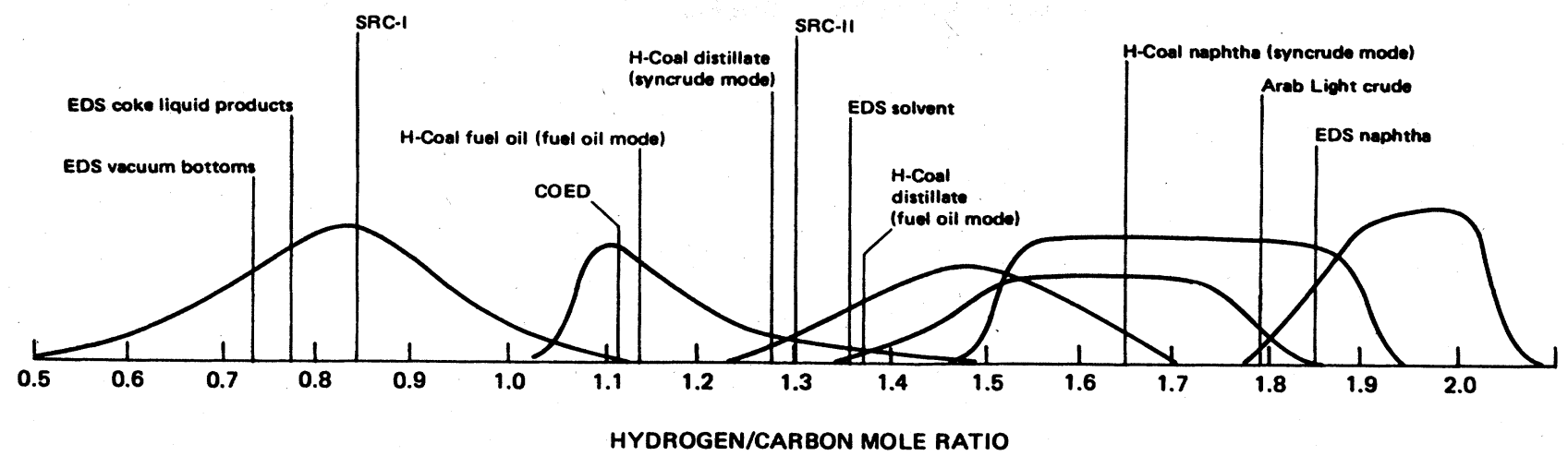

Hydrogen/carbon ratios for products of coal 11quefaction pilot plants, with Arab Light crude for comparison (adapted from Whitehurst, 1978)

FIG. IVA-7. Hydrogen/carbon ratios for products of coal liquefaction pilot plants, and Arab Light crude for comparison (adapted from Whitehurst, 1978).

1977, 1978; Hydrocarbon Research, 1978) catalyst, coal, and solvent are mixed and simultaneously present in the reactor so that the solvent is continuously rehydrogenated in the presence of the dissolving coal. A flow diagram of the process is given in Figure IV-A-9. The coal, solvent (recycle slurry oil), and hydrogen are reacted in an ebullating-bed reactor. The material in the reactor is maintained in a fluidized ebullating state by means of a pump which recirculates the liquids. Temperature of the order of 470C and a presssure of about 3000 psi are used. When the products are

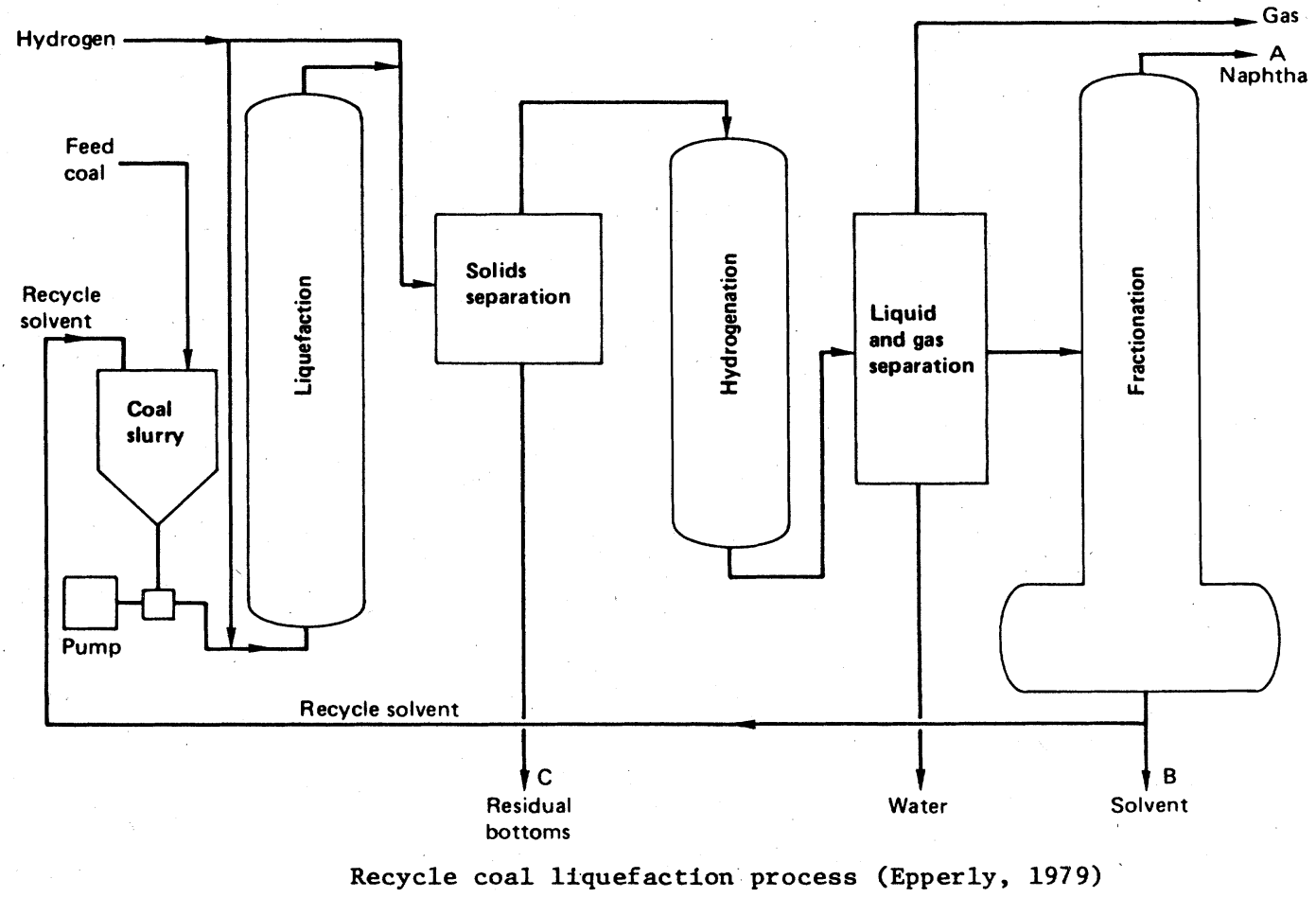

FIG. IVA-8. Flow diagram of the EDS pilot plant unit (Epperly, 1979). 


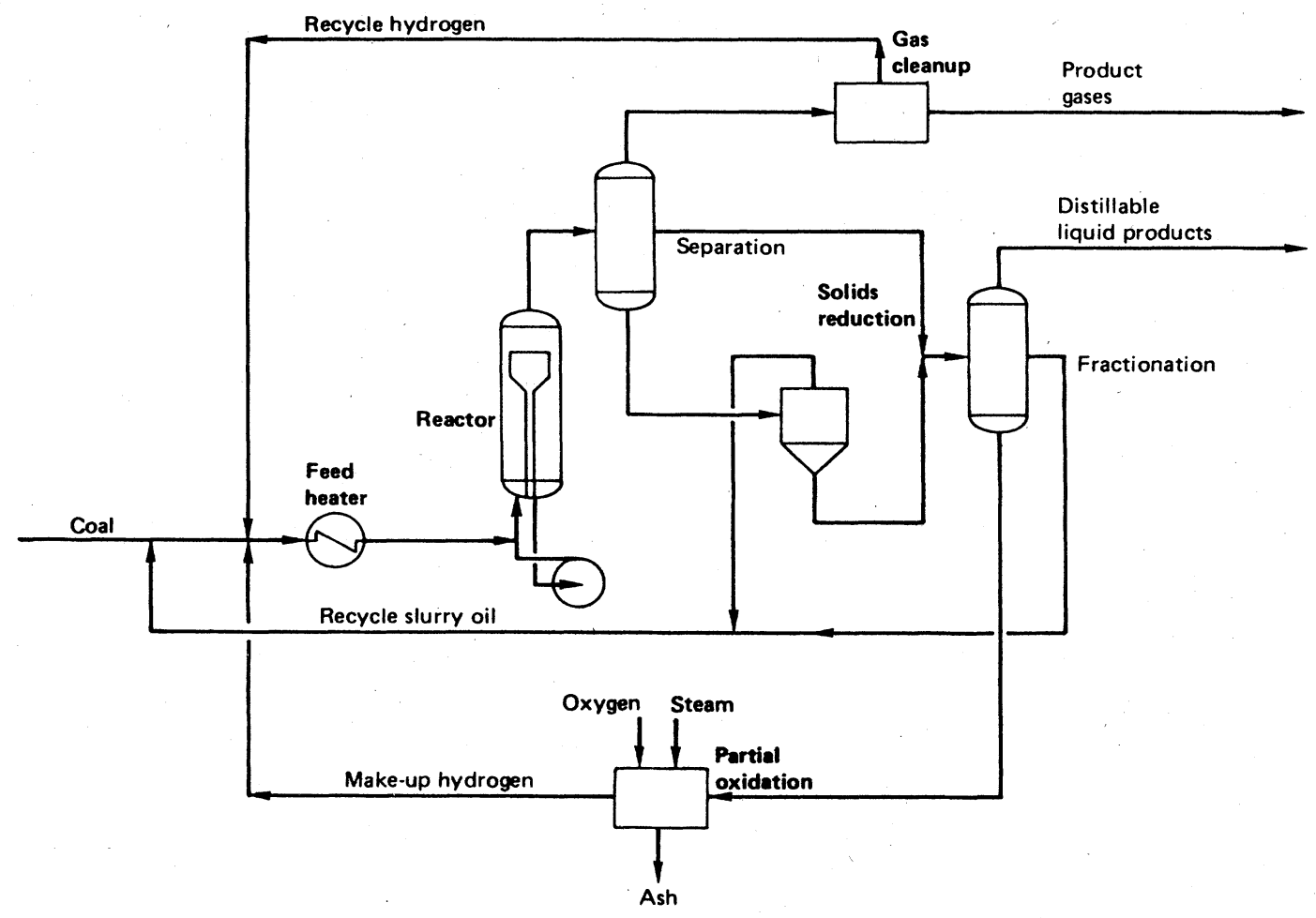

H-Coal 1iquefaction process (Hydrocarbon Research, 1978)

FIG. IVA-9. Flow diagram of $H$-Coal process (Hydrocarbon Research, 1978).

subsequently fractionated, material with boiling point above 510C, the ash, and any remaining unconverted coal are sent to a gasifier to produce hydrogen needed for the process. The H-Coal reactor is rather complex and presents several interesting problems in multiphase flow; a diagram is given in Figure IV-A-10.

Early rationales for the two processes were: in the EDS process, that the separate hydrogenation of the solvent permits a more active and/or expensive catalyst to be used since it need not be mixed with ash and coal particles; and, in the case of the H-Coal process, on the other hand, that an intimate mixture of catalyst, coal, and coal liquids presents a much wider spectrum of molecules on which the catalyst can act.

\section{b. Indirect processes}

The synthesis gas produced by a gasifier has higher free energy content than its stoichiometric equivalent of hydrocarbons plus $\mathrm{CO}_{2}$ and $\mathrm{H}_{2} \mathrm{O}$. Thus, it is easy to produce hydrocarbon's, oxygenated organic compounds, $\mathrm{CO}_{2}$, and $\mathrm{H}_{2} \mathrm{O}$ from synthesis gas by a variety of ${ }^{2}$ catalytic processes with the release of considerable heat. The ultimate efficiency of any system incorporating these processes depends on the effective utilization of this heat. There are two general routes that have been commercialized for the conversion of syngas into high quality transportation fuels -- the Fischer-Tropsch synthesis and the Methanol route.

In the Fischer-Tropsch process (Hoogendoorn, 1973), as presently used by SASOL in South Africa, the synthesis gas is converted to a broad spectrum of hydrocarbons and oxygenates over an iron catalyst in either a fixed bed or an entrained fluidized bed. The hydrocarbons and oxygenates are primarily straight chained organic molecules with few ring structures. The molecular weight range can be quite large, and in the fixed bed substantial quantitites of wax may be 
produced. Because of the straight chain nature of the Fischer-Tropsch product, very good diesel fuels are readily made, but considerable isomerization is required to make good gasolines. This situation is improving, however, with the introduction of new shape selective zeolite catalysts (See Section IV-F) that are capable of modifying the initial Fischer-Tropsch products to produce aromatics and highly branched paraffins.

Considerable efforts are in progress to carry out Fischer-Tropsch synthesis in slurry reactors that are able to use synthesis gases with low (unshifted) $\mathrm{H}_{2} / \mathrm{CO}$ ratios (0.5-0.7) (Kuo, et al., 1978). Thls low ratio synthesis gas can be produced by gasifiers that offer considerable advantage in cost and efficiency. Indeed, for this reason such slurry (i.e. liquid state) reactors seem to offer the most immediate promise for arriving at an economically viable synthetic liquid fuel technology. Processes are being developed for conversion of these products, with high yield (of the order $80 \%$ ), into high octane gasolines over zeolite catalysts. The flow conditions in slurry reactors offer interesting

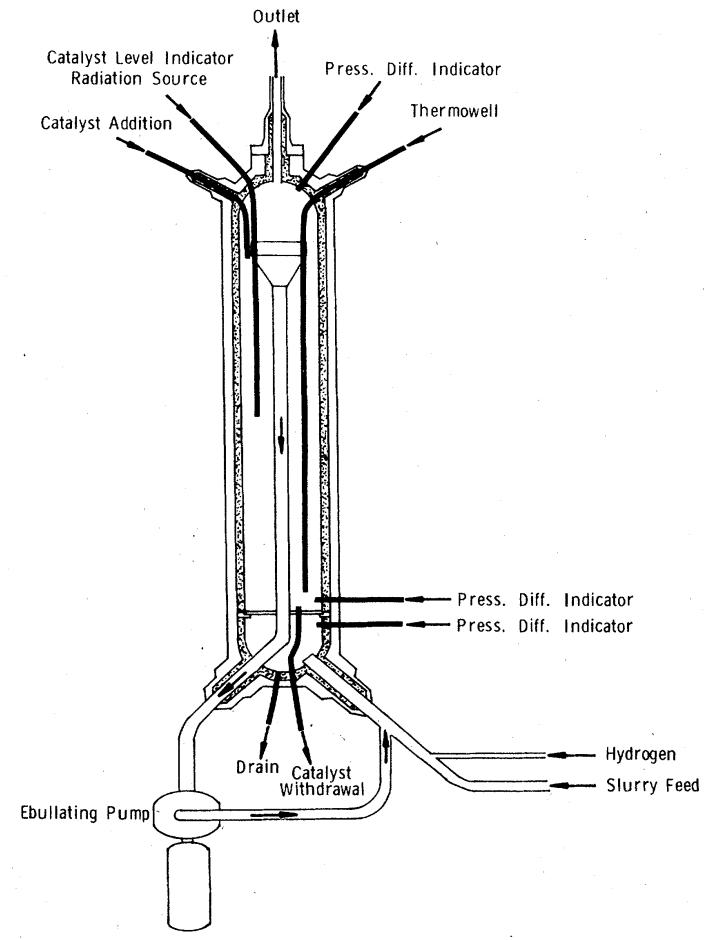

FIG. IVA-10. Ebullating bed reactor (Hydrocarbon Research, 1978). possibilities for multiphase flow studies by physicists, studies which might contribute significantly to the further development and improvement of these processes.

The other important indirect route begins with conversion of synthesis gas into methanol (Schreiner, 1978). A fixed bed reactor is usually used with a $\mathrm{Cr}-\mathrm{Zn}-\mathrm{Cu}$ catalyst. This is a well established commercial process. Methanol itself can be used as a high quality transportation fuel. Beyond this, the recent development of processes for converting methanol into high octane gasoline over a zeolite catalyst will increase the importance of the methanol synthesis in the production of transportation fuels. (Meisel, et al., 1976). Reactors for conversion of methanol to gasoline are being developed in both fixed and fluid bed configurations. Also, in current developments in slurry reactors for Fischer-Tropsch synthesis (discussed in the preceding paragraph), the catalysts can be supported on zeolites, so as to incorporate shape selective features into a single reactor.

\section{Direct combustion}

\section{a. Pulverized coal combustion (PCC)}

By far the most highly developed and widely practiced of the energy technologies relating to coal -- at present far outweighing all others in economic importance -- is direct combustion in the boilers of electric power stations. This pulverized coal combustion (PCC) technology has benefited from several generations of incremental improvement based upon operating experience at the full scale industrial level and currently is the object of increasingly systematic scientific study.

The amount of coal burned in a large power station (10,000 tons per day for a 1000 MW plant) is costly enough to provide a very strong incentive toward higher efficiency. And indeed, just prior to the advent of environmentally motivated emission control regulations, the overall efficiency of a base-loaded state-of-the-art plant had reached approximately $42 \%$, expressed as the fraction of the enthalpy of the input coal that appears as electrical energy at the output.

A new technical challenge was posed by the imposition of emission controls, and much research effort has been expended in recent years to develop economical systems for 
removal of particulate matter and $\mathrm{SO}_{2}$ from the flue gas of coal fired power stations. Use of the best methods known at present entails a reduction of several percent in overall plant efficiency. No commercial method of removing nitrogen oxides from stack gas has yet been devised; measures for reduction of $\mathrm{NO}_{\mathrm{X}}$ emissions therefore, focus upon controlling the combustion regime to reduce $\mathrm{NO}_{\mathrm{x}}$ formation from the start.

One major object of investigation is the possibility of retrofitting existing oil-fired plants to burn powdered coal or, more probably, a coal-oil slurry (See Sec. IV-E); this effort encounters major problems with handling the ash which may be 100 times as much as the plant was originally designed to tolerate.

In a modern coal-fired boiler the coal is finely pulverized ( 50 micron median), entrained in part of the combustion air, and fed with additional air into a very large combustion chamber through burners arrayed around the walls. See Figure IV-A-11. The combustion chamber may be of the order of 150 feet high, and the burners can be as many as 50 in number. The upper furnace walls are lined with water tubes.

The stable flame produced in the jet of gas and suspended coal particles reaches a temperature of about $2000 \mathrm{~K}$. Particle residence times in the furnace are of the order of 0.1 to 2 seconds. As they $r$ ise through the upper furnace hot combustion products cool sufficiently by radiation to the water tube walls so that the mineral matter (ash) re-solidifies and can be carried through the boiler passages without plugging or "fouling". The time-temperature-distance profile for coal particles in a $500 \mathrm{MW}$ utility boiler is shown in Fig. IV-A-12.

The flue gases, before being permitted to exit from the stack, must be treated to remove ash particulates (at present, this is usually done with electrostatic precipitators and/or fabric filters in "bag houses") and sulfur

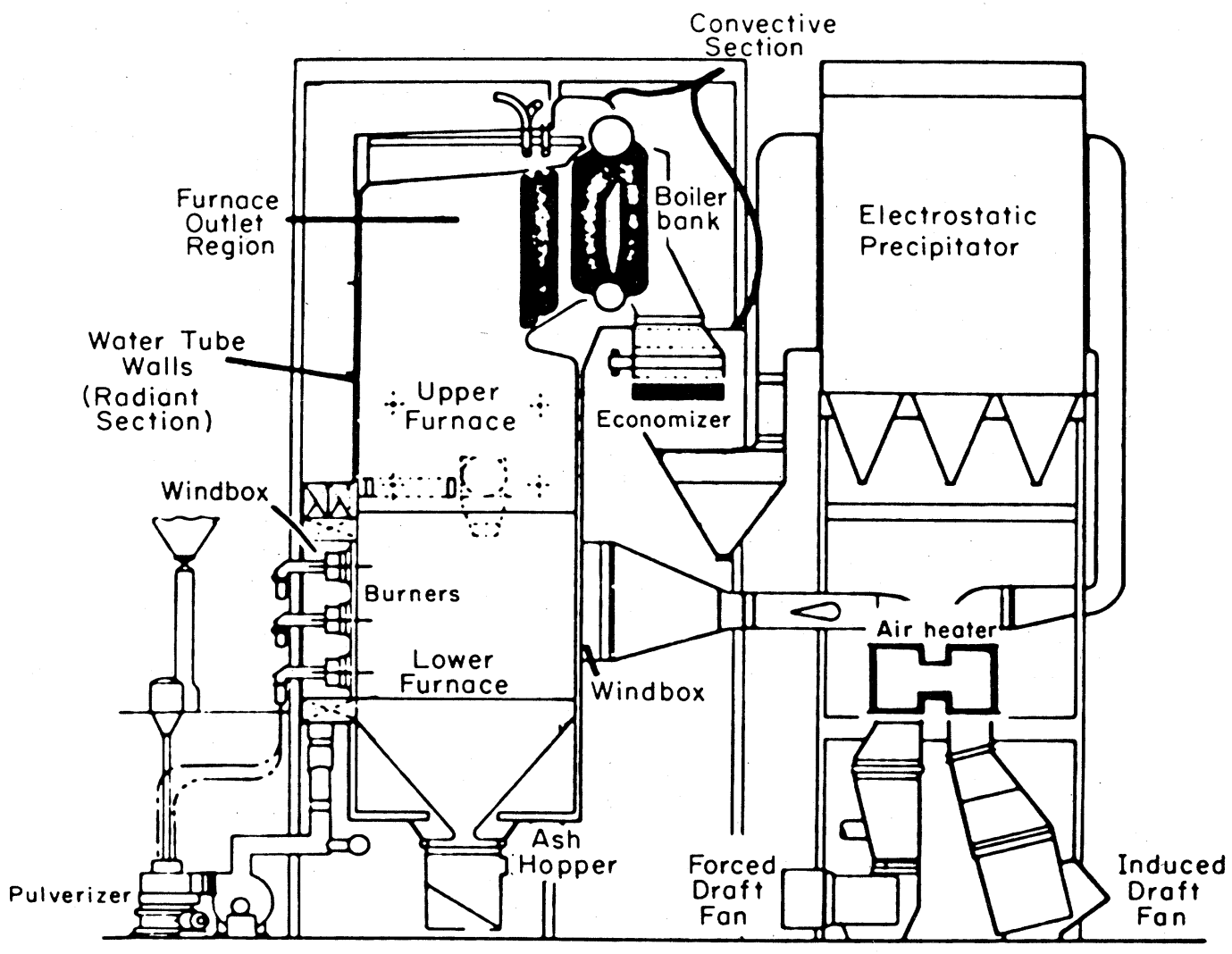

FIG. IVA-11. Schematic of pulverized coal-fired electric utility boiler (Hardesty and Pohl, 1979). 


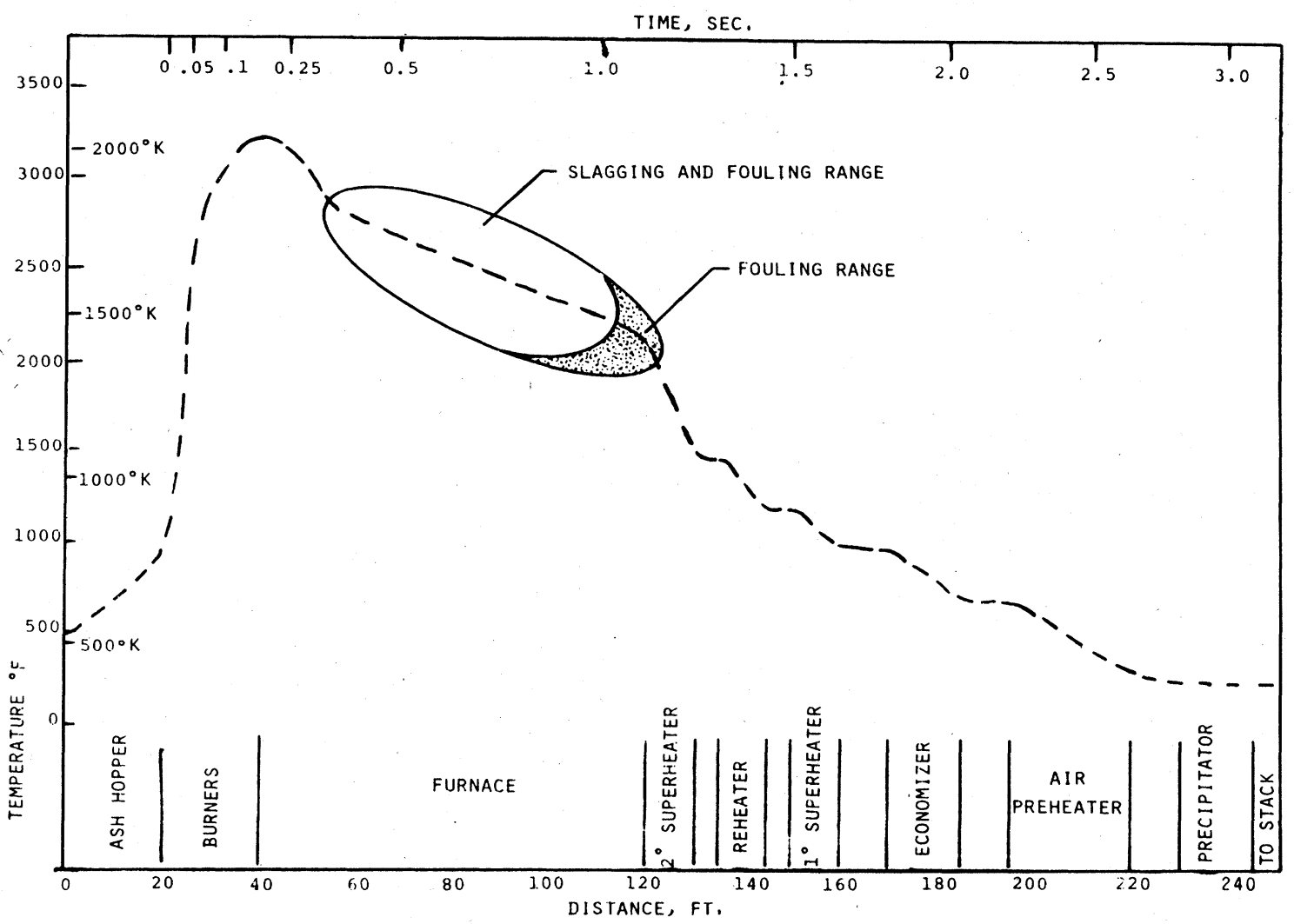

FIG. IVA-12. Time-temperature-distance profile in a $500 \mathrm{MW}$ coal-fired utility boiler (regimes of slagging and fouling are crosshatched) (Hardesty and Poh1, 1979).

dioxide. The $\mathrm{SO}_{2}$ removal techniques currently most advanced in development are "scrubbers" of one kind or another in which the flue gases are treated with limestone sprays.

All of these treatments, of course, consume significant amounts of energy (requiring blowers and sometimes even re-heating of the flue gas, to maintain furnace draft) and with a consequent lowering of overall plant efficiency.

The physics of filtration is very imperfectly understood and may represent an underexploited area of research opportunity (Billings and Wilder, 1970). In particular, electrostatic forces can play an important role because of their strength relative to gravitational, thermal, or adhesive forces for particles in the 0.1 to 1 micron range. A potentially important, unexplained observation is that the filtration of electrically charged particles can proceed with only a slight increase in pressure differences due to filter loading. No adequate model for the process exists (Lamb and Kostanza, 1980; Penny, 1977. 1978).

Formation of nitrogen oxides is very sensitive to the combustion regime (in different ways for NO arising from atmospheric $\mathrm{N}_{2}$ and from fuel-bound nitrogen); a single incorrectly adjusted burner can account for a large share of the $\mathrm{NO}_{\mathrm{x}}$ emissions from a boiler. (See Section IV C) For this and other reasons it is desirable to control the burners individually, and there is need for instrumentation to monitor flame conditions directly. This is discussed in Sections IV B and IV C.

\section{b. Fluidized bed combustion}

A potential alternative to the highly developed and widely used PCC boiler may be offered by direct combustion of coal in 
fluidized beds -- a new technology which is currently the object of much research and development effort. In this system crushed coal is added to a fluidized bed of crushed limestone or dolomite. Air for combustion enters from below through plenum chambers. The rate of air feed is adjusted so that aerodynamic forces just neutralize gravitational ones for the crushed solids; the individual granules become very mobile; and the bed exhibits some properties of a liquid. Since steam tubes can be situated within the fluidized bed where thermal transfer is facilitated, the maximum temperatures can be kept below those in a conventional boiler (where initial heat transfer is radiative) and thermal $\mathrm{NO}_{\mathrm{x}}$ emissions should be more easily controlled. SO is removed by chemical reaction with the bed as soon as it is liberated. (Generally, the amount of air needed to fluidize the bed exceeds that which is useful for combustion and this lowers overall thermal efficiency.)

\section{Some advanced concepts embodying combined cycles}

\section{a. Pressurized fluidized bed combined cycle}

A more advanced direct combustion concept is that of the "pressurized fluidized bed combined cycle" (Corman and Fox, 1976). Fluidized bed combustion, in this case, is carried out at elevated pressure; this brings advantages but also requires substantial energy for the pressurization. Energy is extracted from the high pressure/high temperature combustion products in two steps. First they are "let down" through a gas turbine which recovers enough energy to drive both the compressor and an auxilliary electric generator. Exhaust from the gas turbine is still at a high temperature and is used to raise steam for a conventional turbine. This system furnishes an example of "combined cycle" operation -- in which high and low temperature heat engines are cascaded so as to better utilize heat from a high temperature source and to realize a greater overall efficiency than practical engineering considerations will allow in a single cycle.

A major technical problem here -- and with several other combined cycle concepts for coal utilization - - is that of "hot gas clean-up". Coal combustion and gasification products are laden with ash and corrosive substances (e.g. alkali metal and vanadium compounds) which must be removed or reduced to insignificant levels before being fed into "topping cycles" such as gas turbines or fuel cells. The need to carry out the clean-up without excessive loss of pressure or temperature raises a number of difficult engineering and materials problems. Some of these are discussed in Section IV D.

\section{b. Magneto-hydrodynamic conversion "MHD"}

MHD conversion (Petrick and Yashumyatsky, 1978; Jackson, 1977) is straightforward in basic concept and in its basic device configuration; it does not require highly stressed moving parts, nor any solid parts at all which are not accessible for external cooling; and it does not demand the maintenance of close dimensional tolerances. For these reasons it is thought possible to build an MHD generator to endure gas conditions far more severe than those permissible in a conventional gas turbine, and such a generator should have the potential both for increased power handling capability and increased reliability. Used as the high temperature end of a combined cycle, MHD should afford the improved efficiency associated with a higher Carnot factor. To realize these hoped for advantages in practice, however, will require the solution of unusual and formidable engineering and materials problems. Some of the latter are discussed in Section IV D.

In an MHD generator the extremely hot gases resulting from direct combustion of coal (with preheated air) are seeded with potassium or other easily ionized substances to make them electrically conductive. They are then expanded in a very high speed nozzle or channel. See figure IV-A-13. The channel is situated in an intense transverse magnetic field ( 6 Tesla from a superconducting coil has been proposed). Being a high speed moving conductor in a transverse magnetic field, the stream of (partly) ionized combustion products becomes the site of an induced electromotive force, and electrical power can be taken off through a suitable set of electrodes on the channel walls. Combustion products exiting from the channel are still at extremely high temperature and are used first to heat the incoming air for combustion and subsequently 
to raise steam for a conventional steam turbine. It is necessary at some point to recover for reuse as much as possible of the seed substance.

Since the inlet temperatures to the MHD channel may be as high as $2400 \mathrm{C}$ and slag is present, the corrosion and erosion problems are severe. Besides contending with these, the channel design must meet quite exacting electrical requirements. As indicated in figure IV-A-13, it has been proposed to meet these simultaneous requirements by resort to laminated and composite structures of metals, insulators, and refractories.

\section{c. High temperature fuel cells}

A promising approach to improved efficiency in coal utilization is the development of fuel cells which can operate on hydrogen or synthesis gas, and the incorporation of these into combined cycle or co-generation systems. It was already noted (in the discussion of indirect liquefaction processes) that the free energy content of synthesis gas is higher than that of the synthetic liquid fuels into which it can readily be converted. One way to conserve this free energy is to use synthesis gas -- or hydrogen obtained from it by shift reaction -- as feed for suitably designed fuel cells. The fuel cell mechanism is electrochemical rather than thermal, so the efficiency need not be limited by a Carnot factor. Conversion efficiency, moreover, should be relatively insensitive to plant size or load level, so that some kinds of fuel cell systems should be highly adaptable for modular and decentralized operation.

Two kinds of fuel cells are currently the objects of substantial research and development effort -- phosphoric acid cells and molten alkali carbonate cells. Phosphoric acid cells are far advanced in development, to the extent that a $4.8 \mathrm{MW}$ pre-prototype unit in a urban setting is under actual construction. Phosphoric acid celis use carbon electrodes impregnated with finely dispersed platinum catalyst. They consume only hydrogen and, in fact, can be adversely affected by carbon monoxide; synthesis gas must, therefore, be shifted and purified for use in phosphoric acid cells. Their operating temperature is about $190 \mathrm{C}$ which means the rejected heat is convenient for co-generation of process steam and low grade heat for space conditioning. Phosphoric acid cells are somewhat tolerant of sulfur, but there are numerous problems relating to catalyst de-activation by sintering, corrosion of carbon electrodes, and mechanism of oxygen reduction at the

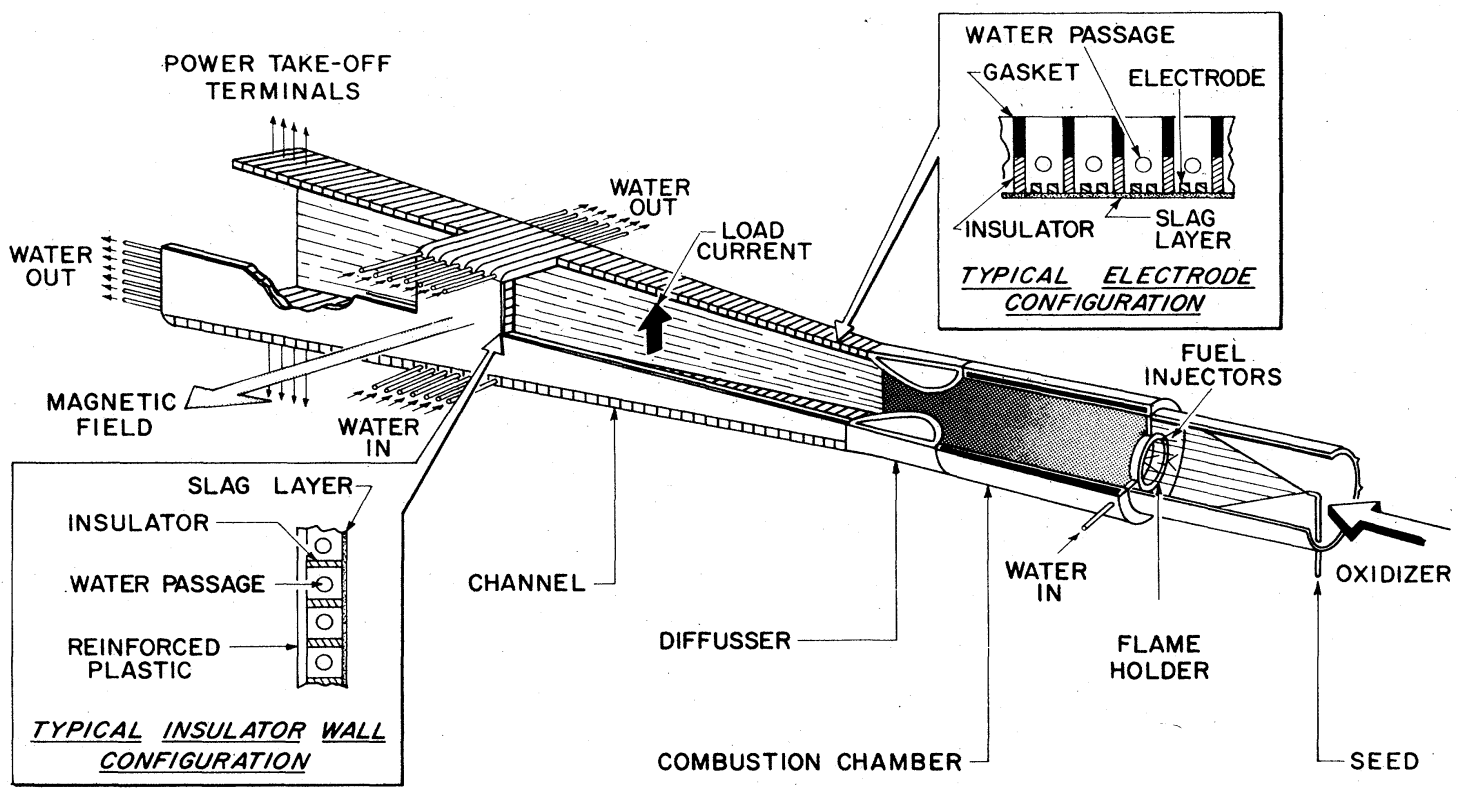

FIG. IVA-13. Schematic MHD channel. 
(relatively inefficient) cathode.

Molten carbonate cells operate at much higher temperatures (approximately $650 \mathrm{C}$ ). They are, therefore, suitable for integration with conventional steam turbine bottoming cycles in combined cycle configurations promising quite high overall efficiencies (50\% is thought possible). These cells are capable of utilizing the carbon monoxide content of synthesis gas but at present are quite sensitive to sulfur poisoning. Thermodynamically, it would be attractive to feed the high temperature product of a coal gasifier through a hot gas clean-up stage and thence directly to molten carbonate fuel cells. At present, however, hot gas clean-up technology has not advanced to a level which would permit this -- nor can molten carbonate cells tolerate dirty gas. Even more serious are problems relating to the service life of molten carbonate fuel cell components and trade-offs between lifetime, efficiency, and cost.

For further information the interested reader may find it useful to consult: Fickett, 1978; National Fuel Cell Seminar, 1980; and Browall and Mazandarany, 1980.

\section{Mining}

Coal technology begins with mining which currently is classified into underground mining and surface (open cast or strip) mining. Primary concerns here are safety, efficiency, and the environmental effects. The technical challenges lie mostly in the provinces of mining engineering, civil and mechanical engineering, operations analysis, and industrial planning. The Study Group devoted relatively little attention to mining but did note several problems that might be solved by improved physical instrumentation. Well known, of course, is the need to detect and monitor toxic and explosive gases. Other examples may be found in the need of a "continuous mining" machine to detect large inclusions of shale or other mineral matter within the coal seam and to sense the exact location of the coal-shale interface at the upper or lower boundary of the seam. The reader interested in mining and preparation of coal can find a general survey in the Keystone Coal Industry Manual compiled by Mining Information Services and published by McGraw-Hill.

\section{Coal preparation}

Coal -- after it is mined and before it is burned, coked or fed into gasifiers -generally receives some preparatory treatment. Grinding, sizing, and washing are common. Flotation methods are used to process about $40 \%$ of the coal production. These depend upon specific gravity differences or wettability differences between mineral matter and the carbonaceous components of the coal as mined.

Some treatments are designed to reduce unwanted mineral matter, especially inorganic sulfur associated with the ash. Of particular interest is high-gradient magnetic separation for coal desulfurization (Maxwell, 1978). (Also see Section IV-E.) Others are intended to control caking behavior, etc. Sometimes coals are blended to reduce variability and bring them to some reduced sulfur content or to achieve a better coke by combining and averaging the peculiar characteristics of several coals.

For some processes it would be advantageous to reduce coal to extremely fine particles (a few microns) if that could be done economically. The Study Group gave considerable attention to this question of "comminution" or grinding, and it is discussed in Section IV-E of this report.

\section{References}

Anthony, D. B. and J. B. Howard, 1976, AIChE J., 22, 1976, 625.

Billings, C. E. and J. Wilder, 1970 , Handbook of Fabric Filter Technology, GCA Inc., Bedford, MA.

Browall, K. W. and Farhad N. Mazandarany, 1980, Development of Molten Carbonate Fuel Cells for Power Generation, 15th Intersociety. Energy Conversion Engineering Conference, Seattle, Washington, August 18-20, 1980 . (Available from General Electric Corp. Research and Development as Reprint 非905.)

Carberry, J. J., 1976, Chemical and Catalytic Reaction Engineering, McGraw-Hill, New York.

Davidson, J. F. and D. Harrison, Editors, 1971, Fluidization, Academic Press, London.

Elder, J., 1963 , "The Underground Gasification of Coal," Chemistry of Coal Utilization, H. H. Lowry, Ed., 
Supplementary Vol. 3, John Wiley Sons, Inc., New York, pg. 1023.

Elliott, M. A., 1981, Chemistry of Coal Utilization, and Supplement, John Wiley Sons, New York.

Epperly, W. R., 1978, EDS Coal Liquefaction Process Development, Phase 3-B/4. Annual Technical Progress Report. Washington, D.C.: U.S. Department of Energy (FE-2873-17).

Epperly, W. R., 1979, EDS Coal Liquefaction Process Development, Phase 4. Annual Technical Progress Report. Washington, D.C.: U.S. Department of Energy (FE-2983-35).

Fant, B. T., 1978, EDS -- Coal Liquefaction Process Development, Phase 3A. Final Technical Report, Vol 3. Washington, D.C.: U.S. Department of Energy (FE-2353-20).

Fickett, Arnold, 1978, "Fuel Cell Power Plants." Scientific American, December, 1978, p. 70 .

Fumich, George, Jr., 1980, Statement of the Assistant Secretary for Fossil Energy, U.S. Department of Energy, before the Subcommittee on Interior and Related Agencies of the Committee on Appropriations, U.S. Senate, May 8, 1980.

Gluskoter, J. S., 1975, Adv. Chem. Ser., $141,1$.

Granoff, B. and P. A. Montano, 1981, pp. 291:398 in Chemistry and Physics of Coal Utilization -1980, A IP Conference Proceedings No. 70 , edited by B. R. Cooper and L. Petrakis.

Gregg, D. W. and T. F. Edgar, 1978, "Underground Coal Gasification," AIChE J., 24, 753.

Hardesty, D. R. and J. H. Poh1, 1979, Sandia Laboratories Report 78-8804.

Hoogendoorn, J.D., 1973, Experience with Fischer-Tropsch Synthesis at Sasol. Clean Fuels from Coal Symposium Papers, pp. 353-365. Chicago, Ill.: Institute of Gas Technology ( CFFC-1).

Hydrocarbon Research, Inc., 1978, H-Coal Integrated Pilot Plant. Final Report, Vol. 1. Electric Power Research Insitutue. Palo Alto, Calif.: Research Report Center (EPRI-AF-681, Research Project 238-1).

Jackson, William D., 1978, MHD Electrical Power Generation + Program Status Report. Scientific Problems of Coal Utilization, ed. by B. R. Cooper. Technical Informtion Center, U.S. Dept. of Energy, p. 113.

Jensen, G. A., 1975, Ind. Eng. Chem. Proc. Dev., 14, 308.

Kunii, $\bar{D}$. and 0 . Levenspiel, 1969 , Fluidization Engineering, John Wiley Sons, Inc., New York.

Kuo, J. C. W., R. Shinnar, J. Wei, and C. T. D. Wu, 1978, Gasifier Study for Mobil Coal to Gasoline Processes. Final Report. Washington, D.C.: U.S. Department of Energy (FE-2766-13).

Lamb, G. E. R., and P. A. Kostanza, 1980, Textile Res. J. 50, 661 .

Lowry, H. H., $19 \overline{63}$, Chemistry of Coal Utilization, Supplement. John Wiley Sons, New York.

Maxwel1, E., 1978, pp. 90-112 in Scientific Problems of Coal Utilization, Edited by B. R. Cooper, Technical Information Center, U.S. Dept. of Energy (available as CONF - 770509 from National Technical Information Service, Springfield, VA 22161 ).

McKee, D. W., 1974, Carbon, 12, 453.

McKee, D. W., 1979, Chemistry and Physics of Carbon, pg. 16, Marcel Dekker, New York, 1979.

Meisel, S. L., J. P. McCullough, C. H. Lechthaler, and P. B. Weisz, 1976 , ChemTech., 6, 86-89.

Mining Information Services. Keystone Coal Industry Manual. McGraw-Hill, New York.

Nahas, N. C. and S. E. Gallagher, Jr., 1978, 13th IECED, 3, 2143.

National Fuel Seminar Abstracts, July 14-16, 1980, San Diego, Ca. (Copies available DOE and EPRI)

National Academy of Sciences/National Research Council, 1977, Assessment of Low - and Intermediate-BTU Gasification of Coal, Report FE/1216-4 available from National Technical Information Service.

Penner, S. B. (Chairman, Fossil Energy Research Working Group, FERWG), 1980 , "Assessment of Long-Term Research Needs for Coal-Liquefaction Technologies," DOE Contract DE-AC01-79E R 10007.

Penney, G. W., 1977, Powder Separation 18, 111 .

Penney, G. W., 1978, "Electrostatic Effects in Fabric Filtration", EPA Publ. 非PA-600/7-78-1 42a.

Petrick, M. and B. Yashumyatsky, 1978, Open-Cycle Magnetohydrodynamic Electric Power Generation, edited by M. Petrick and B. Yashumtatsky. Argonne National Laboratory Technical Report DOE-PR-119. 
Schreiner, M. 1978, Research Guidance Studies to Assess Gasoline from Coal by Methanol-to-Gasoline and Sasol-Type Fischer-Tropsch Technologies. Washington, D.C.: U.S. Department of Energy (EF-2447-13).

Smith, J. M., 1970, Chemical Engineering Kinetics, McGraw-Hill, New York.

Solomon, P. R. and M. B. Colket, 1977, "Coal Devolatilization," l6th International Symposium on Combustion, pg. 131.

Stein, T. R., J. G. Bendoraitis, A. V. Cabal, R. B. Callen, M J. Dabkowski, R. $\mathrm{H}$. Heck, H. R. Ireland, and C. A. Simpson, 1977, Upgrading of Coal Liquids for Use as Power Generation Fuels. Electric Power Research Institute. Palo Alto, Calif.: Research Report Center (EPRI-AF-444, Research Project 361-2).

Stein, T. R., A. V. Cabal, R. B. Callen, M. J. Dabkowski, R. H. Heck, C. A. Simpson, and S. S. Shih, 1978, Upgrading of Coal
Liquids for Use as Power Generation Fuels. Electric Power Research Institute. Palo Alto, Calif.: Research Report Center (EPRI-AF-873).

Sullivan, R. F., B. E. Stangeland, and H. A. Frumkin, 1979, Refining of SRC-I and SRC-II. Paper presented at Refining of Syncrudes Contractors Conference, Richmond, Calif., May 7-8, 1979, sponsored by U.S. Department of Energy.

Wei, J., 1981, Ind. Eng. Chem. Proceedings (to be published).

Whitehurst, D. D., 1978, American Chemical Society Symposium 71: Organic Chemistry of Coal, pp. 1-35. Washington, D.C.: American Chemical Society.

Woodmansee, D. E. and P. M. Palmer, 1977, ACS Symposium on Coal Gasification Kinetics, 22 (1), No. 1, 158.

Yoon, H., J. Wei, and M. M. Denn, 1978 , AIChE J., 24, 885 . 
Research for the Technologies

\section{B. INSTRUMENTATION AND CONTROL}

\section{Introduction}

Coal utilization technology stands in urgent need of improved instrumention -- a need rooted in considerations both of process control and of scientific understanding.

Process control requires continuous real-time or near real-time measurement or "monitoring" whereas scientific understanding is as well served by measurements yielding time-dependent, but not instantaneous, data. Controls of coal processing plants must be adequate to insure: safety, reliability, "compliance" (with environmental regulations), economy and efficiency, and adaptability to variations of output demand and feed-coal properties. Requirements in each of these categories are very exacting. Safety, for example, must be assured for plants which process enormous tonnages of highly reactive materials at pressures up to several hundred atmospheres and temperatures up to $2000 \mathrm{~K}$; the potential for fires or explosions endangering personnel and causing damage to major capital facilities is ever present. Likewise, "compliance" may require better than $99 \%$ effective removal from plant effluents of naturally arising pollutants.

Scientific understanding, which is prerequisite to sophisticated plant control, has greater need for precision and completeness and less need for instrumental durability and immediate availability of results than is the case for practical process control. The minimum scientific goal must be a level of understanding sufficient to make good dynamic models -- models reliable both for scale-up of pilot plant processes and for the design and computer assisted testing of control systems. Present knowledge does not suffice for successful modeling of the responses of existing pilot plant facilities even within the physically attainable and observed range of operating conditions -- and a practical control system must be trustworthy in serious off-normal excursions as well as in start up, shut down, and steady operating situations.

Dynamic models, which are based on data from diagnostic instrumentation, make possible a more intelligent choice of instruments for process control by quantifying requirements on these instruments. In addition, the information gained from the models may ease measurement problems, by permitting the use of instruments (which may be already available or at least easier to develop) to measure other process parameters related (through the model) to the parameter to be controlled.

A measurement intensive and modeling intensive approach to process improvement and scale-up appears necessary if the desired timetable for coal technology development is to be realized. Precedents do exist for the success of a trial and error approach to industrial process chemistry, even for processes (e.g., oil refinery catalytic crackers) with severe operating conditions. These successes, however, resulted from development extending over several decades at a time when energy was cheap and computers unavailable. Now energy is expensive, construction costs are astronomical, and the projected need is for coal utilization processes operating on a large scale and in large numbers within ten or fifteen years. Mistakes should not be cast in concrete and steel where there is any chance that testing of processes and their control can be done more quickly and cheaply with the aid of computers.

\section{a. General nature of the difficulty}

Instrumentation of coal processes presents unusual difficulties. Advanced processes - liquefaction, gasification, f 1 uidized-bed combustion, and magnetohydrodynamics - - involve the transformation of large amounts of solid coal (crushed to median sizes as small as $40 \mu \mathrm{m}$ ) in interaction with other substances at temperatures up to $2000 \mathrm{~K}$ and pressures up to a few hundred atmospheres. The particles can be highly erosive; the process streams of crushed solids are subject to plugging; and the liquid products may solidify if they cool down to normal ambient temperatures. The chemical environment is extremely harsh -with both oxidizing and reducing conditions sometimes present in different parts of the same reaction vessel, molten slag being produced, and hydrogen, sulfur, chlorine, sodium, potassium and other harmful species unavoidably present. Access of instrumentation to the process material, protection of sensors, and prevention of plugging are serious problems. In no other processing industry, perhaps, does one encounter such a range of impediments to 
measurement all at the same time.

The foregoing are difficulties associated primarily with design of sensors to penetrate into or survive the hostile regimes of the fossil energy conversion processes. Other difficulties arise with respect to the control elements (valves, pumps, etc.) which are to be regulated in accordance with the measurements. Control elements which can operate reliably and with acceptable service life are lacking; some of the associated materials problems are discussed elsewhere in the Report. The control schemes which will permit reliable physical integration of unit operations with different time constants to produce a specified output have yet to be conceived*.

\section{b. Plan of presentation}

This section of the report is devoted to a somewhat detailed assessment of three especially important instrumentation problems in coal technology: multiphase flow measurement, particulate monitoring, and temperature measurement. For each there is a review of present methods and their difficulties and shortcomings, and a brief discussion of some possible new techniques which might be useful for making the measurements. Following this discussion, there is provided an illustrative technique-oriented section in which possible applications of acoustic methods are suggested and explored. Before addressing the main topics, to indicate the scope of the problems, we present brief comments on a few other instrumentation needs of possible physics interest. We do not provide a complete or exhaustive catalogue of important needs. For this the reader should consult O'Fallon et al, 1981 .

\section{Instrumentation and control needs and problems}

The process parameters requiring measurement and control in coal utilization processes parallel those required in other chemical processes, but the conventional instruments used in the chemical processing

*For example, in combined cycle electric generating plants it may be necessary to follow changing loads while using a feedstock of varying quality to produce an intermediate fuel which is uneconomical to store. industry generally fail in the most hostile areas of the coal processes.

\section{a. Level measurement}

Reliable measurement of fluidized-bed levels at temperatures up to $1400 \mathrm{~K}$ and pressures near $1000 \mathrm{psi}$ is needed, as is also measurement of the level of crushed solids in hoppers. Reliable control of fluidization and bed level could reduce costs by permitting reduction of freeboard above fluidized beds. In many processes, reliable feed control requires measurement of hopper levels.

Fluidized bed depths are now inferred from differential pressure measurements. Plugging of the pressure taps presents a problem which can be alleviated by proper design of a purging system. Measurement by a more direct method would be preferable. Some hopper level instruments based on vibrating elements, capacitance, and acoustic sensing are available.

\section{b. In-situ analysis of solid and/or liquid}

\section{process streams}

Sulfur, ash, heating value, moisture, and volatiles content of dense phase streams must be monitored for process control in coal-using plants. The streams may be coal on a conveyor belt, coal within a pipe, or other solid or liquid streams such as mineral residue or char slurried with water. In many cases the needed information can be inferred from a knowledge of the elemental analysis of the material. Sulfur, moisture, and BTU content are particularly important parameters of feed coals. Carbon/ash ratio in residues is an important indication of process efficiency.

Sampling followed by conventional analysis can, in principle, give the necessary information, but not quickly enough for use in process control. In addition, sampling is difficult to achieve with a high temperature, high pressure system; and when achievable, is made representative only with difficulty. Moreover, the composition of whatever sample is obtained will vary with changes in temperature and pressure in the process and associated sampling apparatus. For process control, an in-situ technique yielding results in minutes or less is needed.

A continuous on-stream automatic sulfur 
meter has been developed (Stewart et al, 1974; Bozorgmanesh et al, 1980). It is based on analysis of prompt gamma rays following capture of (thermalized) neutrons and has successfully monitored the sulfur content of coal feed streams. Multi-element monitors for coal feed streams also have been developed (Gozani et al, 1979; Cekorich, 1979). These are currently undergoing tests in coal preparation and blending plants for a power station. Some work has been done on analysis of gamma rays from inelastic neutron scattering using neutrons from a $14 \mathrm{MeV}$ generator (Herzenberg, 1979). Oxygen, which is not detected with thermal neutron capture, and also carbon respond well to this technique. Large angle elastic scattering of few MeV neutrons also shows promise for identification of important elements in coal streams (Smith, 1981).

In addition, production of short-lived radioisotopes can yield information on elemental concentrations and can permit flow velocity measurement by detection of the moving radioactive tag downstream from a pulsed neutron source. Other work is concentrating on extending all of the above neutron-based techniques to flows in pipes as well as to transport on open conveyor belts or in hoppers (Herzenberg et al, 1979). Moisture determination by microwave, capacitance, and neutron thermalization techniques is being investigated (Brown et al, 1979).

\section{c. Gas analysis}

Measurement of molecular composition of gas and detection of harmful species are needed at temperatures up to $2000 \mathrm{~K}$ or higher and pressures of 1000 psi -- with response times near one second. "Harmful species" include $\mathrm{Na}, \mathrm{K}$, and $\mathrm{Cl}$ which are injurious to equipment, as well as other environmentally hazardous substances.

Gasification plants need rapid molecular composition measurements of the product gas for process control. Pressurized fluidized-bed combustion (PFBC) and other combined cycle systems in which gas turbines are used require monitoring of alkali content of the gas for protection of the turbine, which can be severely damaged in a matter of seconds (Herzenberg et al, 1981).

High temperatures and pressures make direct access to the gas stream difficult. Sampling is too slow in many cases -- and probably unreliable as well -- since constituents may condense or undergo chemical reactions while the gas is being withdrawn and cooled.

Gas chromatographs have been used for molecular analysis, but suffer the same kind of sampling problems. In addition, the measurement generally consumes several minutes or more. A mass spectrograph system has recently become available and has been tested with encouraging results (EPRI, 1979). It, too, is subject to sampling bias, but it has a short response time.

A prototype in-situ infrared gas analysis system has been tested with some success on the product stream of an entrained-bed gasifier (Zweibaum, 1978). Other current work is directed to in-situ laser based detection of gas species, particularly of trace elements (Sullivan and Jensen, 1981). An isothermal gas sampler has been developed and is awaiting tests on a fixed-bed gasifier (Bump, 1979).

\section{d. Pressure}

Conventional pressure measurements involve the introduction of pressure taps into the system. If the taps become clogged with process material, the measurement cannot be made reliably. One way to alleviate this problem is to purge the taps; this, however, may undesirably alter the composition of the process stream. Another alternative is to isolate the process stream from the pressure sensing line by a movable diaphragm; this presents problems if the process material is abrasive or corrosive, in which case it may attack the diaphragm material.

In fact, the great weakness of head-type flowmeters (those which measure flow velocity by sensing the pressure differential across a restriction) is that the required pressure measurements cannot be made satisfactorily.

\section{Multiphase mass flow measurement}

Because coal is a solid, the task of accurately measuring and controlling coal feed into reactors and combustors is greatly complicated. In fact, improved devices for measuring the flow of solids (usually in liquid slurries or gas entrainment) must be regarded as possibly the most critical and widely shared instrumentation need of the advanced coal technologies now under 
development. As has been indicated in Section IV A of this report, gasification, liquefaction, pressurized fluidized bed combustion, and magnetohydrodynamic power generation all require the feeding of crushed or pulverized coal -- usually into a reaction vessel at high pressure. There may also be internal streams containing solids which require monitoring and control.

The temperatures and pressures encountered may range to $2000 \mathrm{~K}$ and to a few hundred atmospheres. The material flows involved may be typically 200 to 2,000 tons per hour for demonstration or commercial plants, with pilot plants smaller by a factor of about 100. Any device inserted into these process streams is exposed not only to the temperature and pressure of the stream, but also to the serious hazard of erosion combined, in many cases, with corrosion. Any device that obstructs the flow, moreover, may carry with it the threat of plugging. For all of these reasons non-invasive methods of measurement are highly desirable. Finally, in some applications extremely short response times are needed -- as short as milliseconds for MHD.

For control purposes, the control quantity of interest is the mass flow rate, $\rho v A$; where $\rho$ is the density, $v$ the flow speed, and $A$ the pipe cross sectional area. Since few sensing techniques respond to mass flow directly, it is generally necessary to combine separate measurements of velocity and density for a calculated mass flow determination. By the same token, with a multi-phase stream, the proportions of individual constituents must somehow be determined. For dry solids at atmospheric pressure, mass flow rate can be satisfactorily measured by a weighing system -- either a weighed conveyor ("gravimetric feeder") in which the rate of loading and belt speed are controlled to deliver solids at the desired rate, or a weighed hopper in which the rate of weight change is monitored. But if a gravimetric feeder is to be used with a pressurized system, there is likely to be substantial and variable feedstock inventory between the point of measurement and the point of injection into the reactor proper. Under these conditions the measurement may be useful only for giving an average over an hour or longer. The weighed hopper is limited in accuracy by the tare weight relative to the weight of solids in the tank. In a pressurized or hot system, moreover, it is difficult to adequately control and compensate for forces exerted on the tank by connecting equipment. In practice these considerations, as well as difficulties due to vibrations, limit the usefulness of the weighed hopper to averages over an hour or more.

Positive displacement meters or pumps provide a means for absolute monitoring of volume flow. They are very accurate for clean liquids, but the accuracy deteriorates rapidly in the presence of abrasive solids, which change the calibration and cause leakage at the seals. A positive displacement pump often can be used to provide a good approximate indication of the flow rate.

Existing devices for fluid flow velocity measurement in the chemical process industry are mostly "head-type" meters -- i.e. they measure the fluid pressure differentials associated with presence in the flow path of some type of obstacle or venturi. Elementary single phase flow theory (Bernoulli's Principle) predicts that the volumetric flow will be proportional to the square root of the pressure differential. In practical systems (multiphase, possibly turbulent flow), it is found that the ratio of volumetric flow to the square root of the pressure differential as a function of flow speed increases to a maximum, then decreases to a relatively constant value for further increase in flow speed. The head-type flowmeters are considered to be usable only for flows in the range where the ratio is relatively constant, i.e. the volumetric flow is proportional to the square root of the pressure differential. Although various geometries have been explored, it has been difficult to extend the usable flow range low enough for typical slurry applications. Mixed phase media containing solids give rise to plugging problems with the pressure measurement taps; and the flow modifying obstacle or venturi is itself subject to very rapid erosion: Clearly some alternative means of measuring slurry flow must be developed.

In the minority of cases where water based slurries are involved, the magnetic flowmeter is a good candidate for measuring the water flow velocity (which will be related to the velocity of the solids). This device sets up a magnetic field transverse to the flow and measures the induced EMF; it has good dynamic range and accuracy, and is non-intrusive. It is relatively expensive and has not yet been developed for high pressure or high temperature application.

An interesting new device is the Coriolis flowmeter. As indicated in Fig. IVB-1 the 
instrument consists of a U-shaped tube or pipe section through which passes the flow to be measured. An (oscillatory) angular velocity is imparted to the U-tube by some suitable means, the axis of rotation lying in the "plane" of the U-tube, orthogonal to its legs, and at some distance from the bend of the $U$. Expressed in the (non-inertial) frame rotating with the U-tube, the fluid flowing in the two legs experiences oppositely directed Coriolis forces which in turn are transmitted to the walls of the tube. Since the tube is not perfectly rigid it exhibits a measurable torsional deflection from which mass flow can be calculated. The device on a small scale has undergone tests on flow of gas entrained solids, and the results were promising (Baucum, 1979). Further tests are indicated for solid-gas and solid-liquid systems. The question of scale-up and erosion during long-term operation has yet to be addressed.

Acoustic flowmeters are beginning to be used for measurement of slurry flow, although commercially available instruments are limited to temperatures below $150 \mathrm{C}$ or so. These instruments, being non-intrusive, are not likely to cause plugging or to have the sensor damaged by the process material.

Passive acoustic instruments simply detect the flow noise and interpret changes in the sound as an indication that something has changed in the system. The simplest systems monitor the total sound emittes while more sophisticated devices may perform spectral

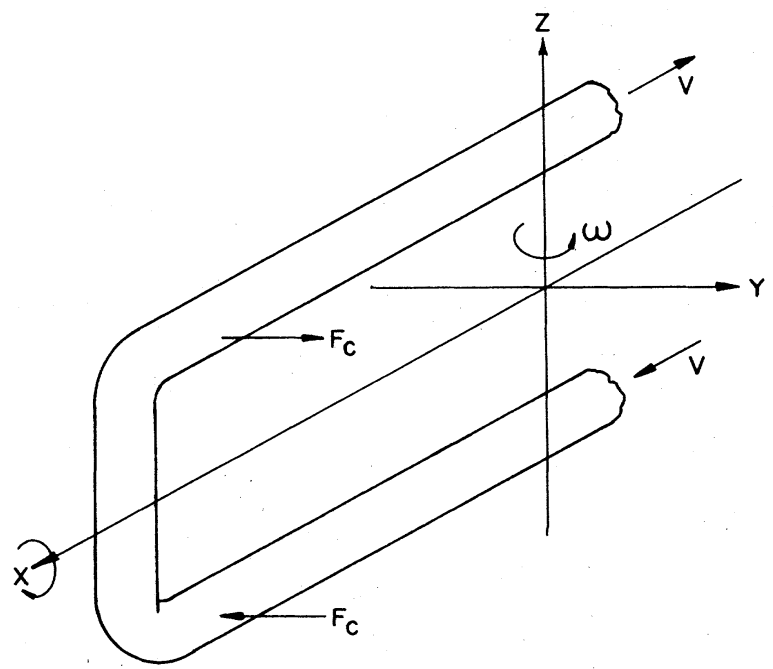

FIG. IVB-1. Coriolis flowmeter. analysis in which different portions of the spectrum are related to various operating conditions. There are active instruments in which a transmitter/receiver pair detects the difference in upstream and downstream transmission time. This can then be related to the flow velocity.

The most common active acoustic (or ultrasonic) flowmeter is the doppler shift instrument in which the frequency shift of waves scattered from the moving particles is related to the particle velocity. Ultrasonic flowmeters are fairly expensive, but their dynamic range is good. Their accuracy is a function of the signal collection time and may be brought within $1 \%$ if a collection time of the order of 10 seconds is acceptable. Slurry applications generally demand lower frequencies than clean liquids - - usually below $500 \mathrm{kHz}$ - - because of increased attenuation ascribable to acoustic scattering by the slurry particles. A high temperature doppler slurry flowmeter is under development and is operating well in pilot plant tests (Karplus and Raptis, 1981). Further development may include the use of high temperature transducers.

Signal processing by cross correlation can be used with a variety of signal types to yield flow velocity information. The covariance of signals from axially separated sensors, regarded as a function of time delay on the upstream signal, has its maximum at a value corresponding to the transit time of the stream from the first sensor to the second, provided that fluctuations in the variable being sensed move with the process stream. This technique has been successfully tested on a toluene and char slurry line at the HYGAS Coal Gasification Pilot Plant using both acoustic sensing and capacitive sensing (Raptis et al, 1978; Managan et al, 1978; Sheen and Raptis, 1979).

Capacitive instruments measure the effective dielectric constant of the process stream through capacitive plates imbedded in a non-conducting liner within the pipe. In the case of a two-phase process stream for which the dielectric constants of the individual phases are known, it is possible to deduce the relative amounts of the two phases present. This provides knowledge of the density of the phase of interest. Segmenting the sensing electrode in the axial direction can provide a simultaneous flow velocity measurement by the cross correlation technique. Thus a single set of sensing elements can obtain data for a 
computed value of mass flow.

With tagging methods, time of transit information can be gained without

the complexity of cross-correlations. Thus one technique under development for monitoring flow velocity uses Pulsed Neutron Activation (PNA)to induce a radioactive tag in the process material. The process stream is irradiated through the pipe with a pulsed 14 MeV neutron generator to activate susceptible elements, such as ${ }^{16} 0$, and passage of the radioactive tag is detected at a downstream station. Initial tests have been carried out using a slurry flow loop with a $14 \mathrm{MeV}$ neutron generator, and the results are promising (Herzenberg, 1979). A system based on this technique may prove to be too costly for routine multiple installations within a plant. However, since it does not require physical modification of the piping, it may be very useful as a movable calibration standard for installed flow instrumentation.

\section{Monitoring of particulates}

\section{a. General discussion}

Particulate matter is involved from the outset in those utilization processes for which the first step is comminution to assure intimate mixing of coal with reactants, solvents, or entraining fluids. Depending upon the process, subsequent reactors or combusters change the chemical and physical properties of particulate matter, remove particles, or produce new particles. The observation of these changes in particulate matter on a single-particle, real-time basis is fundamental to a detailed understanding of the processes. At the end of the process, effluents are delivered to the environment. Monitoring of effluents for particulates is primarily for the protection of the environment although some process control information may be obtained.

A number of methods for the measurement of particle size distribution, some of which are tedious and require care in calibration, are reviewed by Allen (1975). Descriptions of monitors used for ambient air or in-stack measurements are contained in a comprehensive workshop report by Lundgren et al (1979). Techniques for the study of heterogenous combustion are reviewed in a paper by Chitier (1977).
Advances in 1 aser and microprocessor technologies have led to the development of several laser devices, one of which can simultaneously measure particle size and velocity on a single-particle, real time basis. The applicability of modern particulate monitors to a wide variety of problems besides those of coal utilization has stimulated the commercial manufacture of a number of systems (Robinson, 1981).

The major problem which confronts all of the monitor systems to a greater or lesser degree is that of monitoring a process stream of corrosive fluids or gases at high temperature and pressure. Furthermore the density of particulate matter and the physical properties of the non-particulate matter can mitigate against the full utilization of modern single-particle real-time monitors. A series of symposia have been organized on the special instrumentation and control problems which arise in fossil energy processes and the proceedings of these symposia provide excellent summaries of the progress in meeting these problems (Symposia on Instrumentation and Control, 1977, 1978, 1979, 1980, 1981).

Table I presents a selected list of particulate monitors in four groups: prompt single particle counting and sizing devices, high-speed photography, prompt size distribution analyzers, and particle size sorting and collection devices. Acoustical methods are not listed in the Table, but also are beginning to be used (Robinson, 1980).

Individual particles and sizes are measured in real time in the first group of monitors in Table $I$. In the laser visibility method, both the size and velocity are measured. Where conditions permit in situ measurements, information is obtained about the time dependence of the local process stream velocity. High speed photography measures both size and velocity, but the output is delayed. Obviously video recording techniques can reduce the delay.

The prompt size distribution analyzers measure the effects of many diffraction or scattering events and the results are prompt in that the data are analyzed on line with minicomputers. The size range of the photon correlation spectrometer is extraordinary, but the device is vulnerable to error due to spurious light.

The particle size sorting and collection devices use various properties of particles to separate different size particles from aerosol samples. The most commonly used devices in 
TABLE I Particle Monitor Systems

Techniques

Comments

$\underline{\text { References }}$

-PROMPT SINGLE PARTICLE COUNTING AND SIZING DEVICES

\begin{tabular}{|c|c|c|}
\hline $\begin{array}{l}\text { Ratio of laser light } \\
\text { scattering at forward } \\
\text { angles }\end{array}$ & $\sim 0.1 \mu \mathrm{m}$ to $5 \mu \mathrm{m}$ & $\begin{array}{l}\text { W. D. Bachalo, J. } \\
\text { Geffken and G. Weth, } \\
1978\end{array}$ \\
\hline Laser visibility method & $\begin{array}{l}\text { Laser doppler } \\
\text { velocimeter. Size } \\
\text { and velocity measure- } \\
\text { ment } \sim \mu \mathrm{m} \text { to } 200 \mu \mathrm{m}\end{array}$ & $\begin{array}{l}\text { W. M. Farmer, } 1976 \\
\text { W. D. Bachalo, } 1980 \\
\text { N. A. Chigier, } 1976\end{array}$ \\
\hline Image characterization & $\begin{array}{l}\text { Forward scattering of } \\
\text { laser light with mask } \\
\text { array }\end{array}$ & $\begin{array}{l}\text { R. G. Knollenberg } \\
1978,1979\end{array}$ \\
\hline Non-laser Light Scattering & $\begin{array}{l}\sim 0.3 \mu \mathrm{m} \text { to } \sim 10 \mu \mathrm{m} \\
\text { Range varies with } \\
\text { system design }\end{array}$ & $\begin{array}{l}\text { D. D. Cooke and } \\
\text { M. Kerker, } 1975 \\
\text { K. T. Whitby and } \\
\text { K. Willeke, } 1979\end{array}$ \\
\hline HIGH SPEED PHOTOGRAPHY & $\begin{array}{l}\text { Size and velocity } \\
\text { measurement. } \sum_{2} \mu \mathrm{m}\end{array}$ & $\begin{array}{l}\text { N. A. Chigier, } 1976 \\
\text { W. J. McLean } \\
\text { D. R. Hardesty and } \\
\text { J. H. Pohl, } 1980\end{array}$ \\
\hline
\end{tabular}

PROMPT SIZE DISTRIBUTION ANALYZERS

Laser Diffraction $\quad 1 \mu \mathrm{m}$ to $1800 \mu \mathrm{m}$

J. Swithenbank,

J. M. Beer, D. S.

Taylor, D. Abbott

and G. C. McCreath, 1976

Photon Correlation

Statistical Analysis

Spectrometry

of Brownian motion.

$\sim 3 \mathrm{~nm}$ to $3000 \mathrm{~nm}$

H. Z. Cummins and

E. R. Pike, 1974 ,

1977

Vulnerable to spurious

light.

\section{-PARTICLE SIZE SORTING AND COLLECTION DEVICES}

Inertial Classification

Electric Mobility

Diffusion Battery

Filters
Includes impactors, cyclones, centrifuges. Size range depends on

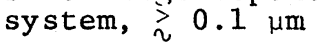

$0.005 \mu \mathrm{m}$ to $\sim 1 \mu \mathrm{m}$

D. A. Lundgren et a1, 1979

B. Y. N. Liu, D. Y. H. Pui and Abde Kapadia, 1979; K. T. Whitby and W. E. Clark, 1966

D. Sinclair, R. J. Countess, B. Y. H. Liu and D. Y. H.

Pui, 1979 dizes dependence of diffusion coefficient on size

T. Allen, 1975;

C. E. Billings

and J. Wilder, 1970 
(Linear Magnitude Scale)
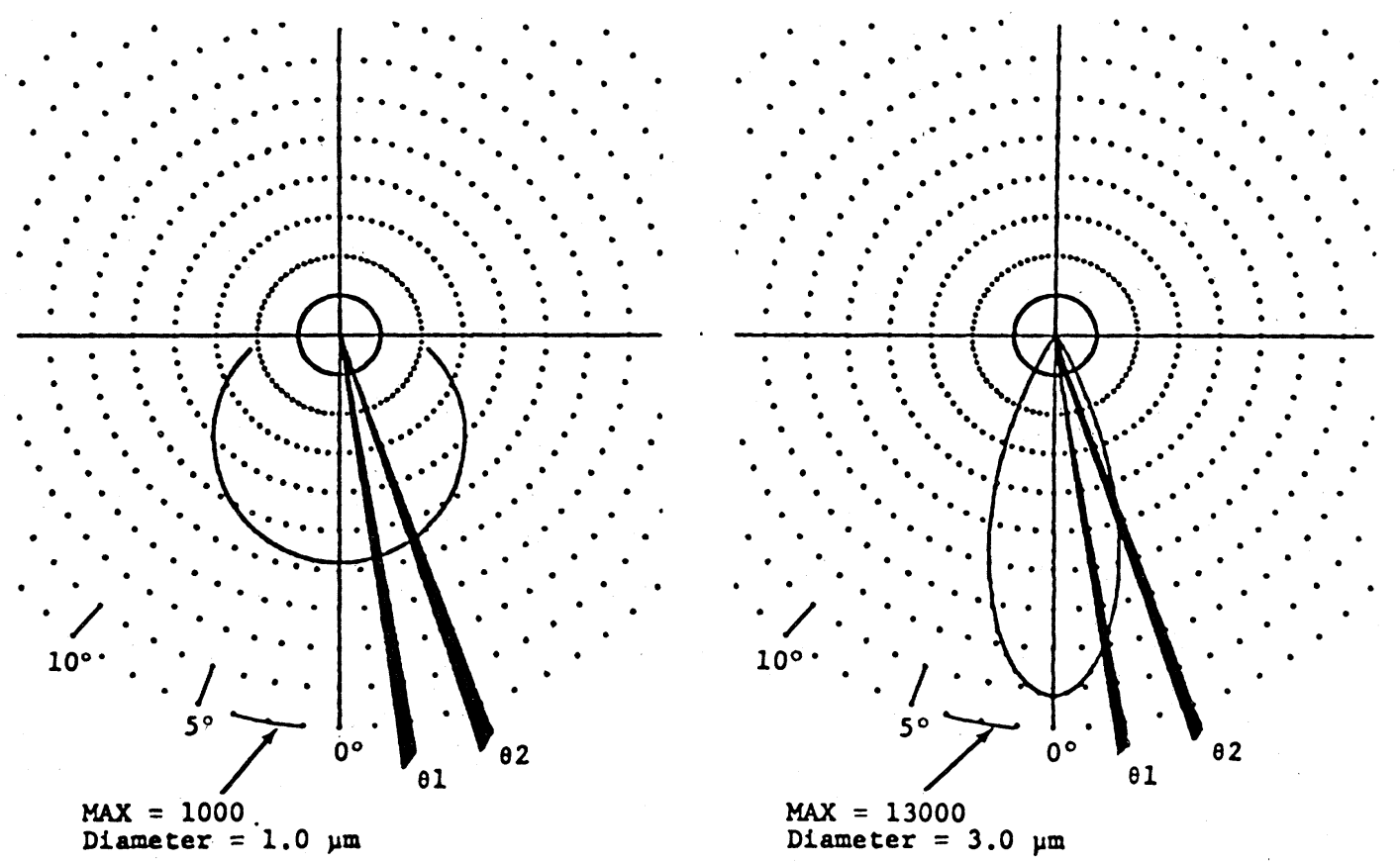

EXPANDED FORWARD SCATTER LOBES SHOWING COLLECTION ANGLES FOR INTENSITY RATIOING

FIG. IVB-2. Dependence of forward scattered light intensity (angular distribution) on particle size.

ambient air or stack gas measurements are the inertial classification monitors.

In the remainder of this subsection, three laser devices and one inertial classifier are discussed and summary remarks are presented in an overview.

\section{b. Intensity ratio of forward scattered laser light}

This device uses the dependence of the scattered light angular distribution to measure the particle size (See Fig. IV B-2). Far forward angles are selected to minimize the uncertainties due to the variations in particle shape and refraction index. The optical system described by Bachalo (1978) is shown in Fig. IV B-3. This method is capable of measuring sizes in the range $0.1 \mu \mathrm{m}$ to $5 \mu \mathrm{m}$ with particle number densities limited to less than $10^{5} / \mathrm{cm}^{3}$ and count rates less than $10^{5} / \mathrm{sec}$. c. Visibility method using split laser beams

Particles in the size range 2 to $200 \mu \mathrm{m}$ can be measured by crossed beam interferometry (Farmer, 1973; Bachalo, 1980). The laser beam

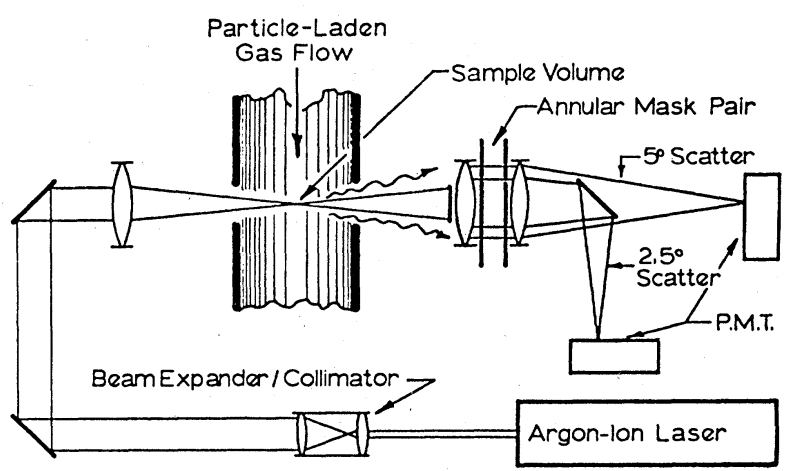

SCHEMATIC DIAGRAM OF THE RATIOING OPTICS SYSTEM

FIG. IVB-3. Schematic diagram of the ratioing optics system. 


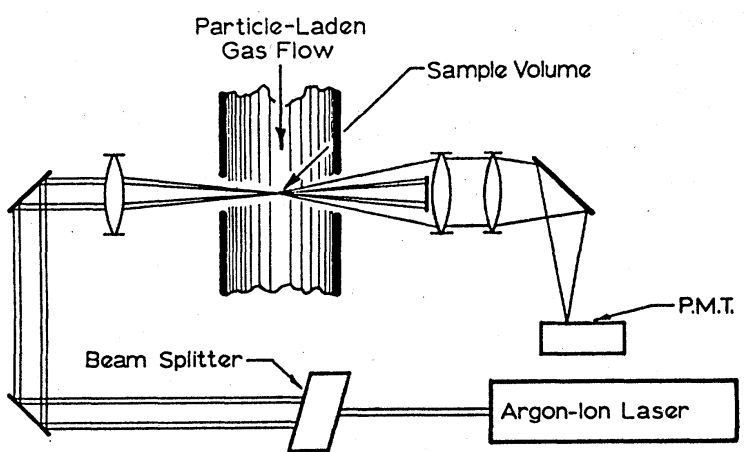

SCHEMATIC DIAGRAM OF THE PARTICLE SIZING INTERFEROMETER OPTICS

FIG. IVB-4. Schematic diagram of the particle sizing interferometer optics.

(See Fig. IV B-4) is split into two components which subsequently intersect to form the measurement volume shown in Fig. IV B-5. Size information is contained in the "visibility" of the signal given by

$$
\mathrm{V}=\frac{I_{\max }-I_{\min }}{I_{\max }+I_{\min }}
$$

where I is the detector photocathode current measured at the maximum and minimum of the modulation shown in Fig. IV $B-6$.
Modulation of the signal results from the passage of the particle through the bright and dark fringes. Variation of the depth of modulation is a function of particle size relative to the fringe spacing. Particles smaller than the fringe spacing will scatter light to trace out the sinusoidal fringe intensity distribution. Particles larger than the fringe spacing will affect the light scattered into two or more fringes depending upon the size of the particle. The visibility as such is the ratio of the scattered intensity for the particle centered on the brighest fringe to that for centering on the adjacent fringe. The relationship between visibility and particle size is mathematically complicated, but with the aid of computers it is promptly determined.

The system determines the particle velocity by operating as a laser velocimeter. Consequently this device measures the number density, particle size, velocity and, from the velocity, information about variation of velocity or turbulence. By acoustically driving the particle, the aerodynamic size can be measured (Mazumder et al, 1981). The aerodynamic size is the size of the particle of unit density which would have the same settling velocity as the particle in question. The environmental dispersion and deposition of particles from an aerosol are usually discussed in terms of aerodynamic diameter.

\section{ENLARGED CROSS-SECTIONAL VIEWS OF THE BEAM CROSSOVER REGION}

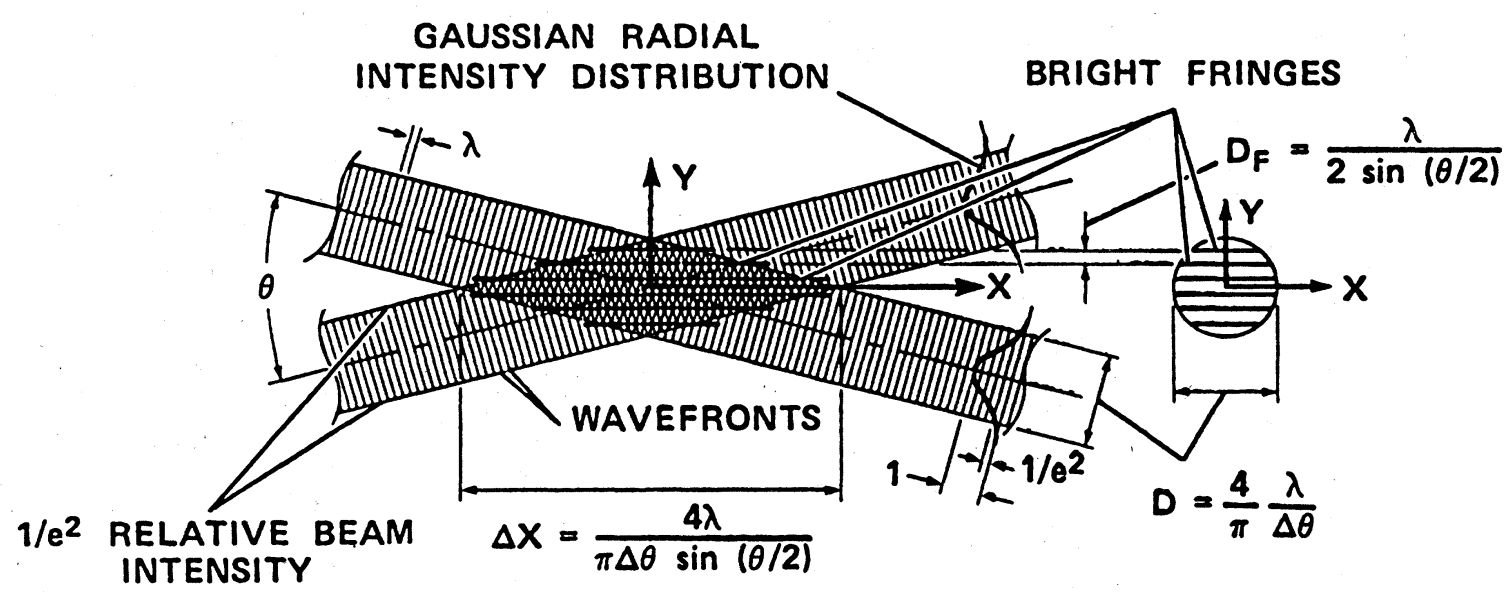

FIG. IVB-5. Enlarged cross-sectional views of the beam crossover region. 


\section{d. Particle sizing using laser diffraction}

When illuminated by parallel monochromatic light, spherical particles (or droplets) produce a diffraction pattern the intensity of which is given by well known Bessel functions. The pattern consists of a series of bright and dark rings superimposed on the smaller geometric image. By placing a

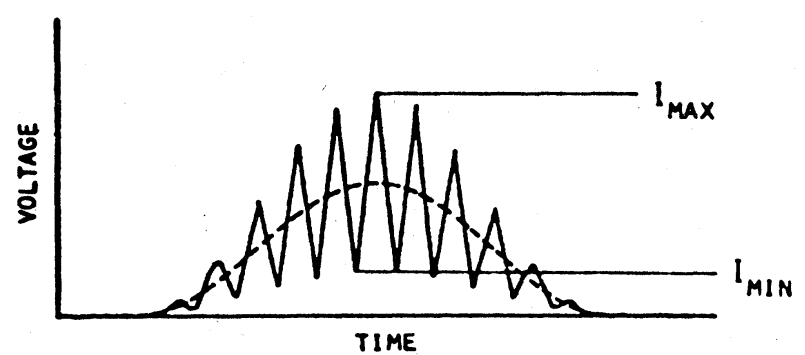

(A) DOPPLER BURST SIGNAL

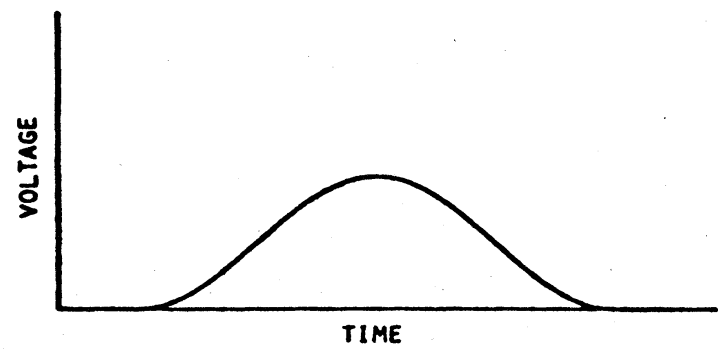

(B) PEDEstal COMPONENT

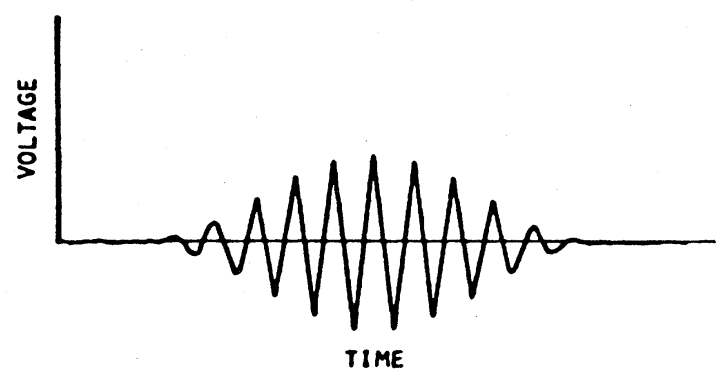

(c) DOPPLER COMPONENT

Doppler burst signal showing the Doppler and pedestal components.

FIG. IVB-6. Doppler burst signal showing the Doppler and pedestal components. lens between the particles and a detector as shown in Fig. IV B-7, the undiffracted light is focused to a small spot that is the center of the detector with a much larger diffraction pattern surrounding. Movement of the particles does not cause a shift of the pattern. Particle diameter is determined since the pattern diameter is inversely proportional to the particle diameter. In a sense, the lens functions as a Fourier transform lens since the diffraction pattern is a Fourier transform of the field distribution over the particle.

Fraunhofer diffraction, or far-field approximation, works well for particle sizes greater than the wavelength of the light. Using a HeNe laser $(0.6328 \mu \mathrm{m})$, particles can be measured with sizes down to $1 \mu \mathrm{m}$. Shorter wavelength light and complex Mie theory must be used for smaller size particles.

Signal processing from various parts of the receiver requires microcomputer capability. The use of this technique for droplet and particle size distribution measurements is discussed by Swithenbank et al (1976) and recent applications are reviewed by Weiner (1979). This method does not count single particles but rather yields a measurement of size distribution for a number of particles in the sample.

\section{e. Photon correlation spectrometry}

Although how diffraction and Brownian motion are to be taken into account in particle sizing was understood long ago, devices for prompt sizing awaited the development of the laser and modern microcomputer electronics (Cummins and Pike, 1977).

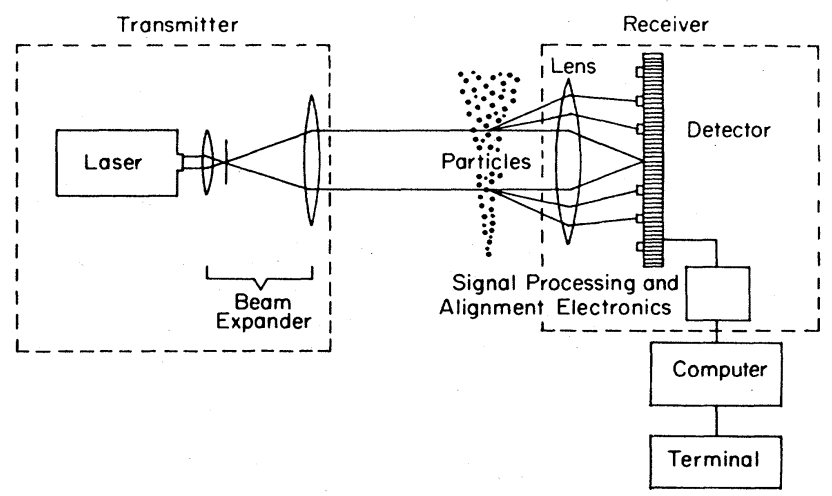

FIG. IVB-7. Particle sizing using laser diffraction. 
The photon correlation technique uses the microcomputer to establish the auto-correlation functions for light scattered from particles which move in and out of the detection volume due to thermal motion. The rate and way the particles move is a direct function of their size, the larger particles moving more slowly than the smaller ones. A measure of this particle movement determines the size and other information related to the particle. In particular, the self correlation in time determines the diffusion coefficient. The diffusion coefficient in turn determines the size of the particle through the Stokes-Einstein equation in which the size is inversely related to the diffusion coefficient. The photon correlation spectrometer is not an individual particle counter, but rather obtains statistical information from a large number of particles (Cummins and Pike, 1974).

\section{f. Impactors}

Several devices utilize the inertial properties of particles (Lundgren et al, 1979). The most commonly used device for in-stack monitoring of combustion products is the impactor. The schematic of a cascade impactor is shown in Fig. IV B-8. By passing the aerosol stream through successively smaller jet apertures with corresponding higher velocities, particles of successively smaller size and mass are impacted on plates which are subsequently removed and the impacted material weighed. This device does not offer a prompt measurement, much less an individual particle measurement, but it does provide a simple and reliable means for time-integrated size distribution measurement which is an accepted standard for compliance with regulations. Units can be mounted in a duct, however, sampling trains are used in most applications.

\section{g. Overview}

Historically the measurement of particle size distributions has been a time consuming, tedious process requiring repeated calibration. A large number of techniques is available (Allen, 1975) and only a few have been discussed in the foregoing material. Optical microscopy offers the most direct means of observing particles but it requires trapping of the particles and has little or no utility in the observation of particles in process streams.

The general problem of measuring the size, velocity, and temperature of a particle in the frequently hostile environment of coal combustion or coal utilization processes has not been solved. Sampling trains (USEPA, 1977; USEPA, 1976; Chitier, 1979) are often required either to protect the equipment or to provide controlled dilution or both. Nevertheless substantial progress has been made, most recently due to the combined application of laser and microcomputer technologies. Certain of the long established technologies such as filtration are the subjects of recently renewed interest. In particular, electrification effects in fabric filtration are being actively investigated (Penny, 1977; Lamb and Constanza, 1980).

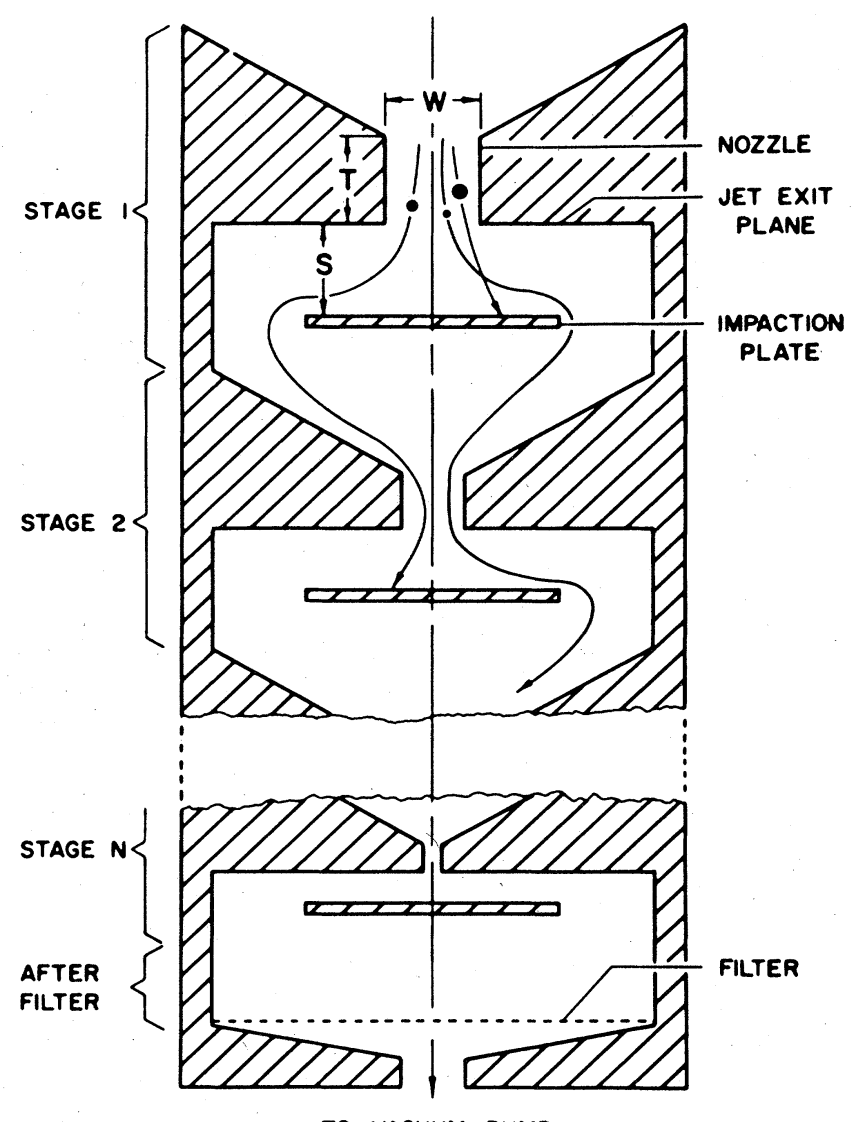

TO VACUUM PUMP

Schematic of cascade impactor with trajectories shown for particles of three different sizes.

FIG. IVB-8. Schematic of cascade impactor with trajectories shown for particles of three different sizes. 


\section{Temperature measurements}

Temperatures of gases and mixed phase media must be measured under both reducing and oxidizing conditions up to about $2500 \mathrm{C}$ and under pressures up to 1000 psi, sometimes within a few seconds. Temperature monitoring is critical for safety and process control in all advanced fossil energy processes, since temperature is probably the single most important indicator of the operating state of the reactor or combustor and can change by 100 $C$ per second in some processes.

Temperature measurements are needed not only for process control in full scale plants but also for scientific and engineering diagnostic purposes in less than full scale plants, e.g., pilot plants or even smaller laboratory models. For diagnostic purposes, on-line real-time temperature measurements, though always desirable, may not be necessary; data can be stored and analyzed later. For process control and safety, on the other hand, on-line real-time measurement ("monitoring") generally is essential; it is often the case that failure to hold a process temperature within specified bounds can result in major structural damage or even a life-endangering explosion. Process yields, moreover, often depend very sensitively on temperature.

Pilot or demonstration plants, though less than full scale, also are not without significant hazards and must be provided with on-line monitoring adequate for effective control. Temperatures measured for diagnostic purposes sometimes must be reconstructed as nearly instantaneous functions of time, even though the result need not be known to the experimenter in real time. In an entrained-bed gasifier, for example, the residence time of the gas in the reactor is only a few seconds. To study the complicated reaction kinetics in such a rector, temperature time resolution of a fraction of a second is highly desirable.

In general one expects that many more parameters must be measured for diagnostic and developmental purposes than are necessary for process control. For process control (e.g., in a coal liquefier) it may be sufficient to monitor the temperature at less than a dozen selected points, but for diagnosing the operation of an experimental liquefier, temperature measurements at hundreds of points may be desirable.

The desire for temperature measurements for diagnostic purposes may additionally increase because conditions at a single point in a reactor may be characterized by several different "temperatures." Unless the system is in local thermodynamic equilibrium, the kinetic and radiation temperatures at any point need not be the same, and each of these may differ from the molecular vibrational excitation temperature, or that describing the atomic level populations. Though it may be reasonable to assume local thermodynamic equilibrium in the interior of a high presure liquefier, this assumption is at least questionable in the interior of a gasifier. The gasifier diagnostician therefore may wish to use simultaneously several instruments, each of which measures a different "temperature."

\section{a. Thermometry for coal technology}

The methods which have been used to measure temperature in the coal utilization industry have been surveyed recently (Argonne, 1980). These methods include thermocouples; optical pyrometry; electrical resistance thermometers, including thermistors and devices which infer the resistance from the measured Johnson noise; acoustic devices; crystal thermometers, which measure changes in the crystal resonant frequency; "filled thermal elements," (e.g., conventional mercury-in-glass thermometers); bi-metallic thermometers; and even color indicators (paints which change color as a function of temperature). This list by no means exhausts the methods which have been used or suggested. Probably the most complete surveys of temperature measuring methods are to be found in the Reinhold Volumes edited by Herzfeld (1962) and Plumb (1972). A very recent study (Herzenberg, 1981) discusses still other modern temperature measuring methods, and offers a very readable and worthwhile analysis - relevant to the discussion below -- of the problem of measuring temperature in fluidized-bed combustion reactors

It is impractical to discuss all methods here; the following discussion concentrates, for illustrative purposes, on two methods -thermocouples and optical techniques. These two, and perhaps electrical resistance thermometers and acoustic devices, are the methods we believe most likely to prove useful at the high temperatures and in the hostile environments encountered in coal technology. Potentialities of some other methods listed in 
the references which have been cited may not have been sufficiently examined, however.

Thermocouples: Publications on thermocouples and thermoelectric materials are very numerous; we make no attempt here to summarize or reference this literature. (See Plumb, 1972.)

In practice, the Type $K$ thermocouple (chromel-alumel) has been used more widely than any other type, but various authorities report advantages for other newly-developed thermocouple types (e.g., nicrosil-nisil); these are useable to about 1250C. Above $1250 \mathrm{C}$, to about $1700 \mathrm{C}$, use of platinum-rhodium (Type B) thermocouples has been recommended, and tungsten-rhenium alloy thermocouples are said to be useful as high as 2760C. These latter type thermocouples are quite expensive, however, particularly in view of the large dimensions of full scale coal utilization reactors, whose central temperatures often are required. It is not unusual to employ -- and destroy -- thousands of feet of thermocouple wire in a single diagnostic test series. Alternative less expensive thermocouple alloys are much needed, especially for measurements at the higher temperatures. Desirable characteristics obviously include the capability of being drawn into long wires and bent without breaking, together with stability of calibration over long periods of handling and exposure to high temperatures.

Probably the most stringent demand placed upon thermocouples by coal technology is the ability to withstand the highly corrosive environments prevalent in coal reactors. To date, no thermocouple type is known that can withstand this corrosion long enough even for diagnostic tests. Even with thermocouples enclosed in corrosion resistant sheaths or "thermowells", this difficulty persists. For example, in one experimental gasifier (Pitcher, 1978) thermocouples in silicon carbide thermowells have rarely survived more than a few days in the ambient mixture of steam, methane, oxygen and coal particles at $1300 \mathrm{C}-1600 \mathrm{C}$. Experience with sheathed thermocouples in magnetohydrodynamic (MHD) plasmas has been similar.

Thermowells, even if completely corrosion resistant, introduce characteristic problems of their own. Response times of thermocouples in thermowells are rarely shorter than several minutes. This is far too slow in many situations. Even in steady-state conditions, moreover, use of a thermowell can introduce artifacts and measurement errors associated with heat conduction and shunt electrical conduction in the thermowell-thermocouple combination. In principle, individual calibration of the device can help compensate for such errors, but reliable calibrations applicable to the actual working conditions encountered in a coal reactor or furnace are difficult to achieve. It follows that research seeking to identify improved thermocouple sheathing materials, capable of minimizing the aforementioned errors inherent in thermowell use, while simultaneously resisting the corrosive effects of the coal utilization industry's hazardous environments, should be strongly encouraged. Even more to be encouraged are searches for thermocouple materials themselves capable of resisting corrosion, and therefore capable of being used without thermowells. Although at this time such searches cannot be said to have more than a small probability of success, it should be noted that high temperature thermocouple elemental materials need not be confined to metals or metallic alloys, which do not have a monopoly on conduction by electron carriers (such conduction probably is a prerequisite to finding a measurable thermoelectric emf). Indeed, the seebeck effect has been demonstrated in semiconductors (Frederikse, 1963), and carbon-graphite high temperature thermocouples were being investigated at the turn of the century (Kinzie, 1973). However, irrespective of their obvious unsuitability in coal-burning circumstances, these carbon-graphite thermocouples, with their obvious ductility and fragility problems, illustrate the advantages of metallic thermocouple elements and the difficulties of replacing them.

Optical Techniques: According to Argonne $(1980)$, temperature measurement by optical techniques in the coal utilization industry has not extended beyond employment of radiation pyrometers. The instruments used have mainly been infrared pyrometers operating in the wavelength band from about 1 to 10 micrometers. Assuming the source is a black body, temperature can be inferred from the integrated (over wavelength) radiation intensity in the observed spectral band, or from the slope (as a function of wavelength) of the radiation intensity in the observed band. The task of measuring the spectral slope typically has been accomplished by means of a so-called two-color radiometer, which measures the ratio of the received intensities in two separate narrow bands. 
Depending upon emissivity and equilibration, two-color sampling techniques can occasionally give misleading results. If it is reasonable to assume local thermodynamic equilibrium in coal utilization reactors which operate at high pressures and/or whose working substances have long residence times, then it is correspondingly reasonable to suppose that the radiation is black body in character -especially since these reactor interiors frequently contain many coal particles which strongly scatter and absorb radiation. On the other hand, the possibility that the radiation observed from any given reactor may not be black body cannot be arbitrarily ruled out. Wherever possible, therefore, spectra should be scanned over a sufficient range to permit slope estimates from more than a single pair of frequencies; and/or the radiation temperature should be compared with the indications from a thermocouple placed in the spatial region whose temperature (supposedly) is being sampled by the observed radiation.

It seems unwise to limit consideration just to optical pyrometers. Line widths and line profiles also carry temperature information which for many years has been used in astrophysics, plasma physics, flame chemistry, and numerous other research areas. The coal utilization industry has made little practical use of these techniques. Argonne (1980) mentions spectroscopic thermometers, but says of these that they involve "laboratory techniques seldom employed industrially". This may amount to overlooking a good bet.

For diagnostic purposes immediate temperature readout often is not essential, so that the fairly complicated data processing which may be necessary to infer (e.g., vibrational and rotational) temperatures from molecular spectra should not impede use for pilot plant analysis. With modern computing capabilities, moreover, such measurements may be practical even for process control in full scale plants.

In principle, the radiation sensor for optical temperature measuring techniques can be located external to the reactor being measured, thereby avoiding the corrosive environments. In practice this advantage is muted by the fact that many coal utilization industry reactors -- especially those which are large, operate at high pressure, or contain many carbon particles -- will be optically thick at visible and infrared wave lengths. Under such circumstances, optical measurements of interior temperatures require the insertion of optical probes in protective sheaths with all the attendant corrosion problems discussed above for thermowells.

Even when the interior is optically thin, external sensing of the reactor radiations requires the construction of optical windows in the reactor walls. These may prove vulnerable to slagging or coating. Success in keeping the window clean by "purging" (i.e. by introducing a gas stream to blow away any particles before they can adhere to the surface) has been reported (Ballard, 1981). of course, purging can also introduce experimental artifacts.

Subject to the same problems with windows, reactor opacity, etc., one may probe conditions within a reactor by means of radiation introduced from the outside. (The study of flames by absorption spectroscopy provides a simple and well known example.) Zweibaum et al (1978) have reported what is in effect a microwave absorption spectrum for the BI-GAS pilot plant reactor, in the range 1.6 to 5.5. micrometers. This spectrum shows considerable structure, presumably associated with various molecular species within the reactor; the authors suggest that this structure could be continuously monitored for process control.

Availability of lasers continuously tunable over a broad range of wavelengths in the visible and infrared should make the study of reactor interiors by means of externally introduced radiation much more attractive. Indeed, the measurement of laser Raman and Rayleigh scattering as a means of probing reactor interiors was strongly advocated six years ago in a study of the role of physics in combustion (Hartley et al, 1975). It was pointed out that Raman scattering can be used to measure rotational and vibrational temperatures of the various species in the reactor, as well as the relative densities of those species, and that it even may be possible to determine their spatial distributions. Hartley et al (1975) also discuss other possible laser diagnostic techniques, (e.g., laser-induced fluorescence) and suggest a number of specific combustion research experiments (mainly applicable to flame research however).

Although the potential use of lasers in coal conversion diagnostics and process monitoring has received some attention, we feel that a more intense and systematic effort is needed and worthwhile. Lasers have proved 
to be so versatile a diagnostic tool that we believe they will open up new avenues for coal conversion reactor diagnostics and process control.

\section{b. Temperature measurements and modeling}

As has been explained, under some circumstances reactor interiors are expected to be an optically thick distance from the reactor walls, whereas in other circumstances the reactor interiors may be at optically thin distances from all walls. In the latter case it is possible for measured radiation temperatures at a point to be determined by the temperatures of "hot spots" remote from that point. Similarly, under some circumstances the interior of the reactor can be expected to be everywhere in local thermodynamic equilibrium, whereas in other circumstances there are likely to be differences between the kinetic, radiation, rotational, vibrational, etc., temperatures at a given point in the reactor's interior.

Through reactor modeling, whose potentials and problems are discussed in section IV-C of the report, it may be possible to estimate in advance the interior conditions of the reactor (optically thick or thin, etc.) in order that the appropriate diagnostic sensors and process monitoring controls be incorporated into the design. Reactor models will play an important role in interpreting the temperature and other measurements (e.g., species composition) obtained in cases where local thermodynamic equilibrium cannot be assumed. For reasons such as these, Hartley et al (1975) strongly recommended increased combustion modeling efforts, especially for advanced coal industry reactors. We strongly endorse this recommendation, because of the need for modeling to properly measure and interpret reactor temperatures as well as for other reasons. Further, we agree with Hartley et al that the connection between modeling and diagnostics is a two way street. Good diagnostics, which include temperature measurements, are needed to test and improve reactor models.

Reactor models also have an important role to play in what may be termed indirect measurements of reactor temperatures. Because reactor temperatures often are so difficult to obtain, there is considerable merit to the idea of seeking ways of inferring the temperature from more readily accessible data than those which are needed for "direct" temperature measurements of the sort this report has examined. Of course, all temperature measurements are indirect in a sense, but by "indirect" here is meant inferring the temperature from a relationship which has been deduced via reactor modeling and is valid only in the particular reactor under consideration -- as distinct from some general theoretical relationship (e.g., black-body radiation intensity or Doppler broadening) which is valid for all reactors.

The idea of indirectly measuring the temperature may be particularly important in process control monitoring of hazardous temperature excursions; this possibility is predicated on the existence of a reactor property which changes rapidly with reactor temperature (perhaps even changing significantly before the temperature excursion is well underway) and which is easily measurable with rapidly responding equipment. For instance, to give a purely hypothetical example, modeling might indicate that the concentrations of ethane and other higher (than methane) members of the alkane series measured at the output stream of a gasifier are very sensitive functions of the gasifier reactor temperature, with these concentrations decreasing markedly as the reactor temperature increases. In this event, the gasifier reactor temperature could be conveniently monitored by measuring the concentrations of higher alkanes in the gasifier output stream. This stream is readily accessible, and there exist numerous well-established techniques (mass spectrographic analysis, infrared absorption spectroscopy, laser Raman spectroscopy, etc.) which offer good hopes for measuring these concentrations on line. It is noteworthy that in this sort of indirect temperature monitoring for process control, the modeling need not be so accurate or thorough as to enable reliable determination of the interior reactor temperature from the externally measured higher alkane concentrations; all that is necessary for process control of the reactor temperature is the much easier modeling task of reliably establishing a high correlation between rises in the interior temperature and changes in the externally measured quantities, in this hypothetical case the concentrations of the process stream alkanes. It is strongly recommended that reactor modeling objectives 
include searches for suitable indirect measures of reactor temperatures as well as other important process variables.

\section{Potentialities of acoustics}

The past two decades have seen remarkable improvements in acoustic technology, associated with an equally remarkable growth in the applications of acoustics to studies of all forms of matter, including e.g., plasmas and biological materials. Application of acoustics to instrumentation and control needs of the coal utilization industry has shared in this growth. It may be, nonetheless, that the full potential of acoustic methods in coal technology has yet to be realized.

The remainder of this section speculates on some potentially interesting lines of investigation. We offer some reasons for believing acoustic techniques may, in the future, be still more useful in coal technology, and then put forth some illustrative possibilities (which we think warrant further investigation) for new applications in the field. Because known acoustic applications to the study of matter, like the methods for measuring temperature examined in subsection 5 above, already are so numerous and diverse, the discussion which follows can only touch upon a very. few of the potentialities of acoustics in the coal utilization industry. No attempt is made here to thoroughly survey the literature. For this the reader is directed to Sessler (1968), Carlin (1964), and other articles in the same "Physical Acoustics" series.

\section{a. Reasons for investigating acoustic techniques}

Acoustic and optical techniques share the basic property of being "non-invasive" or "non-perturbative" under ordinary circumstances. Acoustic measurements may be either passive -- listening to the sounds generated in the system being monitored -- or active, sending acoustic waves into a system and monitoring the backscattered or transmitted acoustic signal. Acoustic waves, of course, can be produced or tuned over a very broad band of frequencies and wavelengths.
On the other hand, by increasing the incident power, acoustic as well as electromagnetic waves can be used to heat samples and to cause other chemical and physical changes. That is, they can deliberately be made invasive or even destructive. Changes intentionally induced by acoustic waves conceivably could be industrially useful in e.g., coal liquefaction, or could become the basis for other (not available at lower power inputs) acoustic diagnostic techniques. One such destructive acoustic process, having no obvious laser analogue, is "cavitation", the production of vapor-filled cavities in liquids by acoustic waves whose peak tensions (peak negative pressures, taking into account the external positive hydrostatic pressure) exceed the liquid tensile strengths. In practice liquids can rupture at tensions far less than the theoretical tensile strength, presumably because of the presence of small bubbles capable of growing to large cavities when the tension exceeds $2 \alpha / r$, where $\alpha$ is the surface tension and $r$ is the bubble radius before the tension is experienced.

In sum, acoustic probing of coal utilization reactors has many of the desirable features associated with optical probing, while in other respects being complementary. Because acoustic propagation in a medium depends primarily on its visco-elastic properties rather than on its electromagnetic parameters, acoustic waves can penetrate media which are optically opaque (e.g. metals). For this reason alone, acoustic probing techniques invite attention. One may expect to encounter situations where sound generated within the reactor could be monitored from the outside without any need to insert a detector into the reactor or to construct a "window" through the reactor wall. The problems stemming from fouling of the window, described earlier in the discussion of optical techniques, should be much less troublesome for acoustic waves than for infrared or visible light waves. Another possible advantage stems from the fact that, for distances of the order of reactor dimensions, the propagation time over any chosen path, from which pulse velocity can be inferred, is much easier to measure for acoustic pulses than for electromagnetic pulses. Acoustic pulse velocities should be temperature dependent; their use to monitor temperatures in reactor interiors presently is being seriously investigated, as is explained below. 


\section{b. Present applications of acoustics in}

\section{coal utilization}

Acoustic techniques already are being used, or at least diligently investigated, in the coal utilization industry. As already described, several different applications of acoustics to the measurement and monitoring of multiphase flow (e.g. of coal slurries), involving both passive listening to acoustic noise and active detection of back-scattered externally generated sound waves, have been vigorously pursued.

Acoustic Time Domain Thermometry: Acoustic time domain thermometry, also called acoustic time domain reflectometry or acoustic TDR, has been accomplished in nuclear reactors by inserting into the reactor a wire with one or several designed acoustic impedance mismatches. The times taken for an externally generated acoustic pulse to run down the wire and be reflected back from the mismatch points are then measured. Because the velocity of sound in the wire depends on the temperature, these time measurements permit inferring the wire temperature. Obviously one can infer only the average temperature along a segment of the wire between two impedance mismatches generating reflected pulses; but by making these segments sufficiently short, the average is taken over correspondingly smaller regions. As many as ten wire segments reportedly have been employed in the case of nuclear reactors (O'Fallon et al, 1976).

The main environmental hazard (aside from high temperature) in nuclear reactors is posed by neutrons, which can and actually do transmute the wire carrying the acoustic signal. The environmental hazards (again aside from high temperature) in coal utilization reactors surely include corrosion, as been discussed earlier (under Thermocouples, subsection 5 ), and alteration by solid state diffusion. To avoid these hazards it may be necessary to employ protective sheaths around the wire carrying the acoustic signal. In this event problems akin to those arising with thermocouple sheaths may be encountered. The main such problem with acoustic TDR would be the increased response time associated with the finite thermal conductivity of the sheath. This might nullify one of the principal possible advantages of acoustic TDR, the hoped for rapid response times. On the other hand, problems such as shunt electrical conduction in the sheath material would seem to be less troublesome for acoustic TDR. Because acoustic TDR does not require an electrically conducting element, it is possible that a corrosion-resistant refractory material not requiring a sheath could be found for use in acoustic TDR. The question of alteration by solid state diffusion would remain to be explored separately.

It is conceivable (Papadakis, 1976) that measured attenuations of the reflected signals and observed propagation times could be combined to improve the temperature information attainable by acoustic TDR. The attenuation of sound waves, like the sound velocity, is usually temperature dependent. This idea seems to have been explored neither for coal reactors nor (apparently) for nuclear reactors. Highly temperature-dependent attenuation in the temperature range anticipated may be achievable by taking advantage of the fact that the acoustic attenuation in refractory metal wires has been observed to increase sharply at a temperature equal to about half the melting point temperature (Papadakis, 1976).

Ultrasonic Fluidized Bed Level Measurement: Use of acoustics to measure fluidized bed level height has been proposed (Argonne, 1980; O'Fallon, 1976). This presumes an unambiguous reflection at the upper "surface" of the bed, and one can easily anticipate the sources of error in this technique. Echoes from points above the actual bed level, for example, could result from high concentrations of particulates in the gas above the bed, from strong turbulence in the gaseous atmosphere, from violent foaming at the interface surface, etc.

Despite such difficulties similar approaches might be considered whenever the location of a surface of discontinuity is desired. Proper monitoring of the BI-GAS pilot plant gasifier, for example, requires monitoring of the char level in a char hopper. The possible utility of ultrasonic level measurements in this and other coal technology processes has been examined (Argonne, 1980).

Acoustic Detection of Valve Leaks: An already widely employed method for detecting the development of cracks and other defects in materials is the "acoustic emission" technique, also known as AE (Spanner, 1979). In this method, one seeks to detect the characteristic transient sounds emitted when materials undergo microscopic deformations or 
fractures; an example might be sliding of a grain boundary. Increases in $A E$ precede the development of macroscopic flaws. Techniques for locating the evolving flaw, using appropriate processing of the signals received from several differently situated acoustic detectors, have been developed. Increased use of $A E$ in the coal utilization industry has been advocated in a conference whose objectives resembled those of the present report (Shewmon, 1978).

Control valves must operate against high pressure differentials, at high temperatures, and must handle highly corrosive and abrasive materials. Consequently, on any one day in an operating fossil fuel plant, many valves having important functions are likely to have deteriorated and to need replacement; failure to promptly replace such deteriorated valves sometimes carries a risk of accident capable of causing serious damage to the plant.

Identifying faulty valves by acoustic emission presently is receiving serious study at several laboratories. Although few tests have gone beyond the laboratory stage, there is reason to believe some version of the $A E$ method will be able to detect valve leaks, as well as serious deterioration in valves before leakage develops (Ellingson, 1980).

As a far more general principle, deviations from normal operating conditions in many kinds of equipment are likely to be accompanied by characteristic changes in sound emission, especially when the operation involves fluid flow. While the relatively crude procedure of simply monitoring the total sound emission to detect deviations from normal operating conditions presently is being used in some plants, spectral analysis and other signal processing techniques can yield more detailed information about the operating conditions. Valve leak studies suggest that the valve leak acoustic signal "is basically a broad band random noise signal" (Smith, 1979). Although the specific properties of this random signal (e.g., spectral slope, correlation functions, etc.) apparently have not been investigated, some elementary two-band spectral comparison of the received noise has been found to improve detection of the desired valve leak signal (Smith, 1979).

\section{c. Some possible applications of acoustics to coal technology}

The following topics have not been "researched" in any legitimate sense of the term; they are offered here in the hope that some readers may be stimulated to investigations both broader and deeper than ours.

Use of High Intensity Sound Waves: One possible use of high intensity perturbing sound waves has been recognized in the industry, namely the control of particle emissions in smoke and other fumes (Carlin, 1964). The smaller particles in these emissions, those with sizes less than a few micrometers, are the most difficult particles to remove from the gases carrying them. Agglomeration of the smaller particulates by means of intense acoustic waves, a procedure which has some theoretical basis, is receiving serious examination (Volk, 1976).

Coal Liquefaction: A more direct exploitation of high intensity sound waves -one apparently not yet explored in the coal utilization industry and offered here as a speculation -- is the promotion of direct coal liquefaction (in the presence of hydrogen donors and/or catalysts). It is known that ultrasonic waves can affect the rates of chemical reactions (Basedow, 1979). Therefore, it seems worth investigating whether intense ultrasound beams could improve or help to control the process of coal liquefaction. Of course, it is essential that the energy expended in producing and transmitting the ultrasonic beam not be a significant fraction of the usable energy in the coal liquids involved. (However, because coal liquefaction processes typically require that the coal be heated, the ultrasonic energy dissipated in the coal will aid the heating and will not be entirely wasted.)

If the ultrasonic waves traversing the liquefying coal are intense enough to cause cavitation (recall the earlier discussion in subsection 6a) very complex chemical and physical effects may occur e.g., luminescence (Flynn, 1964). If liquefaction starts at the outer surfaces of coal particles, then exciting cavitation -- which typically erodes the solids at solid-fluid interfaces -- might expose new surface and thereby increase the rate of liquefaction. On this basis, attempts to drive the liquefying coal to cavitation via ultrasonic radiation might merit preliminary consideration. Unfortunately, production of cavitation in the highly viscid media of coal liquefaction is likely to be very difficult, i.e., to require very (perhaps impractically) high acoustic intensities. Any such difficulty will be compounded by the fact that 
cavitation, once it occurs, greatly impedes further transmission of intense acoustic waves into the cavitating medium, because of scattering by the macroscopic bubbles and the acoustic impedance mismatch caused by the bubbles.

Coal Comminution: In many coal utilization processes, efficient operation requires that the coal be fed to the combustor or reactor in the form of comparatively small particles (approximately 100 micrometers in size). Customarily the large coal fragments obtained from the mining process are ground down to these small sizes. Grinding has proved to be expensive however, in money, time, and energy. It has been estimated that $1 \%$ or more of the usable energy of the coal can be expended in the grinding operation (See IV E.). There have been many attempts to achieve size reduction without grinding, primarily chemical fragmentation of coal. Chemical comminution of coal can be achieved, but the chemical processes needed are inconvenient and expensive. (Chemical comminution is discussed in Sec. IV E.)

The possibility of fragmenting coal into small particles by means of high intensity sound waves might well be examined. Sound waves produce large stress and strain gradients in the propagating medium, especially at high frequencies. In effect, therefore, a high intensity sound wave might function as a novel sort of grinder. If the water in the coal pores could be excited to cavitation, then the erosive forces characteristically accompanying cavitation might quite effectively pulverize the coal. Even if fragmentation of coal by high intensity sound waves proves impractical, cavitation and acoustic streaming might be used to further fragment already small coal particles suspended in a slurry (which could be a coal-oil slurry, see Section IV E 5); here the liquid surrounding the coal (not the liquid in the coal) would be brought to cavitation. In this scheme, acoustic fragmentation would be employed only to reduce particle sizes below the size at which continued mechanical grinding becomes extremely inefficient.

Obviously, any scheme involving use of intense sound waves in a coal utilization process will be impractical if the energy required to produce and transmit the ultrasonic beam is a significant fraction of the expected energy yield from the process. A simple calculation suggests that intense sound waves are not impractical from this standpoint. Suppose, for example, coal particles suspended in an oil slurry could be fragmented to desired very small sizes by means of acoustic waves. Typical usable coal-oil slurries contain about $50 \%$ coal by weight. Therefore, taking the energy content of coaf equal to $10,000 \mathrm{Btu} / \mathrm{lb}$, and using 1.3 $\mathrm{gm} / \mathrm{cm}^{3}$ for the density of coal, the coal energy contained in a volume Ad of this coal-oil slurry will be approximately $2 \times 10^{11}$ Ad ergs in cgs units, where $A$ is interpreted as the area of the acoustic beam and $d$ is the thickness of the layer (presumably about equal to the attenuation distance of the acoustic beam in the slurry) within which coal chunks will be fragmented. The rate of radiation of acqustic energy into the slurry will be $\mathrm{Ap}^{2} / 2_{\mathrm{p}} \mathrm{c}$; where $\mathrm{p}$ is the maximum pressure, $\rho$ is the fluid density, and $c$ is the velocity of sound. Using $p=0.7 \mathrm{gm} / \mathrm{cm}^{3}$ and $c=10^{5}$ $\mathrm{cm} / \mathrm{sec}$, typical values for organic liquids of moderate atomic weight (e.g. n-pentane), the rate of radjation of acoustic energy will be about $7 \times 10^{\circ} \mathrm{A}$ ergs $/ \mathrm{sec}$ for $\mathrm{p}=10^{6}$ dynes $/ \mathrm{cm}^{2}=$ atmospheric pressure, a pressure sufficiently high to cause cavitation in undegassed water at moderate frequencies. Consequently the acoustic energy expended in inducing cavitation should be negligible compared to the energy content of the fragmented coal in the coal-oil slurry, even if $d$ is no more than about one centimeter, the acoustic signal must be maintained for times of the order of a few seconds, and the ultrasonic pressures required are as high as ten times atmospheric. Nevertheless, though this energy comparison seems very favorable, the feasibility of this acoustic fragmentation suggestion ultimately hinges on whether a significant fraction of the acoustic energy dissipated in the slurry will go into cavitation-inducing mechanisms. Further investigation is required.

Uses of Low and Moderate Intensity Sound Waves: Present uses of low and moderate intensity sound waves in applied and basic science, for diagnostic, analytic, and control purposes, are numerous and varied. There are many possible applications of acoustics to various coal utilization industry problems. For instance, measurements of the scattering of acoustic waves in coal can reveal coal inhomogeneities and microstructure. Measurements of acoustic velocities and attenuations might correlate usefully with the aromaticity of coal, and in that case would become a useful tool for rapidly determining 
coal aromaticity and related properties. It is possible that similar measurements might provide a rapid tool for diagnosing the progress of liquefaction in coal liquefying processes. Acoustic tomography is being investigated at the present time (Carson, 1977); successful development of this technique could make it possible to probe for defects within reactors, to visualize the growth of bubbles in fluidized beds, etc. Even if tomography proves to be impractical, the measurement of acoustic scattering at well-chosen frequencies could help to diagnose bubble growth and other phenomena within fluidized beds. Measurements of ultrasonic absorption and dispersion over a wide frequency range, known as ultrasonic relaxation spectroscopy, have been used to study the physical properties of materials as well as reaction kinetics. Moreover, various methods have been devised for extracting information about material properties by making use of acoustic coupling to electromagnetic waves. Such methods include photoacoustic spectroscopy, and acoustic nuclear magnetic resonance.

Many of the just mentioned acoustic applications depend for their effectiveness on the ease with which -- when high intensity acoustic output is not required - the frequencies of acoustic waves can be tuned over very wide bands without much modification of the equipment being used. A possible application of this acoustic frequency band variation is particle size measurement, whose achievement by optical means has been discussed above in subsection IVB 4 . When particle sizes get much larger than about 100 micrometers, particle sizing by optical means can become difficult, in large part because such particles are too large compared to optical wavelengths for sensitive dependence of the scattering on particle size. However, acoustic frequencies easily can be adjusted to yield wavelengths in the range where scattering by particles of 100 micrometers and greater is sensitive to particle size. Therefore, acoustic waves may provide a convenient method of particle sizing over a range of larger particle dimenensions than can be readily sized with analogous optical techniques. The theory of acoustic scattering by small particles is not identical with the Mie scattering theory used to analyze optical scattering, but is closely related, and can be computed for whatever parameters (particle densities, sound speeds, acoustic absorption coefficients) are desired. One difficulty with this acoustic method is that the attenuation of acoustic waves in air and other gases at wave lengths of approximately 50 micrometers is large. An ultrasonic particle detector, purportedly usable for on-line process control monitoring of particles as small as one micron in diameter already has been marketed (Robinson, 1981).

\section{Recommendations-instrumentation and control}

The lack of data necessary for fundamental understanding of advanced fossil energy processes largely is caused by a deficiency in diagnostic instrumentation to make detailed measurements of process behavior, coupled with a lack of access to experimental facilities. Such measurements are essential for model creation and verification as discussed in IVC on the one hand, and are necessary for interpretation of instrument readings and reliable scaleup of the instruments on the other hand. To the end of providing these data and ultimately making possible the control of the advanced fossil energy processes (as discussed in the introduction to this section), we recommend the following:

1. For the purpose of improved process control, present research directed toward increasing our ability to measure -- and thereby monitor -- process parameters under the harsh corrosive environments of coal utilization plants, including temperatures over $1000 \mathrm{C}$ and pressures of 1000 psi, must be expanded. These parameters include, but by no means are limited to: reactor temperatures; mass flow rates and chemical species concentrations in multiphase process streams; particulate numbers and size distributions in gas streams to turbines and to the atmosphere; and fluidized bed levels.

2. For improved efficiency and safety, instrumentation researches must be aimed at techniques permitting real-time monitoring of as many process parameters as possible, especially of reactor temperatures. Often the harsh reactor environments and component inaccessibility will require that this monitoring be accomplished non-invasively as well.

3. In view of the present paucity of reliable instrumentation for coal plant process controls, a systematic evaluation of available 
commercial and developmental instrumentation should be undertaken, with the clearly stated objectives of identifying promising present techniques and encouraging their improvement. Commercial instruments include, but are not limited to, state-of-the-art instruments discussed in this section: acoustic and coriolis flowmeters; optical particulate monitors; thermocouple, sodium line reversal, and optical pyrometer temperature measuring instruments; and spectrographic and infrared gas analysis. Developmental instruments include: acoustic, capacitive, and nuclear devices to measure high temperature multiphase flow rates; electromagnetic and acoustic level measuring instruments; neutron capture gamma ray techniques for on-line analysis of dense phase streams; improved thermocouple sheaths; and laser-based combustion-monitoring instrumentation, including on-line gas analysis.

4. To achieve the reliable process controls needed for efficiency and safety, not only must present instrumentation techniques be improved, but investigations of possible new techniques -- involving physical principles and phenomena hitherto largely ignored in coal process monitoring -- will have to be actively encouraged, even if at first sight highly speculative. These might include reactor temperature measurements via conventional vibrational and rotational band spectroscopy and via laser Raman scattering; reactor temperature measurements inferred from acoustic time domain reflectometry or from Johnson noise; use of crystal thermometers and novel thermocouple elements, e.g., semiconductors; acoustic particulate sizing; and even ultrasonic aids to particulate agglomeration and to coal liquefaction and comminution.

5. To assist the development of new instrumentation and the improvement of presently available instruments, the full range of electromagnetic and acoustic radiations from reactor process components should be carefully studied for possible extraction of information on temperature, composition, etc., bearing in mind that excursions of these radiations can indicate deviations from normal operation. In many cases, research on novel signal processing techniques will be a necessary part of such studies.

6. Reactor modeling efforts, which up to now have been largely ignored, and have been comparatively rudimentary when undertaken, must be greatly increased, with the objectives (inter alia): of improving our abilities to interpret and usefully employ instrumentation readings; and of finding indirect measures of reactor temperatures and other important reactor process parameters which are difficult to measure directly.

7. Dedicated test facilities, for instrumentation research and for process model development and verification (using these instruments) must be made available. These include facilities for the study of multiphase flow as well as combustion and conversion characteristics. It is recognized that some facilities exist, but additional or expanded facilities will be necessary. While operating plants can be used for some activities of this kind, these facilities presently are available only on a non-interference basis and are not adequately instrumented for collection of meaningful process data.

\section{References}

Allen, T., 1975, Particle Size Measurements 2nd edition, Chapman and Hall, Ltd.

American Society for Testing and Materials, 1977, Standard Method for Sampling Stacks for Particulate Matter, Designation D2928-71, in Annual Book of ASTM Standards, Philadelphia, PA, pp. 592-618.

Argonne, 1980, "Overview of Coal Conversion Process Instrumentation", May 1980, Argonne National Laboratory Report, ANL-FE-49628-TM01.

Bachalo, W. D., 1980, Applied Optics 19, 363.

Bachalo, W. D., J. Geffken and G. Weth, 1978, "On-Line Particle Monitoring Instrumentation", Proc. of Symp. on Instrumentation and Control for Fossil Demonstration Plants, ANL-78-62-CONF 780656, Argonne National Laboratory Report.

Ballard, Evan, 1981, Los Alamos National Laboratory, Private Communication.

Basedow, Ebert, 1979, "Effects of Mechanical Stress on the Reactivity of Polymers: Activation of Acid Hydrolysis of Dextran by Ultrasound", Polym. Bull. (Germany) Volume 1, pp. 299-306.

Baucum, W. E., 1979, "Status of the Evaluation of a Coriolis Effect Mass Flow Meter for Dense Phase Coal Flows", in the Proceedings of the 1979 Symposium on Instrumentation and Control for Fossil 
Energy Processes, ANL-79-62, CONF 790855, held August 20-22, Denver, CO.

Billings, C. E., J. Wilder, 1970, Handbook of Fabric Filter Technology, GCA Inc., Bedford, MA.

Bozorgmanesh, H., et al, 1980, "Operational Characteristics of the On-Line Sulfur in Coal Analyzer", in the Proceedings of the 1980 Symposium on Instrumentation and Control for Fossil Energy. Process, ANL-80-62, CONF 810607, held June 8-10, 1981, in San Francisco, CA, to be published late in 1981.

Brown, D. R., et al, 1978, "Moisture Determination in Coal: Survey of Techniques" in the Proceedings of the 1978 Symposium on Instrumentation and Control for Fossil Demonstration Plants, ANL-78-62, CONF 780656, held June 19-21, 1978, Newport Beach, CA.

Bump, T. R., 1979, "Design and Initial Testing of High Temperature/Pressure Samplers" in the Proceedings of the 1979 Symposium on Instrumentation and Control for Fossil Energy Processes, ANL-79-62, CONF 790855, held August 20-21, 1979, Denver, CO.

Carlin, Benson, 1964, "Applications of Ultrasonic Waves", in Physical Acoustics, edited by W. P. Mason, Vol. I, Part B, Academic Press.

Carson, E., 1977, Med. Phys. 4, 302.

Cekorich, A., 1979, "Development of an Elemental Analyzer for Coal, Oil and Similar Bulk Streams" in the Proceedings of the 1979 Symposium on Instrumentation and Control for Fossil Energy Processes, ANL-79-62, CONF 790855, held August 20-22, 1979, Denver, Co.

Chigier, N. A., 1976, Prog. Energy Combustion Sci., 2, 97.

Chigier, N.A., 1977, Prog. Energy Combustion Sci., 3, 175 .

Cooke, D. D., M. Kerker, 1975, Appl. Optics 14, 734 .

Cummins, H. A. E. R. Pike, editors, 1974, Photon Correlation and Light Beating Spectroscopy, Plenum Press.

Cummins, H. A., E. R. Pike, editors, 1977 , Photon Correlation Spectroscopy and Velocimetry", Plenum Press.

Ellingson, W. A., 1980, Materials Technology for Coal-Conversion Processes, Progress Report for April-June, 1980, Argonne National Laboratory Report ANL-80-93.

EPRI, Electric Power Research Institute, 1979, Private Communication.
Farmer, W. M., 1973, "The Interferometric Observation of Dynamic Particle Size, Velocity and Number Density", Ph.D. thesis, U. Tennessee.

Farmer, W. M., 1976, Appl. Optics 15, 1984.

Flynn, H. G., 1964, "Physics of Acoustic Cavitation in Liquids", in Physical Acoustics, edited by W. P. Mason, Vol. I, Part B, Academic Press, pp. 57-152.

Frederikse, H. P. R., 1963, "Properties of Semiconductors", in American Institute of Physics Handbook, McGraw Hill, pp. 9-53 to 9-54.

Gozani, T., O. J. Tassicker, R. Bevan, 1979, "Advanced Techniques and Instrumentation for Real Time On-Line and Laboratory Analysis of Coal" in the Proceedings of the 1979 Symposium on Instrumentation and Control for Fossil Energy Processes, ANL-79-62, CONF 790855, held August 20-22, 1979, Denver, CO.

Hartley, D. L., 1975, "The Role of Physics in Combustion", American Institute of Physics.

Herzenberg, C. L., 1979, "Use of Small Accelerators in Coal Analysis and Coal Slurry Flow Measurements", in IEEE Transactions on Nuclear Science, NS-26, pp. 1568-1573.

Herzenberg, C. L., et a1, 1978, "Process Stream Compositional Analysis in Advanced Coal Utilization Plants Using Neutron-Induced Gamma Spectrometry" in the Proceedings of the 1978 Symposium on Instrumentation and Control for Fossil Demonstration Plants, ANL-78-62, CONF 780656, held June 19-21, 1978, Newport Beach, CA.

Herzenberg, C. L., K. E. Griggs, R. F. Henry, W. F. Podolski, 1981, "A Study on Instrumentation Needs for Process Control and Safety in Coal Fluidized-Bed Combustion Systems", Argonne National Laboratory Report ANL/CEN/FE-80-12.

Herzfeld, C. M., editor, 1962, "Temperature: Its Mesurement and Control in Science and Industry", Vol. 3, Reinhold.

Karplus, H. B., A. C. Raptis, 1981 , "Development and Testing of A High-Temperature Acoustic Doppler Flowmeter", ANL-FE-469609-TM01, to be published in May, 1981.

Kinzi, P. A., 1973, "Thermocouple Temperature Measurement", (Wiley) and references under the heading "Thermocouples with Graphite or Carbon", p. 208. 
Knollenberg, R. G., 1979a, "An In-Stack Fine Particle Spectrometer: A Discussion of Its Design and Development", Advances in Particle Sampling and Measurement, U.S. EPA Report EPA-600/7-79-065, p. 169.

Knollenberg, R. G., 1979b, "Single Particle Light Scattering spectrometers", in Aerosol Measurement, edited by D. A. Lundgren, F. S. Harris, Jr., W. H. Marlow, M. Lippmann, W. E. Clark, M. D. Durham, pub. by Univ. of Fla. Press, Gainesville.

Lamb, G. E. R., P. A. Costanza, 1980, Textile Res. J. 50, 661.

Liu, B. Y. H., D. Y. Pui, A. Kapadia, 1979, "Electrical Aerosol Analyzer: History, Principle and Data Reduction", in Aerosol Measurement, edited by D. A. Lundgren, F. S. Harris, Jr., W. H. Marlow, M. Lippmann, W. E. Clark, M. D. Durham, pub. by Univ. of Fla. Press, Gainesville.

Lundgren, D. A., F. S. Harris, Jr., W. H. Marlow, M. Lippman, W. E. Clark, M. D. Durham, editors, 1979 , Aerosol Measurement, pub. by Univ. of Fla. Press, Gainesville.

Managan, W. W., J. J. Eichholz, R. W. Doering, 1978, "Field Test of a Capaitative Transduce for Density/Velocity (Mass Flow) Measurement on the HYGAS Pilot Plant Solvent/Coal Feedline" in the Proceedings of the 1978 Symposium on Instrumentation and Control for Fossil. Demonstration Plants, ANL-78-62, CONF 780656, held June 19-2l, 1978, Newport Beach, CA.

Marple, V. A., K. Willeke, 1979, "Inertial Impactors", in Aerosol Measurement edited by D. A. Lundgren, F. S. Harris, Jr., W. H. Marlow, M. Lippmann, W. E. Clark, M. D. Durham, Univ. of Fla. Press, Gainesville.

Mazumder, M. K., R. G. Renninger, T. H. Chang, R. W. Raible, W. G. Hood, R. E. Ware, M. A. Smith, 1981, "Simultaneous Measurement of Aerodynamic Size and Electrostatic Charge of Aerosol Particles in Real Time and on Single Particle Basis", Proc. of Third Symposium on the Transfer and Utilization of Particulate Control Technology, ed. by F. P. Vendittle, pub. by Denver Research Institute, Orlando, Fla.

McLean, W. J., D. R. Hardesty, J. H. Pohl, 1980, "Direct Observation of Pulverized Coal Particles in a Combustion Environment", Central states Section
Meting, The Combustion Institute, Louisiana State Univ., paper 80-11. Sandia Laboratories Report.

O'Fallon, N. M., R. W. Doering, C. P. Leiter, 1981, "Review of Instrumentation Needs for Process Control and Safety in Advanced Fossil Energy Processes", Argonne National Laboratory Report, ANL-FE-49628-TM02, to be published in May, 1981 .

O'Fallon, N. M., et al, 1976, "A Study on the State-of-the-Art of Instrumentation for Process Control and Safety in Large-Scale Coal Gasification, Liquefaction, and Fluidized-Bed Combustion Systems", Argonne National Laboratory Report, ANL-76-4.

Papadakis, Emmanuel M., 1976, "Ultrasonic Velocity and Attenuation: Measurement Methods with Industrial Applications", in Physical Acoustics, Volume XII, Academic Press.

Penny, G. W., 1977, "Using Electrostatic Forces to Reduce Pressure Drop in Fabric Filters", Powder Separation 18, 111 , "Electrostatic Effects in Fabric Filtration", EPA-600/7-78-142a EPA, Research Triangle Park, N.C.

Pitcher, N. D., 1977, "Bi-Gas High Temperature Thermocouples Design Considerations and Operating Experience" in the Proceedings of the 1977 Symposium on Instrumentation and Process Control for Fossil Demonstration Plants, ANL-78-7, CONF 770729, held July 13-15, 1977, Chicago, III.

Plumb, H. H., editor, 1972, "Temperature: Its Measurement and Control in Science and Industry", Volume 4, Reinhold.

Raptis, A. C., et a 1, 1978, "HYGAS Experiment on the Feasibility of Acoustic/Ultrasonic Flowmeters" in the Proceedings of the 1978 Symposium on Instrumentation and Control for Fossil Demonstration Plants, ANL-78-62, CONF 780656, held June 19-21, 1978, Newport Beach, CA.

Robinson, A. L., 1981, Science 212, 146.

Sessler, G. M., 1968, "Acoust $\overline{i c}$ and Plasma Waves in Ionized Gases", in Physical Acoustics, edited by W. P. Mason and R. N. Thurston, Volume IV, Part B, Academic Press.

Sheen, S. H., A. C. Raptis, 1979, "HYGAS Coal-Slurry Mass-Flow Measurements Using Ultrasonic Cross-Correlation Techniques, Argonne National Laboratory Report, 
ANL-FE-49622-TM07.

Shewmon, P. G., 1978, "Materials in Coal Utilization: Mechanical and Electrical Properties" in Scientific Problems of Coal Utilization, Conference Proceedings edited by B. R. Cooper (Technical Information Center, Dept. of Energy).

Sinclair, D., R. J. Countess, B. Y. H. Liu, and D. Y. H. Pui, in Aerosol Measurement, edited by D. A. Lundgren, F. S. Harris, $\mathrm{Jr} ., \mathrm{W} . \mathrm{H}$. Marlow, M. Lippman, W. E. Clark, M. D. Durham, pub. by Univ. of Fla. Press, Gainesville.

Smith, A., et al, 1979, "Acoustic Monitoring for Leak Detection in Pressurized Water Reactors", in Acoustic Emission Monitoring of Pressurized Systems, American Society for Testing and Materials Special Technical Publication ASTM 167.

Smith, A., 1981, Argonne National Laboratory, Private Communication.

Spanner, J. C., 1979, "Acoustic Emission Some Examples of Increasing Industrial Maturity" in Acoustic Emission Monitoring of Pressurized Systems, American Society for Testing and Materials Special Technical Publication ASTM 167.

Sullivan, J. A., Reed J. Jenson, 1981 , "Laser-Based Spectroscopic Techniques for Measurement of Containment Species in Coal-Gasification Product Streams", presented at the American Institute of Chemical Engineering Meeting, Houston, TX.

Stewart, R. P., A. W. Hall, J. W. Martin, W. L. Farrior, A. M. Poston, 1974, "Nuclear Meter for Monitoring the Sulfur Content of Coal Streams", Bureau of Mines Technical Progress Report 74.

Swithenbank, J., J. M. Beer, D. S. Taylor, D. Abbot, G. C. McCreath, 1977, "A Laser Diagnostic Technique for the Measurement of Droplet and Particle Size Distributions", AIAA paper No. 76-69, A IA 14 th Aerospace Sciences Meeting, Washington, D.C., January 26-28, L976.
Also published in Experimental Diagnostics in Gas Phase Combustion Engines, Progress in Astronautics and Aeronautics, editor B. T. Zinn, 53, 421.

Symposia on Instrumentation and Control for Fossil Eneergy Processes, Proc. Available from NTIS, 5285 Port Royal Road, Springfield, VA, 226161.

ANL-78-7, CONF 770729, 1977

ANL-78-62 CONF 780656, 1978

ANL-79-62 CONF 790855, 1979

ANL-80-62 CONF 800602, 1980

ANL-81-62 CONF 810607, 1981

U.S. Environmental Protection Agency, 1976, "Standards of Performance for New Stationary Sources", Federal Register, 42(187), pp. 42020-42028.

U.S. Environmental Protection Agency, 1977, "Standards of Performance for New Stationary Sources", Federal Register, 43(160), 41776.

Volk, M., W. Moroz, 1976 , "Sonic Agglomeration of Aerosol Particles" in Water, Air, and Soil Pollution, (Netherlands), Vol. 5, pp. 319-334.

Weiner, B. B., 1979, "Particle and Spray Sizing Using Laser Diffraction", Soc. Photo-Optical Instr. Eng. Vol. 170 , Optics in Quality Assurance II, p. 53.

Whitby, K. T., W. E. Clark, 1966, Tellus, $18,573$.

Whitby, K. T., K. Willeke, 1979, "Single Particle Optical Counters: Principles and Field Use", in Aerosol Measurement, edited by D. A. Lundgren, F. S. Harris, Jr., W. H. Marlow, M. Lippman, W. E. Clark, M. D. Durham, pub. by Univ. of Fla. Press, Gainesville.

Zweibaum, F. M., 1978, "Integrated Radiometer System for Gasifier Temperature Measurement" in the Proceedings of the 1978 Symposium on Instrumentation and Control for Fossil Demonstration Plants, ANL-78-62, CONF 780656, held June 19-21, 1978, Newport Beach, CA. 


\section{C. MODELING AND FLOW THEORY}

\section{Introduction}

Modeling of coal conversion processes is a research category with high potential for producing results which can dramatically affect the clean, safe utilization of coal. It is an area, however, into which many physicists have been reluctant to enter because of both natural inclination and background. There are, nevertheless, challenging and interesting problems to which physicists could contribute.

Briefly, we find three major areas in which physicists could have significant impact: (a) in the construction of particulate burn models; (b) in the linkage between modeling and instrumentation, and (c) last, but by no means the least, in fundamental advances in the theory of multiphase flow phenomena.

Here, we provide an overview of the various theoretical aspects of coal utilization processes with emphasis on those areas where, in our opinion, physicists would feel "at home" and could provide especially useful contributions. An example is the relationship between radiative transport and temperature equilibrium in combustion and combustor reactors. On the other hand, we recognize that there are other aspects, such as direct liquefaction reactor system modeling, which are more suitably addressed by chemical engineers, because these aspects are totally permeated with details of organic chemistry which are not part of most physicists' working tools. We hope that our overview provides enough guidance through this maze of varied topics to permit physicists to identify the challenges and research opportunities available to them.

The major motivation for research in theoretical modeling in coal utilization today is the need for more detailed understanding of the processes and outputs. Realistic models acquire increased importance when details are significant. The new significance of details is illustrated by the following synopsis of problems currently faced by operators of plants utilizing direct combustion of coal for the generation of electrical power. First, a rapidly changing energy supply situation has forced operators of combustion equipment designed for liquid or gaseous fuels to consider, or in some cases face a requirement for, switching to solid fuels. The operator must then determine whether to use conventionally pulverized coal after incorporating dramatic equipment changes, to use the existing equipment at reduced loads, or to fire the solid fuel in an unconventional mode (ultra-fine particles, oil slurries, etc.). The operator will find the basic technical information on which to base this decision essentially unavailable. Furthermore, predictive tools necessary to extrapolate experience gleaned from one coal type to another are also non-existent. In addition, environmental considerations require combustion modification for pollutant emission abatement. For example, nitrogen oxide (NOX) abatement can be accomplished economically only through prevention of their formation, and not through stack removal. As discussed below, one of the major recent accomplishments of combustion and flow modeling has been in providing sufficient understanding of process chemistry to devise a practical way of reducing NOX production in coal combustion. The essential point brought out by this advance is that careful and correct particulate burn modeling and characterization are necessary in order to achieve a desired change without detracting from the original purpose, namely the production of electrical power.

Modeling of coal conversion processes can be loosely classified into three overlappping categories of scale, necessitating rather different types of theoretical considerations. These are (a) the particulate level, i.e. combustion or reaction of single coal particles; (b) zone modeling of an ensemble of coal particles under uniform conditions, or conditions which represent some average behavior; and (c) macroscopic, or reactor scale, modeling of whole coal reaction chambers of various kinds. Each successive scale subsumes the previous one, but presents its own unique problems in modeling. We discuss examples from each category in separate subsections below.

As will be seen, all of these modeling efforts are linked to the flow conditions of the materials involved, requiring details of multi-phase flow. This is an important topic which pervades all modeling efforts, and one in which theoretical progress is sorely needed. We discuss this general topic separately in Section IV C-5, because it deserves special emphasis as an area in which physicists particularly can contribute.

The above classification scheme is not meant to discount the importance of theoretical modeling of free molecule reactions in coal processes. Such processes form an important 
part of the chain of consideration for certain output products; we briefly address this topic in the following part of this Introduction.

\section{Free molecule reactions}

As we will discuss in Section IV $\mathrm{C}-3$, one of the mechanisms for Nox production is the so-called "thermal" NOX mechanism, the $\mathrm{O}_{2}-\mathrm{N}_{2}$ reactions in gas phase. Such reactions are amenable to theoretical treatment. In this particular case the reaction is reasonably well understood. Reaction rate modeling can help, however, in assessing the role of intermediate molecular processes which occur during coal combustion or gasification. Many of these intermediate steps are currently not understood. Yet, in some cases, production of "minor" constituent pollutants may be controlled by some such intermediate step. An example of this is the role of the $\mathrm{OH}$ radical in combustion; here a more believable theoretical model would be extremely useful because the experimental investigation is difficult.

A full quantum mechanical treatment of polyatomic reactions at the elevated temperatures involved in these processes is intractible and probably not useful. However, semiclassical models in which the interatomic potentials are specified from spectral information and the motions of the atoms are treated classically are now feasible on moderate sized computers (Lamb, 1980, Poppe, 1980). With this experimentally determined characterization of the interaction potentials, the resultant motions of the constituents are calculated and averaged over initial conditions. Relative probabilities for various rearrangements of the atoms can then be calculated, leading to quantitative estimates of reaction probabilities. This is a new and promising area of research, and the connection to quantum theory via the available phase space is also being investigated (Reinhart, 1980). classical estimates of ionization and excitation rates by charged particle impact have long been successfully used in plasma physics, and the classical description lends itself well to characterization of the reaction probability as a function of temperature. These successes in plasma physics suggest that this type of model may provide useful expressions for organization of global experimental data, and for charactization of the contributions of intermediate steps not easily accessible experimentally. The need to understand more details would be well served by successful research along these lines.

\section{Modeling at the particulate level}

In this section we briefly describe coal particle modeling. This topic is central to all existing combustion and gasification schemes. Related topics such as surface reactions and soot formation are included. This is an area which deserves emphasis as one of special interest to physicists.

When a small coal particle is immersed in a hot, oxidant-containing gas environment, any water present is quickly desorbed. This is followed by volatilization of the aliphatic carbon compounds leaving a porous, solid mass called char (Solomon, 1980). The volatilized carbon compounds oxidize rapidly, and, if the temperature and oxidant concentration are high enough, completely. The behavior of the char consists of a more or less rapid reduction in size, also depending on the gas temperature, particle size, and oxidant concentration.

The modeling of particulate behavior under these conditions consists of: (a) identifying the physical processes important to volatilization, temperature determination, and size reduction; (b) correctly incorporating these sources and sinks in the energy, momentum and mass continuity equations (see Section IV C-5) with appropriate boundary conditions, and (c) solving the resulting coupled equations for particle temperature and radius as a function of time. Analytical solutions or approximate representations are highly desirable, because this description can then be used as a part of the macroscopic modeling of reactor systems. A high level of sophistication is called for in successful parameterization of particulate burn models if they are to be simple enough to be useful. An especially thorny problem encountered is the handing of the mineral matter in these models, because melting temperatures for the mineral matter are often reached before burn out, and the distribution of the energy among non-reactive minerals affects oxidant transport, causing slag to cover the particle surface. It is also important to have good characterization of reaction rates. Most particulate models to date deal only with gross aspects of energy release and size reduction.

Combustion is the term used to describe the rapid self-sustaining exoergic oxidation of 
carbon compounds, and arises as a result of heat loss and generation through reactions (Mulcahy and Smith 1969, Mulcahy 1977). At low gas temperatures, cooling loss rates (primarily convective) are faster than energy generation rates, and the equilibrium reached is one in which the particle temperature is only slightly higher than that of the environment, i.e., no combustion occurs. At high temperatures, the energy generation reaction rates are large, and a new equilibrium is reached at particle temperatures which are much higher than the ambient, and which are controlled by the rate of reaction and radiation transport. The char particles, therefore, may or may not combust, depending on size and temperature (Ubhayakar and Williams, 1976), again provided that the oxidant concentrations are adequate. If they do undergo combustion, size reduction is rapid ( 0.1 seconds burning time for $10 \mu \mathrm{m}$ particles at $2000 \mathrm{~K})$. Thus the total dwell time necessary for complete combustion of small particles in a reactor is not large, a typical number being one second.

The volatilization of the aliphatic compounds mentioned above does not occur isotropically; instead recent experiments (Hardesty, 1980) indicate that these volatiles are emitted in jet-like spurts. This indicates that the aliphatic compounds may have been gasified in internal pores, and finally emerge as a jet when the pressure becomes large enough (Hardesty 1980). Coal particles with low content of aliphatic carbon compound do not exhibit this jetting. Modeling of this behavior has not been attempted. This effect needs to be examined theoretically, and determination made as to how it can be incorporated in particulate burn models.

At high temperatures, the volatiles combust completely, producing $\mathrm{CO}_{2}$ and $\mathrm{H}_{2} \mathrm{O}$ only. If the temperature is reduced sufficiently, however, the combustion of the volatiles is not complete, and instead these compounds form soot particles. The nucleation of the soot particles is not understood; it is possible that the models discussed in the Introduction could be used to provide some understanding of this situation.

As the above description indicates, surface reactions are very important in the modeling of particulate burning. The reactions being discussed here are qualitatively different from those discussed in Section IV F, associated with catalysis. The latter are site-specific; only certain lattice sites can produce the reactions. In the combustion of a coal particle, all of the atoms are eventually involved in a reaction, and a global reaction description is appropriate. Theoretical guidance is needed for structuring the terms in the particulate models. Chemisorption theory may provide one vehicle for doing this.

Chemisorption of atoms or molecules on surfaces is a subject which is currently of great theoretical interest (Smith 1980). Sometimes the substrate on which the adsorption takes place is treated as a generalized "jellium" (Gunnarson, 1977, Lang 1978). This is primarily applicable to simple (i.e. nontransition) metals. Given the global nature of the reaction rates of interest in particle combustion, it may be that some such description could be useful in these studies. Strictly speaking a jellium description applies only when behavior is determined by average electron behavior; while, in fact, adsorption is expected to occur primarily at selective sites in the coal. Nevertheless, the details of the reaction sites are likely to vary widely, it may be possible to represent them in terms of some generalized description representing an "average" site in the spirit of the jellium calculation.

The standard adsorption theories deal with the bonding potential of an atom on a surface by means of calculation of the electronic potential produced by the substrate and atomic electrons, either via, a model hamiltonian or using self-consistent field methods. It may be possible to use these theories as a basis for modeling surface reactions, i.e., the potentials calculated from such treatments could be used to estimate activation energies and reaction rates. This area appears to hold substantial promise of theoretical progress.

As in consideration of free molecule reactions, the important requirement for models appropriate to coal burning is to include the dominant physical effects. These models should be based on specific detailed calculations where possible, but the specifics of the problems are in general so complicated that completely rigorous calculations do not offer much potential, particularly because of the high temperature environment in which they must be applied. On the other hand, a simplified model containing the essential physically important ingredients, may predict trends, such as temperature dependence, which can be readily used.

Re-examination of the existing particulate models is necessary for further progress at this time. Indeed, modeling at the particular 
level is a subtle and sophisticated area of research which offers substantial challenges, because of the necessity to balance simplicity of description, inclusion of all the important physical phenomena, and retention of just enough detail to extract the information on relevant outputs of a coal combustion system.

\section{Zone models*}

Two types of reactor models have been used historically. In the simplest, or "zero dimensional" type, the entire reactor is treated as a single zone and the chemical reactions occuring are simply treated as energy sources or sinks. Mass, energy and species balances are then used to infer mean temperatures and outputs. In the second type of reactor model, geometrical symmetry is used to consider the reactor as a coupled series of sections, or zones, with energy, momentum and mass balances being used to determine one dimensional profiles of the important variables.

Essentially all modeling of industrial furnaces and utility boilers has historically been zone type modeling. These reactors are typically very large volume reaction chambers, in which pulverized (50-200 $\mu$ size) coal is sprayed into (usually also premixed with) a hot air stream under combustion sustaining conditions. The flow rates are high enough so that swirling, turbulent flow occurs. The swirling and turbulence, rather than being circumstantial or bothersome, are essential features of the design. They are required to provide the air-fuel mixing for complete combustion. Most utility burners are quite efficient, with better than $90 \%$ of the energy available in the fuel being recovered as steam energy. Other types of industrial burners are the fixed bed or traveling bed furnaces in which the coal is in a layer and hot air is forced through the bed (Beer 1980).

The modeling is accomplished via the concept of a "well stirred reactor" (Longwell and Weiss 1955) model. These models ignore the details of turbulent fluid dynamics and deal with spatially averaged properties of the combustion system, either in layers coupled together, or for the reactor as a whole. The "zero dimensional" models are reviewed by Mellor (1972). The appropriate averaging of the differential equations involved is

*Also called lumped parameter models. discussed in Section IV C-5. In these models, global* chemical reactions are used to describe the combustion.

In general, these simple models have been successful in accounting for energy production, mean temperatures, major constituents of the gaseous outputs, and other averaged parameters of the reactor. This is due primarily to the fact that furnaces and utility boilers are operated under conditions which lead to full stoichiometric combustion of the fuel. Until recently this has been considered a sufficient understanding of the process.

More elaborate one-dimensional zone modeling for steady flows has also been done. As discussed in Section IV C-5, such models require averaging of the general three-dimensional equations over two space dimensions (Williams 1972; Ishii 1975). Many early models used heuristic forms of the appropriate equations and took into account turbulence via empirical diffusion and viscosity coefficients. Usually radiative energy transfer is ignored or treated only grossly, but some zone models have included radiative transfer (Lowe, et al. 1977). Twoand three-dimensional modeling of turbulent flow will be discussed later.

The new constraints involving knowledge and control of pollutant emissions have turned up inherent weaknesses of earlier simpler models. It has become important to understand details of the production of minor product constituents such as NOX and oxides of sulfur (SOX), so that emission control can be effected. In fact, the control of nitrogen oxides can be considered one of the practical triumphs of modeling. It has been recognized that there are two sources of nitrogen oxides in coal combustion: 1) release of oxides from the fuel-bound nitrogen, and 2) the breakup and oxidation of the nitrogen molecules in the hot air used as oxidant. This latter mechanism is termed thermal NOX production. The fuel-bound NOX source is potentially four to six times larger than the thermal NOX production, under ordinary circumstances. However, if the volatilization is accomplished at high temperature under oxygen poor conditions, the amount of fuel-bound NOX is dramatically

* Global reactions are those in which initial and final species are specified, assuming that any intermediate steps are fast enough and concentrations adequate to produce the stated results. For minor constituents, this assumption constitutes a serious drawback. 
reduced (Wendt 1980). Figure IV C-1 shows this effect of experimental data from a test facility (Gershman 1977).

The figure shows experimental data for the release of nitrogen oxides as a function of fuel to oxygen ratio, relative to the amount required for full stoichiometric combustion of the fuel (equivalence ratio of 1.0). The data indicate that as the oxygen concentration is reduced, the production of NOX decreases, approaching the value associated solely with thermal NOX production. Various flow conditions ("swirls") are shown, but all show the same general trend.

Modeling of various types of feed configuration for furnaces has shown that control of gas dynamics can alter the production of NOX substantially. These developments have led to more careful examination of the details of particulate combustion (Wendt 1978, Blair 1977, Levy 1980, Song 1980), both for chars and volatiles. "POX" (small particulate) production is also being investigated (Neville 1980).

Better understanding of coal combustion systems, both at the microscopic and macroscopic levels, is clearly necessary for further advances in reduction of pollutants consistent with maximization of energy production. Additional, more careful modeling

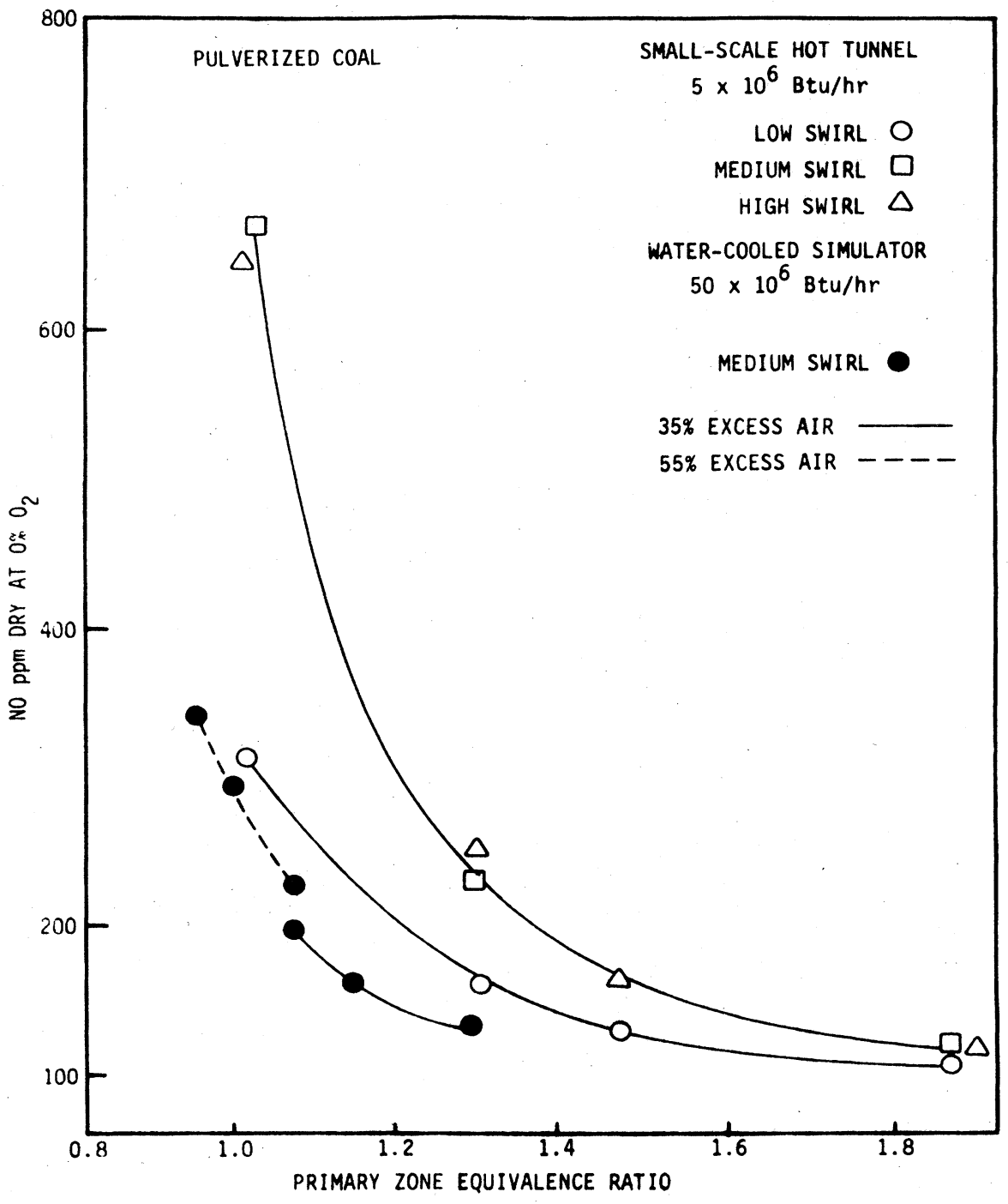

FIG. IVC-1. Reduction of $\mathrm{NO}_{x}$ emissions possible through flame aerodynamic control. Resultant NO concentration (in parts per million) is plotted as a function of the fuel to oxygen ratio, referred to stoichiometric values. As the oxygen concentration is reduced, the NO emission approaches the thermal NO value (after Gershmann et al., 1977). 
is a key ingredient in this understanding. The reduction of sulfur emissions is another constraint, and has also been examined within the existing models. Unfortunately, no simple alterations of combustion conditions have been found which have dramatic effects on this emission, due, of course, entirely to fuel sulfur. As is well known, currently these emissions must be removed from the exhaust gases. Advanced systems for utility boilers deal with this problem by using a fluidized bed combustor (FBC) (similar to the fluidized bed gasifiers described in the Technology Primer -- Section IV A) using coal particles mixed with limestone particles. The lime, or other agent, absorbs the sulfur gases after emission from the coal particles. The FBC differs from the gasifiers in operation in its temperature and pressure conditions, because full combustion, rather than particular gas product production, is the desired result. Zone models for such systems have also been constructed (Sarofim and Beer 1978).

\section{Example of modeling at the coal reactor level}

While most aspects of modeling at the reactor level are necessarily intricately involved in the engineering of the reactor, there are aspects of this problem which could benefit from, and provide challenges, to the physicist. One of these deals with appropriate instrumentation. In particular, there is a close coupling between the need for more detail, model improvement, the appropriate instrumentation necessitated by this need, and the potential role of the physicist. It is probably reasonable to say that the physicist's involvement at the reactor level of modeling would be as a team member. His or her role would lie primarily in critical examination of assumptions used in modeling the physicial phenomena occurring and devising instrumentation appropriate to model verification.

Since it is well studied, and since it is the best defined of the various cases previously cited, the modeling of a fixed-bed gasifier will be followed in a little more detail. The methodology which follows is that of Yoon et al. (1978). They assume a one-dimensional coal gasifier with a steady state coal bed and a counter-current gas flow in thermal equilibrium with it.

They model the chemical reactions involved using the following global reactions:

$$
\begin{aligned}
& \text { (C) }+\mathrm{H}_{2} \mathrm{O} \rightleftarrows \mathrm{CO}+\mathrm{H}_{2} \quad \text { (a) } \\
& \text { (C) }+\mathrm{CO}_{2} \vec{z} 2 \mathrm{CO} 2 \text { (b) } \\
& \text { (c) }+2 \mathrm{H}_{2}^{2} \underset{\mathrm{t}}{\mathrm{t}} \mathrm{CH}_{4} \text { (c) } \\
& \gamma(\mathrm{C})+\mathrm{O}_{2}^{2} \rightarrow 2(\gamma-1) \mathrm{CO}+(2-\gamma) \mathrm{CO}_{2} \text { (d) } \\
& \mathrm{CO}+\mathrm{H}_{2} \mathrm{O}^{2} \rightarrow \mathrm{CO}_{2}+\mathrm{H}_{2}
\end{aligned}
$$

where (C) indicates that carbon in the solid is the reactant; and is temperature dependent (Arthur, 1951), but assumed to be a constant in the Yoon et al. (1978) work. Chemically limited reaction rates are specified by the Arrhenius expression in terms of an "activation energy" $\Delta E$ and a rate constant $\mathrm{K}$ for each of the above reactions (labeled by $\alpha$ ).

$$
\mathrm{K}_{\alpha}=\mathrm{K}_{\alpha}^{\circ} \mathrm{e}^{-\Delta \mathrm{E}_{\alpha} / \mathrm{kT}}
$$

Back reactions (i.e., right to left as written) are accounted for by assuming near equilibrium conditions and an appropriate equilibrium constant. The actual or "apparent" reaction rates include terms for intra-particle diffusion and dependence on an empirically determined turbulent transport coefficient in the gas phase. With distance along the reactor as the independent variable, steady state differential equations are then obtained for the temperature and concentrations of the reacting species, using conservation of mass and energy (including linearized radiative transfer).

These differential equations are invariably "stiff", a term used to describe equations whose dependent variables have widely varying time constants. Such equations present well known difficulties for numerical methods, and must be appropriately handled. In addition, the effects of mineral content, or ash, must be accounted for in terms of some, usually simple model.

Finally, in a complete model, the effect of pyrolysis must be accounted for. In this simple kind of model, additivity of gasification and pyrolysis products is assumed.

Some results of this kind of model are given as Figure IV C-2 (Kosky 1978). Figure IV C-2 shows a composite view of a typical calculaton for local reaction temperature (equal between gas and solid) as a function of position in the gasifier. Also for comparision, the physical model of the 
gasifier is drawn to scale, indicating the relative sizes of the various zones in which dominant processes occur. Gasifier modelers should beware, however, that results can be very dependent on detailed assumptions. Several conclusions can be drawn about modeling gasifiers, the most important of which is that the details of these models are obscure, and the accurate prediction of local effects is very difficult.

\section{Multiphase flow modeling}

Multiphase matter flows are conceptually very difficult to model. These difficulties are apparent even in their qualitative behavior as described in the Technology Primer of Section IV A. No model currently exists which can exhibit all of the modes of behavior or flow regimes that are observed; in fact, there are no successful models at all for some of the regimes. Furthermore, no completely successful scheme exists for determining the conditions for transition from one regime to another.

Basic instantaneous equations of motion for a two-phase situation can be written in terms of continuum field equations for each phase, constitutive equations within regions containing only one phase, and "jump conditions" or boundary conditions between the two phases at the interfaces. The field equations can be compactly written as a generalized conservation equation (Ishii, 1975) for each phase, labeled by subscript $k$.
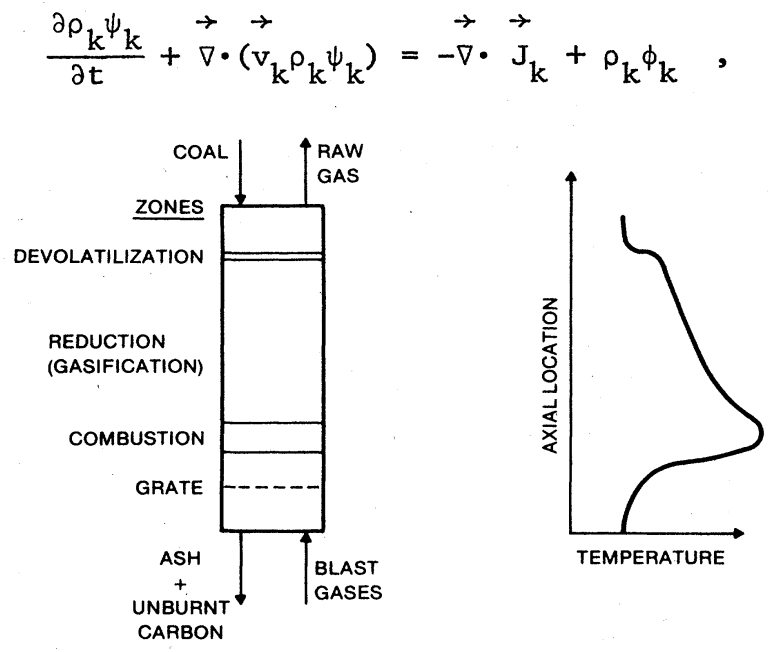

FIG. IVC-2. Gasifier model (after Kosky and Floess, 1980). where $\rho_{k}$ is the instanteous density of the $k^{\text {th }}$ phase, $\psi_{k}$ the general field quantity of interest, $\vec{\nabla}$ the instantaneous velocity. 'The right hand $\mathrm{side}$ terms represent surface fluxes and volumes sources, respectively. The equations governing the rate of change of mass, momentum and energy equations then follow if we use for $\psi_{k}, \vec{J}_{k}$ and $\phi_{k}$ the
following:

(a) for the continuity equation, set

$$
\psi_{\mathrm{k}}=1, \phi_{\mathrm{k}}=0, \overrightarrow{\mathrm{J}}_{\mathrm{k}}=0 \text {; }
$$

(b) for the equations of motion, set

$$
\psi_{\mathrm{k}}=\overrightarrow{\mathrm{v}}_{\mathrm{k}}, \overleftrightarrow{\mathrm{J}_{\mathrm{k}}}=\mathrm{p}_{\mathrm{k}}^{\overleftrightarrow{I}}-{\overleftrightarrow{\tau_{k}}}_{\mathrm{t}}=\overleftrightarrow{\mathrm{T}}_{\mathrm{k}}, \phi_{\mathrm{k}}=\overrightarrow{\mathrm{g}}_{\mathrm{k}}
$$

where $p_{k}$ is the pressure, $\overleftrightarrow{\tau_{k}}$ is the viscous stress tensor, and $\overrightarrow{\mathrm{g}}_{\mathrm{k}}$ is $\mathrm{k}_{\mathrm{the}}$ body force ;

(c) for energy conservation, set

$$
\begin{aligned}
\psi_{k} & =u_{k}+v_{k}^{2} / 2, \vec{J}_{k} \\
& =\vec{q}_{k}-\overleftrightarrow{\mathrm{T}}_{\mathrm{k}} \cdot \mathrm{v}_{\mathrm{k}}, \phi_{\mathrm{k}}=\overrightarrow{\mathrm{q}}_{\mathrm{k}} \overrightarrow{\mathrm{v}}_{\mathrm{k}}+\mathrm{Q}_{\mathrm{k}} / \rho_{\mathrm{k}}
\end{aligned}
$$

where $u_{k}$ is the internal energy, $q_{k}$ the heat flux, ${ }^{\mathrm{Q}_{\mathrm{k}}}$ the internal (reaction) heat source.

It is then necessary to provide \&onstitutive equations of state relating $u_{k}, p_{k}$, $\tau_{\mathrm{k}}, \mathrm{q}_{\mathrm{k}}, \mathrm{Q}_{\mathrm{k}}$ to the mechanical variables $\mathrm{p}_{\mathrm{k}}, \overrightarrow{\mathrm{v}}_{\mathrm{k}}$, the temperature $T_{k}$, and entropy $s_{k} \cdot$

Finally, boundary conditions need to be provided, specifying the inter-relations between the phases. These must be carefully formulated (Ishii 1975), and their form depends on the situation considered. This is another area where significant contributions can be made. No completely satisfactory formulation exists which includes reactive systems. The type of considerations necessary for this difficult, but potentially rewarding, undertaking are very well suited to physicists' specialized skills. It is important to note that this requires judicious and careful modeling to retain the essential physical phenomena and still deal realistically with the varied situations which arise.

Descriptions dealing with dispersed flow beyond this instantaneous local level require averaging of the equations, either spatially, temporally, or using statistical averages (Ishii 1975). The type of averaging used depends on the nature of the problem and leads 
to at least two versions of averaged equations of motion: a two-fluid type description with averaged properties, or a diffusion type model. Physical interpretation of the resultant averaged terms and association of correct kinematic coefficients such as viscosity, mobility, diffusion lengths, and heat conductivity, with these terms is a substantial undertaking. Several such formulations for non-reactive flows (Drew and Segel, 1971; Slattery, 1972, Delhaye, 1973), and reactive flows (Squires, 1979; Davidson and Keairns, 1979; Grace, 1971; Wen and Fan, 1975) exist.

Modeling specific situations using these averaged equations remains an unresolved challenge, even without including reactions. No clear rules exist for choosing appropriate lengths or times for averaging, and scaling laws would be helpful in delineating the various regimes. Separation of short time, small scale turbulent effects from the long-range effects appears to be necessary, but procedures for doing this are not available.

The complexity of the problem has led to the development of one-, two- and three-dimensional computer programs for modeling coal utilization processes. The one-dimensional models involve an average over the cross section of the device; the two-dimensional ones assume a symmetry axis. The programs utilize -- at a minimum -- time averaging of the turbulent motion, and are almost entirely of the Eulerian type. For examples of such programs the reader is referred to the literature (Launder and Spalding, 1972; Patankar and Spalding, 1973; Denn, Yu and Wei, 1979). These programs include combustion reactions in the form of time-dependent global reactions. Some of the faster reactions are treated as being in equilibrium.

It is in general not feasible to compute all details of fluid motion and follow all reactions in three dimensions, even on today's large computers. In addition, the input data necessary for this much detail do not exist. The equations are invariably "stiff" with time scales varying over many orders of magnitude. Thus, a substantial amount of modeling must be done in structuring these computer programs. Here again it is apparent that idealizations, of the type that physicists' backgrounds lend themselves readily to, are important. Those models which are based on the dominant physical phenomena, and are not cluttered with details which are present but do not add significantly to our understanding, will point the way to measurement of a select set of input data. Exciting possibilities for the use of computer "experiments" in producing significant advances in both non-reactive and reactive flow theory are present in this line of research.

Another type of computer program which utilizes a combined Langrangian and Eulerian approach, has been used in modeling combustion engines (Butler et al., 1980). This technique could also prove useful in coal combustion.

It is clear from the above discussion that there are many research areas in which substantial contributions can be made in coal utilization modeling. The approach and type of idealization which form physicists' training seem specially appropriate in this area.

\section{Need for model test facility}

The above brief survey of modeling aspects of coal utilization serves to show that it is not possible at this time to reliably model any coal utilization system starting from first principles. In fact, modeling of existing working systems is only possible when the operating flow regime is know from observation, if then. Much research is needed in establishing the validity and limitations of the various concepts used in the idealization of both non-reactive and reactive flows.

Under current procedure, instrumentation efforts are devoted almost entirely to operation and control of working systems (see Section IV B). Even for prototype models of new systems, the instrumentation is goal oriented, aimed primarily at producing a particular result associated with the process being considered. Systematic collection of data on the reliability of any modeling concept is at best a by-product of such research efforts.

It is apparent that an experimental facility whose primary goal is data acquisition for the testing of models and modeling concepts would contribute substantially to progress in our understanding of coal utilization processes. Such a facility needs to be designed to permit testing of a large variety of flow regimes and conditions. The instrumentation needs to be especially carefully designed to yield information directly pertinent to concept and 
model validity and limitations. This could be accomplished with currently existing techniques normally used in development of prototype instruments.

Such a model test facility would not only yield information useful in coal utilization, but would also provide data important to progress in fundamental research in the physics of turbulence. It should be recognized that this cannot be accomplished as a by-product of prototype process development, because of the goal-oriented "boundary conditions" which are implicit in industrial and government sponsored process research. There is also the question of proprietary rights which may interfere with dissemination of data pertinent to this research area, and its critical analysis by a community of peers.

\section{Theoretical aspects: Summary and}

\section{recommendations}

1) We recommend the establishment of an experimental test facility, appropriately instrumented, dedicated to research on theoretical modeling concepts. Validation of models for the various flow regimes, and establishment of the limitations or concepts used in the construction of models, are sorely needed areas of research. There exists no mechanism currently for funding of such research on a systematic basis. Such a facility would provide information fundamental to progress in the physics of turbulent multi-phase flow, which would also have impact on the understanding of coal utilization processes.

2) Combustion research appears to have special institutional barriers to information exchange because it is an established, commercial ongoing effort, with heavy reliance on empirical data for proprietary configurations. At present, in the "combustion community" there is no obvious mechanism for providing feedback between experimental research, theoretical modeling, and system operations. (For gasification and liquefaction, pilot plant design research efforts, laboratory research, etc. provide natural channels for this communication.) Because we view theoretical modeling as being highly coupled to both fundamental experimental research and to plant operation and design, we recommend setting up a mechanism, such as regular workshops or meetings, e.g. under EPRI sponsorship, for the communication of needs and information between workers in theoretical, experimental, and process design and operation.

3) For both gasification and combustion reactors, current models appear to handle adequately some, perhaps even most, gross aspects of the reactors such as overall efficiency and major chemical output constituents. However, new and more stringent requirements concerning NOX, SOX and POX (small particulate) production require greater understanding of process details and spatial inhomogenities, hence refinement of current models to include some greater detail is necessary. There are in existence models which have enormous detail compared to the simpler models referred to above. However, it is questionable whether enough reliable theoretical or experimental input data exist to make these models viable. There is a need for carefully constructed additions to the simpler models, tailored and experimentally verified, capable of relating these minority constituent outputs to operating conditions. It is envisaged that small changes in operating conditions could be effected which would not reduce operating efficiency while affecting these unwanted outputs substantially.

4) Further progress in the theory of single-phase turbulent flow would benefit our understanding of both combustors and gasifiers. Many combustion reactors rely on turbulence as an active transport mechanism, but even the steady state gasifier conditions must deal with turbulent flow. This is particularly important in entrained bed and in fluidized bed gasifiers. The mathematical models for gasifiers include turbulence only in terms of transfer parameters, such as the Reynolds number scaling parameters; for combustion, semiempirical formulae are used primarily. The proper theoretical treatment of turbulence is poorly developed. A theoretically sound approach to modification of the Navier-Stokes equations to include turbulence in a fashion appropriate to these situations would be very welcome. Such efforts should be vigorously pursued, simultaneously with seeking further advances in semi-empirical models such as those of Patanker and Spalding (1973).

5) Another area in which theoretical development would be extremely useful is multi-phase flow. The problem of minimizing energy consumption in water-coal slurry 
transport is an example of two-phase flow; inclusion of particle size distributions and stability of suspension are but two examples of problems of current interest in the realm of fluid mechanics. Treating reactive, turbulent two-phase flow is much more difficult. In both the multiphase flow area, and the single-phase flow area of the previous paragraph, both theoretical formulation and further computer modeling are needed.

A central problem in multi-phase flow is the establishment of a "flow regime", i.e., a steady or temporal spatial configuration of the phases of the flow; given the flow regime it is often possible to perform some analytic modeling. In recent years, "averaging theory" has been used to account for interphase transfer effects. It may be possible in certain cases to model some multi-phase aspects by Monte-Carlo techniques, as they suggest a means of decoupling from the initial conditions, based upon the flow regime.

6) Only a little theoretical work has been done associated with direct liquefaction, because of the many unknown aspects of that problem. However, it may be possible to use limited and qualitative models to aid in the interpretation of experiments in pilot plants or separate experimental simulations.

\section{References}

Anthony, D. B. and J. B. Howard, 1976, AIChE J. 22, 625.

Arthur, J. R., 1951, Trans Farad. Soc. 47, 164.

Beer, J. M., 1980, 18th Symposium (International) on Combustion, The Combustion Institute, p. 149.

Blair, D. W., J. O. L. Wendt and W. Bartok, 1977, 16th Symposium (International) on Combustion, The Combustion Institute, $\mathrm{p}$. 475.

Butler, T. D., L. D. Cloutman, J. K. Dukowicz and R. B. Krieger, 1980, in Combustion Modeling in Reciprocating Engines, edited by J. N. Mattavi and C. A. Amann, Plenum Publ., New York, p. 231.

Davidson, J. R. and D. L. Keairns, editors, 1979, "Fluidization," proceedings of the 2nd Engineering Foundation Conference, published by Cambridge University Press.

Delhaye, J. M., 1973, In. Jour. Multiphase Flow 1, 395.

Denn, M. M., W-C Yu and J. Wei, 1979, Ind. Eng. Chem. Fundm., 18, 286.
Drew, D. A. and L. A. Segel, 1971, Studies in Appl. Math 50, 205.

Gershman, R., M. P. Heap and T. J. Tyson, 1977, Proc. Second Stationary Source Combustion Symp. Vol. V, p. 65 EPA Publication EPA-60017-77-073e.

Grace, J. R., 1971, AlChE Symp. Series, No. 116, Vol. 67, 159-167.

Gunnarson, O., H. Hjelmberg and B. Lundquist, 1976, Phys. Rev. Lett. 37, 292; Surface Sci. 63, 348, 1977; Surface Sci. 68, 158, 1977 .

Hardesty, D. R., 1980, private communication.

Ishii, M., 1975, Thermo-fluid Dynamic Theory of Two-Phase Flow, Eyrolles, Paris, France.

Kosky, P. G., and J. K. Floess, 1980, Ind. Eng. Chem. Proc. Des. Dev. 10, 586.

Lamb, W. J. Jr., 1980, unpublished.

Lang, N. D., and A. R. Williams, 1978, Phys. Rev. B 18, 616.

Launder, B. E. and D. B. Spalding, 1972, Mathematical Model of Turbulence, Academic Press, NY.

Levy, J. M., L. K. Chan, A. F. Sarofim, and J. M. Beer, 1980, 18th Symposium (International) on Combustion, The Combustion Institute, p.....

Longwell, J. P. and M. A. Weiss, 1955, Ind. Eng. Chem. 47, 1634.

Lowe, A., T. F. Wall and I. McStewart, 1977, AiChE 23, 440.

McKee, D. W., 1974, Carbon, 12, 453.

Mellor, A. M., 1972, in Emissions from Continuous Combustion Systems, W. Cornelius and W. G. Agnew, eds., Plenum, NY.

Mulcahy, M. F. R., 1977, Conference presentation (unpublished).

Mulcahy, M. F. R. and I. W. Smith, 1969, Rev. Pure and Appl. Chem. 19, 81.

Neville, M., R. J. Quann, B. S. Haynes, and A. F. Sarofim, 1980, 18th Symposium (International) on Combustion, The Combustion Institute, p....

Patankar, S. V. and D. B. Spalding, 1973, $14 \mathrm{th}$ Symposium (International) on Combustion, The Combustion Institute, $p$. 605.

Poppe, D., 1980, Chem. Phys. 45, 371.

Reinhart, W. P. and J. Jaffe, 1981, in Quantum Mechanics and Mathematics in Physics and Chemistry, Plenum Press, NY.

Sarofim, A. F. and J. M. Beer, 1978, 17th Symposium (International) on Combustion, The Combustion Institute, p. 189. 
Slattery, J. C., 1972, Momentum, Energy, and Mass Transfer in Continua, McGraw-Hill.

Smith, J. R., 1980, "Theory of Chemisorption," edited by J. R. Smith, Topics in Current Physics, Vol. 19, Springer-Verlag, NY.

Solomon, P. R., 1980, Proc. of Conf. on the Chemistry and Physics of Coal Utilization, West Virginia Univ., Morgantown, W.Va. (to be published).

Song, Y. H., J. H. Pohl, J. M. Beer and A. F. Sarofim, 1980, private communication (unpublished).

Squires, A. M., 1979, "Applications of Fluidization in Coal Technology," Research Notes, published by Virginia Polytechnic Institute and State University.
Ubhayakar, S. A. and F. A. Williams, 1976, J. Electrochem. Soc. 123, 747.

Wen, C. Y. and L. T. Fan, 1975, Models for Flow Systems and Chemical Reactors, Marcel Dekker, NY.

Wendt, J. O. L., 1980, Prog. Energy Combust. Sci. 6, 201.

Wendt, J. O. L., D. W. Pershing, J. W. Lee and J. W. Glass, 1978, 17th Symposium (International) on Combustion, The Combustion Institute, p. 77.

Williams, F. A., 1965, Combustion Theory, Addison-Wesley, Reading.

Woodal1-Duckham, Ltd., 1974, "Trials of American Coals in a Lurgi Gasifier at Westfield, Scotland," NTIS FE-105.

Yoon, H., J. Wei and M. M. Denn, 1978, AlChE J. 24, 885. 


\section{D. MATERIALS IN COAL CONVERSION AND UTILIZATION}

\section{Introduction}

The past three decades have seen great strides in materials science. Physics and physicists - especially solid state physicists - - have played an important role in this development, and a significant fraction of all physicists now work in materials related areas (COSMAT, 1975). Materials science, it is well known, is a multidisciplinary enterprise and one which requires the closest cooperation between engineers and scientists with a wide range of specializations and interests.

Despite the progress realized, it seems fair to say that the advance of modern technology is more often limited by the properties of available materials than by any other single factor. This is especially true of energy conversion technologies in general and of coal technology in particular. Of course the materials constraints are often further complicated by economic ones. Stainless steel, because it is more expensive and more difficult to fabricate than common steel, may not be used in every case where it performs better. Solutions to materials problems must be economically feasible as well as scientifically elegant.

Many of the materials problems that arise in coal technology are familiar in other contexts (e.g. hydrogen attack, corrosion, erosion, etc.), but they appear in coal technology in particularly severe form or as interacting effects. Because of coal's omnipresent hetero-atom and mineral content, for example, the technology must contend with corrosion by multiple oxidants or with simultaneous corrosion and erosion, etc. Other problems arise which are unique to coal technology; in MHD generation, for example, the electrical transport mechanisms and properties of slags become important.

Both the general materials problems and those peculiar to coal technology are well recognized by the DOE and other federal agencies. The federal government has done much to encourage basic and applied research in almost every important aspect of materials science. Still, much remains to be done, and new scientific developments continually open new pathways to improved materials science. This section is devoted to discussion of a few urgent materials problems and of their context in coal technology.

The selected topics are: environmentally promoted

fatigue and fracture

erosion

corrosion

properties of structural

ceramics

properties of refractories and slags

These topics may be recognized as involving to a great degree the chemical physics of surfaces and interfaces, together with diffusion and microstructure. We believe that recently discovered or applied scientific techniques should facilitate much accelerated progress in the study of such phenomena. The topics are taken up in turn and in each case provided with a brief discussion of the context in coal technology.

\section{Environmental fatigue and fracture}

\section{a. The hydrogen environment}

Coal gasifiers operate at high temperature, and one of their most important products is hydrogen; liquefiers operate at moderately elevated temperature and high pressure, and one of the principal reactants fed to them is hydrogen. (See Section IV-A 5 , 6) In each case the effects of hydrogen attack and hydrogen embrittlement upon reactors and containment vessels must be reckoned with. Coal and its products, moreover, generally contain alkali salts and other impurities of such a nature that stress corrosion cracking is a common problem. "Hydrogen attack" and "hydrogen embrittement" denote different phenomena, neither of which is fully understood.

The deterioration of materials in the presence of a hydrogen environment can take place in two general ways: by chemical attack on one or more phases within the material, and by a physical embrittlement of the lattice. Both lead to embrittlement of a material and, ultimately perhaps, catastropic crack growth. The first, called "hydrogen attack", can take the form of a methane bubble formation from the reaction of hydrogen with the carbon in the steel, or it may be metal hydride formation. The purely physical attack known as "hydrogen embrittlement" appears to arise because interstitial hydrogen may disrupt the lattice structure. The effect is strong 
enough so that a material may fail rapidly.

The kinetics and finer details of these processes are not clearly understood. For example, recent work on crack initiation and propagation under hydrogen attack conditions have indicated that plasticity is enhanced (flow stress reduced) prior to ultimate embrittlement (Tien et al, 1980).

In coping with problems of hydrogen attack the chief design tool is the Nelson curve (Nelson, 1969), which shows the conditions of vessel temperature and hydrogen partial pressure below which the steel will not fail by hydrogen attack. Such failures from formation of high pressure methane bubbles by reaction with the carbides concentrated at the grain boundaries are used empirically to develop the Nelson curves from operative experience. However, they should be derivable theoretically from the criterion of the lowest temperature (at a given hydrogen pressure) for decarburization or methane bubble formation from hydrogen reacting with grain boundary carbides. As yet this has not been done.

"Hydrogen embrittlement" signifies the hydrogen induced reduction of ambient temperature ductility of materials such as iron and steel through what appears to be a mechanism more intrinsic than those of hydrogen attack. One theoretical interpretation of this effect involves the use of pair potentials both to analyze an iron lattice containing interstitial hydrogen atoms and to model atomic interactions at a crack tip in such a lattice (Wilson, 1980; Gehlen et al, 1972). Stress required to cleave the hydrogen impregnated lattice is found to be much lower than that for plain iron. More work of this nature may improve our understanding of hydrogen and other lattice embrittlement phenomena such as stress corrosion cracking.

\section{b. Crack nucleation and growth}

Physics researchers are beginning to address some of the basic questions concerning both static and dynamic crack characteristics. The basic approach, in contrast to the classical linear elasticity continuum mechanics treatment, is to investigate the influence of the discrete atomic nature of a solid on the energetics and kinetics of crack propagation, including the highly non-linear interations at the crack tip. The major approach so far has been theoretical with the models varying from analytical treatment of simple models (Fuller, 1978) to rather elaborate computer simulations (Paskin et al, 1980; Wilson, 1980), with emphasis on the response of brittle solids. Several important points have been emphasized: 1) the intricate interplay between crack propagation and dislocation generation (Paskin et al, 1980; Rice and Thomson, 1974), 2) the limitations of the Griffith continuum theory (Fuller and Thomson, 1978; Paskin et al, 1980), 3) lattice trapping and thermally activated crack growth (Fuller and Thomson, 1978), and 4) the interaction of a crack with an ambient atmosphere (Wilson, 1980; Thomson, 1980), e.g., hydrogen embrittlement. In connection with item 4) two avenues have been opened up: a) application of general rate process theory and thermodynamics, and b) computer simulations in which the system is built up from a quantum mechanically controlled local metal-hydrogen reaction through the atomic response of the metal matrix (via pair potentials) to the large scale continuum response (Gehlen et al, 1972; Wilson, 1980). The emphasis has been on the environmental weakening of the bonds at the crack tip. However, the possibility of a chemical bridging reaction increasing the toughness has also been suggested. This point is well worth emphasizing. There is no a priori reason why the environment should only act to weaken the bonds at the crack tip. Studies are needed to find environmental conditions which might actually strengthen the crack tip, and thereby prevent further cracking. The theories, although very preliminary, are highly suggestive and should be pursued actively.

Much less is known theoretically about crack nucleation, which is presumably controlled by bulk and surface inhomogeneities primarily (e.g., nucleation and growth of cavities at grain boundaries and coalescence into cracks, nucleation and growth of methane bubbles, dislocation interaction with precipitates, etc.). Fracture control of structures, based on fracture mechanics (Lawn and Wilshaw, 1975; Wells, 1981), utilizes probabilistic mechanical analysis, fatigue life analyses, or other forms of stress analyses based on the operation for which the structure is intended, combined with knowledge of the properties of the material of which the structure is made. One of the key design parameters is $\mathrm{K}_{1 \mathrm{C}}$, the fracture toughness, which is a measure of the failure stress for a 
given crack size. The fracture toughness for unstable crack growth and failure is often reduced by environments which promote crack growth. Thus the crucial step influencing probable failure in service is subcritical crack growth to the critical crack size (Tomkins, 1981). Frequently the stress driving the crack is of a transient nature involving thermal stresses during startup and shutdown, supplemented by residual and operational stresses. Thus, the subcritical crack growth problem has a fatigue nature in which the rate of crack growth is a function of the stress cycle and the stress.

The base line for fatigue crack growth is a vacuum environment in which minimum crack growth is observed. There is considerable effort on the phenomenological aspects of subcritical growth; and, as indicated above, investigation of the pertinent atomic interactions are beginning. Little is known of the mechanisms for the acceleration of the crack growth in aggressive environments, such as hydrogen or acid chlorides. It is here that physics might have an even more significant contribution to make in understanding how the lattice responds to such environments.

\section{Erosion}

In its every facet, coal technology requires the direction and control of fluid streams laden with solid particles. Erosion -- often complicated by corrosion -- is an ever present problem. Coal itself is a conglomerate of solids that display a wide range of mechanical properties. The immediate residue of pyrolysis is char, also a solid. Combustion, gasification, or lique faction invariably leaves a residue of solid ash or mineral matter. Some, at least, of this residue is in a finely divided state and is entrained in the product gases or liquids. Slurry pumps are needed to inject coal-oil or coal-water slurries into reaction chambers. Letdown valves are needed to return the reaction products to ambient conditions. Fans or blowers must move particle laden stack gases through bag-houses and scrubbers. Burner nozzles must handle streams of air with entrained pulverized coal. And, if combined cycle power generation with gas turbines is to become a reality, systems must be designed to cope with ash particles entrained in combustion products and synthesis gas. By itself the mineral matter present in coal is extremely erosive; when combined with the corrosive substances in the process stream (e.g. sulfur, alkali metals, chlorides, etc.), it is destructive to practically all materials.

Solid particle erosion is the mechanical removal of metal (or ceramic) as a result of the impact of solid particles, usually entrained in a high velocity gas or liquid stream. Some specific examples of erosion are: erosion of letdown valves in coal liquefaction systems by ash, mineral, and coal particles suspended in the coal liquids; erosion of gas turbine blades and vanes by solid particles entrained in the hot combustion gases; and erosion of fire side heat exchange tubes in boilers. Erosion is only one of the forms of wear; valve faces may be scored by solid particles trapped between the operating surfaces, for example.

There has been a reasonable amount of basic investigation of erosion processes [see Finnie (1979) and Hutchings (1979)] which has shown that for ductile materials erosion is at a maximum at an impact angle (angle with respect to tangent) of approximately 30 , and that the erosion damage varies with the impact velocity raised to a power greater than 2 . Complementary approaches have analyzed the fluid dynamic determinants of particle impact parameters as carefully as possible, using a largely empirical approach to predict the location and extent of erosion damage. It is customary to study and measure erosion at high wear rates and thus to characterize the erosion resistance of materials. We believe greater emphasis should be placed upon studying threshold effects in erosion (e.g. velocity thresholds, particle size thresholds, etc.). An understanding of thresholds for erosion could contribute importantly to the design of viable systems.

The effect of temperature on erosion is not understood. At elevated temperature, most materials oxidize, so that in many cases the incident particles are eroding the oxide rather than the metals. The characteristics of erosion differ for brittle and ductile materials, and the problem of the erosion of a composite consisting of a hard brittle thin layer on a ductile substrate has received little study. The work of Pettit et al. (1980) has shown that at elevated temperatures, there may be a significant interaction between erosion and corrosion; and qualitative analysis suggests that the effects could be synergistic or antagonistic.

There exists at present only imperfect 
understanding of the mechanism of metal removal during erosion; much more work needs to be done. Erosion mechanisms in more complex systems (two phase alloys, thin brittle layers on ductile metal substrates, coatings, etc.) have not been studied in any physical detail. The nature of the eroding particles varies from coal to coal, and it would be of value to understand how the erosiveness of particulates is related to their other properties (e.g. hardness, strength, and shape). Existing analytical approaches are far from being predictive; they usually do not include the particle properties explicity and are seldom formulated in terms of the properties of the material being eroded.

In short, the fundamental physics of erosion processes is poorly understood and the field appears wide open for theoretical and experimental studies.

\section{Corrosion}

The use of coal in energy systems introduces several problems of corrosion and erosion of the materials of construction. The harsh chemical environment is provided both by the nature of the combustion gases of the coal used and by the presence, depending on process temperature, of liquid coal slag or solid ash particulates. The most severe processes in this regard are those in magnetohydrodynamic generators and the slagging gasifiers. All processes using coal face corrosion and erosion peculiar to, and accelerated by, the special nature of coal as a fuel source containing mineral matter. The subject of coal slag is treated in section IV D, 6 .

Some problems are peculiar to the various energy generation processes. These processes include those in the pulverized coal boiler, the fluidized bed combustors, and turbines in the high temperature combined cycle gasification process.

\section{Pulverized coal boiler}

The pulverized coal (P.C.) boiler is today the mainstay of the power industry, producing almost half the thermal energy for generation of electricity. As the nation converts from oil and gas to coal, the relative importance of the pulverized coal boiler will become even greater.

Analyses of power plant outages (Edison
Electric Institute, 1975, 1976), show that the boiler is the least reliable component of the entire system, followed by turbines, condensers, and generators in that order. The shortcomings of boilers are chiefly the result of corrosion on either the fire side or the steam side of the water walls, superheaters and reheaters. We have only approximate understanding of the corrosion mechanism involved. Corrosion on the steam side perhaps is the easier problem to analyze from a scientific point of view, as variations in coal and firing conditions in various parts of the boiler result in a wide variation in fire side corrosion phenomena. Erosion also is a matter of concern in the combustion chamber, but is less important here than in other aspects of coal technology, such as in coal gasifiers and liquefiers.

A serious problem in boilers that adversely affects the operation of the high pressure and intermediate pressure turbines is the formation of magnetite $\left(\mathrm{Fe}_{3} \mathrm{O}_{4}\right)$ scale on the inner walls of superheaters and reheaters. The scale spalls during temperature cycles and is swept into the turbine, causing solid particle erosion. Only rudimentary information is available on magnetite scale and the stresses generated during thermal cycles that cause exfoliation to occur (Rehn, 1981).

\section{Fluidized bed combustion}

The product of desulfurization in fluidized bed combustion is $\mathrm{CaSO}_{4}$, which is solid but corrosive at the reaction temperature. This in-bed corrosion problem appeared to result from the low oxygen content in the fluidized bed (Stringer and Erlich, 1976). Although the overall oxygen content is about $10^{-1}$ atmospheres (10\% excess oxygen), the oxygen content of the bed itself is very low, about $10^{-12}$ atmospheres, with most of the oxygen contained in the bubbles of air rising through the bed. The equilibrium between so and $\mathrm{O}_{2}$ in the $\mathrm{CaO}_{-} \mathrm{CaSO}_{4}$ system is such that the sulphur potential (fugacity) is about $10^{-6}$ atmospheres. At this sulphur potential, sulfidation of heat exchangers always becomes a matter of concern for metal temperatures from $650 \mathrm{C}$ to the bed temperature of $850-900 \mathrm{C}$. Nickel-based alloys are particularly susceptible because of the low melting point of the Ni-NiS eutectic. Surface melting of the eutectic leads to catastrophic corrosion. Iron-based alloys survive longer. By 
contrast, nickel was a strong performer in the gasification environment (Perkins, 1979). This corrosion problem in the fluidized bed boiler is described below under high temperature corrosion.

The alkali salts in the combustion products from a pressurized fluidized bed may also be sufficient to promote hot corrosion of the expander turbine, otherwise the in-bed heat exchanger corrosion problem in the pressurized fluidized bed combustion is much the same as in the atmospheric fluidized bed combustor.

\section{Turbines}

There are many materials problems in steam turbines, but they are not uniquely associated with coal as the source of heat. Thus, steam turbines and condensers per se will not be covered in this report. The use of coal or coal derived fuels in gas turbines, in the simple Brayton cycle, or in the combined Brayton-Rankine cycle, however, brings characteristic problems of its own and will be discussed.

Use of the high temperature gasification combined cycle is necessary in order to justify the added expense of gasifying coal before combustion, in comparison to burning coal in the conventional pulverized coal power plant equipped with a scrubber. The efficiency of energy conversion in a gasification combined cycle may approach $45 \%$ given the development of suitable advanced gas turbines; whereas a pulverized coal power plant with a scrubber rarely has a thermal efficiency in excess of $35 \%$. The thermal efficiency of the combined cycle plant depends strongly on the combustion temperature in the gas turbine. At least $1200 \mathrm{C}$ is necessary (and $1400 \mathrm{C}$ is desirable) for the inlet temperature to the turbine. The exhaust temperature from the gas turbine should be high enough to raise $540 \mathrm{C}$ steam in a waste heat boiler. There are two known approaches to the high temperature gas turbine for operation in this range - - an uncooled ceramic turbine or an air-cooled or water-cooled metal turbine.

Metallic turbines used in combined cycle plants using coal-derived fuels will be exposed to corrosive agents and particulate matter that might adversely affect life expectancy. The chief agents of corrosion are alkali metals which combine with residual sulfur in the combustion products to produce a condensed salt of sodium or potassium sulfate on the metallic turbine section. In the temperature range above its melting point and below its dew point, the condensed salt can cause accelerated corrosion. The coal derived fuel must therefore be purified sufficiently to permit the metallic turbine alloys to operate without excessive corrosion.

\section{Low temperature corrosion}

Coal conversion systems also present a number of situations where low temperature corrosion may be encountered. The acid corrosion due to high concentration of sulfur oxides, or the fractionation of impurities to aqueous condensates which contain relatively high concentrations of dissolved salt, can initiate corrosion on surfaces. These in turn, act as stress raisers and can initiate a fatigue failure. Corrosion may also occur from the water soluble components of the coal (i.e. slurries) or from the presence of chlorides, organic acids, water, or sulfur compounds in non-aqueous liquids.

No distinction has been made here between the various modes of corrosion which may include: broad front attack, crevice corrosion, pitting corrosion, or the possible interactions between corrosion and mechanical effects such as: corrosion fatigue, fretting corrosion, stress corrosion-cracking. In fact, stress corrosion-cracking is likely to be a particular problem because of the presence of chlorides in coal. The role of the physicist here would be to look for systematic behavior in the growth phenomenology of these corrosion modes as an application of the general problem of the interaction between solid surfaces and the gaseous or liquid environment in contact.

\section{Mixed oxidant attack and molten salt corrosion}

Most of the hot corrosion problems can be generalized into 1) mixed oxidant attack and 2) molten salt corrosion. In gasifiers, in some regions of fluidized bed combustion systems, in substoichiometric ("fuel-rich") regions of low NO staged combustors, and in some other special situations, envirgnments exist with low oxygen potentials $\left(10^{-12}-10^{-24}\right.$ atm) and relatively high sulfur $\left(10^{-4}-10^{-7}\right.$ atm) and carbon (0.1-1.0 atm) activities. In these environments mixed oxidant attack is a problem (Stringer and Erlich, 1976). 
The other major form of high temperature corrosion involves the presence of molten salts, usually of the alkali metals, on the metal surface. The gas phase is usually the combustion product gas, and is typically relatively oxidizing with oxygen potentials in the range 0.01 to $5 \mathrm{~atm}$. This salt-induced attack is often called hot corrosion, and has been a major problem in oil-fired gas turbines for many years. A similar problem is encountered in the radiant superheater of coal-fired boilers.

\section{Mixed oxidant attack}

Mixed oxidant attack is caused by the transport of one of the minor oxidants (sulfur or carbon) through the (otherwise "passivating") oxide layer on the alloy surface. The minor oxidant then reacts with the metallic element responsible for forming the protective oxide (usually chromium or aluminum) to form a dispersion of sulfide or carbide particles within "the depleted oxide matrix." If these particles are coarse enough, they then oxidize in situ as the local oxygen activity increases. This prevents the maintenance of the protective oxide. Often, the sulfur or carbon released by the oxidation of the sulfide or carbide does not escape back into the atmosphere, but makes its way further into the metal, so that the process, once initiated, may be self-maintaining. It is thought probable that the transport of the minor oxidants through the external oxide layer takes place through low resistance paths, such as grain boundaries, dislocation cores, pores, or filaments of a second phase. There has been a a considerable volume of work on the phenomenology of mixed oxidant attack, principally aimed at the problem of materials in gasification systems (Hill et al, 1979; Perkins, 1979). Analysis of gasifer corrosion by Perkins reveals evidence of cation motion through the oxide as the principal mechanism of sulfidation. Stringer (1977) shows evidence that in conventional power plant corrosion, anion motion is dominant. Clearly some very fundamental studies are necessary to clarify this problem.

\section{Molten salt corrosion}

Molten salt induced hot corrosion in gas turbines has been the subject of much study. (See the recent review article of Stringer, 1977). The boiler superheater problem is discussed at length in the volume by Reid (1971). Essentially, there are two aspects to the reaction. The simplest situation is when the deposit is a molten layer of sodium sulfate. This may act as a diffusion barrier to oxygen so that at the metal/salt interface there is a low oxygen activity. The consequent dissociation of the sulfate results in a high sulfur activity, leading to sulfur attack.

The other aspect is that the molten salt may be capable of dissolving ("fluxing") the protective oxide layer. Usually, protective oxides are stable in the neutral (stoichiometric) alkali sulfate, but cease to be stable if the salt becomes sufficiently basic (alkali-rich) or acid ( $\mathrm{SO}_{3}-\mathrm{rich}$ ). The acidity of the salt will be a function of the alkali content and the local $\mathrm{SO}_{3}$ partial pressure in the environment. For transport rates to be rapid enough to cause serious attack, the salt layer must be molten, so constituents that can generate a low-melting point phase will consequently increae the extent of observed attack. For this reason sodium sulfate induced corrosion is observed at temperatures as low as $575 \mathrm{C}$ even though the anhydrous sulfate melts at $884 \mathrm{C}$.

\section{Research issues of interest to physicists}

Because little generalization has been possible with the-wide diversity of high temperature corrosion problems, it has been difficult to sift through the details in order to get a clear picture of research needs. But in most environments of concern in energy systems, virtually all metals of construction are unstable with respect to their oxides, sulfides, carbides and so forth. An oxidation-resistant alloy owes its resistance to the formation of a chemically and mechanically stable protective oxide layer on its surface. Further oxidation then involves the transport of one or more of the reactants through the oxide, and if the transport rates are sufficently slow, the reaction rate is low enough so that in effect the alloy does not degrade appreciably in times comparable with the design lifetime of the component. In practice, this means that the mechanical adhesion of the as-grown oxide to the substrate must be good. For high-temperature oxidation resistance, the protective oxides are usually $\mathrm{Al}_{2} \mathrm{O}_{3}, \mathrm{Cr}_{2} \mathrm{O}_{3}$ or in some cases $\mathrm{SiO}_{2}$, 
although other oxldes are theoretically possible.

Development of a protective oxide on an alloy surface involves a number of criteria. Obviously, the protective oxide must be thermodynamically the most stable phase in contact with the alloy. The diffusion of the oxide-forming element ( $\mathrm{Cr}, \mathrm{Al}$, or $\mathrm{Si}$ ) out from the alloy to the oxide/metal interface must be such that a continuous adherent external oxide, rather than an internal distribution of oxide particles, forms. Finally, when the protective oxide is nucleated on the surface, it must spread sufficiently rapidly over the metal surface to form a continuous layer before it is mechanically disrupted by less stable oxides.

Mechanical adhesion of the protective oxide to the substrate is a complex topic involving the familiar aspects of interfacial energies, but also the more qualitative aspect of the development of an irregular re-entrant interface ("keying") and the establishment of a growth process which allows the oxide to follow the retreating alloy surface without the development of voids between the oxide and the substrate.

Multicomponent diffusion theory is basic to a full understanding of alloy oxidation. This subject is still at an early stage. Nucleation of oxide phases, the growth of the nuclei across the surface, and the competition between oxides of differing stability and growth rates are topics of concern. Work is currently being conducted along these lines. A good starting point to look is Whittle (1972) and Smeltzer et al (1976).

Transport processes in oxides, and in particular, the transport of minor species such as sulfur or carbon, are of interest. Short-circuit transport is of at least as much interest as bulk diffusion. The oxide/metal interface is not well understood, and indeed is poorly characterized. Much work needs to be done on the interface in "dynamic systems" in order to understand the mechanisms of mechanical adhesion. The detailed structure of the external oxide layer needs a great deal more work. It is probable that the initial nucleation processes on a metal surface exposed to a gas mixture may have a profound effect on the later course of the reaction. The techniques of surface physics should contribute substantially to a solution of these problems.

Protective oxides may be either those naturally occuring on a material surface, or they may be composed of components foreign to the normal alloy structure. A particularly intriguing subject for future research is expanding the area of modification of surfaces to withstand environmental streses such as sulfidation, stress corrosion cracking, erosion, sub-critical crack growth, molten salt attack, hydrogen embrittlement, etc. In addition to protective oxide formation on the surface of the metal or alloy there are also surface coatings such as chromiding and surface modification via diffusion and ion implantation. There is still much fundamental work to be done in understanding the role of, and developing our ability to modify, the surface chemical and physical structure of a material.

Ion implantation is in its infancy as a technological tool and holds much promise for the future. Progress has already been made in strengthening the wear resistance of bearings by this technique on a commerical scale and some progress on an experimental scale made in strengthening a material against corrosion (Antill et al, 1980; Collins et al, 1980). Both theoretical and experimental surface studies can contribute substantially to the extension of the technology of surface modification by fundamental physical studies of the formation and properties of these surface layers.

One type of corrosive attack at low temperatures that deserves the special attention of physicists, and one that is certainly a concern in advanced coal conversion systems is erosion-corrosion. Erosion-corrosion is a distinct form of localized attack that occurs on some metals in areas where the turbulence at the metal surface is high enough to cause mechanical or electrochemical disruption of a protective oxide or other surface film. It is not simply two effects occuring simultaneously. The process is usually accelerated when abrasive solid particles are entrained in the water, but solid particles are not a prerequisite for erosion-corrosion. Some aspects of erosion-corrosion are understood. For instance, because turbulence increases with increasing velocity, higher water velocities favor the initiation of erosion-corrosion. Flow geometry also influences turbulence intensity, and is as important as velocity in determining whether the protective film will be disrupted.

There is no agreed upon theory of erosion-corrosion. Many believe in the 
"critical shear stress" theory in which the oxide or other surface film is mechanically stripped from those areas on the metal surface where the shear stress exceeds a certain critical value. But it has been pointed out that the shear stresses actually encountered in practice are very small ( $\ll 1$ psi). Others prefer the "electrochemical breakdown" theory, in which the level of turbulence intensity influences the localized electrochemistry at the metal surface, and causes local electrochemical (not mechanical) breakdown of the protective film. There is also no clear picture of the role of solid particles entrained in the water. The size of particles, the volume loading, the particle shape, and the hardness all have an effect on the rate of erosion-corrosion but only empirical relationships have been derived so far. We need to investigate erosion-corrosion in greater depth in an attempt to eliminate controversy, explain the influence of the various important variables, and come up with a successful theory of erosion-corrosion that is applicable to particle-free and particle-loaded waters alike.

\section{Structural ceramics}

Structural ceramics are a class of high strength, high density, brittle materials generally hot pressed and sintered from the powder. The most common materials are the carbides and nitrides of silicon and boron. Many researchers throughout the world are engaged in processing and structure research to find ways of producing desirable microstructures or of fabricating specialized shapes for use in devices like gas turbines.

The first major effort to apply ceramics in gas turbines was started by ARPA/DOD, and is presently continuing with DOE support, mainly for the development of automotive gas turbines, but also for large industrial gas turbines for electricity generation. Early efforts concentrated on developing a design technology for brittle materials and establishing a data base for the most promising ceramics. Although this proved more difficult than anticipated, a systematic and generally accepted design methodology has emerged, a materials data base for short term application has been established, and some ceramic parts have been successfully tested in truck turbines and low pressure heat exchangers (Mangels, 1978; Uy et al, 1978;
Williams and Uy, 1978). Long term performance of ceramic components, however, has not been proven.

The corrosion/erosion resistance and high temperature strength of structural ceramics is considered to be excellent and far superior to that of superalloys. For temperatures below $1100 \mathrm{C}$ this has been fairly well documented (Sims and Palko, 1977; Freimann et al, 1978) but data above $1200 \mathrm{C}$ are very scarce and of ten conflicting. The reason for this is that most structural ceramics contain sintering aids, which may become viscous at elevated temperatures or react with species in the environment. This can lead to accelerated corrosion and slow crack growth which markedly reduce the useful service life of ceramic components. Reliable long term service above $1100 \mathrm{C}$ must be demonstrated, prior to any large scale component development effort. The interaction between subcritical crack growth and the environment particularly needs to be defined.

Performance of structural ceramics is currently limited by subcritical crack growth at elevated temperatures. This can be accelerated by corrosives in the environment. A thorough study of subcritical crack growth as a function of microstructure, grain boundary impurities, temperature, and environment expected in coal combustion or conversion should be made.

Some studies should be done on single crystal materials for reference, especially if ways can be found to grow single crystals of high purity silicon carbide and silicon nitride to determine baseline properties such as subcritical crack growth, creep, erosion and corrosion resistance. If large single crystals having acceptable properties can be grown, single crystal components with superior properties might prove feasible.

Non-destructive examination (NDE) refers to a body of techniques used to search for critically sized flaws in engineering materials (Coffey and Whittle, 1981). These techniques are used to study both the bulk material and the welds used to assemble the final structure. They most commonly include radiography, fluorescence, and ultrasonic probing. Radiography is conventional $\mathrm{X}-\mathrm{radiation}$ photography; fluorescence uses a special coating to expose surface flaws; and ultrasonic scattering can identify the size and location of a flaw. Ultrasonic waves can have wavelengths of a micron or less.

Critical flaw sizes in metallurgical 
materials are cracks generally of the order of $1-3 \mathrm{~cm}$ long, large enough for radiography and fluorescence to be useful. Critical flaws in structural ceramics can be much smaller than those in metals. Thus, present non-destructive evaluation (NDE) techniques cannot be carried over directly from metals to ceramic components. Attempts are under way to develop NDE methods which will detect flaws in the 50-100 $\mu \mathrm{m}$ range for structural ceramics. This work should be reviewed to determine if additional work or a more fundamental approach is needed. Further development of acoustic imaging techniques to identify both the size and type of flow would be useful. A good source of information concerning the application of NDE techniques is the new EPRI NDE Center in Charlotte, North Carolina.

The prevailing design methods for brittle materials are based on statistical techniques which use the Weibull function (Weibull, 1951) to characterize the relation between the initial flaw size distribution and the failure probability. Although this function applies reasonably well in most cases, its validity has not been checked thoroughly, and it is not known whether its application leads to conservative or optimistic design. A fundamental study to validate the physical basis of the present Weibull design methodology for brittle ceramics is desirable. Work on statistical models of failure of ceramics is a noteworthy example of this approach (Evans and Rame, 1979); and probabilistic failure mechanics is a field of study in itself (see Rau and Besuner, 1981 and Section IV-D-2b).

\section{Refractories and slags}

For most applications above $1000 \mathrm{C}$, metals are not practical as structural materials except for the refractory metals (tungsten, tantalum, molybdenum, etc.) which find use in strongly reducing environments. On the basis of cost and durability, often, the best choice is to employ nonload-bearing ceramic refractories to insulate the structural materials from the high temperature corrosive environment, which contains both combustion products and mineral matter. Depending upon temperature, the mineral matter can be in the form of a "dry" abrasive ash or a highly corrosive liquid "slag".

\section{a. Refractories}

Refractories are high temperature materials composed of the oxides of silicon, aluminum, magnesium, calcium, chromium, iron, sodium and potassium. They are generally fabricated by mixing the appropriate materials (raw, calcined, or pre-fired and crushed) with a binder and have compositions which often vary with the source of the raw materials. Except in specialized cases, small impurities are not controlled. The exact composition of a refractory varies with production batch within certain limits set by the manufacturer as a compromise between consistency and economy. The structure tends to be a mixture of crystalline and glassy phases of many different compositions bonded into a structure of varying porosity. Refractories tend to be brittle, and fracture with little or no deformation. At high temperatures they begin to soften and lose strength. Under mechanical loads they will creep at elevated temperatures, so care is taken in designing with refractories.

Commercial development of refractories for extreme environments has been hampered by a lack of fundamental research into and understanding of the high temperature behavior and interactions of these materials. The role which the microstructure of a refractory plays in resisting degradation should be studied using advanced electron microscopy techniques developed within the last decade. The importance of interfacial phenomena as the rate controling mechanisms in the disintegration process need careful study.

Refractory uses can be divided into low and high temperature applications. Low temperature applications are those below slagging temperature (about 1100C) where coal ash is still in the solid form and does not react with the refractory. Degradation mechanisms are mainly gaseous corrosion and erosion by particulates. High temperature applications are generally above 1250C, where coal ash is generally molten and reacts chemically with the refractory liner.

Refractories for low temperature applications such as liners of dry ash gasifiers, process piping and cyclones, can often be modeled after those used in the petrochemical industry where refractory concretes, both dense and insulating types, are the standard materials. An extensive 1 aboratory and field test program has indicated that low cost $50 \% \mathrm{Al}_{2} \mathrm{O}_{3}$ concretes 
(Bakker, 1978a, 1978b), consisting mainly of calcined kaolin grains bonded with calcium aluminate cement, are very suitable materials. They tend to increase in strength during service because of the hydrothermal formation of fibrous calcium aluminum silicates.

Refractories for high temperature applications -- mainly slagging gasifiers and MHD air preheaters - - are in a less satisfactory state. Laboratory tests, (Kennedy, 1979; Townes et al, 1981; Pollina and Smyth, 1981) have indicated that $\mathrm{Mg}(\mathrm{Al}, \mathrm{Cr}) \mathrm{O}_{4}$ spinels should be suitable. However, refractory wear in actual pilot plants has often been disappointing. It is at present not possible to predict behavior in actual service from systematic laboratory tests. The reasons for this remain to be be discovered.

Physics related research and development in this area would benefit both slagging gasifiers and open cycle MHD power generating systems. Combustor temperatures are high enough to ionize the seeded coal combustion products so that the plasma is highly reactive. In most proposed coal-fired open cycle MHD power generation schemes (Pollina and Smyth, 1981), the required plasma temperature is achieved by preheating the combution air in regenerative heaters employing cored refractory bricks as the heat storage medium.

\section{Phase diagrams}

Interactions between slags and refractories are dependent on thermodynamic equilibria, expressed in various phase diagrams. Slags are multicomponent oxide mixtures, so phase diagrams are needed which have perhaps as many as 9 or 10 components. A large number of phase diagrams are available (Watt, 1959; Levin et al, 1964; Muan, 1979; Muan and Osborn, 1965), but often the pertinent information on coal slags is not available. For example, the phase diagram for $\mathrm{K}_{2} \mathrm{O}-\mathrm{Al}_{2} \mathrm{O}_{3}-\mathrm{SiO}_{2}$ is largely incomplete. Such information is badly needed. In the system $\mathrm{Cr}_{2} \mathrm{O}_{3}-\mathrm{Al}_{2} \mathrm{O}_{3}-\mathrm{SiO}{ }_{2}-\mathrm{FeO}$ for various oxygen partial pressures, the effects of $\mathrm{CaO}, \mathrm{MgO}$ and alkali additives to this systems should be studied. The experimental work is difficult since thermodynamic measurements on these materials at these temperatures are a formidable task. Theoretical work is equally difficult since it involves computer modeling the thermodynamics, and generating phase diagrams with as many as nine dimensions. The experimental data is meager, and such work would be a significant, long term project for both theoretician and experimentalist. Some progress is being made. See, for example, Eliezer et al (1978) and Byker et al (1980). Physicists are particularly equipped to handle both these aspects; by developing new measuring systems and by solving multidimensional thermodynamics problems using sophisticated computer techniques.

\section{Effects of microstructure}

Refractories must be able to withstand thermal shock and to resist penetration, dissolution, and erosion by slags. With present refractory technolgy these requirements are incompatible. Slag-resistant refractories have a high density and low porosity, and in consequence they generally have poor thermal shock resistance.

The ability of a material to withstand a severe thermomechanical environment is determined both by its microstructure and by its chemistry. There is a trade off in fabrication and use between the desire to have dense bricks for resistance to corrosive attack and the desire to have porous bricks for resistance to thermal stresses arising from rapid temperature gradients. Fusion cast (poured from the melt) bricks have very high density with almost no open porosity; and the corrosion pathways through pores, surfaces and along grain boundaries are considerably reduced. However, pores are an important part of the microstructure enabling the brick to withstand thermal stresses. Thermal stress cracks always occur in refractories, but will terminate when the cracks propagate into a pore.

Ceramic materials, as a class, are rigid with high moduli of elasticity allowing very little plastic deformation. Cracks forming at points of stress concentration are not easily blunted by yielding of the surrounding material and will tend to propagate in the brittle fracture failure mode.

Microstresses are developed by phase transformations and by differential thermal expansion, and occur over extremely localized volumes within a material. The microcracking that results is localized most commonly along the grain boundaries separating the phases. Once formed, these microcracks serve to 
relieve microstresses generated in the surrounding matrix (Kingery et al, 1976).

The formation of microcracks and macrocracks during thermal cycling produces irreversible expansion of the material, and the grains and crystals surrounding the cracks tend to push each other apart on heating. During cooling the dimensional changes will not be totally reversible, and the thermal stresses may lead to further, irreversible cracking. The result is a gradual increase of void space and bulk volume and a decrease in strength of the bonding matrix. This decrease in strength may ultimately lead to complete disintegration of the material as is sometimes observed. At present, no theoretical models exist that will describe the effects of multiple thermal cycles on a material. The long-term behavior and strength degradation of ceramics must be determined empirically.

\section{Relationship between structure and resistance to environmental stress}

Physicists might make a contribution by computer modeling these materials to determine the influence of structural parameters or composition changes on the thermal cycling response. Possibly by varying the grain size, or the type of bonding, or the porosity, or the expansion characteristics of the grains, we can substantially improve the situation. Computer modeling can help determine the appropriate microstructure which represents the best compromise for the system studied. It should be kept in mind that the results of any study will be sensitive to the boundary conditions and so a set of solutions may be generated, each applicable to a specific system.

However, a complete model must not only locate points of maximum internal stress, but must be able to simulate the introduction, propagation, and arrest of cracks. That there will be some cracking is inevitable. The goal of the modeling would be to find ways to minimize the effect of cracking upon the gross structural properties of the refractory.

\section{Kinetics of dissolution of refractory oxides in}

\section{coal slags}

Studies of the dissolution of refractory oxides in molten slags were carried out in the 1960 's for the steel industry; the systems studied were single-crystal and polycrystalline alumina in soda-lime glass. Similar dissolution studies using single crystal oxides and spinels present in refractories and typical coal slags should be carried out now to get enough baseline data to optimize refractory compositions for coal conversion technology.

The presence of a hostile chemical environment presents a range of problems. There can be granular, intergranular, or grain boundary attack by one or more elements in the seeded coal slag, leading to complete chemical penetration and failure. This chemical penetration may be accelerated by the thermal cycling which can serve to open and close pores so that the corrosive slag has ready access to the interior. New pores (micro-or macrocracks) may also be created and become avenues of penetration. Long duration at elevated temperatures and at too low an oxygen partial pressure may lead to the growth of new phases within a multiphase brick -- with the subsequent crack generation and failure as described above. Finally, vaporization of the more volatile constituents will also lead to mechanical failure especially if they are the stablizing additives to the material (as a loss of calcia in calcia stabilized zirconia). The extensive microcracking which has been observed in a rebonded magnesia chrome material suggests the presence of microstress producing mechanisms. Leonard and Herron (1972) report a permanent volume expansion and structural damage in magnesia-chrome refractories due to temperature or atmospheric cycling. They contend that the primary mechanism is a cyclic reduction and oxidation due to a changing equilibrium oxygen content. The reduction occurs with a loss of oxygen and the collapse of lattice vacancies to form new closed pores.

\section{Physical properties of refractories}

The experimentalist can develop new techniques to measure physical properties of these materials at elevated temperatures. Items needed by designers are thermal expansion, thermal conductivity, specific heat, creep, elastic moduli, and fracture toughness. It would be extremely helpful if some of these properties such as the last three could be monitored in situ. Typically the thermal diffusivity is measured by the laser-flash technique (Bates, 1970), the heat 
capacity guessed on the basis of simple mixing of known oxides, the density calculated from expansion data, and the thermal conductivity calculated from all of the above.

The problem with this approach is two-fold: a) The laser flash technique works best on specimens such as metals, glasses, and dense polycrystalline materials. It does not work on materials with large grains or pores. b) The heat capacity of silicate materials cannot be evaluated reliably by assuming simple mixing since this assumption has been shown to be invalid in some cases (Howald et al, 1979). While the error may be tolerable for engineering design, it cannot be overlooked by those working on phase diagrams. Obviously any discrepancy is of crucial importance to the phase chemists and physicists.

There are few reliable differential scanning calorimeters usable with any accuracy above about $800 \mathrm{C}$; most designs break down in the radiation regime. The few commercially available drop calorimeters are quite expensive; while the construction of an accurate drop calorimeter for high temperature application requires major financial outlays and labor intensive effort using present concepts. It should be investigated whether other thermal techniques using bulk specimens and conventional (ohmic) heat sources in the range 1200-2000C might prove to be useful in measuring thermophysical properties of materials.

Creep is easily measured at high temperature but the elastic moduli and the fracture toughness are not. For ceramic materials which may have many weak bonds or internal cracks, there is a need for stress-strain curves under both tension and compression at elevated temperatures. Few measurements have been done under tension in coarse grained materials such as refractories which have intrinsically low tensile strength. Thermal cycling conditions can produce high tensile stresses in materials.

The goal of any materials testing program is to determine:

Which environmental characteristics

are degrading the material?

What phase of the material

is being attacked?

What is the detailed failure mechanism?

What property or properties in the material and/or environment can be changed to enhance resistance?

\section{b. Slags}

All coals contain mineral matter of from $5 \%$ to $40 \%$ by weight of the coal. This mass of material must be carried through the process and removed for disposal. Some fly ash is used in concrete manufacturing but the ash has little economic value generally. One hopes that such a plentiful and inexpensive substance would find more uses in the future. Although the character, quality, and distribution of minerals may vary considerably within the coal seam, this variation tends to be masked during the mining, transporating, comminuting and other operations which turn the coal into a usable form. Large differences exist in the ash characteristics of coals from different regions. In general, however, it is composed chiefly of compounds of silicon, aluminum, iron, and calcium with smaller amounts of magnesium, sodium, potassium, and titanium. These elements are present as oxides, sulfates, and complex alumino-silicates. Below 1100-1300C the ash occurs as an abrasive solid particle. Above this temperature range the ash is in the form of a liquid "slag" which moves through the system as droplets which coat the walls and lead to fouling (constriction of mass or heat flow). Above the fusion temperature, the liquid ash is highly corrosive and can attack any or all of the phases present in a refractory material. Certain components of the ash, notably some sodium and potassium compounds, are volatile at coal combustor temperatures and will condense on the cooler walls downstream, leading to the hot corrosion discussed earlier.

\section{Slag penetration and wetting of refractories by slags}

The wetting of refractory oxides by various slags is poorly understood. The physical mechanism leading to slag interaction in a porous refractory in the presence of a thermal gradient should also be studied and clarified.

\section{Magnetohydrodynamic (MHD) energy conversion}

The magnetohydrodynamic cycle is a highly efficient energy conversion method, where an electrically conducting high temperature gas is driven through a high magnetic field 
( $6-7 T)$, generating electric power in the process.

The MHD generator is a large combustor which burns coal at $2200 \mathrm{C}$ using preheated air, followed by an electrical generating chamber called a "channel" in which the conducting exhaust gases traverse the magnetic field. To enhance the conductivity of the combustion products, a potassium (seed) compound (such as $\mathrm{K}_{2} \mathrm{CO}_{3}$ ) is added before the plasma passes downstream into the generating channel. The channel is encased in a large superconducting magnet; and two opposing walls are lined with electrodes which transmit the current generated by the induced EMF. The rate of enthalpy extraction for a full scale generator is high enough for the channel plasma to be in a non-equilibrium state.

In the most general case, the path for charge conduction in an MHD channel includes metallic or ceramic electrode materials on which there is a layer of solid (mixed glass/polycrystalline) and/or liquid coal slag. Above the slag layer is the gaseous plasma. Current flow may be diffuse, or there may be arcing. Since the conduction mechanism in slag is largely ionic, while electrodes are electronic conductors, polarization layers will develop; and electrochemical degradation can occur. To eliminate problems of arcing and electrochemical degradation we must find electrode materials which are compatible with slag conduction mechanisms or which can resist arc erosion. Not all potential electrode candidates have been adequately characterized; and the charge carrying species in slags have not even been adequately identified. Nor have their "transferance numbers" and diffusion coefficients been measured. This is an area which is especially appropriate for work by physicists.

On the cathode wall, the intrinsic thermionic work function of a slag layer may be too high to allow needed operating current densities ( $1-2$ amp. $\mathrm{cm}^{-2}$ ) except at operating temperatures higher than anticipated for the channel. There is now strong evidence (Anderson et al, 1981), however, that the presence of potassium vapor pressure in the plasma layer over the electrode can lower the work function sufficiently to allow the required current densitites. Work needs to be done to measure the actual thermionic work function of these materials under an applieg

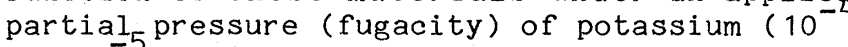
to $10^{-5}$ atm) in order to simulate the MHD environment.
Also on the cathode wall, simultaneously with the development of a polarization layer, a spatially periodic shorting occurs between electrodes along the wall (Demirjian, 1978). There is not sufficient information about this layer to indicate whether the shorting is due to the concentration of cations in the polarization layer, or to a more complex interaction between an electrode and its neighbors, the magnetic field, and the plasma current. But concentrations of these species in a normal slag have been shown to enhance the slag conductivity substantially (Pollina et al, 1980a, 1980b).

In addition to identifying the conducting species in the slag and finding suitable electrode materials, the slag layer conductivity must be in an electrically suitable design range (typically $10^{-2}$ to $10^{1}$ onm ${ }^{-1}{ }^{-1}$ ) for the generator to function properly (Rosa, 1971). Thus it is necessary to measure the conductivity of a slag layer as a function of composition and temperature. In addition to the study of the bulk property, it is also useful to know how the conductivity would change at the boundary layers between electrode and slag, where the chemical composition might be changed because of ionic conduction.

Finally, the slag properties may be modified to be more favorable to the MHD system by the addition of oxides for the purpose of changing either the conductivity or the conducting species.

On the slag-plasma interface ( $1500 C)$ on the anode side of the channel, it has been demonstrated that an ion current of $\mathrm{K}^{+}$is easily obtained going from the slag into the plasma layer. At the anode-slag interface, however, the polarization boundary layer produces a very low conductivity region in the slag because of the removal of cations such as $\mathrm{Fe}^{3+}$ and $\mathrm{K}^{+}$from that layer. This low conductivity layer is thought to be the cause of arcing on the anode. If so, it would explain why anode arcing has been such an intractable problem so far. This is further substantiated by the fact that anode arcing is greatly reduced by the use of iron anodes (Koester, 1978) which act as sacrificial electrodes in contributing themselves as charge carriers in the slag, thus eliminating the low conductivity iron-depletion layer from near the anode. This, however, is not a satisfactory solution to the problem, because the electrode would be quickly exhausted.

One can see from the above that the 
problem of electrical conduction through a layer of molten oxides is formidable, but one which is well suited to work by physicists. The experimental needs are for thermionic emission, charge carrier identification, and transference number measurements. These should be carried out to simplify the search for suitable electrode materials. Once these measurements have been done, then the solutions to the electrochemical equations can be found, and the nature and behavior of the polarization layer and its effect on electrochemical degradation of the electrode material investigated. The goal is to find electrode materials having lifetimes compatible with the normal outage schedule of a power plant, and which can operate almost continuously at $1 \mathrm{amp} / \mathrm{cm}^{2}$ current densities within that lifetime.

The development of advanced superconducting magnet technology is necessary to attain the high magnetic field strength $(6-7 \mathrm{~T})$ over the volume of an MHD channel, $14 \mathrm{~m}$ long by $3 \mathrm{~m}$ diameter (Marston and Thome, 1980).

\section{Recommendations}

- We recommend expanded and strengthened effort on computer simulations (at the atomic level) of interactions between the lattice and "foreign" atoms. Theoretical modeling of $\mathrm{Fe}-\mathrm{H}$ lattice interactions is a case in point.

- We recommend surface science studies of the manner in which hydrogen gains entry to the lattice.

-We recommend further theoretical investigation of crack nucleation and growth, taking into account chemical effects. The possibility should be investigated that some types of chemical environment might strengthen the material at a crack tip.

- We recommend intensified study of all aspects of solid particle erosion -including the mechanisms of material removal, the dependence on kinematical variables and temperature, and the dependence upon particulate properties. We invite attention especially to the importance of studying threshold effects, as they may provide the key to viable systems design.

- We recommend broadened and systematic studies of the transport of minor oxidants and substrate atoms through protective oxide films, with special effort to understand the pathways involved.

- We recommend the use of multicomponent diffusion theory to understand the cooperative relationship between sulfide and carbide formation at the substrate-film interface and their subsequent oxidation and consequent disruption of the protective film.

-We recommend use of the methods of surface physics to study the mechanisms by which alkali sulfates attack metal surfaces and their protective films and the mechanisms of scale formation and spallation.

- We recommend systematic study, including use of surface physics techniques, of phenomena that can be induced by deliberate surface modifications such as coatings, ion implantation, and other chemical treatments.

- We recommend fundamental studies to e 1 ucidate $t h e$ n a t u re of "erosion-corrosion" which at present is poorly understood.

- We recommend an effort to improve and extend the techniques of non-destructive evaluation for structural ceramics -- improvement, for example, of acoustic analysis techniques.

- We recommend fundamental studies of subcritical crack growth, including the use of thermodynamics and rate-process theory.

- We recommend statistical modeling of failures based on fundamental principles in order to provide a physical basis to the present empirical design methodology.

- We recommend increased efforts to complete the phase diagrams that are critical for understanding refractories in slagging environments. These are systems of $4-9$ components; and development of computational techniques to deal with such systems should be encouraged and enhanced.

- We recommend that research on the kinetics of refractory dissolution by slags be strengthened and encouraged -with specific attention to diffusion pathways or mechanisms, and to the respective roles of grain boundaries and matrix phase components in resisting dissolution. In the same vein, we 
recommend a quantitative study of the diffusion through the structure, and subsequent evaporaton and loss, of essential chemical species (i.e. binder, etc.), and an analysis of the consequences for the integrity of the composite refractory under long term exposure to the process environment.

- We recommend study of the interaction of refractory microstructure -- specifically, porosity -- with the growth or arrest of cracks.

- We recommend systematic research into the phenomenology of refractory modification and failure attendant upon thermal cycling.

- We recommend research to identify the charge carriers and to determine transport parameters for slags, as well as studies of electro-chemical interactions in the slag-electrode system.

-We recommend research to establish the systematics of wetting of refractories by slags.

- We recommend that efforts be encouraged to improve quantitative measurements of physical properties (heat capacity, thermal conductivity, vapor pressure, etc.) of refractories at high temperature under realistic or in situ conditions, as well as under laboratory conditions.

\section{References}

Antill, J. E., M. J. Bennett, R. F. A. Carney, G. Dearnaley, F. H. Fern, P. H. Goode, B. L. Myatt, J. F. Turner, and J. B. Warburton, 1980. Corros. Sci. 16, 729.

Anderson, J., W. F. Anderson, M. A. Wilson, and G. J. Lapeyre, 1981, "Thermionic Emission of Some Synthetic and Natural Slags", Montana State University, published by Montana Energy Institute, Butte, Montana 59701.

Bakker, W., 1978a, "Refractory Applications in Gasifiers", 10th Synthetic Pipeline Gas Symposium, pp. 307-336, American Gas Assoc., Chicago, Ill.

Bakker, W., 1978b, "Refractory Applications in Coal Gasifiers" in 3rd Annual Conf. on Materials for Coal Conversion and Utilization, Oct. 10-12, 1978 . Gaithersburg, Md., available from NTIS as DOE publ., CONF-781018.

Bates, J. L., 1970, Thermal Conductivity of
"Round Robin" Uranium Dioxide, BNWL-143, Battelle, Pacific Northwest Laboratories, Richland, WA 99352.

Byker, H. J., R. E. R. Craig, I. Eliezer, N. Eliezer, R. A. Howald, P. Viswanadham, and R. Woodriff, 1980, "Activity Coefficients in Seeded Coal Slag and Their Effects on Vaporization and Crystallization Equilibria" in $7 \mathrm{th}$ International Conference on MHD Electrical Power Generation.

Coffey, J. M. and M. J. Whittle, 1981, Phil. Trans. Roy. Soc. (London) A 229, 93.

Collins, R. A., S. Muhl, and G. Dearnaley, 1980, Phil. Trans. Roy. Soc. (London) A 295, 331.

cosMAT, 1975, Materials and Man's Needs, Summary Report of the Committee on the Survey of Materials Science and Engineering, National Academcy of Sciences, Washington, D.C.

Demirjian, A., S. W. Petty, and A. Solbes, 1978, 17th Symposium on the Engineering Aspects of MHD, C. H. Kruger, Ed., Stanford University, March 27-29, 1978, p.D.1.1, available from Dept. of Mech. Eng., U. Miss., University, Miss. 38677.

Edison Electric Institute, "Report on Equipment Availability for the Ten Year Period", 1965-1975 AND 1966-1976, report 非 5-50 and 76-85, Edison Electric Institute, Washington, D.C. 20036.

Eliezer, I., N. Eliezer, Reed A. Howald, M. C. Verwolf, 1978, J. Phys. Chem. 82, 2688.

Evans, A. G. and A. Rame, 1979, "High Temperature Failure Mechanisms in Ceramics", Lawrence Berkely Laboratory, Report LBL 8692.

Finnie, I., 1979 , Conference on Corrosion/Erosion of Coal Conversion System Materials, National Association of Corrosion Engineers, Houston, TX, 1979, pp. 429-443.

Freimann, S. W., J. J. Mecholski, W. J. McDonough, and R. W. Rice, 1978, "Effect of Oxidation on the Room Temperature Strength of Hot Pressed $\mathrm{Si}_{3} \mathrm{~N}_{4}-\mathrm{MgO}$ and $\mathrm{Si}_{3} \mathrm{~N}_{4}^{-\mathrm{Z}_{\mathrm{O}} \mathrm{O}}{ }^{\prime \prime}$ in Ceramics for High Performance Applications II, Brook Hill Publ. Co., Chestnut Hill, MA, p. 1069ff, J. Burke, E. Lenoe, and R. Nathan Katz, Eds.

Fuller, E. R. Jr., and R. M. Thomson, 1978, in Fracture Mechanics of Ceramics, Vol. 4, Ed. by R. C. Bradt, D. P. H. Hasselman, and F. F. Large, Plenum, N.Y., 
pp. 507-548.

Gehlen, P. C., J. R. Beeler Jr., and R. I. Jaffee, 1972, Eds.., "Interatomic Potentials and Simulation of Lattice Defects", Plenum Press, N.Y.

Hill, V. L., et al., 1979, Symposium 89, American Chemical Society, Washington, D. C.

Howald, Reed A., Isaac Eliezer, P. Viswanadham, 1979, J. Am. Ceram. Soc. 62, 134.

Hutchings, I. M., 1979, Conference on Corrosion/Erosion of Coal Conversion System Materials, National Association of Corrosion Engineers, Houston, TX, 1979, pp. 343-428.

Kennedy, C. R., 1979, "Refractories for Application in Slagging Gasifiers" in 4th Annual Conference on Materials for Coal Conversion Utilization, Oct. 9-11, 1979, Gaithersburg, Md., available from NTIS as DOE publ., CONF-791014.

Kingery, W. D., H. K. Bowen, and D. R. Uhlmann, 1976, Introduction to Ceramics, 2nd ed., J. Wiley and Sons, Inc., N.Y.

Koester, J. K., and R. A. Perkins, 1978, in Fourth US/USSR Colloquium on MHD, published by DOE, available from NTIS, Washington, D.C., pp. 603-634.

Lawn, B. R. and T. R. Wilshaw, 1975 , "Fracture of Brittle Solids", Cambridge Univerity Press, Cambridge.

Leonard, R. J. and R. H. Herron, 1972, Ceramic Bull. 51, 891.

Levin, Ernest M., Carl R. Robbins and Howard F. McMurdie, 1964, 1969, 1975, Eds., Phase Diagrams for Ceramists American Ceramics Society, Columbus, Ohio, 1964 and Supplements (1969 and 1975).

Mangels, J. A., 1978, "Development of Injection Molded Reaction Bonded $\mathrm{Si}_{3} \mathrm{~N}_{4}$ " in Ceramics for High Performance Applications II, Brook Hill Publ. Co., Chestnut Hill, MA, edited by J. Burke, E. Lenoe, and R. N. Katz, pp. 113-130.

Marston, P. G., and R. J. Thome, 1980, in Proceedings 7 th International Conference on MHD Electrical Power Generation, J. F. Louis, Chairman, MIT, Cambridge, MA, June $16-20,1980 \mathrm{pp}$. 450-458.

Muan, A., and E. F. Osborn, 1965, Phase Equilibria Among Oxides in Steelmaking, Addi son-Wesley, Reading, MA.

Muan, A., 1979, in 4th Annual Conference on Materials for Coal Conversion Utilization, oct. 9-11, 1979, Gaithersburg, MD., available from NTIS as
DOE publ., CONF-791014. p IV-44ff.

Nelson, G. A., 1969, Action of Hydrogen on Steel at $\mathrm{High}$ Temperature and $\mathrm{High}$ Pressures in Interpretative Report on Effect of Hydrogen in Pressure-Vessel Steels. Welding Research Council Bulletin \#145, N.Y., Oct., 1969, pp. $33-42$.

Paskin, A., A. Gohar, and G. H. Dienes, 1980, Phys. Rev. Letters, 44, 940-943 and references therein.

Perkins, R. A., 1979, Materials Problems in Fluidized-Bed Combustion Systems IV-Corrosion Chemistry in Low-Oxygen Activity Atmospheres, Report EPRI-FP-1280, Electrical Power Research Institute, Palo Alto, CA.

Pettit, F. S., R. H. Barkalow, and J. A. Goebel, 1980, Materials Problems in Fluidized-Bed Combustion Systems, High Temperature Erosion-Corrosion by High-Velocity $(200 \mathrm{~m} / \mathrm{sec})$ Particles in Report EPRI-CS-1448, Electric Power Research Institute, Palo Alto, CA.

Pollina, R. J., Raymond Larsen, and AI Kumnick, 1980a, "Coal Slag: Its Electrical Properties and Some Mechanical Effects on MHD Ceramics" in Energy and Ceramics, Ed., P. Vincenzini, Elsevier, N.Y.

Pollina, R. J., Raymond Larsen, and David Westpfahl, 1980b, J. Noncrystalline Solids $40,197$.

Pollina, $\bar{R}$. J., and R. Smyth, 1981 , "Refractories for High Temperature MHD Applications" in Proceedings of the International Colloquium on the Use of Refractory Oxides for High Temperature Energy Conversion Processes, Toronto, Canada, July 16-19, 1979, High Temperature Science (in press).

Rau, C. A., and P. M. Besuner, 1981, Phil. Trans. Roy. Soc. (London) A 299, 111.

Rehn, I. M., 1981, "Review of Recent Developments Concerning Scale Formation and Exfoliation of Steam Generator Tube Materials", EPRI Report on Project 644-1 (in press).

Reid, W. T., 1971, External Corrosion and Deposits, Elsevier, N.Y.

Rice, J.R. and R. M. Thomson, 1974, Phil. Mag. 29, 73.

Rosa, R. J., 1971, "Design Considerations for Coal-Fired MHD Generator Ducts", in 5 th International Conference on MHD Electrical Power Generation, Munich, 1971. 
Sims, C. T., and J. E. Palko, 1977, "Surface Stability of Ceramics Applied to Energy Converion Systems in Proceedings on the Workshop on Ceramics for Advanced Heat Energies, Jan. 24-26, 1977, Orlando, Florida, ERDA, CONF 770110, available from NTIS.

Smeltzer, W. W., D. P. Whittle, and D. J. Young, 1976, in Properties of $\mathrm{High}$ Temperature Alloys, Electrochemical Society, pp. 134-153.

Stringer, J, and S. Erlich, 1976, ASME Paper 76-WS/CD-4.

Stringer, J., 1977, Ann. Rev. Matl. Sci. 1 , 477.

Thomson, R. M., 1980, J. Matl. Sci. 15, 1014.

Tien, J. K., O. Buck, R. C. Bates, and S. Nair, 1980, Scripta Met 14, 583.

Tomkins, B., 1981, Phil. Trans. Roy. Soc. (London) A 299, 31.

Townes, H. W. , R. J. Pollina, R. Larsen, K. Kiddo, R. Smyth, G. E. Youngblood, and W. C. Seymour, 1981, "Operability and Materials Performance of a Regenerative Heat Exchanger During $1000+$ Hours of Operation" in l9th MHD Symposium, Tullahoma, TN, June, 1981.
Uy, J. C., R. M. Williams, and M. V. Goodyear, 1978, "Study of the Processing and Bond Strength of Hot-Pressed $\mathrm{Si}_{3} \mathrm{~N}_{4}$ ", in Ceramics for High Performance Applications II, Brook Hill Publ. Co.. Chestnut Hill, MA, edited by J. Burke, E. Lenoe, and R. N. Katz, pp. 131-150.

Watt, J. D., 1959, BCURA Bul1. 23, 49-69.

Weibull, W., 1951;, J. of Appl. Mechanics, $18,293-297$.

Wells, A. A., 1981, Phil. Trans. Roy. Soc. (London) A 299, 19.

Whittle, D. P., 1972, in High Temperature Corrosion of Aerospace Alloys, Ed. by $\mathrm{J}$. Stringer, et al., Proceedings of the Advisory Group for Aerospace Research and Development, AGARD-C P-120, pp. 173-199, available from NTIS.

Williams, R. M. and J. C. Uy, 1978, "Ceramic Material Characterization", in Ceramics for High Performance Application II, Brook Hill Publ. Co., Chestnut Hlll, MA, edited by J. Burke, E. Lenoe, and R. N. Katz, pp. 151-178.

Wilson, W. D., 1980, in Arpa Workshop, La Jolla CA, July 16-18, 1980, available from NTIS. 


\section{E. SIZE-DEPENDENT PHENOMENA}

\section{Introduction}

Coal utilization entails particle size reduction (usually by grinding) as well as interactions with fluids, whether these are native to the coal or are added for the purpose of transportation or during size reduction. Some fraction of the energy input for coal processing and utilization is expended in inefficient grinding processes. The understanding of the fundamental mechanisms involved is rudimentary. There is a dearth of measurement on stress-strain relations and on the swelling behavior with various fluids (e.g. $\mathrm{H}_{2} \mathrm{O}$, methanol), while the role of grinding aids is not understood. Little is understood about fracture mechanisms or about crack initiation and propagation in the coal particles under grinding stresses. Chemical comminution, currently an expensive particle size-reduction technique, is poorly understood. Similarly, the interaction between coal particles and fluids such as water or methanol in slurry formation has not been sufficiently studied. Especially lacking are data relative to high concentration slurries. Reduction of coal particles to sizes of the order of a few microns, in a cost effective manner, would allow the direct utilization of coal in boilers designed for residual fuel oil, without major retrofitting. On the other hand, the ability to control coal particle size better could improve efficiency by the production of fewer coal "fines", i.e. particles of a few microns in typical dimension. Finally, the ability to separate coal particles by techniques not dependent on density differences is highly desirable, especially in the finer particle sizes (see below).

\section{Coal preparation}

Current "coal preparation" practices in the U.S. involve size reduction, mechanical cleaning, and thermal drying of coal for the purposes of maximizing recovery of coal heat content in boilers; washing coal fines; optimizing usage of scarce water resources; and reducing sulfur in the final product. The coal preparation sequence involves four steps: (1) size reduction -- (Progressive size reduction of coal particles is a most significant process in the over all preparation or "beneficiation" of coal prior to its use. The most important procedures for size reduction are the following: primary breakage of mined coal ( 8 " to $3^{\prime \prime}$ ), secondary crushing to a maximum size $(1-1 / 4 ")$, pulverization $\left(1 / 8^{\prime \prime}\right)$ and grinding $\left.(\sim 1 \mathrm{~mm}).\right)$; (2) "sizing" of coal, i.e., screening to a desired range of sizes; (3) cleaning to remove dust and non-coal materials by dry or wet washing techniques -- (Dry washing involves blowing air for removing small particles. Wet washing may utilize floating the coal in a "dense medium" water/magnetite suspension* in which the impurities sink; or froth flotation, whereby chemicals are added to increase the hydrophobicity of coal, which attaches itself to air bubbles and finally is skimmed of $f$ the top as a froth. It should be noted that all wet washing methods are undesirable because they add moisture to the coal, an energy expensive process since the water will have to be removed later.); (4) drying of coal for transportation or other end uses -- (Drying normally utilizes hot air streams in various configurations including fluidized beds. Oxygen may also be used, promoting at the same time oxidation of the inorganic sulfur (Lowry, 1945; U. of Oklahoma, 1975).)

Coal preparation, or beneficiation, as practiced currently is generally recognized to be an "energy-inefficient" process and, therefore, subject to possible major improvements. Breaking, sizing and washing processes for coal consume $0.5-1.0 \%$ of the energy produced ( 4 x $10^{9} \mathrm{Btu}$ 's per $10^{\text {Btu's }}$ processed) (U. of Oklahoma, 1975). The question of defining the efficiency of size reduction, however, is in itself a difficult one and warrants further research. The definition as currently used (Gaudin, 1939) utilizes the increase in surface energy involved in creating smaller particles to specify the useful energy input, and defines the remaining ( $(29 \%)$ of the energy input that goes into "heating" the sample as being wasted. However, there is a fundamental question as to whether at least some of the "wasted" energy is involved in unavoidable

*Typically, finely ground magnetite is mixed with water to make such a dense medium suspension with a specific gravity that allows coal to float, while heavier mineral impurities sink. The magnetic moment of the magnetite is used to recover it for reuse. 
deformation and chemical bond breaking within the coal. (See discussion on pp. 48-49 of Orr $(1966)$. .)

Currently coal is "prepared" by various mechanical means for gross cleaning and preparation of feeds of maximum particle size for power generation. However, coal preparation or beneficiation for the purpose of creating particles free of deleterious impurities, in an efficient and cost-effective manner, is becoming even more important. Size reduction, if carried far enough, breaks the particles to small enough size to liberate most of the organic matter from pyritic sulfur and other undesirable inorganic components. (Of course, the problem remains that after "liberation" one must be able to separate the particles).

In addition to breaking apart the organic and inorganic matter, grinding may also effect a separation of the various carbonaceous components of coal, the macerals. If one then could separate the constituent macerals from each other, one could have the means of preparing coal feeds of highly selective characteristics that not only may be free of environmentally deleterious components, but also may be better feeds for gasification, liquefaction or direct combustion. It is established that different macerals not only have different physical properties (friability, density, hardness, etc.) but also greatly differing chemical reactivities. For example, whole vitrinites and resinites may undergo liquefaction quite readily; fusinites on the other hand are resistant to liquefaction under ordinary conditions (Petrakis, 1981). However, the liberation and subsequent separation of various carbonaceous components from each other as well as from the lighter inorganic components is a difficult problem. This is true because the primary property (i.e.,.density) used for separation is not very different among the macerals and lighter inorganics. Furthermore, since the particle size will be very small and the surface chemistry of macerals is unknown, froth floation is not yet a practical separation technique. Thus, there is a challenge to develop techniques that will separate the macerals from each other and the lighter inorganics once they have been liberated from the coal.

Size reduction by grinding is mainly carried out in inefficient ball mills. Liquids $\left(\mathrm{e} . \mathrm{g} \cdot, \mathrm{H}_{2} \mathrm{O}\right)$ are often used as grinding aids. In the long run this may increase the inefficiency of the overall process, since the water may have to be removed again before the ground coal can be used.

Another problem is that all comminution processes -- crushing, grinding, pulverization -- produce a wide range of particle sizes that include ultrafine particles $(<1 \mu \mathrm{m})$. This is undesirable because the ultrafine particles are lost (washed away) in the preparation process. It has been estimated that $10 \%$ of processed coal may be lost due to the making of particles $<0.5 \mathrm{~mm}$ in size during coal preparation (Soo, 1976). There exist, on the other hand, some arguments in favor of producing uniformly microscopic coal particles (Peters, 1965; Herbst, 1978). Such particles, it is claimed, can be burned directly in a diesel engine since coal particles $<5 \mu \mathrm{m}$ behave like oil droplets (Foo, 1979)*. Such a development has also the distinct advantage that it would allow the direct combustion of coal in oil boilers without major retrofitting. A phenomenon of practical importance, that is of particular interest to physicists, is that at a size of 50 microns and less, grinding rates slow abruptly. The hypothesis is that there is some cohesive force acting between the particles that causes them to agglomerate. The physical questions involved seem to be evocative of questions that arise in polymer physics for forces within collections of particles in this size range. For discussion of such effects in coal see Austin and Bagga (1979). (In grinding of aluminium powder, a rewelding may be involved (Meloy, 1981).)

Opportunities exist for significant contributions to the better understanding and improved practice of size reduction technology, which is generally regarded as not very sophisticated. The physics community has the opportunity to assist several aspects of coal utilization through specific studies that may include the following.

- Define the physical structure of coal and relate significant

* In the New York Times of June 4 , 1981, a report appeared that General Motors had developed a experimental vehicle using a turbine engine to burn coal particles of three micron average size ( $J$. Holusha, page D4, New York Times, June 4, 1981). 
properties to size reduction processes. This is a particularly important area for the solid state physics community. After years of neglect, only now do there appear to be some serious efforts to use in coal research the powerful experimental and theoretical tools that were developed in conjunction with more homogeneous, better defined solid state systems. The challenge here will stem primarily from the highly complex and inhomogeneous nature of coals. Yet, an understanding, for example, of fracture initiation and crack propagation might be of much help in designing energy efficient size-reduction processes. (See Sec. IVD2b for a general discussion of crack nucleation and growth in materials.) There appears to be either uncertainty or lack of information as to the brittleness and plasticity of coal, the nature of elasticity, the nature and role of internal and surface inhomogeneities (occlusions, grains, etc.) and the significance of local deformation. One immediate and very far-reaching implication of the better understanding of the fracture physics of coal would be the design of energy-efficient mills (Luckie, 1980).

- Define the nature of the adhesion between organic and mineral

matter as well as between macerals; relate the nature of these bonds to size reduction processes. There is little information on the nature of the bond between the inorganic and carbonaceous components of coal or on bonds between the various macerals. Different macerals are known to have quite different physical characteristics (hardness, friability, swelling) which can affect the grinding process. But, these characteristics are neither defined precisely nor is it known exactly how they affect size reduction. Empirical correlations have been attempted, mostly with the gross parameter of coal rank. Improved understanding of the physical variations among coal constituents and of their roles in coal utilization is very desirable. For example, (Oder, 1980) it has been observed that very light components are crucial in determining certain engineering variables. Plots of volatile matter vs specific gravity of ash-free carbonaceous components of coal are critical phenomena type curves, i.e. the plot of volatile matter versus specific gravity is similar to that of ordered moment versus temperature for a Heisenberg ferromagnet, dropping off sharply at some critical specific gravity. This type of behavior suggests that light components of coal may be the critical fractions in determining engineering behavior that is dependent on volatile matter. This behavior is one of several examples which indicate the importance of finding relationships between chemical or physical structure of coal and size reduction, and which also indicate the potential value of developing techniques to prepare feeds for industrial processes that concentrate selected components (e.g. readily liquefiable macerals) as well as accomplishing size reduction. Another very suggestive fact (Petrakis, 1981) is that the concentrations of paramagnetic centers (organic free-radicals) in residual free-radical concentrations correlate quite well with the propensity of the macerals to liquefy. If free-radical chemistry proves to be the key to liquefiability, then the development of combined size-reduction maceral-segregation processes could be a major contribution to liquefaction technology.

- Develop energy-efficient grinding processes. All current sophisticated grinding techniques are inefficient. What are the fundamentals of grinding _- in coal specifically? Can processes be developed that allow the selective liberation of the inorganic mineral from the organic matter, or such that there is a minimum of ultrafine particles? Similarly, can selective grinding be carried out that selectively pulverizes macerals of a single kind rather than all macerals indiscriminately. Can the efficiency of breaking-up of coal be increased by novel approaches such as explosive shattering, shock waves, high intensity acoustic energy (see IVB6), or electric shock? An ad hoc committee of the National Academy of Sciences has collected data on efficiency of grinding processes (Herbst, 1978), and a critical evaluation of the data is being prepared. Modeling of such data could lead to significant theoretical and experimental examination of this problem.

- In summary, size reduction is likely 
to increase in importance as an aspect of coal preparation prior to end use. Upon fine grinding, mineral matter is separated from the organic matrix. For larger particles, separation is effected by taking advantage of the density differences between organic and inorganic matter. This is the extent of current coal preparation practices. Growing demand for environmentally acceptable coal feeds, as well as for feeds tailored to specific coal conversion processes or direct combustion, make it important that we improve our fundamental understanding of the phenomena relating to size reduction.

\section{Mechanical Properties of coals}

The mechanical properties of coal play a significant role in all aspects of coal utilization, and yet there is a dearth of reliable measurements and very little fundamental understanding of the relationship between the mechanical properties of coal and its physical and chemical structure. The determinations of mechanical properties of coal have been limited in number and results appear to differ according to the methodology employed (Larsen, 1978).

Conventional testing for properties relevant to coal preparation is rather primitive, and it involves such tests as free swelling index (ASTM test for a given coal's suitability for coking or other uses); friability (ASTM test to measure size reduction during handling operation); and the Hardgrove Grindability Test (an empirical correlation with ease of grinding) (Boughman, 1978). The existing theoretical consideration of the dynamics of coal fracture requires information on how different particles are stressed by forces in a mill; the frequency with which particles are stressed; and tensile regions produced in each particle. The incomplete understanding of the phenomena involved has led to the following observation: "When one considers the complexity of describing the dynamic applied forces in (various mills), the complexity of the distribution of flow sizes in solids and the difficulty of solving stress-strain equations for irregular shapes and glancing blows, it is not surprising that the designer (of grinding equipment) has to rely largely on empiricism in the prediction of mill performance" (Boughman, 1978).

There is general agreement that bituminous coals are polymeric in character (Larsen, 1978) and that they contain a number of smaller (MW 1000) extractable molecules. Both invasive and non-invasive techniques have been utilized to investigate the macromolecular structure of coals. Invasive techniques, utilizing solvents such as pyridine, include extraction, separation, diffusion and swelling of coals. Non-invasive techniques include mostly mechanical testing such as stress-strain behavior at low deformation and ultrasonic determination of shear and compressive moduli (Larsen, 1978; Lucht and Peppas, 1981). The attempt to quantify these data and determine molecular parameters from elasticity measurements has not yet succeeded.

The nature of the macromolecular network has been discussed recently; and bituminous coal is thought to consist of covalently cross-linked macromolecules rather than macromolecules that are held together by van der Waals forces or H-bonding (Larsen, 1978). This conclusion is based on the observation that strain vs time curves level out after an initial increase, the leveling value being determined by the nature of the cross links. If the macromolecular structure were not cross-linked, then one would predict that -since there is no internal limit to the flow due to a constant stress -- the deformation would continue to increase with time. Thus the polymeric picture of coal is strengthened by consideration of its bulk plastic properties. But there is disagreement about the size distribution of the constituent macromolecules, the degree of cross-linking and the topology of the macromolecular network, especially as it is affected by the pores of the overall physical structure (Larsen, 1978). The need for, and the importance of, extensive and reliable measurements of the mechanical properties of coals and coal constituents seem obvious.

The actual stress-strain measurements on coal are quite limited, and they have been obtained with large samples (Van Krevelan, 1965). As such, they have been criticized in that cracks and other macroscopic inhomogeneities may have influenced the results. Nevertheless, data exist which show that strain-time curves for bituminous coals do reach a constant value; for both bituminous 
coals and pure clarains*, only $1 \%$ or less of the total strain is irrecoverable, as would be expected from a 3-dimensional macromolecular, covalently bonded structure (Larsen, 1980). But such data are sparse.

The area of mechanical properties of coals presents many opportunities for contributions from the physics research community. Among the many opportunities or needs, we consider the following as the most important.

- To improve fundamental understanding of the mechanical properties of coal. Such an understanding is important not only for size reduction processes as discussed earlier, but also in mining and transportation (support strength of pillars in "long-wall" and "room-and-pillar" mining patterns). Understanding the mechanical properties of coal would also aid in the development of abrasion resistant vessels and equipment in all aspects of coal utilization, from mining to grinding to transportation and conversion. There is need to understand how the mechanical behavior is affected by, and relates to, the chemical structure (heteroatomic species, bridging moities between macromolecules, polar chemical functionalities) and the overall physical structure (porosity, permeability of coals). Solvent (especially water, methanol, ammonia) diffusion and interaction with coal, along with solvent swelling behavior, should be studied; the behavior under stress should be studied as well, since these data can be used to infer the cross-link parameters. The most important parameters are (a) the number average molecular weight per cross link, $\overline{\mathrm{M}}$, and (b) the cross linking density $\rho_{x}$ expressed as moles of cross links per unit volume. Perhaps something can be learned from the corresponding physics of synthetic and natural polymers. Appropriate statistical theories of

*British "type" classification for bright, finely laminated material in bituminous coal (Stopes, 1919). polymer network elasticity must be developed, accounting for both entropic factors and intermolecular forces. The relative contributions of macromolecular entanglements will need to be examined as they effect elastic restoring forces.

- To determine shear and other moduli and their dependence on coal rank, maceral and coal primary (chemical) structures; to determine the applicability and utilization of various techniques (e.g., sound velocity measurements) to the determination of such moduli. Work is needed to bridge the discrepancy of several orders of magnitude in $\bar{M}$ as determined by solvent swelling compared to that determined by stress-strain measurements. The discrepancy has been attributed to differences in experimental procedures; various arguments also have been advanced to account partly for the discrepancy as being due either to intermolecular obstructions in the network or to swollen network effects.

- To examine the statistical theory of coal network elasticity and to inquire what statistics the coal macromolecules may obey, because coal macromolecules may be neither long enough nor flexible enough for Gaussian statistics to apply. Entanglements, intermolecular forces, cooperative network effects, and the role of small coal extractable molecules or solvents entering the coal, are important effects in the mechanical behavior of coal and need to be accounted for (Larsen, 1978; Lucht and Peppas, 1981). There is a particularly significant need for experimental work to reexamine the extraction and swelling behavior of bituminous coals, emphasizing the thermodynamic interaction parameter, the cross linking parameters $\bar{M}$ and $\overline{\rho x}$ and the molecular weight distribution of the "extractable coal" portion.

- To investigate the mechanism of fracture initiation and propagation in coal. Conventional theory suggests fracturing would be 
determined by critical stress, rate of loading and temperature (among other factors). However, there is very little information available that is specific to coal.

\section{Chemical comminution}

Chemical comminution of coal is an alternative and conceivably attractive process for size reduction and concommitant liberation of mineral matter. This involves the treatment of coal with a reagent such as concentrated aqueous ammonia solution. This treatment brings about the selective breakage of coal particles along the boundaries between macerals and mineral components, as well as along bedding planes.

There seems to be general agreement that chemical comminution to a given particle size generally produces greater liberation of pyritic sulfur than mechanical grinding, although the two processes appear to yield comparable liberation of mineral matter that produces ash. Experiments with an Illinois No. 6 bituminous coal have shown chemical fracture ( $4.5 \% 150$ microns) liberates considerably more pyritic sulfur than mechanical crushing even to a considerably finer size (22\% 150 microns) (Wheelock, 1977).

Another possible advantage of chemical comminution appears to be a narrower particle size distribution, with smaller production of ultrafines. Thus the economics of such an approach to size reduction might be favorable, although there are other factors (processing time and cost of reagents) that mitigate against favorable economics (Datta, 1976). A reagent cost as low as $\$ 10$ or $\$ 15$ a ton is quite significant compared to the price of a ton of coal (say $\$ 25$ to $\$ 50$ a ton). Also, there is some evidence that -- at least with ammonia as the comminuting agent -- there may be a reaction taking place which increases the organically bound nitrogen content of the comminuted coal, an adverse effect.

Treatment of coals with chemicals, such as ammonia, fragments coals selectively along bedding planes and at boundaries between macerals and mineral matter. The understanding both of the phenomenology and of the mechanisms involved and, therefore, of the potential of chemical comminution for technological exploitation on a cost effective basis, is rudimentary. But the potential may be quite significant. One would need to understand the nature of the various bonds and interactions that are affected in this process. Particularly important is the need to identify those characteristics of the reagents that are effective in comminution. In addition to ammonia, there are others (methanol, amines) that also comminute coal, although not as effectively. All these compounds are highly polar, with oxygen or nitrogen functionalities and a lone non-bonding pair of electrons. Such types of compounds can swell and dissolve in coal even at low temperatures.

- Diffusion of the comminuting agents in coals, the swelling of coal, the nature of the surface interaction between the coal and the comminuting agent, and the resulting crack initiation and/or propagation, all need to be investigated both theoretically and experimentally. Some preliminary theoretical discussion of the comminution mechanism does not account for the comminution by gaseous ammonia (Wheelock, 1977, 1981). The techniques of solid state physics, particularly emphasis on such techniques as electron microscopy, could shed light on this important topic; these techniques also might provide some explanation of the fact that chemical comminution is strongly affected not only by the nature of the comminuting agent, but also by such parameters as coal moisture, particle size of coal, pressure, pretreatment (partial drying or evacuation), maceral content, porosity, and permeability of coal. A most significant question is whether there exists a combination of chemical comminution and other size reduction procedures that a) may be more energy efficient than mechanical crushing alone and b) may result in a better controlled particle size distribution.

\section{Coal-oil mixtures}

Coal-oil mixtures for combustion in boilers are receiving considerable research and development attention. Efforts are under 
way in several countries not only to demonstrate the engineering feasibility of utilizing coal-oil mixtures in utility and industrial boilers which were designed for other fuels, but also to develop a fundamental understanding of the behavior of coal-oil mixtures. The research and development efforts address such aspects of the utilization of coal-oil mixtures, as preparation, storage, transportation, and combustion control (Botsaris, 1979; Smith, 1979).

Coal-oil mixtures have been examined periodically in the past, mostly on a trial-and-error basis. Much technical progress was made in achieving slurry stability (i.e. particle suspension and pumpability) through viscosity adjustment. A 1942 program at an Atlantic Company refinery demonstrated that stabilization of suspension to prevent coal settling could be accomplished by using very finely ground coal ( $1 \%$ coal particles $<70$ microns). Up to 40 wt $\%$ slurries were prepared with No. 6 fuel oil, the slurry retaining a viscosity that allowed pumping with preheating $(250-350 \mathrm{cp}$ @ $100 \mathrm{~F})$. To achieve suspension stability requires coal particles $<10 \mu \mathrm{m}$ or, aiternatively, viscosity can be increased by "gelling" with soaps or lime-resin greases. Use of such additives results in thixotropic slurries that are sufficiently viscous to support coal particles. Much of that early work related to slurry formation and stability was empirical, with minimal effort devoted to delineating the fundamental rheological properties and surface interactions of the constituents involved.

In addition to the degree of pulverization, other factors which affect coal-oil slurries are introduction of water as a third major component in the slurry; interaction between coal particles and solvent/carrier; reactivity of lignites; the specific fuel oils used as slurry vehicles; and the coal rank, maceral content, and mineral matter contained in the coal. The effect of these latter specific characteristics of the coal may be especially important in the burning characteristics of the coal-oil slurry.

Grinding in an oil medium (as opposed to dry grinding) and then dispersing pulverized coal to make oil slurries is receiving increased attention. The scheme appears to offer several advantages in that it may minimize surface oxidation during grinding and thereby enhance dispersion, reduce settling, eliminate explosion hazards due to dust being generated in dry grinding, and simplify grinding equipment (. $\mathrm{g}$..' Szego grinding mill). Finally, consideration has been given to transportation of coal in oil slurries; this may be particularly attractive if it eliminates the need for using scarce water, or if coal must be further reduced in size after transportation but prior to combustion.

Properties of coal-oil slurries are affected by the nature of the interaction between coal and the carrier oil. Some important questions relate to stability, determination of viscosity, pumping requirements, and determination and monitoring of composition and stability. Needs exist here also for contributions from physics. For example, difficulties have been reported in attempted viscosity measurements with No. 2 fuel oil mixtures, because these slurries have tended to channel around the viscometer spindles in a Brookfield viscometer. Measurements of these high viscosity slurries are additionally complicated when they are attempted at high temperatures (300F) (Smith, 1979).

\section{- Preparation and stabilization} of high-concentration slurries (50-70 wt $\%$ coal), heat transfer rates, fly ash particle properties and combustion mechanisms are important problems. Smith et. al. have provided a brief introduction to the pertinent fluid mechanics relevant to mixing and slurry stability. Viscosity, evaporation losses, mixing and settling, mixing and power requirements, and maintenance of slurry stability through the use of additives (dispersants or surfactants) are important topics for investigation. Physical means of maintaining the stability of a slurry have also been considered (ultrafine pulverization and ultrasonic agitation).

Rotary viscometric studies have shown that the rheology of slurries below 30 vol \% coal is Newtonian. The behavior of the more concentrated slurries is not particularly well understood, although it appears to depend on shear stress and to be thixotropic. Thickening can require a large shear stress to restore fluid motion, and presents a hazard in transportation (pipeline blockage) as well. 
Emulsification with water appears to ameliorate this problem and also improves combustion. - Sedimentation and filtration theory need to be investigated at high loadings of solids in high viscosity 1 iquids. The coal-oil-water ternary mixtures are particularly important and stand in need of experimental and theoretical investigation. The relative hydrophobicity (or oleophilicity) and hydrophilicity of coals are believed to influence the effects of additives, and therefore the properties of the coal-oil-slurries, but the detailed mechanisms are not understood.

\section{Coal/water systems}

Fundamentals of the interaction of water and coal merit considerable investigation; this interaction is important in most branches of coal technology. For example:

Dewatering is an important aspect of coal preparation. Water (either native or added as a grinding aid) is present in coal which has been ground, but must be removed prior to use. Thermal, mechanical, or chemical means may be used to effect water removal. The interaction is complex and recently has been the object of much study. The diffusion of water, its interaction with coal surfaces and the nature and strength of the bond that forms with coal clearly are important matters for investigation (see discussion in Section III B). The synergistic or antagonistic effects of water and surfactants (or other additives) are also of interest.

Water is particularly significant in the utilization of lignite. Lignite and other low ranked coals may contain up to $40 \%$ water. This high water content gives rise to several problems, among them transportation problems such as "excess baggage of water", freezing, or even spontaneous combustion. It has been estimated that lowering the water content to about $10 \%$ would make lignite economic to use at much greater distances from the mine.

\section{Beneficiation/high gradient magnetic separation (HGMS)}

$$
\text { - It is highly desirable to }
$$

separation technologies for coal beneficiation, that depend on properties other than density. There are many possibilities that can be pursued, and reviewing them all is beyond the scope of this report. However, the possibilities may be illustrated by discussing the case of High Gradient Magnetic Separation (HGMS) (Maxwel1, 1978; Petrakis et al, 1980).

High gradient magnetic separation is a recently developed technique in which micron-size particles contained in a flowing fluid medium can be extracted from that medium. The technique can also be extended to non-magnetic moities provided that they can be made to associate with magnetic seeding materials.

Magnetic separations have been used in mineral processing for many years and in several forms. The basis of the separation process is the force acting upon a magnetic particle in a magnetic field due to the magnetization of the particle and the magnetic gradient in the region about the particle. Typical magnetic separators that have been used commercially, as well as the values for the magnetic gradient contained within each separator, are as follows: wet drum separator employing permanent magnets for processing magnetic ores (gradient 0.1 $\mathrm{kG} / \mathrm{cm}$ ); suspended electromagnetic separators for tramp iron removal (gradient of $1-10 \mathrm{kG} / \mathrm{cm}$ ); wet high intensity separators for processing weakly magnetic iron ores $(100-1,000 \mathrm{kG} / \mathrm{cm})$. HGMS represents an extension of these gradient values, i.e., the gradient contained within an HGMS unit possesses a value 1000-10,000 kG/cm. It is the increased value for the gradient within an HGMS separator that permits, inter alia, the separation of paramagnetic micron size particles from a diamagnetic medium on a large scale. A second attribute of an HGMS separator that is significant is that is permits separation rates that are much greater than those obtained by standard filtration methods. HGMS has already been applied successfully for beneficiation of coal and coal liquid, showing some considerable promise of success (Maxwell et al, 1981). 


\section{Recommendations}

1. Research is needed to improve understanding of the physical structure of coal and to relate measurable/describable properties to the mechanics of size reduction processes.

2. It is desirable to investigate the nature of the "bond" (adhesion?) between organic and mineral matter, as well as between macerals, and to relate the strength of these bonds to size reduction processes.

3. Invent energy-efficient grinding processes. Especially develop models to explain the decrease in grinding rate for particles below $50 \mu$ diameter.

4. Attempt a rational description and classification of the mechanical properties of coal.

5. Determine shear and other moduli and their dependence on coal rank, maceral and coal primary (chemical) structure; and develop new techniques (e.g., sound velocity measurements) for the determination of such moduli.

6. Examine the statistical theory of coal network elasticity and investigate the statistics coal macromolecules may obey, because they may be neither long enough nor flexible enough for the Gaussian statistics of polymer theory to be appropriate.

7. Investigate the mechanisms of fracture initiation and propagation in coal and in coal particles of various size.

8. Diffusion of the chemical comminuting agents in coals, swelling, the nature of the surface interaction between the coal and the comminuting agent, and the resulting crack initiation and/or propagation, all need to be investigated theoretically and experimentally.

9. Mixing and stabilization, heat transfer rates, fly ash particle properties and combustion mechanisms are important problems for the development and use of high-concentration slurries (50-70 wt \% coall).

10. Sedimentation and filtration theory need to be investigated at high loading of solids in high viscosity liquids.
11. Investigate applicability of novel separation/beneficiation technologies that depend on properties other than density (e.g. high gradient magnetic separation).

\section{References}

Austin, L. G. and P. Bagga, 1979, November, preprint.

Baughman, G. L., 1978, Synthetic Fuels Data Handbook, Cameron Engineers, Inc., Denver, CO.

Botsaris, G., et al, 1979, Second International Symposium on Coal-Oil Mixture Combustion, NTIS Conf-791169, Vols. I and II.

Datta, R. S., P. H. Howard, A. Hanehett, 1979, NCA/BCR Coal Conference and Exposition III. Coal: Energy for Independence.

Foo, O. K., et al, 1979, DOE Report Contract No. ET-78-C-01-3179, The Mitre Corp., McLean, VA.

Gaudin, A. M., 1939, see page 184 in Principles of Mineral Dressing, McGraw-Hill, New York.

Herbst, J. A., 1978, "Energy Requirements for the Fine Grinding of Coal in an Attritor", U.S. Dept. of Energy, Final Report Contract No. EY-77-S-02-4560.

Howard, P. H., and R. S. Datta, 1977, Coal Desulfurization: Chemical and Physical Methods, T. D. Wheelock, ed., ACS Symposium Series No. 64, Washington, D.C.

Larsen, J. W., T. K. Green, P. Choudbury, and $E$. W. Kuemmerle, 1981, Advances in Chemistry, Vol. 192 edited by M. L. Gorbatty and O. Ouchi, American Chemical Society, Washington.

Larsen, J. and J. Kovac, 1978, "Polymer Structure of Bituminous Coals" in Organic Structure of Coal, J. Larsen, ed., Symposium Series 71, American Chemical Society, Washington, D.C.

Lowry, H. H., ed., 1945, Chemistry of Coal Utilization, J. Wiley, NY.

Lucht, L. M., and N. A. Peppas, 1981, pp. 28-48 in Chemistry and Physics of Coal Utilization-1980, edited by B. R. Cooper and L. Petrakis, Am. Inst. of Phys., Proceedings Series No. 70.

Luckie, P. T., and L. G. Austin, 1980 , FE-2375-25, DOE Coal Grinding Technology -- A Manual for Process Engineers. 
Maxwell, E., 1978, pp. 98-112 in Scientific Problems of Coal Utilization, edited by B. R. Cooper, Dept. of Energy Symposium Series, available as CONF-770509 from National Technical Information Series.

Maxwell, E., D. R. Kell and, I. S. Jacobs, and L. M. Levinson, 1981, in Chemistry and Physics of Coal Utilization-1980, edited by B. R. Cooper and L. Petrakis, A I P Conference Proceedings No. 70, American Institute of Physics, New York.

Meloy, T. P., 1981, private communication.

Morgan, W. T. A., and N. B. Terry, 1958, Fuel, 37, 201.

Mraw, S. C., and B. G. Silbernagel, 1981, Physics and Chemistry of Coal Utilization-l980, B. R. Cooper and L. Petrakis, eds., Am. Inst. of Phys. Proceedings Series No. 70.

Oder, R. R., 1980, private communication.

Orr, C., Jr., 1966, Particulate Technology, MacMillan, New York.

Parish, B., 1974, A Bibliography of Comminution, Inst. Chem. Engineers, London.

Peters, W., and H. Juntgen, 1964, 1965, Brennstoffe-Chemie, 46, 56, 46, 175.

Petrakis, L., P. F. Ahner, and $\bar{F}$. E. Kiviat, 1980, p. 1260 in Fine Particle Processing, Vol. II, edited by P. Somashundaran, AIME.

Petrakis, L., and D. W. Grundy, 1981, Nature, 289, 476.
Petrakis, L., and D. W. Grundy, 1981, Fuel, $60,120$.

Petrakis, L., and D. W. Grundy, Proceedings Royal Society, accepted for publication 1981.

Smith, R. S., et al, 1979, Second International Symposium on Coal-0il Mixtures Combustion, Dept. of Energy, Conf. 791160 .

Soo, S. L., et al, Annual Review of Energy, J. M. Hollander, ed., Vol. I, 1976.

Stopes, M. C., 1919 , Proc. Roy. Soc. (London) 90B, 470.

University of Oklahoma, The Science and Public Policy Program, 1975, Energy Alternatives: A Comparative Analysis, U.S. Gov't Publication PREX 14.2: EN2, Washington, D. C

Ursu, Ioan, ed., 1975, Energy and Physics, Proceedings of the Third General Conference of the European Physical Society, Bucharest, Romania.

Van Krevelen, D. W., 1965, Fuel, 44, 229.

Whe e lock, T., ed., $197 \overline{7}$, Coal Desulfurization, Symposium Series 64, American Chemical Society, Washington, D. C.

Whitehurst, D. D., M. Farcasiu, and T. O. Marshall, 1976, EPRI Report AF-252.

Yeager, K. E., I980, Coal Clean-up Technology in Annual Review of Energy, ed. by J. M. Hollander, vol. 5 . 


\section{F. CATALYSIS}

\section{Introduction}

The development of appropriate catalysts probably provides the greatest opportunity for exerting leverage on synthetic fuel technology. The advantages of performing the necessary reactions rapidly under " mild" conditions of temperature and pressure are obvious. What a physicist approaching the subject for the first time should also bear in mind, however, is that the key desirable attribute of a catalyst is product selectivity. To put it quite simply, one wishes to produce a desired end product such as high octane gasoline. Catalysts that allow one to obtain that end product most expeditiously, and with the least amount of less valuable products, are most desirable.

In this section, we will discuss several areas of catalysis offering interesting and technologically important research opportunities. These deal with: (1) shape and size selective zeolite catalysts, especially the synthetic zeolite ZSM-5; (2) mineral matter catalytic effects in direct liquefaction: (3) surface science related to transition metal heterogeneous catalysts for synthesis gas conversion; and briefly with both (4) catalyzed gasification of carbons and coals and (5) denitrogenation and desulfurization catalysts.

As discussed in the Introduction and in the Technology Primer of Section IV A, there are two main technological strategies for coal liquefaction that are being actively pursued at present. One is indirect liquefaction. Here, one first gasifies the coal to a mixture of $\mathrm{CO}$ and $\mathrm{H}_{2}$, and then converts this synthesis gas ("syngas") catalytically to the desired hydrocarbons. This gives rise to standard classical problems in heterogenous catalysis. The other technology is direct liquefaction where a mixture of coal, process-derived solvent, and hydrogen is "cooked" at high temperature and pressure in a reactor. Topics (1), (3), and (4) above are relevant to indirect liquefaction, and (2) and (5) are pertinent to direct liquefaction.

The traditional approach to indirect liquefaction is to synthesize the desired end products directly through Fischer-Tropsch processing using transition metal catalysts, such as iron. The problem here has been product selectivity -- especially the fact that the product distribution extends to linear hydrocarbons heavier than the $C_{5}-C_{1}$ gasoline range. An alternative approach, developed recently by the Mobil Co., is to proceed from syngas to gasoline in two stages. First is the catalytic conversion of syngas to methanol ( $\left.\mathrm{CH}_{3} \mathrm{OH}\right)$; this is an established commercial technology. Then follows catalytic conversion of methanol into high octane gasoline using a recently developed size and shape selective synthetic zeolite. Current efforts attempt to carry this type of approach even further, by combining transition metal Fischer-Tropsch catalysts (e.g., Fe, Ru) with shape-selective zeolites (e.g. ZSM-5) to effect direct conversion of synthesis gas to gasoline-range hydrocarbons (Chang et al, 1979; Huang and Haag, 1980; Rao and Gormley, 1980).

While zeolite catalysts are very widely used in the petroleum industry, being by dollar value the most important of catalysts used commercially, the unusual properties offered through their unique type of crystal structure are not well explored by physicists. Thus they combine the possiblity of novelty in physics with practical significance. Mineral matter catalytic effects [topic (2) above], while not being so evocative of novel physics, constitute a relatively new area and one where physics technique has already been usefully applied. Discussion of topic (3), the surface science related to transition metal heterogeneous catalysis for fuel synthesis, is very important technologically and interesting scientifically, and general interest in this area is already well established among solid state physics. Remarks here will highlight a few questions of technological importance which deserve special emphasis in research. Brief remarks on (4) and (5) deal with areas of established chemical interest which are relatively unknown to physicists.

\section{Zeolite size and shape selective catalysts}

Zeolites (Smith, 1976; Eberly, 1976 ; Kerr, 1981) are high surface area alumino-silicates containing exchangeable, change-balancing cations. The crystal structure gives rise to cavities and channels 
on an atomic scale, and of well defined shape. (See Fig. IV F-1 for an illustration of such a structure, that of the zeolite erionite.) The properties of zeolites that are important for catalytic applications are: (1) large surface area; (2) shape and size selectivity; (3) very strong acidity -- (By acidity we mean that the zeolites are proton donors. The availability of protons is an important factor in the practical importance of zeolites. In catalytic "cracking" a proton breaks a carbon-carbon bond, thus breaking large hydrocarbons into smaller molecules. In a commercial "cat-cracker" the hydrogen in the zeolite would be replenished by some feeding process.) --; (4) the presence of strong

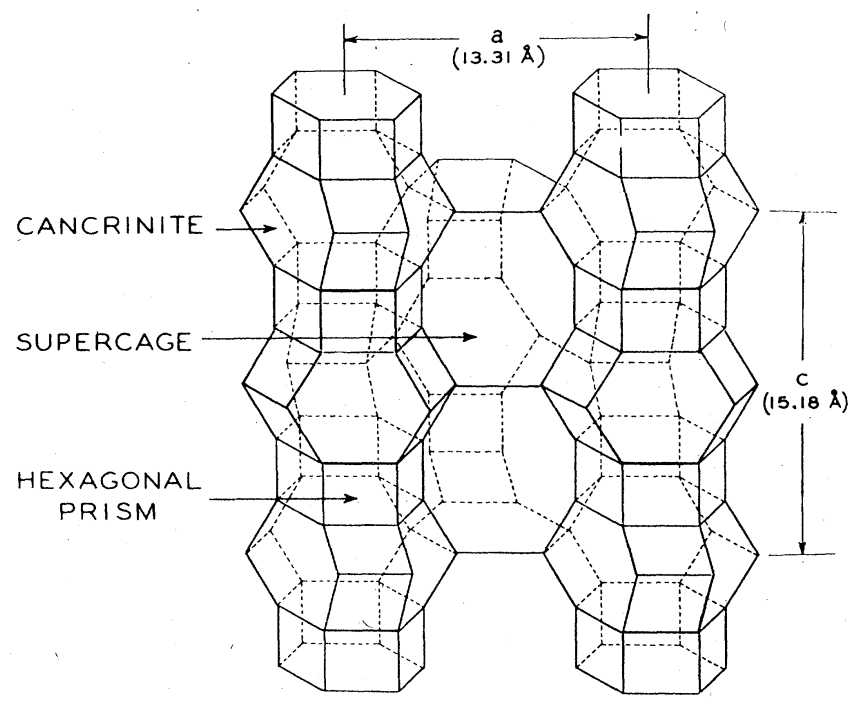

FIG. IVF-1. Line drawing of erionite. The drawing depicts the framework structure made up of cornerlinked tetrahedra in which small atoms (denoted $T$ atoms) lie at the centers, and oxygen atoms lie at the corners. The $T$-atoms in erionite are $\mathrm{Al}$ and $\mathrm{Si}$. (In synthetic zeolites, neighboring atoms in the periodic table, such as $\mathrm{Ga}, \mathrm{Ge}$, and $\mathrm{P}$, might be incorporated.) The lines in the drawing connect the $T$-atoms. The large sorption cage (super cage) in erionite is enclosed by rows consisting of alternating units of smaller cages, called cancrinite cages, and hexagonal prisms. The sorption cavities constitute a three-dimensional pore network. Each cavity has a length of $15.1 \mathrm{~A}$ and a free cross-sectional diameter of 6.3 to $6.6 \AA$. Sorbate molecules can enter the cavity through six elliptical openings (minimum and maximum diameter of 3.5 and 5.2 A respectively) formed by rings of oxygen (after T. E. Whyte, Jr., E. L. Kerr, and P. B. Venuto, J. Catalysis $\underline{20}, 88$ (1971)). internal electrostatic fields; and (5) the possibility that zeolites can be used to make dual-function catalysts by being used to support ultrafine particles of transition metals. Since the acidity affects the particular organic chemical reactions involved, physicists will probably not be very directly concerned with this aspect of zeolite behavior. On the other hand, physicists probably will be interested in gaining further understanding of the shape and size selectivity which arises from the fact that the pore structure determines the size and shape of the organic molecules that can pass through the zeolite.

The zeolite of most current interest in the context of coal utilization and synthetic fuel production is a synthetic zeolite developed by Mobil Res. and Dev. Corp., and designated ZSM-5. In a process recently developed by Mobil, ZSM-5 is used to catalyze conversion of methanol ( $\mathrm{CH}_{3} \mathrm{OH}$ ) into high octane gasoline (Derouane and Vedrine, 1980; Derouane and Gabelica, 1980). So far as the production of gasoline is concerned, the important factor is that the molecular size restrictions for diffusing through the ZSM-5 channel structure discourage the formation of undesired larger hydrocarbons, as occurs in Fischer-Tropsch processes. (ZSM-5 has two orthogonal channel systems with an intermediate sized access diameter, relative to other zeolites, of 5.5 to $6 \AA$ ).

The question here of most immediate interest to physicists probably is how to understand the dynamics of molecular diffusion through the zeolite channel structure. Data obtained by traditional bulk sorption methods may be quite misleading, since uptake behavior may be determined by phenomena other than intracrystalline diffusion (Dubinin et al, 1980). The most promising tool for achieving the necessary understanding is high power pulsed NMR (Karger and Caro, 1977).

Motion of diffusing methanol, or other organic molecules will strongly affect their NMR behavior. Nuclear interactions are averaged by molecular motions occurring on time scales shorter than $10^{-5}-10^{-6}$ seconds

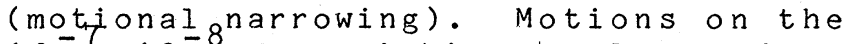
$10^{-7}-10^{-8}$ second time scale produce fluctations which cause energy exchange between the nuclei and their environment (spin-lattice relaxation). Wideline NMR measurements of the shape and width of the NMR absorption, and transient NMR measurements of 
the spin lattice relaxation time $\left(T_{1}\right)$ probe these motions. The methods of nuclear spin relaxation analysis permit determination of correlation times for molecular motion and mean interaction energies for absorbate molecules (Pfeifer, 1972). Pulsed field gradient NMR measurements permit determination of the diffusion coefficients directly (Karger and Caro, 1977; Karger et al, 1978; Karger and Volkmer, 1980).

Besides using ordinary organic molecules, it may be useful to work with deuterated ones. Resonance properties of deuterons are dominated by the interaction of the nuclear electric quadrupole moment with electric field inhomogeneities coming from their bonding in the molecule. This is a much stronger, and more local, interaction than the magnetic interactions determining the NMR behavior of protons, and thus provides complementary information on molecular motion. One can extract information on correlation times and activation energies for molecular diffusion, and their temperature variation, from analysis of the NMR linewidth behavior.

On the theoretical side, the suggestion has been made (Rabo, 1980) that in the diffusion of organic molecules through zeolites, the zeolite acts as a strong solid electrolyte, interacting strongly with the polarizable molecules. This raises the question as to whether there are useful analogies to the theory of ionic conductivity which might be developed.

As in the case of the studies on water in coal (Mraw and Silbernagel, 1981), complementary measurements of equilibrium properties, especially specific heat, may prove helpful, although the situation, involving reactions of organics, is obviously more complex. Heat capacity measurements should reveal long time, macroscopic average behavior for molecules resident in zeolites. Such measurements could provide valuable information about adsorption, if the organic fluid resident in the pores had a phase transition in its free state within the temperature range investigated (i.e. one could recognize change in the nature of the transition through the heat capacity behavior).

There is great current interest in using zeolites as supports for very small transition metal particles (Cusamono et al, 1981; Chang et al, 1979; Huang and Haag, 1980; Minachev and Isakov, 1976; Gallezot, 1979; Naccache et al, 1977 (also see some earlier studies cited in Naccache et al)). The particle can be supported on the surface or (with greater difficulty, but of more scientific -- not necessarily more technological -- interest) imbedded in the zeolite, i.e. using the cage structure to stabilize very small dispersed metal particles or clusters in the range 7-10A. The practical interest in such systems, as discussed in the introductory remarks of IVF1 above, is to gain the capability to effect the direct conversion of synthesis gas to gasoline-range hydrocarbons. It would be interesting to explore the possible difference in behavior between transition metal particles on the surface of a zeolite and particles embedded within the cages of the zeolite structure. Undertaking such studies hinges on being able to embed such particles or clusters. Work by Naccache et al (1977) for platinum catalysts shows that small particles of $8-10 \AA$ size are homogeneously dispersed inside the supercages of Linde $Y$ zeolite. Once formed, the particles are too large to escape through the small $7.2 \AA$ port windows (i.e. largest atomically defined openings from the supercage).

Study of such zeolite-metal particle systems could offer several interesting possibilities for physics work of practical import. In the making of such systems one might consider the use of solid state channeling phenomena (i.e. particularly considering the natural channel structure of the zeolite itself), such as are used in ion implantation techniques for semiconductors. One might be able to control both composition and location of metal clusters. EXAFS (extended $x-r a y$ absorption fine structure) could be applied to questions of small particle structure (interatomic distances and particle shapes). (Renouprez et al, 1980) and of metal-zeolite interactions (the particles may be significantly electron deficient) (Weber et al, 1980). EXAFS can also be applied to investigate the local coordination of cations in zeolites (Morrison et al, 1980a, $1980 \mathrm{~b})$, and can be used to investigate metal-clustering phenomena. The whole array of modern surface physics theoretical and experimental techniques for studying the electronic structure of surfaces could be used to study the charge transfer between metal and zeolite. This is important for determining the "acidity", and hence the way in which 
particular reactions occur. XPS (X-ray photoemission spectroscopy) and associated theoretical calculations of electron structure should be valuable. Study of quantum size effects by susceptibility and NMR measurements should also be useful. Besides examining the electronic structure per se, one would want to study vibrational spectra, perhaps by Raman, $x-r a y$, or neutron scattering, in order to reveal effects of the metal particles bonding to the zeolite internal surface.

\section{Mineral matter catalytic effects in direct liquefaction}

There is substantial evidence (Granoff and Montano, 1981; Montano, 1980) that the native mineral residue in coal plays an important catalytic role in direct hydroliquefaction processes. This effect is thus far neither scientifically understood nor controllable. It is important to learn which are the catalytically effective minerals, and how they work.

Certain naturally occurring minerals in coal such as pyrite $\left(\mathrm{FeS}_{2}\right)$ and iron bearing clays have been shown to enhance the liquid yield and product quality (i.e. selectivity toward desirable weight range of hydrocarbons). Similar effects have been observed when additional pulverized pyrite was deliberately added to coal, and this suggests the possibility of using inexpensive disposable catalysts for direct coal liquefaction.

In direct 1 iquefaction processes, coal, process-derived solvent, and hydrogen are fed first into a preheater, where liquefaction begins, and then into a reactor where further conversion of the initially "solubilized" coal takes place. It was found that recycling mineral matter residue enhances the yield of "distillate" products. The nature and extent of these mineral effects should be assessed as one step toward optimum conversion of coal to liquid products, and perhaps toward the development of relatively inexpensive disposable catalysts. Understanding of the role of the mineral matter is also desirable to develop direct 1 iquefaction processes that are not too narrowly restricted in regard to feed coal type.

Observation of mineral matter effects has thus far consisted primarily of noting the correlation of minerals present with product composition. The key result has been that pyrite enhances conversion, and that the effectiveness of different pyrites (i.e. the term "pyrite" is often loosely used to mean iron sulfides of varying stoichiometry and crystal structure) appear to vary considerably. Under the typical high temperature and pressure conditions of liquefaction, pyrite is partially transformed into a non-stoichiometric iron sulfide known as pyrrhotite $\left(\mathrm{Fe}_{1-\mathrm{x}} \mathrm{S}\right)$. It has been suggested that pyrrhotite is really the active catalyst for coal liquefaction. It is important, therefore, to learn the role of pyrrhotite in coal conversion.

Mossbauer spectroscopy has been introduced ( $\mathrm{Gr}$ anof $\mathrm{f}$ and Montano, 1981 ; Montano, 1980) to study iron-containing species, such as pyrrhotites, in coal liquefaction residues. In particular, the various $\mathrm{Fe}_{1-\mathrm{S}} \mathrm{S}$ compounds can be distinguished through the magnetic hyperfine splitting. (This splitting depends on the number of vacancies in the neighborhood of an iron site.) Through correlation of the Mossbauer information with liquid yields and properties, these experiments have shown that an increasing number of vacancies (i.e. greater iron deficiency in the pyrrhotite) is associated with better conversion of coal to the desired liquids.

To further define the role of pyrrhotites as catalysts, it will be valuable to monitor the pyrite to pyrrhotite transformation in situ, i.e. under actual coal liquefaction conditions. Fortunately, because it depends upon gamma rays, Mossbauer spectroscopy is well suited to observing externally the pyrrhotite transformation as it occurs in a reactor, i.e. the signal is able to penetrate the reacting medium, which would be opaque to longer wavelength radiation.

It is desirable to further study the relationship between vacancy concentration and pyrrhotite catalytic activity. Apparently the vacancies act to facilitate atomic migration. Systematic studies of catalytic activity in pyrite materials with varying vacancy content are worthwile. Recent work (Graham et al, 1981) indicates the potential value of shock-wave activation (Davison and Graham, 1979) to generate vacancies in pyrites for such studies. The starting and shock activated material were analyzed by $X-r a y$ diffraction and magnetization measurements. 
The measurements revealed the creation of crystal defects and the presence of a few percent pyrrhotite after shock activation. Ion bombardment is another technique that may prove useful for inducing defects in pyrites. It is important to develop other analytical tools, in addition to Mossbauer spectroscopy, to investigate mechanisms such as the pyrite to pyrrhotite transformation -- particularly because one may wish to investigate mineral systems based on other metals, such as nickel or molybdenum, which are not suitable for Mossbauer experiments. An attractive possiblity, that might be useful for "in situ" investigations under actual reaction conditions, would be some sort of acoustic spectrocopy, based say on magnetoelastic interaction.

\section{Surface science related to transition metal heterogeneous catalysts for gas synthesis}

Fundamental surface science studies contribute to the research base for technology development because they provide guidance in dealing with problems of practical catalysis (Somorjai, 1979). Two categories of such problems are: (1) attempts to find new catalysts, and therefore to recognize and understand electronic and crystal-structure effects involved in catalytic behavior, including metal-support interactions and metal cluster size effects on electronic behavior (always bearing in mind the importance of product selectivity); and (2) maintenance of the effectiveness of catalysts, i.e. problems of deactivation and "poisoning."

Practical transition metal catalytic systems typically consist of small particles, say 15-100 $\AA$ diameter, dispersed on high surface area supports, e.g. refractory oxides such as silica $\left(\mathrm{SiO}_{2}\right)$ or alumina $\left(\mathrm{Al}_{2} \mathrm{O}_{3}\right)$. It is difficult to apply those surface science $^{3}$ techniques involving high vacuum (i.,e. those measuring emitted electrons spectroscopically or using bombardment with a stream of particles) directly to such systems. (This is because of the state of cleanliness necessary to operate in high vacuum.) Moreover, the difficulty of direct examination of operating catalytic systems is compounded by the fact that the usual operating conditions also involve high pressure. Thus surface science studies pertinent to transition metal catalysts tend to fall into two rather disjoint categories, those on "realistic" distributed systems of supported small particles, and those on "model" systems involvjng single crystals of significant area $(\sim 1 \mathrm{~cm})^{2}$ and epitaxially grown single-crystal, or polycrystalline, films. Surface science experiments involving high vacuum play a central role in studying such model systems. Such studies on model systems usually deal with chemisorption and binding energies of reactive species and the associated steric and crystallographic behavior (locations and postures of adsorbed species). Interest in such model system work is already widespread and intense among physicists, chemists, and materials scientists. The present discussion is meant to identify a few questions that deserve special emphasis in research because of their technological implications.

The tools of high vacuum surface science are used to characterize model transition-metal catalytic systems through characterizing the surface structure and adsorbed species present, and probing the detailed electronic structure, including the mapping of the electron charge density distribution (Somorjai, 1979; Somorjai and Van Hove, 1979; Van Hove, 1980). Among these tools are low energy electron diffraction (LEED); surface-sensitive extended $X-r a y$ absorption fine structure (SEXAFS); low-, medium-, and high-energy ion scattering (LEIS, MEIS, and HEIS); high resolution electron energy loss spectroscopy (HREELS); Auger electron spectroscopy (AES); and angular resolved ultraviolet photoemission spectroscopy (ARUPS).

Surface science studies of additives to the clean metal surface (i.e. additives other than the molecular species involved in the particular reactions of interest) deserve special emphasis. Such additives play a significant role in controlling the chemistry of catalytic reactions, and may be harmful (poisons) or helpful (promoters).

Common poisons are carbonaceous deposits, sulfur, and nitrogen. These may come from impurities present in the reactants (e.g. sulfur present in coal used to make syngas) or may develop as products of side reactions (e.g. in hydrogenation of carbon monoxide on iron metal, the catalyst loses effectiveness through buildup of a carbon layer). A question of great importance, naturally, is how the poisoning occurs. Several mechanisms 
are possible, and it is often not clear in a given case which mechanism applies. Simple mechanisms for poisoning or deactivation are coverage of the active catalyst site or, in the case of small particle systems, sintering together of the particles with consequent reduction in active surface area. More interesting from the physics point of view are possibilities such as surface structural transformations or a change in the electronic states of the pertinent surface metal atoms. Questions of interest include how to understand any correlations between surface restructuring (rearrangement of surface atoms) and poisoning of catalytic activity; and does a poison work by blocking sites on a one-to-one basis, or does the poison operate via a long-range electronic interaction via the metallic substrate? The same species may be either a poison or a promoter depending on circumstance. For example, potassium acts as a poison for the hydrogenation of carbon monoxide by iron metal; but potassium improves the catalytic activity of an oxidized iron metal surface.

The way in which poisons act can be examined by a combined study of surface morphological changes during adsorption of the poison (e.g. site coverage and/or reconstruction as mentioned above) and of detailed electronic structure, i.e. electronic charge density studies. The most powerful of surface science tools to probe electronic structure is angularly resolved ultra-violet photoemission spectroscopy (ARUPS). ARUPS is valuable, of course, not only for studying detailed working of poisons but also for general probing of effects associated with chemisorption, such as surface states and adsorbate energy bands. ARUPS allows one to look directly at charge rearrangement at the surface. Such studies promise to be most powerful in conjunction with self-consistent calculations of surface electronic structure and chemisorption. Such calculations are in a very active and advancing stage of development.* These calculations, which can

* Some references pertinent to self-consistent surface electronic structure calculations are: Appelbaum and Hamann, 1978; Arlinghaus et al, 1980a, 1980b; Feibelman and Hamann, 1979; Goddard, 1981; Jepsen et al, 1978; Kasowski and Caruthers, 1980; Kleinman and Mednick, 1981; Krakauer and Cooper, 1977; Louie et al, 1976; Ma et al, 1981; Posternak et al, 1980, Wang and Freeman, 1979. yield complete charge density mappings, aid in understanding the site selection of an adsorbed species, including the important question of whether the adsorbed molecule penetrates the surface layers of the host metal.

"Model" system studies of adsorption of large organic molecules on transition metal surfaces are quite limited as yet. Few experimental studies have been done using techniques other than LEED (Somorjai and Van Hove, 1979). One would also hope to extend self-consistent surface electronic structure calculations to be able to deal with chemisorption of large molecules. Recently developed techniques for ab-initio calculations for electronic structure of large molecules (Kaufman et al, 1979) should prove valuable in this regard. Nevertheless, this is a formidable problem.

Traditional surface science studies and actual catalytic conditions (even for "model systems) are segarated by an enormous pressure disparity ( $10^{-8}$ torr compared to $10^{5}$ torr). An important recent development is having reaction chambers, i.e. small enclosures, incorporated into vacuum apparatus, in such a way that a sample can be characterized under high vacuum before reaction; then the reaction chamber can be isolated and the surface reaction carried out under "realistic" conditions; and finally the high vacuum can be restored, and the state of the sample can be examined after the reaction (Somorjai, 1979; Goodman et al, 1980). Thus the whole study is done in situ without removing the sample from the controlled atmosphere enclosure. (In the third stage of this procedure, of course, some caution in interpretation is necessary because of possible changes in chemisorbed structures caused by pumping down to very low pressure.) This technique was used recently to examine the effect of surface chemical composition on the kinetics of the catalytic methanation reaction over a single crystal $\mathrm{Ni}$ (100) catalyst (Goodman et al, 1980).

For studying electronic aspects of chemisorption of transition metals under increasing pressure of the reactive atmosphere, one should investigate the capabilities of electromagnetic (optical) reflectance spectroscopy as a substitute for electron emission spectroscopy, eliminating the need for high vacuum. It is true that reflectance spectroscopy is not usually regarded as surface effect selective. Perhaps a grazing angle technique could be used to 
overcome this. Careful feasibility estimates for a range of spectral regions would seem to be desirable.

In addition to the work on model crystal systems, crystal-structure and electronic-structure work on the dispersed metal particle systems that are used in practice is called for. Sinfelt (1969, 1977) has explored the properties of bimetallic cluster catalysts. Such a catalyst system, consisting of very small metal clusters dispersed on the surface of a carrier such as silica or alumina, is made by impregnating the carrier with an aqueous solution of salts of the two metals, drying, and then reducing the salts in a stream of hydrogen at elevated temperatures. One obtains mixed clusters of two metals even for cases, such as $\mathrm{Ru}-\mathrm{Cu}$ or Os-Cu, which show very low miscibility in the bulk. Moreover, the properties are markedly affected, on going to very small particles, by the tendency for surface segregation, e.g. the surface becoming very $\mathrm{Cu} r i c h$ in the $\mathrm{Cu}-\mathrm{Ru}$ system leading to a situation with a layer of copper on a core of ruthenium. Such catalysts offer improved selectivity for certain reactions. A start has been made on using EXAFS to examine the structure and composition of such systems (Sinfelt, 1977). (Note that this is very similar to the use, suggested above, of EXAFS to investigate metal particles inbedded in the zeolite ZSM-S.) It should be possible to carry out self-consistent calculations of the electronic structure of such systems (Ma et al, 1981).

Systems that are of interest in modeling bimetallic cluster catalysts are epitaxially grown films, where one deposits a monolayer, or a few layers, of one transition or noble metal on top of another metal (Biberian and Somorjai, 1979; Richter et al, 1981; Ma et al, 1981). Such epitaxial deposition might even be relevant to practical catalysts, if the deposition is on an appropriate support substrate (e.g., if substrate for bimetallic film structure is a transition metal oxide).

Morphological changes of small metal particle catalyst systems in reactive atmospheres have recently been studied using modern surface science techniques (Schmidt, 1981). With STEM (scanning transmission electron microscopy) and XPS, the behavior of Pd-Pt alloy particles of 20 to $200 \AA$ dimension was observed. Sintering in an oxygen atmosphere, and the reduction back to metal particles in a hydrogen atmosphere were observed; and cracking processes to get smaller particles and reannealing to get back to larger particles could be followed.

Metal-support interactions and the phenomena, already mentioned, of surface segregation (Brundle and Wandelt, 1981; Abraham, 1981), are important questions in the use of dispersed-particle transition-metal alloy catalyst systems. Consideration of interfacial energy should relate to the curvature (wetting angle) of small metal particles sitting on a support. Formation of cracks in such particles, again should be understood on the basis of interfacial effects. XPS and SIMS (secondary ion mass spectroscopy) are among techniques that have been used to study surface segregation in alloy systems (Brundle and Wandelt, 1981). The variation of this segregation with reactive atmospheres is a particularly interesting feature. Theory should be extended to cover this.

Work has recently been reported indicating the possible importance of metallic glasses, made by splat cooling, as catalytic systems (Smith et al, 1981). Pd/Si glasses, made by splat cooling, are active hydrogenation catalyst systems. During the hydrogenation with deuterium of cis-cyclododecene these systems produce less trans insomerization, more dideuto-saturate and less extensive exchange than crystalline Pd. Such study of amorphous catalysts must be regarded as being at an early exploratory stage, but the potential scientific and practical interest warrants encouragement of further work of this type.

In closing this subsection on surface science related to transition metal heterogeneous catalysis, we wish to emphasize the value of self-consistent surface electronic structure calculations, in conjunction with experiment, for understanding such important aspects of chemisorption as locations, binding energies, and postures of adsorbed species. Such calculations, employing powerful iterative computational techniques, allow one to obtain a realistic picture of the complex charge distribution pertinent to the chemisorption involved in catalysis. These calculations are also pertinent to understanding the key question of whether chemisorption of a molecule of interest (e.g. $\mathrm{CO}$ or $\mathrm{H}_{2}$ ) on a specific surface of a particular material will be dissociative, yielding atoms in a state suitable for 
reactions yielding the desired hydrocarbons. Conversely, these calculations can give guidance to understanding detrimental "poisoning" effects, e.g., the binding energy of an atom such as sulfur may allow it to preferentially occupy a site that otherwise would be the preferred site for chemisorption yielding desired intermediate species for catalytic synthesis. Incorporation into such calculations of methods for simulating the effects of small amounts of additives (promoters) that enhance catalytic effectiveness, would be highly desirable.

\section{Catalyzed gasification of carbons and coals}

It has been known for many years that small amounts of a wide variety of inorganic impurities can effectively catalyze the reactions of carbon with gases such as oxygen, carbon dioxide, steam, and hydrogen (McKee, 1981). Extension of such studies to coal itself is important. The most practical catalysts for catalytic direct methanation of coal are salts, e.g., carbonates, of alkali metals and alkaline earths. Understanding of temperature effects is important. The catalytic gasification goes up strongly on approaching the melting of the salt, and one would desire an effective catalyst at lower temperatures. (Methanation is exothermic and hence thermodynamically most efficient at low temperature.) Spectroscopic experiments should be developed to study the chemical state of the catalytic species. One possibility is ${ }^{2} \mathrm{Na}$ nuclear quadrupole resonance, but practical problems arising from distributions in the field gradients would make detection and analysis difficult; moreover, the interpretation, given the absence of knowledge of the coordinating atoms, would be hazardous. NMR and EXAFS might be better probes for this purpose. Measurements pertinent to migration of the catalyst particles through the pore structure of coal also are important.

A striking feature, apparently central to the mechanism of catalytic gasification in model experiments on graphite is the cutting of channels by catalytic microparticles (e.g., see McKee, 1981). Baker (1979) has developed a controlled-atmosphere transmission electron microscope (CAEM) technique enabling him to film the channel cutting process as it occurs.
This technique promises to be particularly valuable in identifying the crucial steps in thermal activation of catalytic gasification.

\section{Denitrogenation and desulfurization catalysts}

Desulfurization and denitrogenization reactions, which require appropriate catalytis, are an important step in the production of liquid fuels from coal (Grange, 1981). The catalysts that appear most effective for these reactions are molybdenum/cobalt/nickel oxides in slurries. Modern physics techniques might play a significant role in the development of highly selective catalysts, by providing information about the surface/solid state properties responsible for the catalytic effectivenss of these materials. Such properties include oxidation states of the transition metals, their distribution, crystal fields in the support material, and mechanical integrity after long term use in a hostile environment.

\section{Recommendations}

1. It is important to understand the dynamics of the diffusion process through the zeolite channel structure. -- Wideline and transient NMR, including pulsed magnetic field gradient studies, are pertinent. Isotopic experiments with deuterated molecular species could provide additional information.

2. We strongly recommend making and studying systems having very small transition metal particles or clusters embedded in zeolites. Both experimental and theoretical studies on such systems should prove valuable.

3. Work should be done to systematically study the role of vacancies in the catalytic activity of pyritic materials. In such studies, shock wave activation or ion bombardment may be useful for creating vacancies in a controlled way.

4. It is important to develop analytical tools other than Mossbauer spectroscopy, to investigate changes 
in mineral matter, such as the pyrite to pyrrhotite transformation, under actual operating conditions. Acoustic probes should be considered.

5. An important problem in catalysis is to understand the way in which "poisons" act. For this purpose, surface science studies, on "model" systems (single crystals. epitaxially grown films) should be useful. Examination of surface morphological changes, and of detailed electronic structure using angularly resolved ultra-violet photoemission spectroscopy (ARUPS) and associated self-consistent calculations of the electronic structure, should be valuable in this regard.

6. Work should be done on further development of technique for "in situ" examination of "model" systems, involving both high vacuum characterization of the sample and the carrying out of reactions of interest, without removing the sample from the controlled atmosphere enclosure. The possible advantages (and disadvantages) of electromagnetic reflectance spectroscopy, possible at higher pressures, should be considered.

7. There is interesting and useful research to be done to gain further understanding of bimetallic catalyst systems, including work on epitaxially grown "model" film systems. Such "model" bimetallic film systems offer much opportunity for productive theoretical work involving self-consistent surface electronic structure calculations. Effects of particular interest for dispersed supported small particle transition metal alloy systems are metal-support interactions and the phenomenon of surface segregation of components.

\section{References}

Abraham, F. F., 1981, Proceedings of the $27 t h$ National Symposium of the American Vacuum Society, 1980, Journal of Vacuum
Science and Technology, March-April 1981. Appelbaum, J. A. and D. R. Hamann, 1978, Solid State Commun . 27, 881 .

Arlinghaus, F. J., J. G. Gay, and J. R. Smith, 1980a, in Theory of Chemisorption, edited by J. R. Smith, Springer-Verlag, New York.

Arlinghaus, F. J., J. G. Gay, and J. R. Smith, 1980b, Phys. Rev. B 21, 2055.

Baker, R. T. K., 1979, Catal. Rev. - Sci. Eng. 19, 161 .

Biberian, J. P., and G. A. Somorjai, 1979, J. Vac. Sci. Technol. 16, 1073.

Brundle, C. R., and K. Wandelt, 1981 , Proceedings of the $27 \mathrm{th}$ National Symposium of the American Vacuum Society, 1980, Journal of Vacuum Science and Technology, March-April 1981.

Chang, C. D., W. H. Lang, and A. J. Silvestri, 1979, J. Catal. 56, 268.

Cusamano, J. A., D. L. King, and R. L. Garten, 1981, to appear in Catalysis Reviews - Science and Engineering, Vol. 21 .

Davision, L., and R. A. Graham, 1979, Physics Reports 55, 255.

Derouane, E. G., and J. C. Vedrine, 1980a, J. Molec. Catal. 8, 479.

Derouane, E. G., and Z. Gabelica, 1980b, J. Catal. 65, 486.

Dubinin, M. M., V. A. Gorlov, and A. M. Voloshchuk, 1980, in Proceedings of the Fifth International Conference on Zeolites, edited by L. V. Rees, Heyden and Son, London.

Eberly, P. E., Jr., 1976, in Zeolite Chemistry and Catalysis, edited by J. A. Rabo, ACS Monography 171, American Chemical Society, Washington, D.C., pp. 392-436.

Feibelman, P. J. and D. R. Hamann, 1979, Solid State Commun. 31, 413.

Gallezot, P., 1979, Catal. Rev. Sci. Eng. 20,121 .

Goddard, W. A. III, 1981, Proceedings of the 27 th National Symposium of the American Vacuum Society, 1980, Journal of Vacuum Science and Technology, March-April, 1981 .

Goodman, D. W., R. D. Kelley, T. E. Madey, and J. T. Yates, Jr., 1980, J. of Catalysis 63, 226.

Graham, R. A., B. Morosin, P. M. Richards, F. V. Stohl, and B. Granoff, 1981, in Chemistry and Physics of Coal Utilization -1980, edited by B. R. Cooper and L. 
Petrakis, AIP Conference Proceedings No. 70, AIP, New York.

Grange, P., 1981, Catal. Rev. 21, 135.

Granoff, B., and P. A. Montano, 1981, in Chemistry and Physics of Coal Utilization - 1980, edited by B. R. Cooper and L. Petrakis, AIP Conference Proceedings No. 70, AIP, New York.

Huang, T. J. and W. O Haag, 1980, presented at The Second Chemical Congress of the North American Continent, Las Vegas, August, 1980. (Cited in Chemical and Engineering News, Sept. 8, 1980, p. 44).

Jepsen, O., J. Madsen, and 0. K. Andersen, 1978, Phys. Rev. B. 18, 605.

Karger, J., and J. Carro, 1977, J. C. S. Faraday, Trans. I, 731363.

Karger, J., M. Bulow, P. Struve, M. Kocirik, and Z. Zikanova, 1978, J. C. S. Faraday Trans. I 74, 1210 .

Karger, J., and P. Volkmer, 1980, J. C. S. Faraday Trans. I, 76, 1562.

Kasowski, R. V., and E. Caruthers, 1980 , Phys. Rev. B 21, 3200.

Kaufman, , J. J., H. E. Popkie, and P. C. Hariharan, 1979, pp. 415-435 in Computer Assisted Drug Design, edited by E. C. Olson and R. E. Christoffen, ACS Symposium Series 112, Amer. Chem. Soc., Washington, D.C.

Kerr, G. T., 1981, to appear in Catalysis Reviews - Science and Engineering, Vol. 23.

Kleinman, L. and K. Mednick, 1981, Phys. Rev. B. 23, 4960.

Krakauer, H. and B. R. Cooper, 1977, Phys. Rev. B 16, 605.

Louie, S. G., K. M. Ho, J. R. Chelikowski, and M. L. Cohen, 1976, Phys. Rev. Lett. 37, 1289 .

$\mathrm{Ma}$, C. Q., H. Krakauer, B. R. Cooper, and I. Kulikov, 1981, Proceedings of the 27th National Symposium of the American Vacuum Society, 1980, Journal of Vacuum Science and Technology, March-April, 1981.

McKee, D. W., 1981, in Chemistry and Physics of Coal Utilization - 1980, edited by B. R. Cooper and L. Petrakis, AIP Conference Proceedings No. 70, AIP, New York.

Minachev, Kh. M. and Ya. I. Isakov, 1976, in Zeolite Chemistry and Catalysis, edited by J. A. Rabo, American Chemical Society, Washington, D.C. (ACS Monography 171).

Montano, P. A., 1980, to be published in Advances in Chemistry, "Recent Chemical Applications of Mossbauer Spectroscopy," edited by J. Stevens and G. K. Shenoy.

Morrison, T. I., A. H. Reis, Jr., E. Gerbert, L. E. Iton, G. D. Stucky, and S. L. Suib, 1980a, J. Chem. Phys. 72, 6276.

Morrison, T. I., L. E. Iton, G. K. Shenoy, G. D. Stucky, S. L. Suib, and A. H. Reis, Jr., 1980b, J. Chem. Phys. 73, 4705.

Mraw, S. C., and B. G. Silbernagel, 1981, in Chemistry and Physics of Coal Utilization - 1980, edited by B. R. Cooper and L. Petrakis, AIP Conference Proceedings No. 70, AIP, New York.

Naccache, C., N. Kaufheer, M. Dafaux, J. Bandiera, and B. Imelik, 1977, in Molecular Sieves-II, edited by J. R. Katzer, American Chemical Society Symposium Series, pp. 538-548.

Pfeifer, H., 1972, in NMR-Basic Principles and Progress, Vol. 7, edited by P. Diehl, E. Fluck, and R. Kosfeld, Springer-Verlag, Berlin.

Posternak, M., H. Krakauer, A. J. Freeman, and D. D. Koelling, 1980, Phys. Rev. B 21, 5601 .

Rabo, J. A., 1981, to appear in Catalysis Reviews - Science and Engineering, Vol. 23 .

Rao, V. U. S., and R. J. Gormley, 1980, Hydrocarbon Processing, November, 1980.

Renouprez, A., P. 'Fouilloux, and B. Moraweck, 1980, in Growth and Properties of Metal Clusters, edited by J. Bourdon, Elsevier, Amsterdam.

Richter, L., S. Bader, and M. Brodsky, 1981, Proceedings of the $27 \mathrm{th}$ National Symposium of the American Vacuum Society, 1980, Journal of Vacuum Science and Technology, March-April, 1981.

Schmidt, L. D., 1981, Proceedings of the $27 \mathrm{th}$ National Symposium of the American Vacuum Society, 1980, Journal of Vacuum Science and Technology, March-April 1981.

Sinfelt, J. H., 1969, Rev. Mod. Phys. 5l, 569.

Sinfelt, J. H., 1977, Science 195, 641.

Smith, G. V., W. E. Brower, M. S. Matyjaszczyk, and T. L. Pettit, 1981, to appear in the Proceedings of the $7 \mathrm{th}$ International Congress on Catalysis, July, 1980.

Smith, J. V., 1976, in Zeolite Chemistry and Catalysis, edited by J.A. Rabo, ACS Monograph 171, published by the American Chemical Society, Washington, D.C., pp. $1-79$.

Somorjai, G. A., 1979, Surface Science 89, 
496.

Somor jai, G. A., and M. A. Van Hove, 1979, Adsorbed Monolayers on Solid Surfaces, Volume 38 of the Structure and Bonding Series, Springer-Verlag.

Van Hove, M. A., 1981, Proceedings of the 27 th National Symposium of the American Vacuum Society, 1980, Journal of Vacuum
Science and Technology, March-April, 1981.

Wang, C. S. and A. J. Freeman, 1979, Phys. Rev. B. 19, 793.

Weber, R. S., M. Boudart, and P. Gallezot, 1980, in Growth and Properties of Metal Clusters, edited by J. Bourdon, Elsevier, Amsterdam. 


\section{APPENDIX I. $\mathrm{CO}_{2}$ EMISSIONS: COMPARISONS OF THE USE OF COAL FOR ENERGY PRODUCTION BY VARIOUS ROUTES}

\section{Introduction}

In this appendix, we compare various coal utilization methods as sources of carbon dioxide. We delineate, where possible, how physics research could elucidate or affect this aspect of the state-of-the-art of coal utilization.

In the analysis which follows, it has been necessary to use reports and other published data that refer to specific commercial (or proposed commercial) processes. However, no comparison is intended inter se -in the first instance the relative degree of commercialization of these proposed processes are not equivalent; therefore the flow sheets will be subject to change. It is further to be emphasized that the plant efficiency (and hence its inherent $\mathrm{CO}_{2}$ production) is subject to each specific flow sheet modification; even within a given-named process significant differences can exist. Because of this sensitivity to engineering detail, differences in carbon utilization derived in this appendix indicate only general trends. We indicate an approach to this subject, and extract general conclusions relating to efficiency rather than conclusions specific to particular utilization schemes.

A second fundamental problem which occurs is the fact that the fuel value of the products of coal use are not equal by several measures, thermodynamic but also non-technical -- e.g., the societal values of electricity vs. natural gas or synthetic gasoline. In this appendix, we have disregarded what many have rightly considered important in energy analysis.

We restrict our discussion to quantitative assessments of the $\mathrm{CO}_{2}$ outputs of the technologies considered. We do not broach the subject of possible effects of $\overline{\mathrm{CO}_{2}}$ from any sources. This latter problem has received $\overline{\text { much }}$ recent attention (e.g., MacDonald, et al, 1979, 1980; Charney, et al, 1979; AAAS 1980; Council on Environmental Quality, 1981; and Abarbanel et al, 1980). Our discussion is relevant to an assessment of the trade-offs and penalties associated with various fuel strategies which could be adopted; and should be viewed as providing a method for quantitative analysis of this one limited aspect, with the primary objective of delineating the physical basis, and research avenues, pertinent to $\mathrm{CO}_{2}$ release.

To these ends, we first consider the generation of electricity using various fossil fuels, and define an index appropriate to a comparison of these fuels as sources of $\mathrm{CO}_{2}$, including the effects of such constraints as scrubbers, etc. We proceed to an analysis of $\mathrm{CO}_{2}$ emission from synthetic fuel production. This leads naturally to definition of a carbon efficiency index, and to a comparison of $\mathrm{CO}_{2}$ release associated with various ways of using the same primary resource fuel (i.e. coal). The analysis leads to some conclusions about relevant research directions:

First, the incorporation of source carbon into a synthetic fuel product (i.e., the desirable complement of $\mathrm{CO}_{2}$ effluent) increases directly as the thermal efficiency of a process for fuel synthesis increases. Thus research directed toward improving the thermal efficiency of synthetic fuel processes will have the concomitant effect of reducing the amount of $\mathrm{CO}$ released during production of the synthetic fuel. second, it is possible to substantially reduce $\mathrm{CO}_{2}$ emissions from synthetic fuel plants by providing a non-fossil source of process energy. This reduction can amount to greater than $75 \%$ of the $\mathrm{CO}_{2}$ released during conventional processing. Thus, insofar as $\mathrm{CO}_{2}$ can be reduced either by improvements in thermal efficiency or by use of non-fossil process energy, interested members of the physics community have a number of alternatives and possibilities for research opportunities in this area.

We also corroborate previous estimates used in the $\mathrm{CO}_{2}$-effects studies, that conversion of coal to a synthetic fuel and utilizing that fuel, gives a net $\mathrm{CO}_{2}$ production of about twice that for the same energy output using a comparable natural fossil fuel.

\section{$\mathrm{CO}_{2}$ evolution from direct conversion of fossil fuel to electricity}

It is convenient to define a criterion to compare efficiencies of different electric 
power plants on a carbon-evolved basis. A useful generalization can be made of a criterion used by MacDonald et al, (1980), i.e., carbon dioxide evolved in ratio to delivered energy.

If $\mathrm{w}^{c}$ is the weight $\%$ of carbon in the coal then a suitable index of performance is defined by:

$$
I=\frac{w^{c}}{\eta_{e}^{\Delta H_{C L}}}=\frac{\mathrm{kgC}\left(\text { released as } \mathrm{CO}_{2}\right)}{k W_{e^{-h r} \text { of electricity produced }}}
$$

where $\eta$ is the first law efficiency (electricity produced/combustion heat in coal), and $\Delta \mathrm{H}_{\mathrm{CI}}$ is the enthalpy of combustion per unit mass of coal.

The utility of this index is that it compares the evolution of $\mathrm{CO}_{2}$ with the useful product, i.e. units of electrical energy. There are very reliable estimates of power

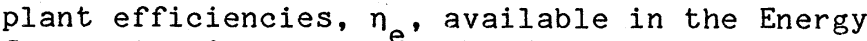
Conversion Alternatives Study (Corman and Fox, 1976). A selection of cycles of interest include conventional steam plant, conventional steam plant with scrubbers, atmospheric fluidized bed (AFB), pressurized fluidized bed $(P F B)$, integrated gasification combined cycle (IGCC), and open cycle magneto-hydrodynamic generator (MHD).
Figure $\mathrm{Cl}$ is a composite figure showing the index I as a function of power plant efficiency $n$ for a variety of fossil fuels. Anthracite is not usually considered an appropriate fuel for a power plant system and is shown only for comparative purposes. In any case, it is the least promising fuel on a carbon-evolved basis. While lignite is a slightly better fuel on this basis, its low carbon content does not, in fact, compensate for its low heating value. Furthermore, the net heating with lignite is diluted by its high moisture content.

There are two deleterious effects which have been accounted for in the calculations summarized in Figure $\mathrm{Cl}:$ a) The need to supply the latent heat of evaporation of the contained water. b) The reduction in boiler efficiency due to the increase in exhaust stack partial pressure of water vapor. Effect (a) is accounted for by reducing the heating value of the fuel accordingly; effect (b), by reducing the boiler efficiency progressively from $88 \%$ to $81 \%$ on going from bituminous coal to natural gas (Brown, 1980), since stoichiometric considerations demand an increase in the exhaust water vapor content.

Figure $\mathrm{Cl}$ shows that high volatile bituminous coal, sub-bituminous coal or residual oil behave very similarly on a (carbon

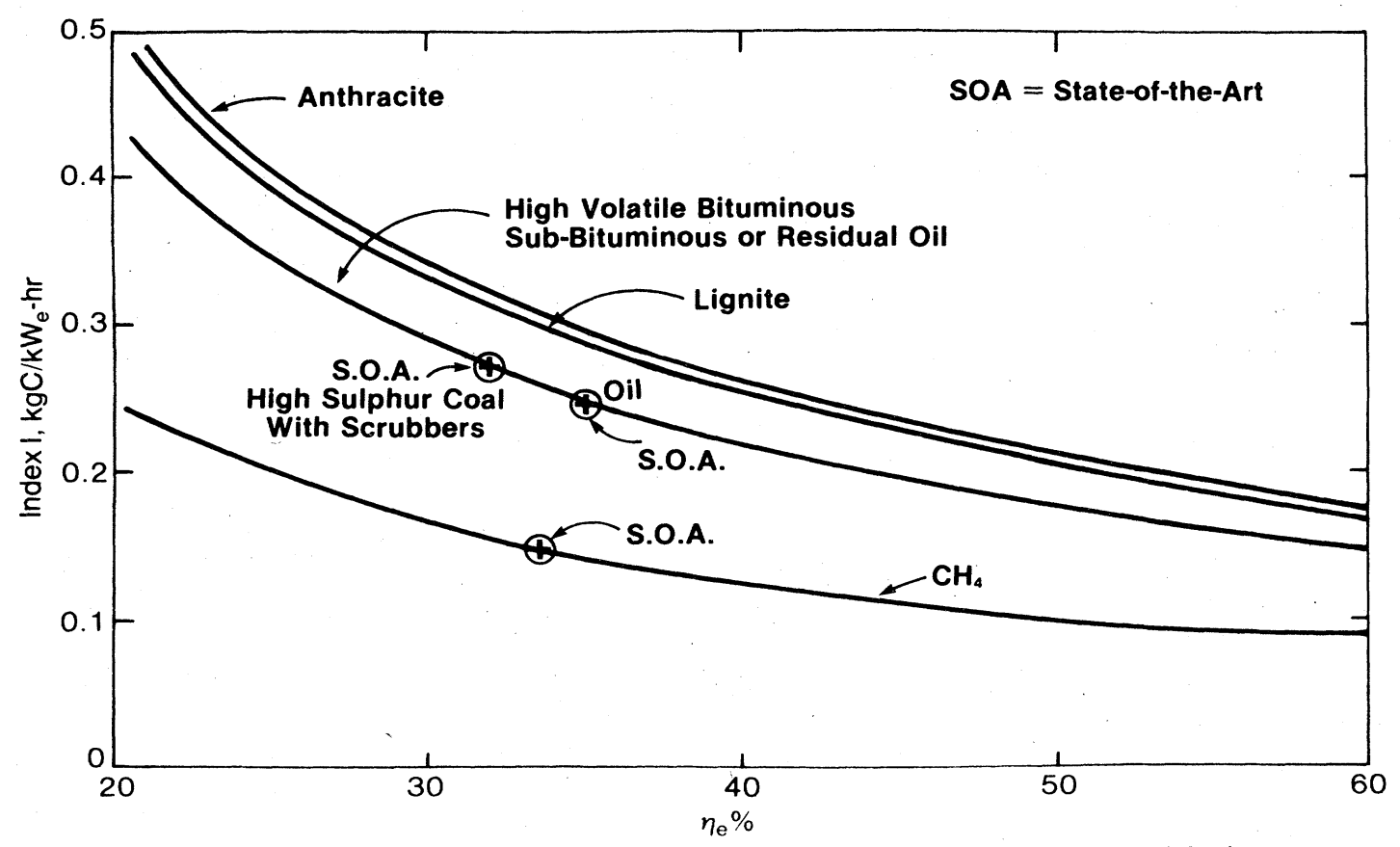

FIG. C1. Relative carbon release rates for several power plants using selected fossil fuels. 


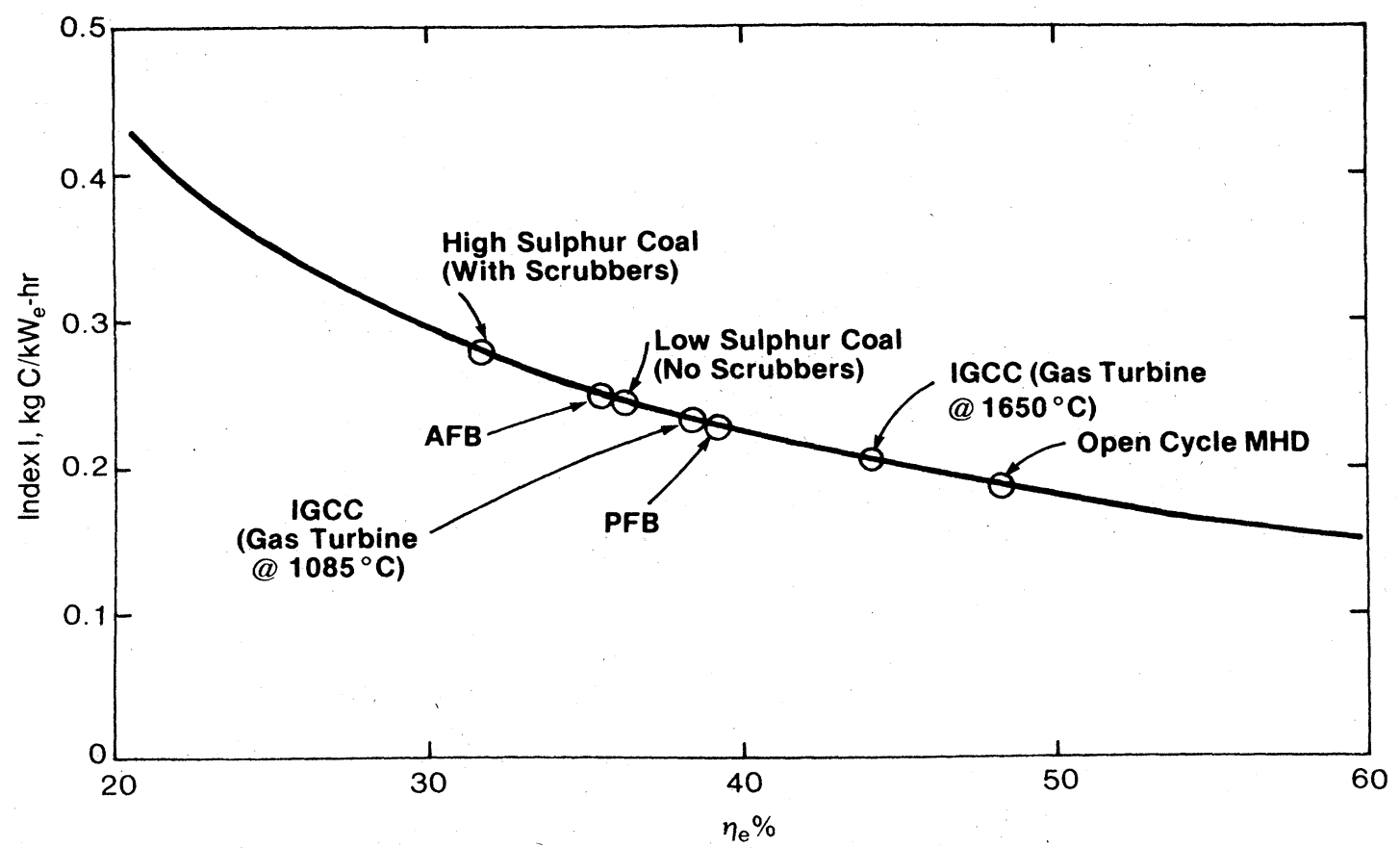

FIG. C2. Relative carbon release rates for advanced bituminous coal power plants.

evolved)/(output power) basis. Shown on this curve are the state-of-the-art (SOA) data points for an oil-fired generating plant and a high-sulfur coal powered generating plant with $\mathrm{SO}_{2}$ scrubbers.*

The lowest curve is that for a natural gas plant, where its SOA efficiency point is plotted. There is a decrease of net CO evolved by a factor of approximately 2 between the fuels giving the extremes. For example, at $35 \%$ power plant efficiency, anthracite yields $0.285 \mathrm{kgC} / \mathrm{kW}-\mathrm{hr}$, lignite 0.28 , bituminous and sub-bituminous coal 0.25 and natural gas 0.14 $\mathrm{kgC} / \mathrm{kW}_{\mathrm{e}}-\mathrm{hr}$. Reality does, however, reduce the actual power plant efficiency as the hydrogen/water content increases for the reasons previously noted, so that an assumed fixed power plant efficiency is a simplification.

Figure C2 summarizes expected values of the power plant efficiency for those plants considered by Corman and Fox, (1976), and those for which updated data are available (Pomeroy et al., 1980; Shah et al., 1980). These data all refer to the same fuel -bituminous (or sub-bituminous) coal as was used in the calculations of Figure $\mathrm{Cl}$.

\section{$\mathrm{CO}_{2}$ Evolution from direct conversion of fossile fuel to electricity}

It is significantly more difficult to develop simple indices of performance for synthetic fuel plants than for electrical generating plants. The reason is that the former produce a plethora of products whose thermodynamic worth is obscure. For example, methane is a by-product of many liquefaction schemes; is its fuel value to be included in the products, or is it a waste material? Furthermore, what is its "fuel value"? There are several candidates. The higher heating

* SO scrubbers "consume" coal by i) the need to calcine limestone or dolomite with coal heat - e.g., $\mathrm{CaCO}_{3}=\mathrm{CaO}+\mathrm{CO}_{2}$, where the lime $(\mathrm{CaO})$ is used as the sulfur absorbant, and ii) the requirement of internally consumed electricity for the gas handling equipment in the scrubber. A net $\mathrm{CO}_{2}$ evolution of 2-6 moles may be produced per mole $\mathrm{SO}_{2}$ scrubbed depending on the sulfur content of the original coal. 
values (HHV*) and lower heating values (LHV**) can be used to define First Law efficiencies:

$$
\begin{aligned}
& n^{H H V}=\frac{\text { HHV of products }}{\text { HHV of input coal }} \\
& n^{\text {LHV }}=\frac{\text { LHV of products }}{\text { LHV of input coal }}
\end{aligned}
$$

Such definitions can be very misleading if there is an input of auxiliary fuel or an export of work from the process. A further efficiency which is needed is a carbon efficiency here defined as

$$
n^{C}=\frac{C \text { in products }}{C \text { in input coal }}
$$

in which the products may be further subdivided into different useful classes, e.g., gaseous and liquid, to account for the co-generacy in many processes.

There are several classes of conversion processes to consider. These are listed with some specific examples of each in Table Cl. With each acronym the proprietary name is given for the developer of each process. These are but a few of those that can be found in the literature. Combinations are possible as shown in Table $\mathrm{C} 2$. In these data

(Harrison, 1980; Shih, 1981), the product yields (on a lower heating value basis, i.e, $n$ expressed in $\%$ ) are given for a range of products in order of increasing molecular weight, viscosity, density, etc. For example, a generic Fischer-Tropsch process using British Gas Corporation Slagger gasifiers would have 14\% LPG and 17\% gasoline (by energy content). Note SRC-I primarily makes what its name implies, solvent refined coal, $49.5 \%$ to $62 \%$ of its input coal energy. The more recent SRC-II has a lighter spectrum of products with its emphasis on fuel oil. Likewise, two modes are quoted for $\mathrm{H}-\mathrm{Coal}$ with different product spectra. The mechanism for these differences is in the recycle of hydrogen from carbonaceous residue or coal. Further differences in each process are due to

* Including latent heat of condensation. **Excluding latent heat of condensation. slightly different flow sheets, emphasizing different products.

It is important to realize the great significance of gasification of coal (to produce $\mathrm{H}_{2}$ ) even in liquefaction of coal. The quantity of $\mathrm{H}_{2}$ recycled (or equivalently a hydrogenated donor solvent), the makeup $\mathrm{H}_{2}$, and the loss of $\mathrm{H}_{2}$ by heteroatom capture hefp determine the product distribution. While undigested coal residue can be burned, it is probably optimum to gasify it as a further option, but the use of this residue obscures the specification of conversion efficiency and product spectra. Having noted all these ambiguities, the bottom line message of Tabte C2 is that the conversion efficiency on a $\eta^{\text {Lt }}$ basis ranges from $34 \%$ to $74 \%$ for a range of processes.

Is there any rational basis for a standard against which these processes (ranging from laboratory to commercial) can be compared? In the absence of a complete thermodynamic treatment, Wei (1980) has shown how simple considerations based on $\mathrm{C}-\mathrm{H}-\mathrm{O}$ stoichiometry can lead to limits on conversion efficiency. He has considered the theoretical liquefaction of graphite to 1-octene by four different processes. With the restrictions imposed by the stoichiometry conditions, all four processes lead to essentially the same maximum utilization of carbon in the liquid product, viz 50-60\%, the remainder being vented as $\mathrm{CO}_{2}$. Of course, coal is not graphite, real processes are not ideal, and 1 -octene is not the sole product in actual liquefaction. Nevertheless, the utilization of carbon in the graphite to l-octene conversion provides a simple bound which can be used as a zeroth order yardstick on carbon efficiency.

Substantial data do exist in the report literature (e.g. McNamee et al. 1978 ; Schreiner et al. 1978; and Shinnar et al. 1978); these particular references are selected for their pertinence to the present study. In particular, for example, McNamee et al (1978) provide process description of theoretical 50,000 barrel/day coal refineries producing a variety of products. Two of these processes are labeled as "catalytic hydroliquefaction" and "solvent refined coal;" these processes are herein interpreted as a generic $\mathrm{H}$-coal-type and a generic SRC process. The flow sheets in each of the quoted references, and indeed for all chemical process plants, are rather specific. These flow sheets reflect, in general, a plant 


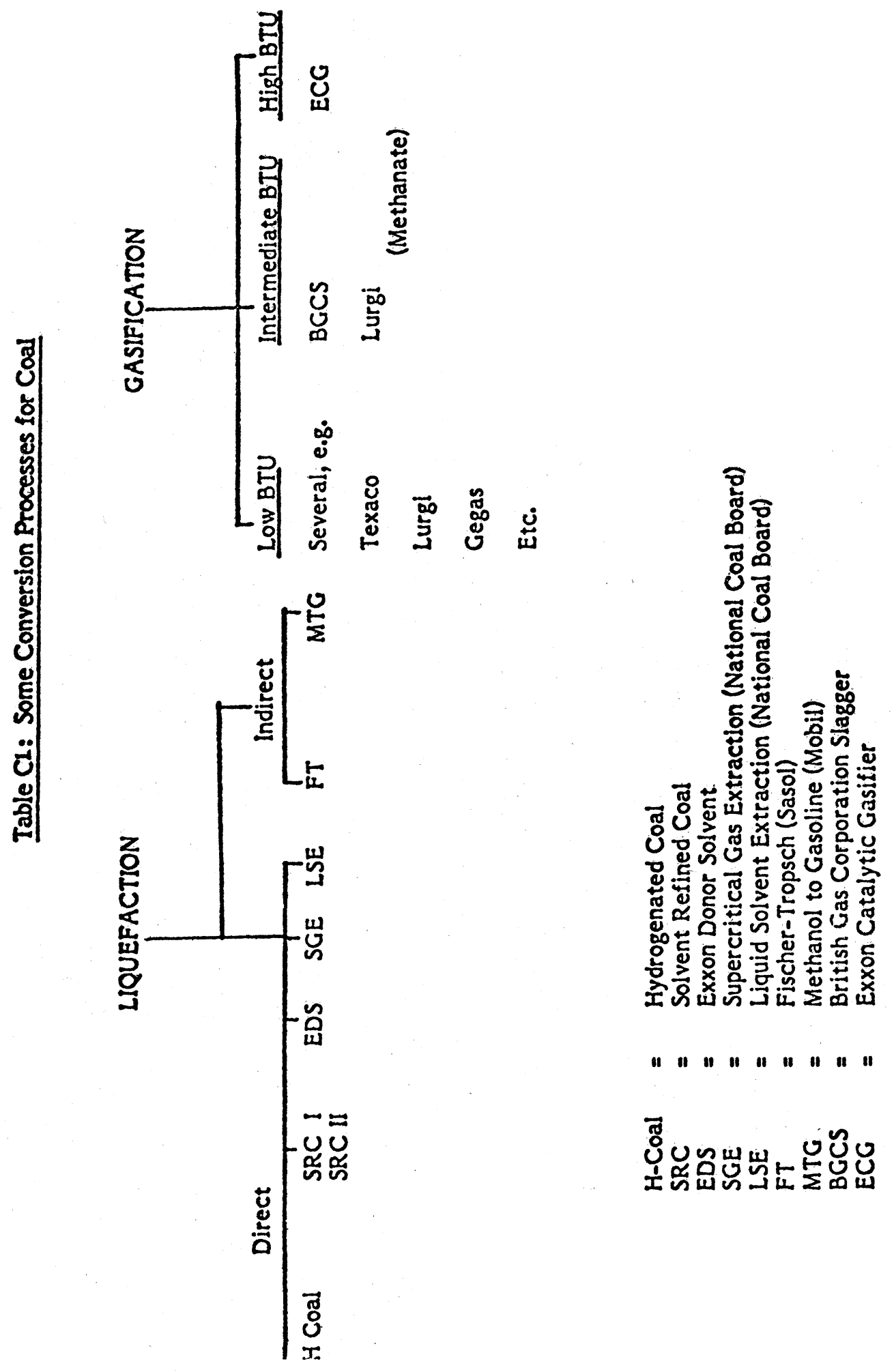




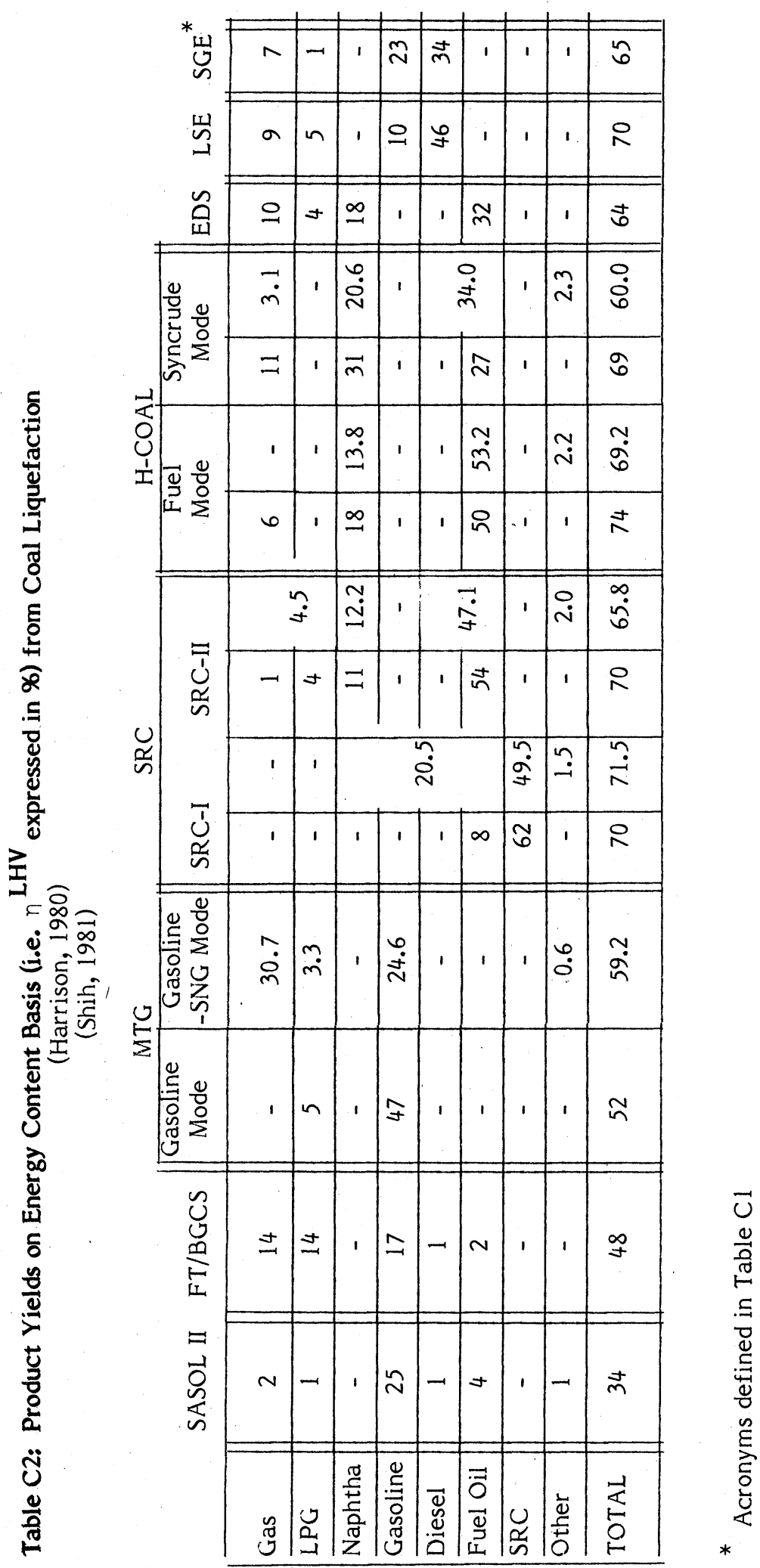

Rev. Mod. Phys., Vol. 53, No. 4, Part II, October 1981 
optimized in some way subject to inherent and/or applied constraints such as economics would impose. The pertinent conclusion is that under a different range of constraints a $r$ ather different flow sheet could emerge, and as one possible consequence, the thermal and carbon balances of the process would be changed. The reader is, therefore, cautioned. not to draw canonical conclusions with respect to any of the named processes - these are demonstratively subject, at least, to revision for engineering improvements as they near implementation.

These liquefaction processes produce naphtha, fuel oil and residual fuel as condensed phase products, plus a fuel gas, mainly hydrogen with hydrocarbon impurities $C_{4}$ or less. Of these products, naphtha is gasoline like, fuel oil can be used for purposes from running diesels to space heating (depending on further treatment), and residual fuel (or residuum) boils above $400 \mathrm{C}$. Residual fuel is used exclusively in heavy utility operations and, except where a clean coal based substitute is required, is the least desirable of the products. In the generic SRC process, residual fuel amounts to $86.5 \mathrm{wt} . \%$ of the condensed phase products; likewise, in the generic catalytic hydroliquefaction process, it amounts to 67.2 wt.\% of the condensed phase product. For this reason, these generic processes show a high thermal efficiency and a high carbon efficiency. The efficiency values are certainly too high for a working $\mathrm{H}-\mathrm{coal}$ or SRC-II plant producing "light" products.

The efficiency data for the generic catalytic hydroliquefaction and Solvent Refined Coal processes are shown in Figure $\mathrm{C}_{3}$ as two sets of adjacent bars for concomitant thermal (HHV basis) and carbon efficiencies. Similar data exist for the principal indirect routes - i.e., Fischer-Tropsch and methanol-to-gasoline. The former, an older technology, is based upon the gasification of coal to synthesis gas, i.e., $\mathrm{CO} / \mathrm{H}_{2}$ mixtures and their reactions on ruthenium/iron supported catalysts. The action of the traditional Fischer-Tropsch catalysts is to shunt hydrocarbon building blocks on to existing units and to allow two basic reaction paths, one to desorb a stable species and the second to attach an additional building block for subsequent reaction. In consequence, there is a geometric decrease in the probability of finding higher hydrocarbons in the resultant product; methane is the primary product which has to be considered either as a co-generated product, recycled to a steam reformer to produce additional syngas, or as a "waste" material to be used elsewhere in the process as a heating source. The latter is particularly undesirable since Fischer-Tropsch synthesis is exothermic, producing an excess heat source. In fact, Hoogendoorn (1977) indicates that SASOL II (the Fischer-Tropsch type process in commercial use in south Africa) would have a thermal efficiency of $35-38 \%$ if based on recycled methane, and of $60 \%$ if methane were sold as a gaseous product.

Schreiner et al. (1978) give a comparison of a Fischer-Tropsch process and a methanol-to-gasoline process. Considering the former for the moment, one may follow its various recycles and processes to determine the fate of the original coal carbon step by step. From these data, it is possible to construct bar graphs as shown in Figure $\mathrm{C}_{3}$ for the carbon and thermal efficiencies (HHV) basis for that process; again, separate bars are included for the liquid and gaseous portion of the product spectrum (here the gaseous spectrum is considered to be $C_{4}$ or lower). A similar analysis can be carried out for the coal-methanol-gasoline route with zeolite catalysis, and is also shown in Figure C3.

An evaluation can also be made of gasification processes. Pflasterer et al. (1980) discuss a high BTU plant using a "standard" Illinois 非 6 bituminous coal, in sufficient detail to produce the "High BTU gasification" entry in Fig. C3.

The data shown in $\mathrm{Figure} C 3$ are taken from the several quoted sources. The data shown in Figure C 4 were provided by Shih (1981) from additional sources. In particular, the bar graphs for H-Coal and SRC were derived from Fluor (1976) and SRI (1979). The efficiencies for these processes are considerably lower than those quoted in Figure C3. The reason is that the spectrum of products (see Table C2) contains more "light" products than that quoted by McNamee et al, 1978, for the corresponding generic SRC and catalytic hydroliquefaction processes studied by them.

We have previously asserted that the $\mathrm{CO}_{2}$ effluent from a synthetic fuel plant is flow sheet dependent; we have shown that the carbon efficiency and the thermal efficiency of a process are directly related. In Table C 3 we quote thermal efficiencies from a variety of sources for the same titular process. 


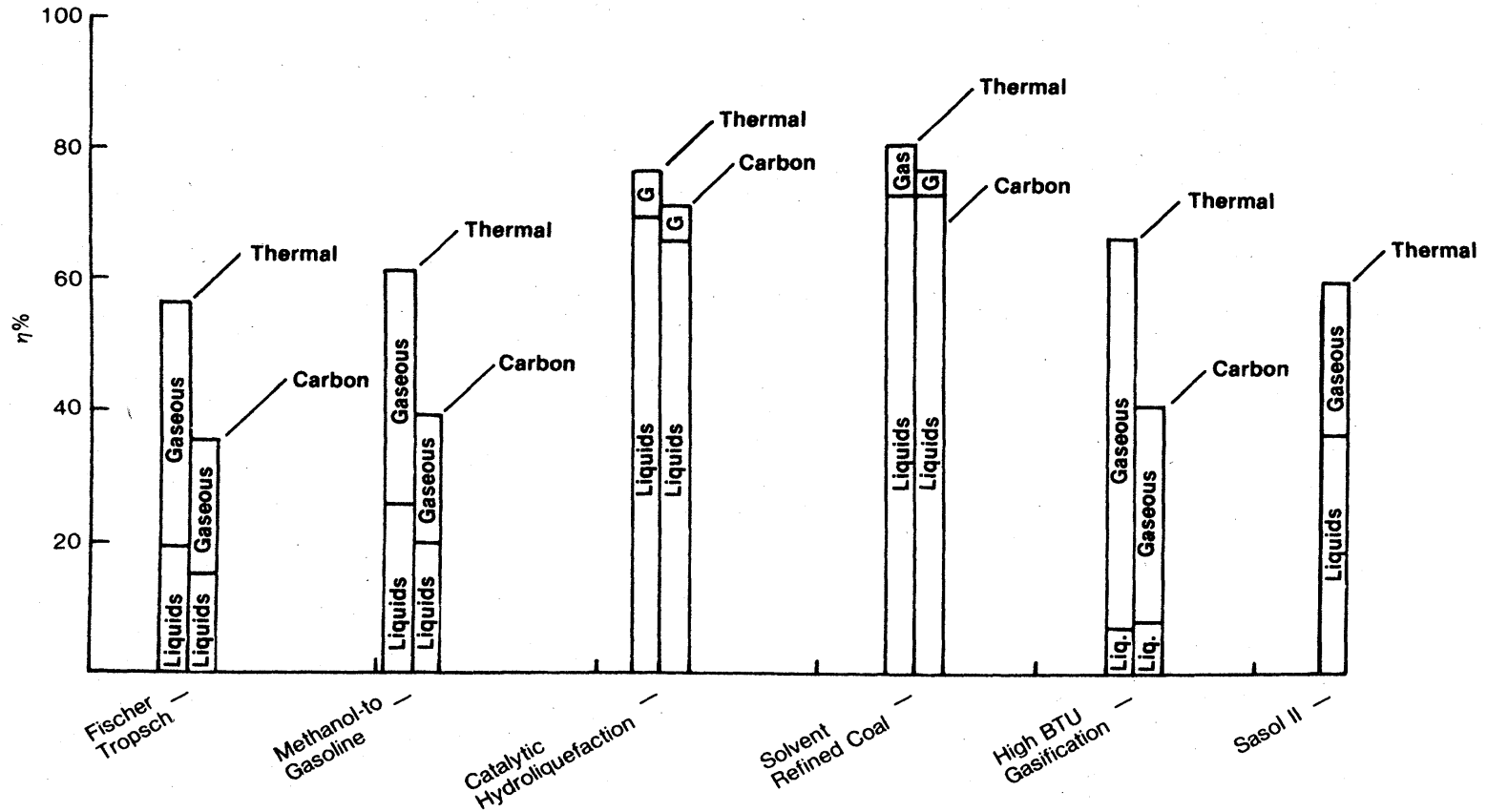

FIG. C3. Representative thermal and carbon efficiencies of several synthetic fuel routes.

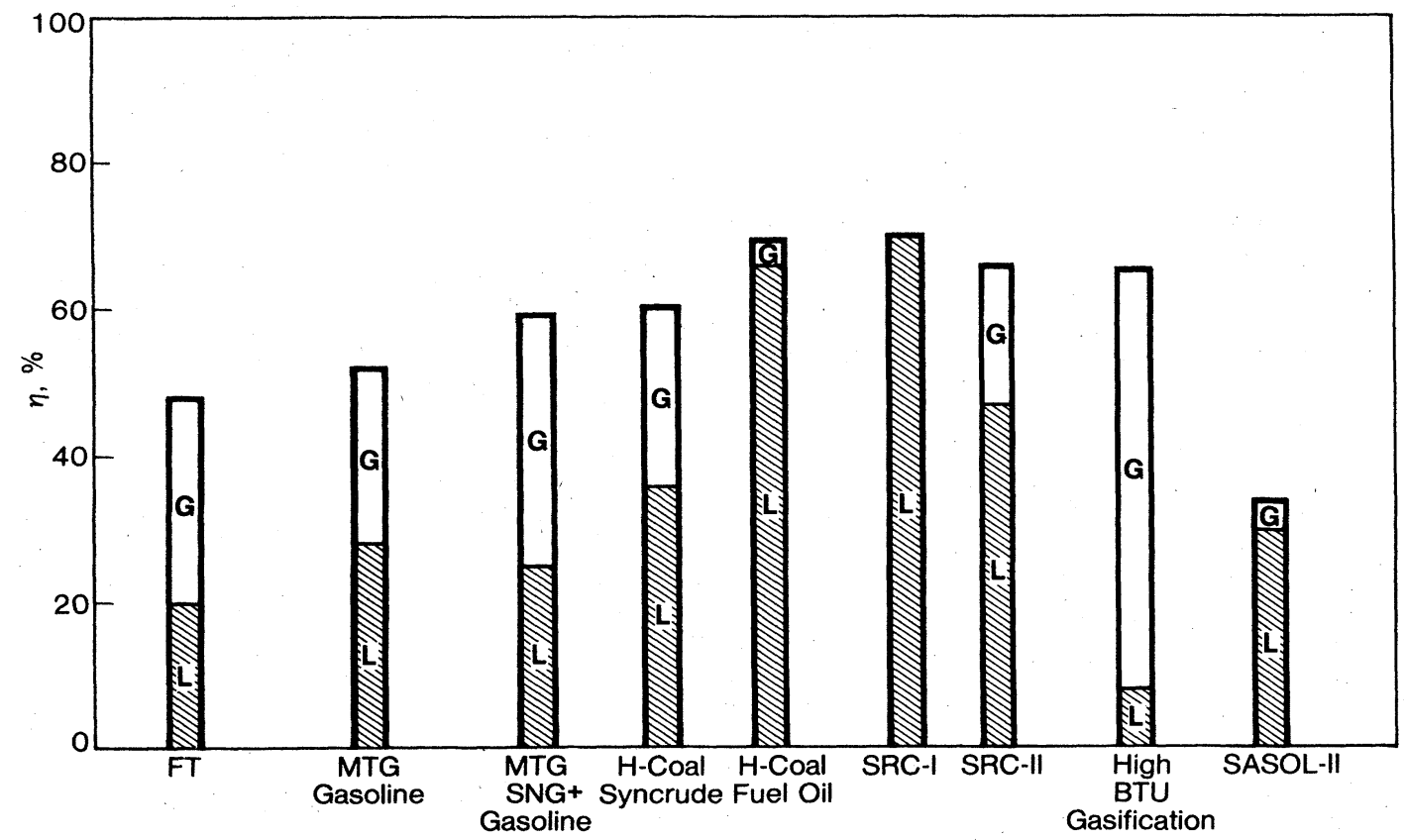

FIG. C4. Thermal efficiencies of some coal conversion processes (after Shih, 1981). 
Table C3: Quoted $n^{\mathrm{HHV}}$ for several named processes.

Process

$\mathrm{SRC}-\mathrm{I}$

SRC-II

SRC-I

SRC-II

Generic SRC

$\mathrm{H}-\mathrm{Coa1}$ (1)

$\mathrm{H}-\mathrm{Coal}$ (2)

$\mathrm{H}-\mathrm{Coal}$ (1)

$\mathrm{H}-\mathrm{Coal}$ (2)

Generic Catalytic Liquefaction

(1) Fuel Oil Mode

(2) Syn Crude Mode

\section{Thermal Efficiency}

70

70

71.5

65.8

82

74 (LHV)

69 (LHV)

59.2

60.0

76
Source

Harrison, 1980

Harrison, 1980

SRI, 1979

SRI, 1979

McNamee et al., 1978

Harrison, 1980

Harrison, 1980

Fluor, 1976

Fluor , 1976

McNamee et al., 1978
Significant differences emerge among them; the bar chart, Figure C3, must be viewed in the light of these differences.

The sources of $\mathrm{CO}_{2}$ from these processes primarily arise from the need for plant power, for steam to run the various auxiliaries and chemical purification steps required in a chemical plant, and for production of $\mathrm{H}_{2}$. All of these components of $n^{c}$ are significant. The hydrogen production step causes CO emission for two reasons: 1) Co shift to hydrogen; 2) Excess steam to effect this shift. That is to say, hydrogen is made from syngas by shifting according to $\mathrm{CO}+\mathrm{H}_{2} \mathrm{O} \rightarrow \mathrm{CO}_{2}$ $+\mathrm{H}_{2}$ in which 1 mole of $\mathrm{CO}_{2}$ is released per mole of $\mathrm{H}_{2}$ formed; but, in addition, excess steam is needed to drive the reaction to the right and this involves additional combustion of fuel.

It should now be evident that the thermal and carbon efficiencies of a large number of processes are directly related. There is roughly a linear relationship between these efficiencies. Insofar as improvements in thermodynamic efficiency are directly relatable to product costs, studies directed toward thermodynamic/economic goals will have the beneficial by-product of decreasing expected $\mathrm{CO}_{2}$ emissions from synthetic fuel plants, a point not specifically noted previously.

\section{Estimate of total $\mathrm{CO}_{2}$ releases}

For the purpose of eomparing $\mathrm{CO}_{2}$ emission from synthetic fuel production to that from coal powered electricity plants, we use the onetime DOE announced goal of $2.5 \times 10^{6}$ barrels/day of synthetic fuel. From the preceding discussion in this Appendix, we see that a typical value of the carbon efficiency of $\eta^{c} \approx 50 \%$ is reasonable. We then find that the rate of $\mathrm{CO}_{2}$ emission for this rate of synthetic fuel production at this carbon efficiency is the same as that from thirty 1000 megawatt coal-to-electricity plants having a thermal efficiency of $35 \%$ and being 
run at an energy output of $90 \%$ of their annual capability. The total effluent for both examples is 60 megatonnes of carbon or 220 megatonnes of $\mathrm{CO}_{2}$. For purpose of comparison, this amount of $\mathrm{CO}_{2}$ is about $0.008 \%$ of the 1975 atmospheric $\mathrm{CO}_{2}$, and equals $43 \%$ of the $\mathrm{CO}$ from flare gas burned off worldwide at refineries and oil fields in 1975. While this number of power plants produces the same $\mathrm{CO}_{2}$ as the quoted amount of synthetic fuel oif production with a carbon efficiency of $50 \%$, end-utilization of the synthetic fuel will cause an additional equal emission of $\mathrm{CO}_{2}$.

It is also possible to compare our estimates of $\mathrm{CO}_{2}$ evolution for different fuels, natural and synthetic, with those of MacDonald et al. (1980). These estimates, as shown in Table C4, refer to the total carbon released as $\mathrm{CO}_{2}$ in both processing and utilization.

The agreement between the two sets of estimates is good for the synthetic liquids processes ( $\mathrm{H}-\mathrm{Co}$ al and Catalytic Hydroliquefaction) and for coal to electricity. There is a significant difference between the MacDonald et al. estimates for HYGAS and ours for generic synthetic natural gas (SNG) production. This is due in the main to the (high and assumed) $50 \%$ carbon efficiency in the case of high BTU gasification (i.e. SNG entry for this report in Table (4) compared to the actual $n^{c}$ for the prototype HYGAS process. The higher ${ }^{c}$ corresponds to an increase in the thermodynamic efficiency that may be achievable by the introduction of one-step direct high BTU gasification processes such as a catalytic gasifier (Nahas and Gallagher, 1978) rather than using an inherently multi-step methanation process as in the HYGAS technology.

The most important conclusion of this section is the observation of the correlation between thermal and carbon efficiencies. The existence of this correlation should serve to reinforce the value of a research program directed toward the general improvement of synthetic fuel plant efficiency. The interested reader is again referred to previously quoted sources for global implications of this $\mathrm{CO}_{2}$ loading to the ecosystem.

\section{Use of non-fossil supplementary sources of process energ! for synthetic fuel production}

While it is clear that it should be possible to reduce $\mathrm{CO}_{2}$ production by use of non-fossil sources of process energy in

Table C4. Total (production \& utilization) carbon release rates compared to estimates of MacDonald et al, 1980

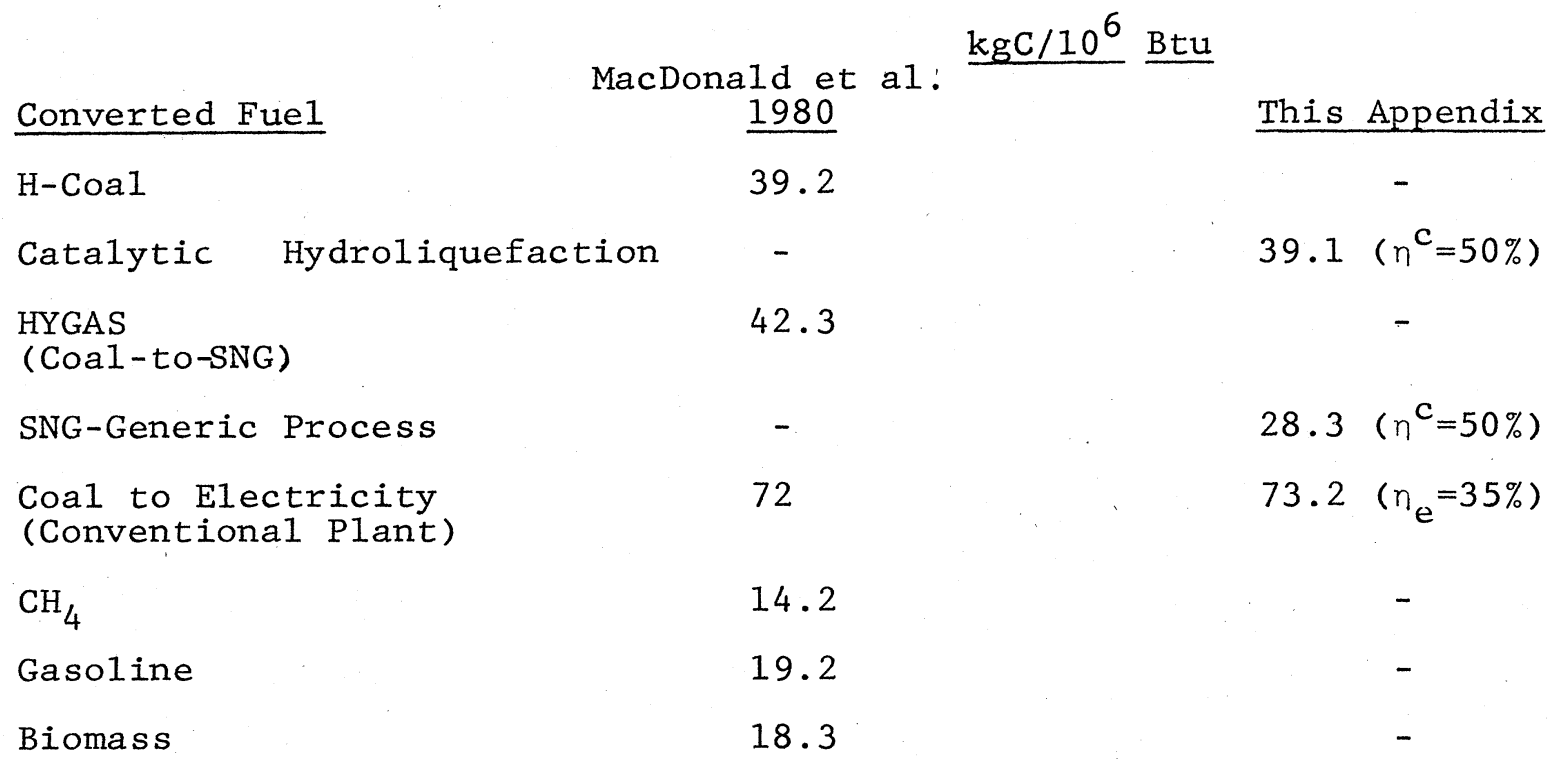


synthetic fuel production, the magnitude of this effect is not well known. Conceptually such alternative power supplies can be any non-fossil based source. For example, electrical energy input could be a substitute for a portion of the total process plant requirements. It might also pay to have high temperature process heat available for gasification and hydrogen production. The number of possible sources of process heat would be reduced to those capable of supplying heat at the pertinent conditions. Pflasterer et al. (1979) give data which indicate the requirements for high temperature $(>700 \mathrm{C})$ heat and for low temperature $(<300 \mathrm{C})$ heat (steam and electricity) are approximately equal in coal gasification. Lesser amounts of high temperature heat would be needed for coal liquefaction, and for supplying the hydrogen via gasification.

Irrespective of the source of alternative energy, one can easily estimate the reduction in effluent $\mathrm{CO}_{2}$ achievable by reducing the coal feed for a specified quantity and quality of product. Figure $\mathrm{C} 5$ shows the results of such a calculation. The ordinate is the $\mathrm{CO}_{2}$ effluent using a supplemental alternate (non-fossil) energy source compared to that

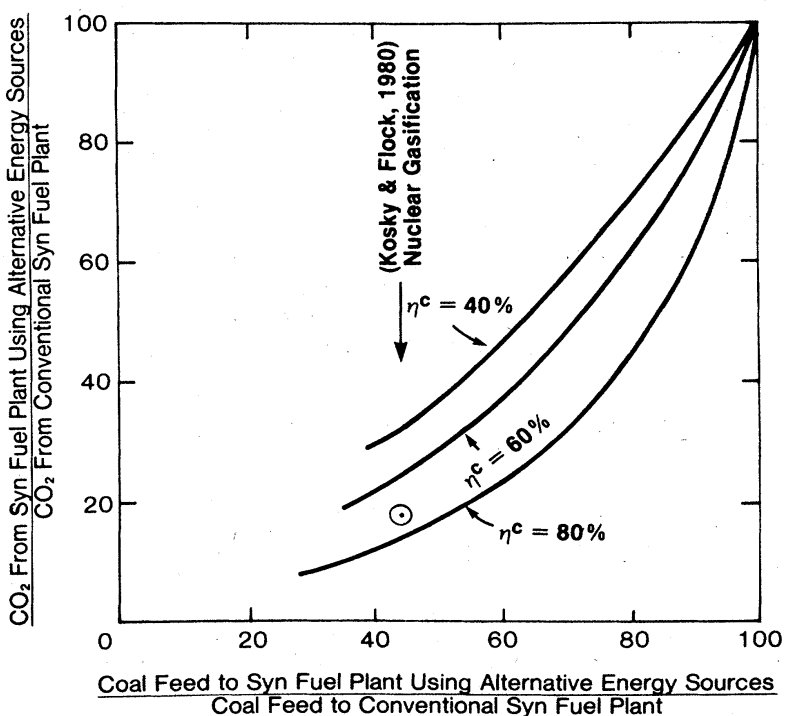

FIG. C5. Reduction of $\mathrm{CO}_{2}$ emission in synthetic fuel production by use of a supplementary non-fossil energy source (basis: same carbon in product). for a conventional plant with the same output. The reduction in $\mathrm{CO}_{2}$ is achieved by reducing the coal feed, here given as the abscissa in terms of the ratio of coal using alternate energy sources to that for the conventional plant of the same output.

For instance, Kosky and Flock (1980) have shown coal gasification efficiency can be increased by substitution of high temperature nuclear heat from a gas-cooled reactor in a conventional coal gasification process. In fact, the total coal consumption can be reduced by $44 \%$, of which $20 \%$ is in the gasifier proper, and $24 \%$ in process steam production. This has the effect of appreciably increasing the utilizable fossil carbon. This point is also plotted on Figure C5. Lesser reductions in coal feed are expectd for liquefaction plants (Pflasterer et al., 1979).

Economics dictate that the implementation of such coupling of technologies is most likely in countries in which fossil fuel prices are high, and conventional energy is in short supply. For example, the Federal Republic of Germany has an on-going aggressive program to match high temperature gas cooled reactors to coal gasifiers (von der Decken, 1975). However, Dietrich et al., (1975) have suggested that $\mathrm{CO}_{2}$ emission reduction would be a concomitant benefit to be derived, a point very recently reiterated by Haefele et al., (1980) in an IIASA study.

It is tempting to suggest that this is an area which may be of interest to the physics community. It is problematic as to whether the U.S. with its abundant coal supplies should consider this option. Furthermore, consideration of $\mathrm{Figure} \mathrm{C} 5$ indicates that $\mathrm{CO}_{2}$ can be reduced per unit of output in two ways -- by reducing the coal feed to a fossil plant using substitute non-fossil energy sources and/or by increasing the carbon efficiency at a fixed coal feed - the latter achievable by increasing the thermal efficiency of synthetic fuel plants.

\section{Conclusions}

Co release during synthetic fuel production is almost as ubiquitious as it is from utilization of fossil fuels. The amount of $\mathrm{CO}_{2}$ evolved in the synthesis process is inversely related to the thermal efficiency of the conversion process. For each process one 
may quote a range of valid thermal efficiencies based on product distribution and on the particular flow sheet chosen by the synthetic fuel plant designer. Conclusions as to the preferred synthetic fuel route based on $\mathrm{CO}_{2}$ evolution alone are probably premature at this time.

It appears that roughly as much $\mathrm{CO}_{2}$ will be produced during the manufacture of a synthetic fuel as will be produced during the use of that fuel. The amount of $\mathrm{CO}_{2}$ produced during synthesis is 220 megatonnes/year for 2 $1 / 2$ million barrels/day. This is equilvalent to about $30 \times 1000 \mathrm{MW}$ coal-fired electric generators or $43 \%$ of $\stackrel{e}{r}$ efinery and oil field gas flares estimated in 1975 on a worldwide basis.

$\mathrm{CO}_{2}$ evolution from synthetic fuel plants can be ${ }^{2}$ reduced by either increasing the thermal efficiency of the process or by the use of non-fossil energy supplements to the processes. However, even for an infeasible $100 \%$ efficient synthetic fuel process, the $\mathrm{CO}_{2}$ environmental impact question still revolves around the overall global use of fossil fuels, whether natural or synthetic; a determination of the extent to which such use leads to an increase in atmospheric $\mathrm{CO}_{2}$ concentration; and further determination of ${ }^{2}$ the environmental effects of such an increase. This vital question therefore falls outside the scope of this study.

\section{References: Appendix I}

A.A.A.S.,1980, "Workshop on Environmental and Societal Consequences of a Possible $\mathrm{CO}_{2}$-Induced Climate Change," Conf.; Annapolis, MD, 4/2 - 4/6, 1979, USDOE, CONF : 704143 , UC-11.

Abarbanel, H., et al, 1980, "JASON Study The Carbon Dioxide Problem: DOE Program and General Assessment" SRI Project 5793, Rept. JSR-80-06.

Brown, D., 1980, private communication.

Charney, J. G. et al, 1979, "Carbon Dioxide and Climate: A Scientific Assessment," National Academy Sciences Rept. to Climate Research Board (NRC).

Corman, J. C. and G. R. Fox, 1976, "Energy Conversion Alternatives Study-G.E. Phase II Final Report," I, (Executive Summary) NASA-CR 134949.

Council on Environmental Quality, 1981 ,
"Global Energy Futures and the Carbon Dioxide Problem", January, 1981.

Decken, C. B. von der, et al, 1975, "Special Issues; High Temperature Reactor for Process Heat Application," Nuc. Eng. Des. 34, \#1.

Dietrich, G., H. G. Eickhoff, F. Niehaus, and H. F. Niessen, 1975, "Motivation for and Possibilities for using Nuclear Process Heat," Nucl. Eng. Des., 34, 3.

Fluor Engineers and Constructors, 1976 , "H-Coal - Commercial Evaluation" DOE Rept. FE-2002-12.

Haefele, W., et al, 1980, "Energy Alternatives for a Finite World", International Institute for Applied Systems.

Harrison, J., 1980, National Coal Board, Stoke Orchard, Glous. England, Private Communication.

Hoogendoorn, J. C., 1977, "Gas from Coal for Synthesis of Hydrocarbons, Status of SASOL II," Panel discussion, 9 th Synthetic Pipeline Gas Symp., (11/1/77).

Kosky, P. G., and J. W. Flock, 1980, "Nuclear Process Heat -- Application to Coal Gasification" General Electric C. Reprt. 80CRDI51.

MacDonald, G., et al, 1980, "JASON Study The Long-Term Impacts of Increasing Atmospheric Carbon Dioxide Levels," U.S. DOE Contract, ME-AM-03-76SF-00115, SRI Project 5793, Rept. JSR-79-04.

MacDonald, G. et al, 1979, Ibid, U.S.D.O.E. Contract EY-76-C-0115, p. 136, SRI Project 5793, Rept. JSR-789-07.

McNamee, G. P., N. K. Patel, T. R. Roszkowski and G. A. White, 1978 , "Process Engineering Evaluation of Alternative Coal Liquefaction Concepts," EPRI AF-741, 1 .

Nahas, W. C., and S. E. Gallagher, Jr., 1978 , "Catalytic Gasification Predevelopment Research," 13th IECEC, 3 , p. 2143 (San Diego, CA).

Pflasterer, G. R., et al, 1979, "HTR-Synfuel Application Assessment," USDOE Rept. C0O-4057-12.

Pomeroy, B. D., J. Fleck, W. Marsh, D. Brown and R. Shah, 1978, "Comparative Study and Evaluation of Advanced Cycle Systems," EPRI-AF 664, 2, pt. 1 .

Schreiner, M. et al, 1978, "Research Guidance Studies to Assess Gasoline from Coal to Methanol-to-Gasoline and SASOL-Type Fischer-Tropsch Technology," USDOE Rept. FE2447-13. 
Shah, R., D. Ahner, G. Fox and M. Gluckman, 1980 ," Performance and Cost Characteristics of Combined Cycles Integrated with Second Generation Gasification Systems," ASME Gas Turbine Conference, Paper 80GT-106 (3/11/80).

Shih, S. S., 1981, private communication.

Shinnar, R., et al, 1978, "Gasifier Study for Mobil Coal to Gasoline Process," USDOE Rept. FE-2766-13.

Stanford Research Institute, 1979, Energy, Economics and Technology Program, 14. "Coal Liquefaction".

Wei, J, 1981, to be published in Ind. Eng. Chem., Proc. Des. Dev. 
Rev. Mod. Phys., Vol. 53, No. 4, Part II, October 1981 


\section{APPENDIX II. ACKNOWLEDGMENTS}

We wish to express our particular thanks to J. A. Burton, Treasurer of the American Physical Society, for his interest and aid in all aspects of this study. The administrative assistance of a number of other people at the American Physical Society is gratefully acknowledged.

The Study Panel benefited greatly from the encouragement and advice provided by the APS Review Committee chaired by H. Brooks. The membership of the Review Committee is listed at the beginning of this Report.

Support and comments from many of the personnel of the U.S. Department of Energy were valuable in initiating and carrying out the study. Many of these people are acknowledged below for supplying technical information to the Study Panel. However, we would like to particularly thank those people at DOE who worked with us in initiating the study and in maintaining contact during its course: J. R. Powers, C. D. W. Thornton, and J. A. Snow of the Office of Energy Research; R. Roberts and I. Wender of the Office of the Assistant Secretary for Fossil Energy. We would also like to thank the Director of the Office of Energy Research, E. A. Friemen and subsequently N. D. Pewitt, and the Assistant
Secretary for Fossil Energy, G. Fumich and subsequently R. W. A. LeGassie for the support of their Offices in the conduct of this study. We are grateful for the hospitality of West Virginia University which as provided facilities for the study headquarters. W. E. Vehse - Chairman of the Physics. Department and R. Koppelman - Vice-President for Energy Studies, Graduate Programs, and Research, have been helpful in many ways; and the West Virginia University Foundation has provided useful administrative services. Our special gratitude goes to the Study secretaries - to C. A. Flagg, who bore the brunt of the effort, and to K. J. Bukrim and J. A. Cooper.

The Study Panel and Review Committee benefited from the hospitality of a number of institutions at which our meetings were held. We are particularly thankful to B. C. Frazer and his colleagues at the American Physical Society editorial offices on Long Island for their hospitality and consideration during a very hectic two-week workshop in August, 1980. We also appreciate the courtesy extended to us during shorter meetings of the Study Panel hosted by Mobil Research and Development, the Lawrence Berkeley Laboratory of the University of California, the U.S. Department of Energy Germantown, MD, and the headquarters of the American Physical Society in New York City.

We are indebted to the following for meeting with and providing technical information to members of the Study Panel:
E. W. Albaugh

R. A. Alpher

L. G. Austin

W. Bakker

J. Beer

J. Birkeland

E. Burwe11

H. R. Child

R. Classen

S. J. Dapkunas

R. Datta

A. Davis

A. Dietz

S. Ergun

W. Farthing

R. Fischer

B. C. Frazer

J. Gethner

P. Given

D. W. Goodman

F. J. Grunthaner
Gulf Research \& Development Company

General Electric Research \& Development Center Pennsylvania State University

Electric Power Research Institute

Massachusetts Institute of Technology

U.S. Department of Energy - Germantown

U.S. Department of Energy - Germantown Oak Ridge National Laboratory

Sandia National Laboratories

U.S. Department of Energy - Germantown

Gulf Research \& Development Comapny

Pennsylvania State University

U.S. Department of Energy - Germantown

Lawrence Berkeley Laboratory

Southern Research Institute

U.S. Department of Energy - Germantown

Brookhaven National Laboratory

EXXON Research \& Engineering Company

Pennsylvania State University

Sandia National Laboratories

Jet Propulsion Laboratory 
D. R. Hardesty

R. Hildebrand

L. Iton

J. W. Larsen

T. K. Lau

B. Lewis

H. L. Love11

P. T. Luckie

R. Markley

D. W. McKee

T. P. Meloy

L. Miller

R. R. Oder

P. Painter

W. Peters

A. C. Raptis

H. L. Retcofsky

W. K. Rhim

K. W. Schatz

P. W. Schmidt

J. R. Schrieffer

P. Scott

S. M. Shapiro

P. G. Shewmon

B. G. Silbernage1

M. Sluyter

G. Somorjai

D. K. Stevens

F. D. Stevenson

J. Stringer

M. Strongin

J. A. Sullivan

F. Vastola

P. L. Walker, Jr.

R. T. Wood

R. A. Young
Sandia National Laboratories

U.S. Department of Energy - Germantown

Argonne National Laboratory

University of Tennessee

U.S. Department of Energy - Germantown

Jet Propulsion Laboratory

Pennsylvania State University

Pennsylvania State University

U.S. Department of Energy - Germantown

General Electric Research \& Development Center

West Virginia University

U.S. Department of Energy - Germantown

Gu1f Research \& Development Center

Pennsylvania State University

Pittsburgh Energy Technology Center - DOE

Argonne National Laboratory

Pittsburgh Energy Technology Center - DOE

Jet Propulsion Laboratory

Mobil Research \& Development Center

University of Missouri - Columbia

University of California - Santa Barbara

U. S. Department of Energy - Germantown

Brookhaven National Laboratory

Ohio State University

EXXON Research \& Engineering Company

U.S. Department of Energy - Germantown

University of California - Berkeley

U.S. Department of Energy - Germantown

U.S. Department of Energy - Germantown

Electric Power Research Institute

Brookhaven National Laboratory

Los Alamos National Laboratory

Pennsylvania State University

Pennsylvania State University

General Electric Research \& Development Center

University of Arizona

We are indebted to the following for critically reviewing drafts of this Report in part or in its entirety:

R. Alpher

R. Bacastow

W. A. Baum

R. S. Berry

W. Beezhold

H. R. Child

A. Damask

W. A. Ellingson

J. E. Epperson

M. Greenspan

D. R. Hardesty

H. Heinemann

L. Iton

K. Jordan
General Electric Research \& Development Center

University of California - San Diego

Florida State University

University of Chicago

Sandia National Laboratories

Oak Ridge National Laboratory

Queens College of the City University of New York

Argonne National Laboratory

Argonne National Laboratory

National Bureau of Standards

Sandia National Laboratories

Lawrence Berkeley Laboratory

Argonne National Laboratory

University of Pittsburgh 
J. J. Kaufman

L. Klemm

A. Levy

D. R. Lide, Jr.

P. Luckie

G. MacDonald

D. W. McKee

T. P. Meloy

P. A. Montano

W. A. Nierenberg

W. Peters

R. Perkins

E. H. Piepmeir

A. C. Raptis

H. L. Retcofsky

R. Rotty

J. Schooley

B. G. Silbernage1

P. L. Walker, Jr.

W. H. Weinberg

I. Wender

J. 0. L. Wendt

R. T. Wood

T. Yule
Johns Hopkins University

University of Oregon

Lawrence Berkeley Laboratory

National Bureau of Standards

Pennsylvania State University

MITRE Corporation

General Electric Research \& Development Center

West Virginia University

West Virginia University

University of California - San Diego

Pittsburgh Energy Technology Center - DOE

Lockheed Research Laboratories

Oregon State University

Argonne National Laboratory

Pittsburgh Energy Technology Center - DOE

Oak Ridge Associated Universities

National Bureau of Standards

EXXON Research and Engineering Company

Pennsylvania State University

California Institute of Technology

University of Pittsburgh

University of Arizona

General Electric Research \& Development Center

Argonne National Laboratory 
Rev. Mod. Phys., Vol. 53, No. 4, Part II, October 1981 


\section{APPENDIX III. GLOSSARY}

AES: Auger Electron Spectroscopy

ANTHRACITIC: the highest (most completely metamorphosed) ranks of coal with high fixed carbon percentages ( $86 \%$ or more) and low volatile content

A L I PHATIC: organic hydrocarbons characterized by straight-or branched-chain arrangement of the constituent carbon atoms -- composed of three subgroups: (1) paraffins (alkanes), (2) olefins (alkenes), and (3) acetylenes (alkynes)

AROMATIC: organic hydrocarbons incorporating the basic (ring) structure of benzene

ARUPS: angle resolved ultraviolet photoelectron spectroscopy

BAG-HOUSE: an emission control system of a pulverized coal fired boiler in which cooled stack gases (combustion products) are passed through fabric filters to remove particulates

BASE-LOADED: specifies an electric generating station operated continuously at full capacity in order to take advantage of its high efficiency and lower cost. Other more expensive generators are used for peak demand (turbines fired with kerosene for example).

BITUMINOUS: intermediate ranks of coal -with lower fixed carbon percentages than anthracite and considerable volatile matter ( $15 \%$ to more than 30\%)

BOTTOMS: heavy tar-like residual material (hydrocarbons, other high molecular weight compounds and carbon) remaining after purification of crudes or other distillates

BTU: "British thermal unit"; the heat necessary to raise the temperature of 1 pound of water at its maximum density by I F; $1 \mathrm{Btu}=252.0$ Calorie $=1054 \mathrm{~J}$

CAKING: the propensity of coal to form a solid mass when the (pulverized) material is heated in the absence of air (as in the standard volatile matter determination)

CARBON EFFICIENCY INDEX $\left(n^{C}\right)$ : the percentage of carbon in the products of a process relative to that in the input coal

CARBONYLS: inorganic carbon compounds containing $\mathrm{CO}$ groups

CARS : coherent anti-Stokes Raman spectroscopy

CAT-CRACKER: catalytic cracker (chemical reactor for breaking up large hydrocarbon molecules into smaller ones in the presence of a catalyst)

CHAR: the solid residue resulting from pyrolysis or other conversion reaction of coal - - it has high fixed carbon and low volatile content, and contains residual coal minerals

CO-GENERATION: utilization of rejected heat in a steam generation plant so that the plant generates both electric power and heat for industrial processing or space conditioning

COKE: a char of high porosity and good mechanical strength - - therefore important in steel making

COMBINED CYCLE: a scheme for the generation of electric power in which two generating systems are operated in cascade so that one (the "bottoming" cycle) uses as input the heat rejected by the other ("topping") cycle

COMMINUTION: breaking a solid up into a powder or pieces (it is most commonly done by grinding or crushing but, in the case of coal, can be accomplished chemically and perhaps even by shock waves.)

CYCLONE SEPARATOR: a centrifugal device for separating suspended solid (or liquid) particles from a gas by inducing a gas flow of high vorticity resulting in wall deposition and fall-out collection

DEVOLATILIZATION: an alternate term for the pyrolysis or destructive distillation of coal by heating in the absence of air

DIRECT LIQUEFACTION: a liquefaction process in which coal is dissolved or dispersed in a solvent, and reacts with hydrogen to produce liquids wi thout breakup of the coal structure to produce low molecular weight intermediates (such as $\mathrm{H}_{2}$, $\mathrm{CO}$, or $\mathrm{CH}_{3} \mathrm{OH}$ )

DONOR SOLVENT: an organic solvent (used in coal liquefaction) usually a coal-derived liquid, which is capable of being directly hydrogenated (usually in the presence of a catalyst) and is capable, in turn, of transferring hydrogen to chemically active sites in coal

EBULLATING BED: a multi-phase system in which gas and/or a liquid passes upwards through a bed of solids, thereby keeping 
the particles in motion

ENDOR: electron nuclear double resonance

ENTRAINED FLOW: a co-current flow of particulates in a continuous phase fluid

EPRI: Electric Power Research Institute

ESR/EPR: electron spin resonance/electron paramagnetic resonance

ETHERS: organic oxides -- compounds in which a single oxygen atom is linked to two separate organic groups

EXAFS: extended X-ray absorption fine structure

EXINITE: (See Liptinite)

EDS or EXXON DONOR SOLVENT: a coal liquefaction process in which the donor solvent (a coal-derived organic liquid) is catalytically hydrogenated in a separate reaction chamber before being brought into interaction with the coal

FBC: fluidized bed combustion (or combustor)

FINES: very finely pulverized coal produced incidental to grinding or crushing intended to produce a coarser product

FISCHER-TROPSCH: a commercial process for catalytic reactions of synthesis gas (hydrogen and carbon monoxide) into (mostly) straight and branched chain hydrocarbons

FLOTATION: various methods of "beneficiation" (separating, or purifying) of mixed pulverized solids (mineral ores or coal) which depend upon differences of density and/or wettability

FLUIDIZED BED: an arrangement in which crushed or pulverized solids in a bed are levitated because the gravitational forces are just balanced by hydrodynamic forces exerted by gas or liquid fed from below and flowing upward through the bed

FLUIDIZED FLOW: a mode of gas-solid and liquid-solid flow systems in which the solid particles are suspended in the flow field of the continuous phase, and exhibit fluid behavior

FREE RADICAL: a molecule with an odd number of electrons or, more generally, a chemical configuration containing more or less localized unpaired electrons

FRIABILITY: a tendency to break up or crumble under handling -- a quantitative index is defined by an ASTM test involving measurements of average sizes before and after one hour of being tumbled.

FTIR: Fourier transform infra-red spectroscopy

H-COAL: a coal liquefaction process (under development by Hydrocarbon Research, Inc.) in which coal, donor solvent, and catalyst are all mixed together in the primary reaction chamber under a hydrogen atmosphere, and the reactor is an ebullating bed

HEIS: high energy ion scattering

HETERO-ATOM: atom of an element other than carbon or hydrogen incorporated in the aromatic structure (as distinct from the mineral content) of coal -- most common are oxygen, sulfur, and nitrogen

HHV or HIGHER HEATING VALUE: heat of combustion of a hydrocarbon fuel assuming that fuel-bound hydrogen ends up as water in the liquid state

HREELS: high-resolution electron energy-loss spectroscopy

HYDROCRACKING: catalytic cracking (of large hydrocarbon molecules) in a hydrogen atmosphere to yield smaller molecules of higher hydrogen content

HYGAS: Institute of Gas Technology process for conversion of coal to substitute natural gas by hydrogasification, involving direct hydrogenation of coal in the presence of hydrogen and steam under pressure in sequential fluidized bed reactor stages

HYDROGEN DONOR SOLVENT: an organic solvent which is capable of transferring hydrogen to chemically active sites in coal

INDIRECT LIQUEFACTION: a liquefaction process which proceeds through the intermediate step of first making synthesis gas ( $\mathrm{H}$ and $\mathrm{CO}$ ), and subsequently synthesizes liquid hydrocarbons from this gas

INERTINITE: a group of macerals which is usually inert or semi-inert during normal carbonization or hydrogenation processes in a retort or reactor; they are opaque to transmitted light and bright in reflected light, and derived from charring of plant tissues or possibly as the result of intensive biochemical processes.

LEED: low energy electron diffraction

LEIS: low energy ion scattering

LHV OR LOWER HEATING VALUE: heat of combustion of hydrogen containing fuel assuming that fuel-bound hydrogen ends up as water in the vapor phase

LIGNITE: the lowest (least metamorphosed) rank of coal with low fixed carbon (less than 69\%) and low calorific value $(8,300$ Btu/lb or less) 
LIPTINITE (EXINITE): a maceral group having the highest hydrogen content, comparatively low index of refraction and reflectivity, and derived from resinous and waxy material of plants, including resins, cuticles, spore and pollen exines, and algal remains

LOCK HOPPER: a hopper for feeding dry materials through a pressure difference by input admission at one pressure (usually low) with output doors closed, and subsequently emptying after the input doors are closed and the pressure has been equalized with the output pressure (usually high)

LURGI: (trade name) a commercially available and commericalliy used batch coal gasifier of fixed bed configuration

MACERALS: the most elementary petrographically distinguishable (by microscopic examination) constituents of the organic part of coal (the organic analog of minerals)

MEIS: medium energy ion scattering

METC: Morgantown Energy Technology Center

METHANOL-TO-GASOLINE: a process developed by Mobil Research and Development Corp. in which a ZSM-5 catalyst is used to convert methanol ( $\mathrm{CH}_{3} \mathrm{OH}$ ) ( which can be synthesized through indirect coal liquefaction) into high octane gasoline

MHD: Magneto-hydrodynamic electrical power generation

MW : "megawatt electric" -- unit employed in specifying the capacity of an electric generating station

$\mathrm{MW}_{t}$ : "megawatt thermal" -- unit employed in specifying the heat input to an electric generating station

NATURAL GAS: high Btu fuel gas (about $1 \mathrm{x}$ $10^{6} \mathrm{BTU} / 1000 \mathrm{ft} .^{3}$ ) of natural origin -consisting mostly of methane $\left(\mathrm{CH}_{4}\right)$

NMR: nuclear magnetic resonance

NO : one (or a mixture) of the oxides of nitrogen

NQR: nuclear quadrupole resonance

PAS: photoacoustic spectroscopy

PCC: pulverized coal combustion (or combustor)

PESIS: photoelectron spectroscopy of the inner shells

PESOS: photoelectron spectroscopy of the outer shells

PETC: Pittsburgh Energy Technology Center

PETROGRAPHY: the study and differentiation of components of rocks and minerals by visual observation with a microscope
PHOTO-ACOUSTIC (MICROSCOPY, ETC.): detection and measurement of acoustic signals emitted as a consequence of optical irradiation or excitation of the specimen or system under study

PNEUMATIC CONVEYING: transport of pulverized solids which are entrained or suspended in a flowing gas and carried along with it

PROXIMATE ANALYSIS: characterization of coal in terms of moisture, ash content, fixed carbon, volatile matter, and calorific value

PYRIDINE: aromatic compound with hexagonal carbon ring having one substituted nitrogen atom $\left(\mathrm{C}_{5} \mathrm{H}_{5} \mathrm{~N}\right)$

PYRITES: 100 sely $\mathrm{y}^{5}$ sed to denote iron sulfides with a range of stoichiometry and crystal structures, an important mineral constituent and source of sulfur in coal

PYROLYSIS: decomposition by heating (in the absence of air)

RANK: a classification scheme for coals based primarily on their heating values (per unit weight) in direct combustion, and increasing with per cent carbon

REGENERATIVE HEATER: a heat exchanger in which the heated and cooled fluids occupy the same space by turns -- respectively receiving heat from and imparting heat to a storage medium

SAM: scanning Auger microscopy

SANS: small angle neutron scattering

SASOL: South African Synthetic Oil -- the world's oñly operating commercial plant for production of synthetic liquid fuels from coal (by the "indirect" Fischer-Tropsch process -- located in South Africa)

SAXS: small angle X-ray scattering

SCF : "standard cubic foot"; the amount of gas which occupies one cubic foot at normal atmospheric pressure and $60 \mathrm{~F}$

SCRUBBER: a sub-system of a pulverized coal fired boiler in which stack gases (combustion products) are treated with alkaline aqueous sprays to remove sulfur oxides

SEM: scanning electron microscopy (detects the secondary back scattered electrons)

SEXAFS: surface extended X-ray absorption fine structure

SIMS: secondary ion mass spectroscopy

SINGLE PARTICLE MONITOR: a device which detects individual particles of an aerosol or other aggregate and, from a 
statistically large number of such events, provides direct information about number concentration, and distribution in size or other physical properties, of the particles

SLAG: ash in the molten state -- usually a mixture (mostly) of oxides and complex silicates

SLUGGING: the formation of large particulate voids or bubbles in multiphase fluidized flow which grow to emcompass the entire channel perpendicular to the flow

SNG: "substitute natural gas" - manufactured or man-made high Btu fuel gas consisting mostly of methane $\left(\mathrm{CH}_{4}\right)$

SO : one (or a mixture) of the oxides of sulfur

SRC I and SRC II: "solvent refined coal", I and II, -- processes for cleaning up and hydrogenating coal using a coal-derived solvent and hydrogen atmosphere, but without added catalyst. SRC-I produces pitch-like solid fuels; SRC-II produces liquid fuels.

"STIFF": (of differential equations) equations expressing the coupling or interaction of systems or processes having very disparate time-constants

SUPERHEATER: the highest temperature stage or tubing bank of a steam boiler

SYNGAS (SYNTHESIS GAS): the mixture of hydrogen and carbon monoxide produced by the carbon steam reaction (and its concommitants) in a coal gasifier

TDR: time domain reflectometry

TEM: transmission electron microscopy

THIXOTROPIC SLURRIES: gels (pseudofluids containing suspended solids) for which the shear stress is not linearly dependent upon the velocity gradient

TYPE: a classification scheme for coals according to maceral content

ULTIMATE ANALYSIS: an analytical scheme that is more complete than proximate analysis, including a detailed chemical content stated in terms of elemental constituents -- $\%$ C, $\% \mathrm{H}, \% \mathrm{O}$, etc.

ULTRAFINE PARTICLES: coal particles of average size $<1 \mu \mathrm{m}$

UPS: ultraviolet photoelectron spectroscopy

VITRINITE: the most abundant and important groups of macerals in coals, consisting of coalified woody tissues derived from stems, roots and vascular tissues of leaves

XPS: X-ray photoelectron spectroscopy

ZEOLITES: a family of alumino-silicates whose (unusually open) crystal structure is characterized by cages and channels of well defined shapes, giving high internal surface area - - sometimes called "molecular sieves" 